\title{
TWISTED GEOMETRIC SATAKE EQUIVALENCE VIA GERBES ON THE FACTORIZABLE GRASSMANNIAN
}

\author{
RYAN COHEN REICH
}

Abstract. The geometric Satake equivalence of Ginzburg and MirkovićVilonen, for a complex reductive group $G$, is a realization of the tensor category of representations of its Langlands dual group ${ }^{L} G$ as a category of "spherical" perverse sheaves on the affine grassmannian $\operatorname{Gr}_{G}=G(\mathbb{C}((t)) / G(\mathbb{C}[t]])$. Since its original statement it has been generalized in two directions: first, by Gaitsgory, to the Beilinson-Drinfeld or factorizable grassmannian, which for a smooth complex curve $X$ is a collection of spaces over the powers $X^{n}$ whose general fiber is isomorphic to $\mathrm{Gr}_{G}^{n}$ but with the factors "fusing" as they approach points with equal coordinates, allowing a more natural description of the structures and properties even of the Mirković-Vilonen equivalence. The second generalization, due recently to Finkelberg-Lysenko, considers perverse sheaves twisted in a suitable sense by a root of unity, and obtains the category of representations of a group other than the Langlands dual. This latter result can be considered as part of "Langlands duality for quantum groups".

In this work we obtain a result simultaneously generalizing all of the above. We consider the general notion of twisting by a gerbe and define the natural class of "factorizable" gerbes by which one can twist in the context of the Satake equivalence. These gerbes are almost entirely described by the quadratic forms on the weight lattice of $G$. We show that a suitable formalism exists such that the methods of Mirković-Vilonen can be applied directly in this general context virtually without change and obtain a Satake equivalence for twisted perverse sheaves. In addition, we present new proofs of the properties of their structure as an abelian tensor category.

\section{Contents}

\section{Part I. Prelude on gerbes}

I.1. Categorification of cohomology

I.2. Twisting categories by a gerbe

I.3. Equivariance and multiplicativity

I.4. Twisting structures by a gerbe

I.5. Construction of gerbes from algebraic geometry

\section{Part II. Symmetric factorizable gerbes and quadratic forms}

II.1. The affine grassmannian and factorizability

II.2. Gerbes and factorizability

II.3. Classification: the torus case

II.4. Multiplicative factorizable gerbes

II.5. The affine grassmannian of any group

Received by the editors October 17, 2010 and, in revised form, December 28, 2010; October 9, 2011; March 26, 2012; March 30, 2012.

2010 Mathematics Subject Classification. Primary $22 \mathrm{E} 57$. 
II.6. The determinant line bundle and its factorizable gerbe 385

II.7. Classification: the general case 388

Part III. Equivariance of symmetric factorizable gerbes

III.1. Infinitesimal actions on the grassmannian 394

III.2. Factorizable gerbes are naturally equivariant 402

\section{Part IV. Relative twisted geometric Satake equivalence 406}

IV.1. Orbits in the affine grassmannian $\quad 406$

IV.2. Vanishing cycles and gluing $\quad 410$

IV.3. Convolutions $\quad 418$

IV.4. The fiber functor 423

IV.5. The main theorem for a torus 427

IV.6. Absolute twisted Satake: semisimplicity 429

IV.7. Absolute twisted Satake: root data 434

IV.8. Relative twisted Satake 442

Part V. Connections 443

V.1. Relation to the result of Finkelberg-Lysenko 444

V.2. Relation to Lusztig's quantum groups

V.3. Alteration of the commutativity constraint 447

V.4. Relation to the quantum Langlands correspondence 447

References 4449

\section{INTRODUCTION}

The main result (Theorem IV.8.3) of this paper is a synthesis of the geometric Satake equivalence, as proved by Mirković and Vilonen in MV07, the twisted Satake equivalence, as proved in Finkelberg and Lysenko [FL10, and Gaitsgory's factorizable Satake equivalence [Gai07, Theorem 2.6]. A historical overview of the Satake equivalence up until its proof in the first citation can be found there; here, we will give a brief description of how this paper's techniques are understood by the author and how and why they differ from those of the latter two citations.

Let $G$ be a connected, reductive algebraic group over $\mathbb{C}$; the restriction to the complex field is dictated by the central role played by its root data, namely, the quadruple $\left(X^{*}, X_{*}, \Psi^{*}, \Psi_{*}\right)$ consisting of the weights (characters) and coweights of some maximal torus of $G$ and the roots and coroots contained in these lattices. The basic Satake equivalence describes the category of representations of the Langlands dual group ${ }^{L} G$ whose root data is $\left(X_{*}, X^{*}, \Psi_{*}, \Psi^{*}\right)$; the twisted Satake equivalence generalizes this duality operation by including the data of a certain integer $q$ (the manner of which is described in Section V.1 and arriving at a "dual" group $\breve{G}_{q}$. In both cases, the description is as a certain tensor category of perverse sheaves on the affine grassmannian $\mathrm{Gr}_{G}$; the details of this correspondence can of course be found in Part IV As for $G$, we define $\mathrm{Gr}_{G}$ over $\mathbb{C}$ and will use topological concepts from the classical topology. We assume some familiarity with the affine grassmannian and the proof of the Satake equivalence in this introduction. 
Over time, the Satake equivalence has come to be seen less as a theorem about representation theory as it occurs in certain cohomology groups and more as a geometric theorem about the structure of the affine grassmannian. The single most important event in that shift was probably Mirkovic and Vilonen's use of the factorizable grassmannian to state and prove the fusion product formula (Proposition IV.3.4) (1) . The factorizable grassmannian (Definition II.1.1) was elevated by Gaitsgory to the fundamental setting of the theorem and in this paper we use it exclusively, except for the specific technical computations of Section IV.7 (and the supporting preceding section).

We introduce two main technical tools in this paper. By far the more prevalent is that of symmetric factorizable ( $s f$ ) gerbes (Definition II.2.1), which replace the twisting integer $q$ of Finkelberg and Lysenko. There is a simple explanation for why gerbes should appear at all: the manner of twisting in that paper is by considering sheaves on a $\mathbf{G}_{\mathbf{m}}$-bundle $\operatorname{det}_{G}$ over $\mathrm{Gr}_{G}$ that satisfy a form of "twisted decent": their restrictions to each fiber are not constant but are local systems (representations of $\pi_{1}\left(\mathbf{G}_{\mathbf{m}}\right) \cong \mathbb{Z}$ ) of monodromy $q$. If $\operatorname{det}_{G}$ is trivialized on an open set $U \subset \operatorname{Gr}_{G}$, such sheaves are unnaturally identifiable with sheaves on $U$, but such data on $\operatorname{Gr}_{G}$ does not glue. Instead, it can be seen (over a sufficiently fine covering) to constitute a Cech 2 -cocycle with values in $\mathbb{C}^{*}$, and this concept of twisted gluing corresponds to twisting by a gerbe (Section I.2).

The advantage of using gerbes on the grassmannian itself to twist is that there is a clear extension of the formalisms of sheaves to gerbe-twisted sheaves. It also clarifies the aspects of the proof of the Satake equivalence that are "natural" and those that are "noncanonical"; for example, global cohomology is noncanonical from the gerbe perspective. This precipitates a complete adoption of the geometric point of view and, in fact, there are no cohomology computations anywhere in this paper. The ultimate validation of this point of view is in Theorems II.7.3 and III.2.10, which show that gerbes can be classified and given the essential structure of $G(\widehat{\mathcal{O}})$-equivariance (see Definition III.1.1) purely on the basis of the geometric property of factorizability. We find it significant that these theorems closely mirror the Satake equivalence for sheaves; together, they appear to constitute a Satake equivalence for gerbes.

Our other technical tool is the use of ULA perverse sheaves (Definition IV.2.6) through the essential Lemma IV.2.14, which is based on the very useful nearbycycles gluing theorem of Beilinson. Through it we are able to give short and conceptual proofs of all the main ingredients of the Satake equivalence: convolution product (Proposition IV.3.4), commutativity constraint (via the fusion product), compatibility of the fiber functor (Proposition IV.4.2), and also the generalization from the "absolute" (on $\mathrm{Gr}_{G}$ ) theorem (Theorem IV.7.15) to the "relative" (factorizable) theorem (Theorem IV.8.3). We are also pleased to have been able to demonstrate the semisimplicity theorem on $\mathrm{Gr}_{G}$ (Proposition IV.6.13) without the use of [Lus83, §11], which was previously the only known method.

The present article was prepared on the basis of my thesis, for which I give my deepest gratitude to my advisor, Dennis Gaitsgory. His insights into the use of nearby cycles (literally and figuratively) led to the two fundamental technical results of this work: Lemma I.5.1 and Lemma IV.2.14, as well as numerous other essential contributions. 


\section{Part I. Prelude on gerbes}

As we use gerbes in an essential and sometimes elaborate way throughout this paper, we will need to introduce their basic properties in Part I before moving on.

\section{I.1. Categorification of Cohomology}

Let $X$ be a topological space; except for Section I.5, it could also be an arbitrary site. Let $\mathcal{G}$ be a sheaf of abelian groups on $X$, and fix the notation

$$
\mathscr{H}^{1}(X, \mathcal{G})=X / \mathcal{G}
$$

for the fibered category with descent (i.e. "stack", or "sheaf of categories") of $\mathcal{G}$ torsors on $X$; for a detailed description of basic facts about stacks, we recommend the chapter by Vistoli in $\mathrm{FGI}^{+} 05$. Since $\mathcal{G}$ is abelian, this stack is in fact an abelian group stack, or "Picard stack". We assume that a stack comes with a splitting (choice of pullbacks); since all our fibered categories will be of sheaftheoretic origin, this will always be true.

Definition I.1.1. Let $\mathscr{G}$ be a stack with an action of the stack $\mathscr{H}^{1}(X, \mathcal{G})$. We say that this action is a gerbe for $\mathcal{G}$ if for some (in fact, every) cover of $X$ by open sets $U$ in which each $U$ admits a section $s \in \mathscr{G}_{U}$, the map of stacks

$$
\left.\mathscr{H}^{1}(U, \mathcal{G}) \stackrel{\mathcal{T} \mapsto \mathcal{T} \cdot s}{\longrightarrow} \mathscr{G}\right|_{U}
$$

is an equivalence. We will denote by $\mathbf{H}^{2}(X, \mathcal{G})$ the 2 -category of gerbes on $X$, in which morphisms are $\mathcal{G}$-equivariant maps of stacks. We call the trivial gerbe $\mathscr{G}^{0}$ the regular action of $\mathscr{H}^{1}(X, \mathcal{G})$ on itself.

An even more general theory of gerbes is given in Giraud's thesis Gir71, but we will sketch the relevant aspects in this setting. Actual consultation of this (much more complete) work is not necessary to understand the present one, and its focus on non-abelian cohomology is actually somewhat orthogonal to the ends to which we put our gerbes.

The following proposition is immediate from the definition.

Proposition I.1.2. Any map of gerbes is an equivalence.

Proposition I.1.3. There is a tensor product of gerbes $\left(\mathscr{G}_{1}, \mathscr{G}_{2}\right) \mapsto \mathscr{G}_{2} \otimes \mathscr{G}_{2}$ which is associative, commutative, unital, and admits inverses all up to natural equivalence.

Proof. We define $\mathscr{H}_{\mathrm{om}_{\mathcal{G}}}\left(\mathscr{G}_{1}, \mathscr{G}_{2}\right)$ to be the stack whose sections on a neighborhood $U$ are the category of $\mathscr{H}^{1}(X, \mathcal{G})$-equivariant (and by definition, cartesian) functors from $\mathscr{G}_{1}$ to $\mathscr{G}_{2}$, and whose morphisms are natural transformations of such functors. It admits an $\mathscr{H}^{1}(X, \mathcal{G})$-action on the second variable (equivalently, the inverse action on the first variable), which is easily seen to be trivialized wherever both of the $\mathscr{G}_{i}$ are. We set

$$
\mathscr{G}^{-1}=\mathscr{H} \mathrm{om}_{\mathcal{G}}\left(\mathscr{G}, \mathscr{G}^{0}\right), \quad \mathscr{G}_{1} \otimes \mathscr{G}_{2}=\mathscr{H}_{\mathrm{om}}\left(\mathscr{G}_{1}^{-1}, \mathscr{G}_{2}\right) .
$$

One checks that $\mathscr{G}^{-1}$ has the same trivializations as $\mathscr{G}$ but with the inverse gluing data, and thus that $\left(\mathscr{G}^{-1}\right)^{-1} \cong \mathscr{G}$. This immediately gives $\mathscr{G}^{0}$ as both a left and a right identity. For inverses, both $\mathscr{G}^{-1} \otimes \mathscr{G}=\mathscr{H}_{\mathrm{om}_{\mathcal{G}}}(\mathscr{G}, \mathscr{G})$ and $\mathscr{G} \otimes \mathscr{G}^{-1}=$ $\mathscr{H} \mathrm{om}_{\mathcal{G}}\left(\mathscr{G}^{-1}, \mathscr{G}^{-1}\right)$ are globally trivial. More generally, from this interpretation of the inverse we see that $\mathscr{H} \mathrm{om}\left(\mathscr{G}_{1}, \mathscr{G}_{2}\right)=\mathscr{H} \mathrm{om}\left(\mathscr{G}_{1}^{-1}, \mathscr{G}_{2}^{-1}\right)$, so that inversion is an involution which is both a homomorphism and (by definition) an anti-homomorphism, 
proving commutativity of $\otimes$. To prove associativity one argues directly, which is straightforward if tedious.

This proof is valid without modification for the definition of the tensor product of two torsors as well, which gives the Picard category structure on sections of $\mathscr{H}^{1}(X, \mathcal{G})$.

Suppose we have a map of sheaves of groups $\phi: \mathcal{G} \rightarrow \mathcal{H}$, inducing a change-ofgroup functor which we denote ${ }^{1} \phi: \mathscr{H}^{1}(X, \mathcal{G}) \rightarrow \mathscr{H}^{1}(X, \mathcal{H})$. A similar 2-functor exists for gerbes:

$$
{ }^{2} \phi: \mathbf{H}^{2}(X, \mathcal{G}) \rightarrow \mathbf{H}^{2}(X, \mathcal{H})
$$

where denoting $\mathscr{H}_{\phi}^{0}=\mathscr{H}^{1}(X, \mathcal{H})$ with the action of $\mathscr{H}^{1}(X, \mathcal{G})$ given by ${ }^{1} \phi$, we have

$$
{ }^{2} \phi(\mathscr{G})=\mathscr{H}_{\mathrm{om}_{\mathcal{G}}}\left(\mathscr{G}^{-1}, \mathscr{H}_{\phi}^{0}\right)=\mathscr{G} \otimes \mathscr{H}_{\phi}^{0} .
$$

Here, $\mathscr{H}^{1}(X, \mathcal{H})$ acts on this via the second factor; since $\mathscr{G}$ is locally trivial, ${ }^{2} \phi(\mathscr{G})$ is locally $\mathscr{H}_{\mathrm{om}_{\mathcal{G}}}\left(\mathscr{G}^{0}, \mathscr{H}^{0}\right) \cong \mathscr{H}^{0}$, so is also locally trivial, hence an $\mathcal{H}$-gerbe. As for $\otimes$, this can be taken as an inductive definition of ${ }^{1} \phi$ as well. Since $\mathscr{H}^{0}$ is the unit in $\mathbf{H}^{2}(X, \mathcal{H})$ (so idempotent), we see by associativity of $\otimes$ that ${ }^{2} \phi$ is a tensor homomorphism.

Lemma I.1.4. For a $\mathcal{G}$-gerbe $\mathscr{G}$, we have ${ }^{2} \phi(\mathscr{G}) \cong \mathscr{H}^{0}$ if and only if there exists a $\mathscr{G}$-equivariant map $\mathscr{G} \rightarrow \mathscr{H}^{1}(X, \mathcal{H})$.

Proof. This is literally the criterion for ${ }^{2} \phi\left(\mathscr{G}^{-1}\right)$ to have a global section, hence be trivial, and this occurs if and only if the inverse gerbe ${ }^{2} \phi(\mathscr{G})$ is trivial.

The tensor product has a more natural description in terms of the group product:

Lemma I.1.5. The following equivalences hold:

(1) $\mathbf{H}^{2}(X, \mathcal{G} \times \mathcal{H}) \cong \mathbf{H}^{2}(X, \mathcal{G}) \times \mathbf{H}^{2}(X, \mathcal{H})$.

(2) Writing $m: \mathcal{G} \times \mathcal{G} \rightarrow \mathcal{G}$ for the product, we have $\mathscr{G}_{1} \otimes \mathscr{G}_{2}={ }^{2} m\left(\mathscr{G}_{1} \times \mathscr{G}_{2}\right)$.

Proof. For (1) Let $\mathrm{pr}_{1}, \mathrm{pr}_{2}: \mathcal{G} \times \mathcal{H} \rightarrow \mathcal{G}, \mathcal{H}$ respectively be the projections. We claim that for gerbes $\mathscr{G}, \mathscr{H}$ on the right, $\mathscr{G} \times \mathscr{H}$ is a gerbe for $\mathcal{G} \times \mathcal{H}$. Assuming this for the moment, both directions of the equivalence and their two composition isomorphisms are

$$
\begin{aligned}
& \mathscr{P} \mapsto\left({ }^{2} \operatorname{pr}_{1} \mathscr{P},{ }^{2} \operatorname{pr}_{2} \mathscr{P}\right), \quad(\mathscr{G}, \mathscr{H}) \mapsto \mathscr{G} \times \mathscr{H}, \\
& \mathscr{P} \rightarrow{ }^{2} \operatorname{pr}_{1} \mathscr{P} \times{ }^{2} \mathrm{pr}_{2} \mathscr{P}, \quad \mathscr{G} \rightarrow{ }^{2} \operatorname{pr}_{1}(\mathscr{G} \times \mathscr{H}) \text {, etc. }
\end{aligned}
$$

where each component of the one on the left comes from the trivialization in $\mathscr{H} \mathrm{om}\left(\mathscr{P} \otimes \mathscr{P}^{-1}, \mathcal{G}^{0}\right)$ and the one on the right comes from the same for $\mathscr{G}$ after forgetting $\mathscr{H}$.

To prove the deferred claim, apply the preceding construction to torsors rather than gerbes and then establish the claim directly; this shows that the lemma holds for $\mathscr{H}^{1}(X, \mathcal{G} \times \mathcal{H})$. Now replace $\mathcal{G}$ and $\mathcal{H}$ by $\mathscr{H}^{1}(X, \mathcal{G})$ and $\mathscr{H}^{1}(X, \mathcal{H})$ and repeat the argument to prove the claim for gerbes.

For (2). We have

$$
{ }^{2} m\left(\mathscr{G}_{1} \times \mathscr{G}_{2}\right)=\mathscr{H} \mathrm{om}\left(\mathscr{G}_{1}^{-1} \times \mathscr{G}_{2}^{-1}, \mathscr{G}_{m}^{0}\right)
$$

and we identify this with $\mathscr{H} \mathrm{om}\left(\mathscr{G}_{1}^{-1}, \mathscr{G}_{2}\right)$ by defining a map which, for any homomorphism $\phi$ in (I.2) defines a map $\psi: \mathscr{G}_{1}^{-1} \rightarrow \mathscr{G}_{2}$ sending a section $s$ of $\mathscr{G}_{1}$ to the unique section $t$ of $\mathscr{G}_{2}$ such that $\phi(s, t)=\mathcal{T}^{0}$ is the trivial torsor in $\mathscr{G}^{0}=\mathscr{H}^{1}(X, \mathcal{G})$. 
One checks that this is $\mathscr{H}^{1}(X, \mathcal{G})$-anti-equivariant in $s$ and equivariant in $t$ and so gives an isomorphism of gerbes.

The change-of groups functors are homomorphic in any way that makes sense:

Proposition I.1.6. We have ${ }^{2}(\psi \circ \phi)={ }^{2} \phi \circ{ }^{2} \psi$ for composable maps $\phi: \mathcal{K} \rightarrow \mathcal{G}$ and $\psi: \mathcal{G} \rightarrow \mathcal{Q}$, and ${ }^{2}(\phi \cdot \psi)={ }^{2} \phi \otimes{ }^{2} \psi$ for maps $\phi, \psi: \mathcal{G} \rightarrow \mathcal{H}$.

Proof. The first is another variant of associativity of $\mathscr{H}$ om and the second is a consequence of $\phi \cdot \psi=m \circ(\phi, \psi)$, where $(\phi, \psi): \mathcal{G} \rightarrow \mathcal{H} \times \mathcal{H}$, the previous lemma, and the first claim of this proposition.

There is a categorified long exact sequence of cohomology up to degree 2. Here, a sequence $\mathbf{A} \rightarrow \mathbf{B} \rightarrow \mathbf{C}$ of 2-Picard categories is exact if the induced sequence on equivalence classes of objects is exact and the induced sequence $\operatorname{Aut}\left(1_{\mathbf{A}}\right) \rightarrow$ $\operatorname{Aut}\left(1_{\mathbf{B}}\right) \rightarrow \operatorname{Aut}\left(1_{\mathbf{C}}\right)$ is an exact sequence of Picard categories (which is defined inductively in the same way).

Proposition I.1.7. If $1 \rightarrow \mathcal{K} \rightarrow \mathcal{G} \rightarrow \mathcal{Q} \rightarrow 1$ is a short exact sequence of sheaves of abelian groups, then we have a long exact sequence of groups/Picard categories/2Picard categories, where $\mathbf{H}^{1}(X, \mathcal{G})$ is the category of $\mathcal{G}$-torsors on $X$ :

$$
\begin{aligned}
& 1 \rightarrow \mathcal{K}(X) \stackrel{\phi}{\rightarrow} \mathcal{G}(X) \stackrel{\psi}{\rightarrow} \mathcal{Q}(X) \\
& \stackrel{\delta^{1}}{\longrightarrow} \mathbf{H}^{1}(X, \mathcal{K}) \stackrel{{ }^{1} \phi}{\longrightarrow} \mathbf{H}^{1}(X, \mathcal{G}) \stackrel{{ }^{1} \psi}{\longrightarrow} \mathbf{H}^{1}(X, \mathcal{Q}) \\
& \quad \stackrel{\delta^{2}}{\longrightarrow} \mathbf{H}^{2}(X, \mathcal{K}) \stackrel{{ }^{2} \phi}{\longrightarrow} \mathbf{H}^{2}(X, \mathcal{G}) \stackrel{{ }^{2} \psi}{\longrightarrow} \mathbf{H}^{2}(X, \mathcal{Q}) .
\end{aligned}
$$

Proof. Since $\psi \circ \phi=1$, we also have ${ }^{1} \phi \circ{ }^{1} \psi=1$ and ${ }^{2} \phi \circ{ }^{2} \psi=1$.

We define $\delta^{2}(\mathcal{T})$ for a $\mathcal{Q}$-torsor $\mathcal{T}$ to be the sheaf of categories whose category of sections over a neighborhood $U$ has objects the set of pairs $(\widetilde{\mathcal{T}}, f)$ where $\widetilde{\mathcal{T}}$ is a $\mathcal{G}$-torsor and $f$ is an isomorphism of ${ }^{1} \psi(\widetilde{\mathcal{T}})$ with $\mathcal{T}$; its morphisms are maps of the $\widetilde{\mathcal{T}}$-term inducing a commutative triangle after application of ${ }^{2} \psi$. Evidently, $\mathscr{H}^{1}(X, \mathcal{K})$ acts on $\delta^{2}(\mathcal{T})$ through ${ }^{2} \phi$, since ${ }^{2} \psi$ annihilates its image, and since $\mathcal{T}$ is locally trivial we apply exactness at $\mathbf{H}^{1}(X, \mathcal{G})$ (inductively) to see that this in fact defines an $\mathcal{H}$-gerbe. Clearly we have $\delta^{2} \circ{ }^{1} \psi=1$; we also have ${ }^{2} \phi \circ \delta^{2}=1$, by producing a map $\delta^{2}(\mathcal{T}) \rightarrow \mathscr{G}_{\phi}^{0}$ sending $(\widetilde{\mathcal{T}}, f)$ to $\widetilde{\mathcal{T}}$, a $\mathscr{G}$-torsor.

Except for exactness at $\mathbf{H}^{2}(X, \mathcal{G})$, which we prove in a separate lemma, this proposition is not important to the rest of the paper, so we omit the further details.

Lemma I.1.8. Every trivialization of ${ }^{2} \psi(\mathscr{G})$ is induced by an isomorphism $\mathscr{G} \cong$ ${ }^{2} \phi(\mathscr{K})$ for a $\mathcal{K}$-gerbe $\mathscr{K}$, and likewise for torsors.

Proof. If we are given a map $f: \mathscr{G} \rightarrow \mathscr{Q}_{\psi}^{0}$, let $\mathscr{K}$ be the fiber $f^{-1}\left(\mathcal{Q}^{0}\right)$. This is evidently a sheaf of categories; $\mathscr{H}^{1}(X, \mathcal{K})$ acts on it through ${ }^{2} \phi$ because ${ }^{2} \psi$ kills the image of that map, and if $\mathscr{G}$ is trivial then (inductively) by the torsor version of the lemma this fiber is just $\mathscr{H}^{1}(X, \mathcal{K})$, so $\mathscr{K}$ is a $\mathcal{K}$-gerbe. By definition, it admits a map to $\mathscr{G}$, which induces an equivalence ${ }^{2} \phi(\mathscr{K})=\mathscr{G}$, as desired.

Further evidence of the "correctness" of this theory is given by the connection with cohomology: 
Proposition I.1.9. There is a natural identification of equivalence classes of objects in $\mathbf{H}^{2}(X, \mathcal{G})$ with sheaf $\check{C}$ ech cohomology classes in $H^{2}(X, \mathcal{G})$ (and likewise for $H^{1}$ ) which agrees with the product operations, change of group, and long exact sequences.

Since this proposition is not essential for the rest of the paper, we will not give the complete details of the proof.

Proof. Here is how the cohomology class of a gerbe is obtained: for any gerbe $\mathscr{G}$, choose an open cover of $X$ by neighborhoods $U_{i}$ such that $\left.\mathscr{G}\right|_{U}$ is trivial, and pick trivializations $t_{i}$; on $U_{i j}=U_{i} \cap U_{j}$, we have $t_{i j}=t_{j}^{-1} t_{i} \in \mathbf{H}^{1}\left(U_{i j}, \mathcal{G}\right)$, and hence the differences are locally trivial, so we refine the $U_{i}$ to assume that they are totally trivial; we pick trivializations. Then we have on $U_{i j k}=U_{i} \cap U_{j} \cap U_{k}$ two trivializations of $t_{i j k}=t_{i j} t_{j k} t_{k i}$ : the given one and the natural one coming from cancellation. They thus differ by an element of $\mathcal{G}\left(U_{i j k}\right)$ which is easily seen to be a Cech 2-cocycle. One checks that this cocycle depends on the choices of trivializations and the cover $U_{i}$ only up to coboundary, so gives a well-defined cohomology class.

Conversely, given an open cover and a 2-cocycle $t_{i j k}$, for any neighborhood $V$ let $\mathscr{G}_{V}$ be the category of data consisting of $\mathcal{G}$-torsors $\mathcal{T}_{i}$ on the $V \cap U_{i}$ and isomorphisms $\phi_{i j}:\left.\left.\mathcal{T}_{i}\right|_{U_{i j}} \rightarrow \mathcal{T}_{j}\right|_{U_{i j}}$ such that $\phi_{i j} \circ \phi_{j k} \circ \phi_{k i}=t_{i j k}$ as automorphisms of $\left.\mathcal{T}_{i}\right|_{U_{i j k}}$; it is easy to check that this is a $\mathcal{G}$-gerbe and that this construction inverts the one of the previous paragraph. The remaining claims can all be proved using similar reasoning by choosing sufficiently fine trivializations of the gerbes involved.

Our remaining goal for this section is to prove the existence of pullback 2-functors of gerbes along maps of spaces, as for sheaves. This entails a great deal of 2categorical technicalities, some of which we will leave to the reader.

We may rephrase the above lemma in a more inductive way using the concept of a "Čech 1-cocycle with values in torsors". By definition, such a thing (on $X$, for a sheaf of groups $\mathcal{G}$ ) is given by an open cover $\left\{U_{i}\right\}$ of $X$ together with torsors $\mathcal{T}_{i j} \in \mathbf{H}^{1}\left(U_{i j}, \mathcal{G}\right)$ and a trivialization $t_{i j k}$ of the "2-coboundary" $\mathcal{T}_{i j k}=\mathcal{T}_{i j} \otimes \mathcal{T}_{j k} \otimes$ $\mathcal{T}_{k i}$ on the triple intersection $U_{i j k}$, satisfying the cocycle condition on quadruple intersections. Such data is equivalent (using the section-picking arguments of the lemma) to a single Čech 2-cocycle, and we are motivated to introduce the following definition:

Definition I.1.10. We set $\mathbf{H}^{1}\left(X, \mathscr{H}^{1}(X, \mathcal{G})\right)$ to be the 2-category such that:

- Its objects are 1-cocycles $\left(\left\{U_{i}\right\},\left\{\mathcal{T}_{i j}\right\},\left\{t_{i j k}\right\}\right)$ in $\mathcal{G}$-torsors.

- A 1-morphism from $\left(\left\{U_{i}\right\},\left\{\mathcal{T}_{i j}\right\},\left\{t_{i j k}\right\}\right)$ to $\left(\left\{U_{i^{\prime}}^{\prime}\right\},\left\{\mathcal{T}_{i^{\prime} j^{\prime}}^{\prime}\right\},\left\{t_{i^{\prime} j^{\prime} k^{\prime}}^{\prime}\right\}\right)$ is a common refinement $\left\{U_{i^{\prime \prime}}^{\prime \prime}\right\}$ of $\left\{U_{i}\right\}$ and $\left\{U_{i^{\prime}}^{\prime}\right\}$ together with torsors $\mathcal{S}_{i^{\prime \prime}}$ on the $U_{i^{\prime \prime}}$ and isomorphisms of torsors $s_{i j}$ as follows. For simplicity of notation we assume $U_{i}=U_{i^{\prime}}^{\prime}=U_{i^{\prime \prime}}^{\prime \prime}$; then $s_{i j}$ is an isomorphism of $\mathcal{T}^{\prime}{ }_{i j} \otimes \mathcal{S}_{i}$ with $\mathcal{T}_{i j} \otimes \mathcal{S}_{j}$ on $U_{i j}$, compatible with the $t_{i j k}$ and $t_{i j k}^{\prime}$. (In other words, it is a "1-cohomology" in torsors.)

- A 2-morphism between two 1-morphisms

$$
\left(\left\{U_{i}\right\},\left\{\mathcal{S}_{i}\right\},\left\{s_{i j}\right\}\right) \text { and }\left(\left\{U_{i^{\prime}}^{\prime}\right\},\left\{\mathcal{S}_{i^{\prime}}^{\prime}\right\},\left\{s_{i^{\prime} j^{\prime}}^{\prime}\right\}\right)
$$

is a common refinement of $\left\{U_{i}\right\}$ and $\left\{U_{i^{\prime}}^{\prime}\right\}$ together with maps of torsors (using the above notational convention) $r_{i}: \mathcal{S}_{i} \rightarrow \mathcal{S}_{i}^{\prime}$ compatible with the $s_{i j}$ and $s_{i j}^{\prime}$. 
Corollary I.1.11. We have $\mathbf{H}^{2}(X, \mathcal{G}) \cong \mathbf{H}^{1}\left(X, \mathscr{H}^{1}(X, \mathcal{G})\right)$.

Proof. We replace $\mathbf{H}^{2}(X, \mathcal{G})$ with the 2 -category $\mathbf{C}$ of triples $\left(\mathscr{G},\left\{U_{i}\right\},\left\{t_{i}\right\}\right)$, where $\mathscr{G}$ is a gerbe, $\left\{U_{i}\right\}$ is an open cover, and $t_{i}$ is a trivialization of $\left.\mathscr{G}\right|_{U_{i}}$. The morphisms are morphisms of $\mathscr{G}$ alone, so $\mathbf{C}$ is equivalent to $\mathbf{H}^{2}(X, \mathcal{G})$. It admits a natural 2functor $F$ to $\mathbf{H}^{1}\left(X, \mathscr{H}^{1}(X, \mathcal{G})\right)$ sending such a triple to

$$
F\left(\mathscr{G},\left\{U_{i}\right\},\left\{t_{i}\right\}\right)=\left(\left\{U_{i}\right\},\left\{\mathcal{T}_{i j}=t_{j}^{-1} t_{i}\right\},\left\{t_{i j k}\right\}\right),
$$

where $t_{i j k}$ is the natural trivialization of $t_{i}^{-1} t_{k} t_{k}^{-1} t_{j} t_{j}^{-1} t_{i}$. One can check that this extends to a tensor 2-functor, essentially surjective by gluing (the comments following Proposition I.1.9). Since two objects are equivalent (and 1-morphisms isomorphic) if and only if they admit a map, to show that $F$ is fully faithful it suffices to show the following two facts:

(1) If $F\left(\mathscr{G},\left\{U_{i}\right\},\left\{t_{i}\right\}\right)$ is equivalent to the identity object, then $\mathscr{G}$ is trivial. This follows by gluing compatible trivializations of $\mathscr{G}$.

(2) The map $\operatorname{Aut}\left(\mathscr{G}^{0},\{X\},\{\operatorname{id}\}\right) \rightarrow \operatorname{Aut}\left(\{X\},\left\{\mathcal{T}^{0}\right\},\{\operatorname{id}\}\right)$ induced by $F$ is an equivalence of categories. This follows by gluing torsors.

Finally, one can go further still. Suppose $f: Y \rightarrow X$ is any map of spaces; then, as we can pull back torsors (being sheaves), we have a pullback functor

$$
f^{*}: \mathbf{H}^{1}\left(X, \mathscr{H}^{1}(X, \mathcal{G})\right) \rightarrow \mathbf{H}^{1}\left(Y, \mathscr{H}^{1}\left(Y, f^{*} \mathcal{G}\right)\right)
$$

and therefore a pullback functor

$$
f^{*}: \mathbf{H}^{2}(X, \mathcal{G}) \rightarrow \mathbf{H}^{2}\left(Y, f^{*} \mathcal{G}\right)
$$

which, by definition, agrees with the pullback of cohomology classes.

\section{I.2. Twisting CATEgories By A GeRBE}

We have already defined the tensor product of two gerbes and the change of groups along a homomorphism in terms of the "stack of homomorphisms". In general, suppose $\mathcal{G}$ is a sheaf of abelian groups and that $\mathscr{H}^{1}(X, \mathcal{G})$ acts on a sheaf of categories $\mathscr{F}$; then for any $\mathcal{G}$-gerbe $\mathscr{G}$, we may form

$$
\mathscr{F}(\mathscr{G})=\mathscr{G} \otimes \mathscr{F}=\mathscr{H} \mathrm{om}\left(\mathscr{G}^{-1}, \mathscr{F}\right),
$$

the stack of $\mathscr{G}$-twisted objects of $\mathscr{F}$. Sometimes, if we denote $\mathbf{F}=\mathscr{F}_{X}$, we will write $\mathscr{G} \otimes \mathbf{F}$ by abuse of notation to mean $\mathscr{F}(\mathscr{G})_{X}$.

We can give two concrete descriptions of a section $s \in \mathscr{F}(\mathscr{G})_{Y}$ of a twisted sheaf of categories:

(1) Using Corollary I.1.11. Suppose that $\mathscr{G}$ is trivialized on an open cover $U_{i}$; then a section $s \in \mathscr{F}(\mathscr{G})_{X}$ is given by sections $s_{i} \in \mathscr{F}_{U_{i}}$ together with isomorphisms $s_{j} \cong \mathcal{T} \otimes s_{i}$ on $U_{i j}$ (using $\otimes$ to denote the action of a torsor on sections of $\mathscr{F})$ satisfying the 1 -cocycle condition with respect to the cocycle constraint of $\mathcal{T}$.

(2) Using Proposition 1.1.9 Suppose that the $U_{i}$ are chosen so that all the transition torsors are trivial and denote by $t_{i j k} \in \mathcal{G}_{U_{i j k}}$ the Cech 2-cocycle corresponding to $\mathscr{G}$; then $s$ is given by $s_{i} \in \mathscr{F}_{U_{i}}$ and isomorphisms $s_{i} \cong s_{j}$ whose coboundary on $U_{i j k}$ is equal to the action of $t_{i j k}$. 
From either of these descriptions, it is clear that if we have a group homomorphism $\phi: \mathcal{G} \rightarrow \mathcal{H}$ and an $\mathcal{H}$-action on $\mathscr{F}$, then for any $\mathcal{G}$-gerbe $\mathscr{G}$ we have a functor $\mathscr{F}(\mathscr{G}) \rightarrow \mathscr{F}\left({ }^{2} \phi \mathscr{G}\right)$, where the first twisting is with respect to $\mathcal{G}$ and the second with respect to $\mathcal{H}$.

Despite the intuitive appeal of (2), it is not as useful as the coarser (1) because sometimes, one does not have the freedom to refine $U$ to the point that the torsors are trivial. For example, the following construction/theorem, whose proof is implicit in the statement:

Proposition I.2.1. Suppose that $\mathscr{F}$ has pushforwards: for any $g: Z \rightarrow Y$ the pullback map $g^{*}: \mathscr{F}_{Y} \rightarrow \mathscr{F}_{Z}$ admits a right adjoint such that $g^{*} g_{*} \rightarrow$ id is an isomorphism of functors, and $g_{*}$ satisfies a projection formula with respect to the action of $\mathscr{H}^{1}(X, \mathcal{G}):$ for any torsor $\mathcal{T}$ on $Y$ and $t \in \mathscr{F}_{Z}$, the natural map

$$
\mathcal{T} \otimes g_{*} t \rightarrow g_{*}\left(g^{*} \mathcal{T} \otimes t\right)
$$

is an isomorphism. Let $f: Y \rightarrow X$ and suppose that the $U_{i}^{0}$ cover $X$, with $U_{i}$ the pullback cover of $Y$; let $\mathscr{G}$ be specified on the $U_{i}^{0}$ as in $(1)$ above. For any $s \in \mathscr{F}(\mathscr{G})_{Y}$ specified with respect to the $U_{i}$, there exists a pushforward $f_{*}(s) \in \mathscr{F}(\mathscr{G})_{X}$, specified by the $f_{*}\left(s_{i}\right)$, extending to a pushforward functor of the above description.

It is with the introduction of the geometric functors that large and small topologies make an appearance; by the difference we mean that large topologies generally include all objects in the site and merely specify covers, whereas the small topologies also restrict the objects. Large topologies are useful for theoretical purposes but not in practice, and we will not use them outside this prologue. Our reason for using them here is that the existence of pullbacks of sheaves of categories is a theorem of type "small to large"; it says that a sheaf of categories and, in particular, a gerbe on a small topology over an object $X$ naturally induces a unique one on the corresponding large topology. Then the geometric functors have the following interpretation:

Let $f: Y \rightarrow X$ be a map and $\mathscr{G}$ be a gerbe on the small topology of $X$, while $\mathscr{F}$ is a sheaf of categories on the large topology (we have in mind here that of perverse sheaves). We will call $\left.\mathscr{F}\right|_{X}$ and $\left.\mathscr{F}\right|_{Y}$ its restrictions to the small sites. We employ the above extension of $\mathscr{G}$ to the large topology in order to form $\mathscr{F}(\mathscr{G})$, and in this language we find that there is a pullback functor of sections twisted on the small categories,

$$
f^{*}:\left.\left.\mathscr{F}\right|_{X}(\mathscr{G})_{X} \rightarrow \mathscr{F}\right|_{Y}\left(f^{*} \mathscr{G}\right)_{Y}
$$

and possibly a pushforward functor going the other way.

The stack $\mathscr{F}$ may enjoy many categorical properties, among them its categories of sections being abelian or triangulated. We will term a sheaf of such categories one in which the pullback maps respect all the structures imposed on the categories; for example, a sheaf of abelian categories is one in which all pullbacks are exact, and one of triangulated categories has triangulated pullbacks. This necessitates the following comments.

Sheaves of abelian categories. The usual abelian categories of sheaves are not sheaves of abelian categories in the large topologies, because general pullbacks tend only to be right exact. This is true in particular for perverse sheaves (where pullbacks are not even right exact). However, we will only ever need the large topology for the theoretical purposes of this section, and in the rest of the work the 
classical site whose objects over a space $X$ are simply open sets in $X$ will suffice, and for such a small topology, pullbacks are exact. If, as in the case of this particular sheaf of abelian categories, tensoring with a $\mathcal{G}$-torsor is exact, then $\mathscr{F}(\mathscr{G})$ is again a sheaf of abelian categories in the obvious way.

Twisted derived category. The fibered category of derived categories of sheaves on $X$ is not a stack because it does not enjoy descent (also, there is ambiguity as to what its pullbacks are, since there are two kinds). Some of our constructions in Part IV require mention of functors that are a priori merely derived, so in order to handle this rigorously we introduce the sheaf $\mathscr{D}$ of categories to be the sheafification of the aforementioned fibered category; the operation of "associated stack" is given in Gir71, §II.2] and associates with a fibered category $\mathscr{F}$ the universal stack whose sections are locally isomorphic to those of $\mathscr{F}$.

Then $\mathscr{D}$ has not only $f^{*}$ but also $f^{!}$, as well as the corresponding lower-indexed adjoints. If in the topology we use, all open covers are of a class of morphisms for which $f^{*}=f^{!}$, then it is a stack for both structures of fibered category; this will be the case either for the classical or the étale topologies, though we only use the former. By definition, the duality functor is either a map $\mathbb{D}:\left(\mathscr{D}^{*}\right)^{\text {op }} \rightarrow \mathscr{D}^{\text {! }}$ or in the other direction, where the superscript indicates which kind of pullbacks $\left(f^{!}\right.$or $\left.f^{*}\right)$ are the cartesian morphisms in the fibered category. In this situation, we will be taking $\mathcal{G}$ to be the constant sheaf on a complex variety with values in the multiplicative group of some field of characteristic zero, so its torsors are local systems, and tensor product with these is respected by $\mathbb{D}$. We conclude that all of $f^{*}, f^{!}, f_{*}, f_{!}$, and $\mathbb{D}$ have twisted analogues.

Twisted $t$-structures. Objects of $\mathscr{D}(\mathscr{G})_{X}$ are not important for us insofar as the ones we will use will always turn out to be twisted perverse sheaves. In order to effect this, we will need to impose the perverse $t$-structure on $\mathscr{D}(\mathscr{G})$, which will simply be piecewise: a section $s$ is in $p \mathscr{D}(\mathscr{G})_{X}^{\leqslant 0}$ if $u_{i}^{*} s$ is, where the $u_{i}: U_{i} \rightarrow X$ are a trivializing open cover for $\mathscr{G}$, and likewise will be in $p \mathscr{D}(\mathscr{G})_{X}^{\geqslant 0}$ if $u_{i}^{!} s$ is (or equivalently, if $\left.\mathbb{D}(s) \in{ }^{p} \mathscr{D}(\mathscr{G})_{X}^{\leqslant 0}\right)$. This is sensible since twisting by a local system respects perversity; the core of this $t$-structure is the twisted category of perverse sheaves (that is, taking the core and twisting by $\mathscr{G}$ commute as operations).

\section{I.3. EquiVARIANCE AND MULTIPLICATIVITY}

The two most important structures on gerbes, for us, are equivariance and multiplicativity. As before, let $X$ be any space, $Y \rightarrow X$ any map, and let $G$ be an $X$-group with multiplication map $m: G \times{ }_{X} G \rightarrow G$. Since all our spaces are relative to $X$, we will drop the subscripts and just write $\times$.

The definitions. An equivariant gerbe is the analogue of a $G$-invariant map between spaces with a $G$-action.

Definition I.3.1. Let $a: G \times Y \rightarrow Y$ be a $G$-action, let $\mathscr{G}$ be a gerbe on $Y$, and let pr: $G \times Y \rightarrow Y$ be the projection map. A $G$-equivariance structure for $\mathscr{G}$ is the following data:

(1) (Equivariance) An equivalence of gerbes on $G \times Y$ :

$$
\mathrm{Eq}: a^{*} \mathscr{G} \rightarrow \mathrm{pr}^{*} \mathscr{G} \text {. }
$$


(2) (Compatibilities) Isomorphisms:

$$
\begin{gathered}
\text { As: } \mathrm{Eq}_{11}=\left(\operatorname{pr}_{G \times Y}^{*} \mathrm{Eq}\right) \circ\left((G \times a)^{*} \mathrm{Eq}\right) \rightarrow(m \times Y)^{*} \mathrm{Eq} \\
\mathrm{Id}: \mathrm{id} \rightarrow(1 \times Y)^{*} \mathrm{Eq}
\end{gathered}
$$

referring to the following diagrams: for As (over $G \times G \times Y$ ),

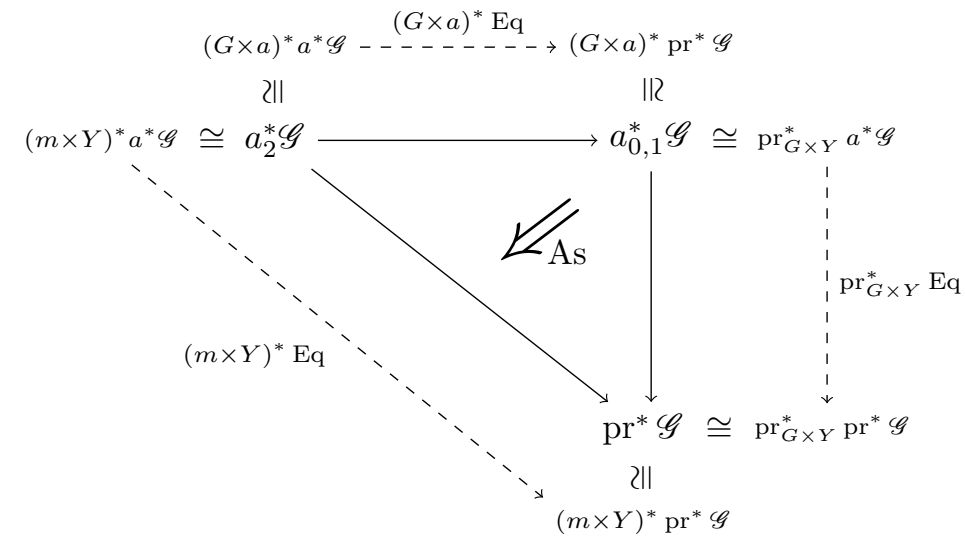

and for Id,

$$
(1 \times Y)^{*} \mathrm{Eq}:(1 \times Y)^{*} a^{*} \mathscr{G}=\mathscr{G} \rightarrow(1 \times Y)^{*} \operatorname{pr}^{*} \mathscr{G}=\mathscr{G} .
$$

(3) (Cocycle condition) As is required to satisfy the 2-cocycle condition on $G \times G \times G \times X$, which we will not write but which is an identity on As corresponding to the two ways of using associativity to rewrite the parenthesized expression:

$$
\begin{aligned}
& ((w x) y) z=(w(x y)) z=w((x y) z)=w(x(y z)) \\
& ((w x) y) z=\quad(w x)(y z) \quad=w(x(y z)) .
\end{aligned}
$$

Likewise, we may speak of the gerbe analogue of a group homomorphism:

Definition I.3.2. Let $\mathscr{M}$ be a gerbe on $G$ itself; then a multiplicative structure for $\mathscr{M}$ is an equivalence,

$$
\mathrm{Mul}: m^{*} \mathscr{M} \rightarrow \mathscr{M} \otimes \mathscr{M},
$$

with associativity and identity constraints as for equivariance. This structure is commutative if, in addition, denoting by sw: $G \times G \rightarrow G \times G$ the coordinate swap, we are given an isomorphism between

$$
\mathrm{sw}^{*}(\mathscr{M} \otimes \mathscr{M}) \stackrel{\mathrm{sw}^{*} \mathrm{Mul}^{-1}}{\longrightarrow} \mathrm{sw}^{*} m^{*} \mathscr{M}=m^{*} \mathscr{M} \stackrel{\mathrm{Mul}}{\longrightarrow} \mathscr{M} \otimes \mathscr{M}
$$

and the natural equivalence of the first and last terms.

Given such an $\mathscr{M}$, the structure of twisted equivariance on a gerbe $\mathscr{G}$ on $Y$ is an equivalence,

$$
\alpha: a^{*} \mathscr{G} \rightarrow \mathscr{M} \otimes \mathscr{G},
$$

with an associativity constraint As and identity constraint Id as before, where As and Id make reference to the associativity and identity constraints of both $\mathrm{Mul}$ and of the tensor product of gerbes. By definition, an equivariance structure is just twisted equivariance with respect to the trivial multiplicative gerbe (see Definition I.3.4), and any multiplicative gerbe is also twisted equivariant on both sides, or equivalently, $G \times G^{\mathrm{op}}$-twisted-equivariant. 
This definition can be understood concretely in the situation where $G$ is a discrete group relative to a base space $X$, and we consider it as its group of connected components: " $G=X \times G$ ".

Definition I.3.3. Let $G$ be a group (in sets). A multiplicative gerbe on $X \times G$ is the data of, for each $g \in G$, a gerbe $\mathscr{G}^{g}$ on $X$; for each pair $g, h$ an equivalence $\mathscr{G}^{g h} \cong \mathscr{G}^{g} \otimes \mathscr{G}^{h} ;$ for each triple $g, h, k$, we have an isomorphism of

$$
\mathscr{G}^{g} \otimes\left(\mathscr{G}^{h} \otimes \mathscr{G}^{k}\right) \cong \mathscr{G}^{g h k} \cong\left(\mathscr{G}^{g} \otimes \mathscr{G}^{h}\right) \otimes \mathscr{G}^{k}
$$

with the natural associativity of gerbe products, satisfying the cocycle condition. If $G$ is commutative, we also require an isomorphism of equivalences between the following,

$$
\mathscr{G}^{g} \otimes \mathscr{G}^{h} \cong \mathscr{G}^{g h}=\mathscr{G}^{h g} \cong \mathscr{G}^{h} \otimes \mathscr{G}^{g}
$$

and the natural commutativity of the tensor product of gerbes.

The most obvious examples of these structures are of course the trivial ones. For later use we state the definition in a manner which is in fact not entirely trivial. Recall that all our spaces are over a fixed base $X$.

Definition I.3.4. We say that a gerbe $\mathscr{G}$ on $Y$ is $X$-trivialized if it is given descent data to $X$; i.e., if we denote by $p$ the structure map to $X$, we have an equivalence $\mathscr{G} \cong p^{*} \mathscr{G}_{X}$ constituting 2 -descent data. Any $X$-trivialized gerbe $\mathscr{M}$ on $G$ has a natural multiplicative structure, called the trivial multiplicative structure, as follows: if $\mathscr{M}_{X}=\left.\mathscr{M}\right|_{1_{X}}$ is the gerbe on $X$ descending $\mathscr{M}$ (the restriction to the identity section), then on $G \times_{X} G$ we have (overloading the notation $p$ ):

$$
m^{*} \mathscr{M} \cong p^{*} \mathscr{M}_{X} \cong p^{*} \mathscr{M}_{X} \nabla_{X} p^{*} \mathscr{M}_{X} \cong \mathscr{M}_{X} \mathscr{M},
$$

with $\otimes_{X}$ denoting the outer tensor product relative to the fiber product $G \times_{X} G$. Similar measures express pullbacks to higher fibered Cartesian powers of $G$ and fill out the multiplicative structure. Likewise, we have the trivial equivariance structure on an $X$-trivialized gerbe $\mathscr{G}$ on $Y$.

Lemma I.3.5. Let $\mathscr{G}$ be a $X$-trivial gerbe on $Y$; then the $G$-equivariance structures on $\mathscr{G}$ are identified with multiplicative torsors on the $Y$-group $G \times_{X} Y$.

Proof. Referring to the notation of Definition I.3.1, both $a^{*} \mathscr{G}$ and $\operatorname{pr}^{*} \mathscr{G}$ are identified with $p^{*} \mathscr{G}_{X}$, where $p: G \times_{X} Y \rightarrow X$ is the structure map. Therefore, the equivalence $\mathrm{Eq}$ is identified with a torsor on $G \times_{X} Y$. The isomorphism As on $G \times_{X} G \times_{X} Y$, which is the same as $\left(G \times_{X} Y\right) \times_{Y}\left(G \times_{X} Y\right)$, then expresses

$$
\mathrm{Eq} \otimes_{Y} \mathrm{Eq} \cong m_{G \times_{X} Y}^{*} \mathrm{Eq},
$$

where $m_{G \times X Y}$ is the same as $m \times Y$ but considered as the group operation on $G \times_{X} Y$. Likewise, the unwritten cocycle condition expresses associativity of this multiplicative structure.

Descent properties. Outside of this part, we will use equivariance only as "that which gives descent":

Lemma I.3.6. Suppose $\mathcal{G}$ is a sheaf of groups on $Y$, and let $\pi: \widetilde{Y} \rightarrow Y$ be a $G$-torsor. Then $G$-equivariant $\pi^{*}(\mathcal{G})$-gerbes on $\tilde{Y}$ are equivalent to $\mathcal{G}$-gerbes on $Y$. 
Proof. One direction is simple: if $\mathscr{G}$ is a gerbe on $Y$, then the natural equivalences of the pullback make $\pi^{*} \mathscr{G}$ an equivariant gerbe on $\tilde{Y}$.

Conversely, we will descend gerbes in the form given by Corollary I.1.11. We claim that if $\mathscr{G}$ is a $G$-equivariant gerbe on $\tilde{Y}$, then there is an open cover $\left\{U_{i}\right\}$ of $Y$ and trivializations of $\left.\mathscr{G}\right|_{\pi^{-1}\left(U_{i}\right)}$ whose transition torsors are themselves $G$ equivariant. Knowing this, the lemma follows from the descent of $G$-equivariant sheaves (or the analogous argument for torsors).

To prove the claim, we begin with any cover $\left\{U_{i}\right\}$ trivializing $\tilde{Y}$, and pick trivializations $\pi^{-1}\left(U_{i}\right) \cong G \times U_{i}$. Then the restrictions $\left.\mathscr{G}\right|_{1 \times U_{i}}$, being gerbes on the $U_{i}$, are locally trivial and we may refine the cover so that they are trivial. It therefore suffices to prove that:

(1) If $\widetilde{Y}=G \times Y$ is the trivial torsor, then $\mathscr{G}=\pi^{*}\left(\left.\mathscr{G}\right|_{1 \times Y}\right)$. Furthermore, if $\left.\mathscr{G}\right|_{1 \times Y}$ is trivial, then $\mathscr{G}$ is equivariantly trivial.

(2) Writing $V_{i}=\left.\widetilde{Y}\right|_{U_{i}}$ and $V_{j}=\left.\widetilde{Y}\right|_{U_{j}}$, if $\left.\mathscr{G}\right|_{V_{i}},\left.\mathscr{G}\right|_{V_{j}}$ are equivariantly trivialized then the transition 1-morphism is an equivariant $\mathcal{G}$-torsor.

(3) Given $U_{i}, U_{j}$, and $U_{k}$, if $\mathscr{G}$ is trivialized over all of these opens and if the transition torsors are also equivariantly trivialized, then the 2-morphism (section of $\mathcal{G}$ ) witnessing the cocycle condition is $G$-invariant.

We consider (1). When $\tilde{Y} \cong G \times Y$ is trivialized, the action of $G$ is by multiplication $m$ on the first factor, and on $G \times G \times Y$ we have the equivariance datum $\mathrm{Eq}: \operatorname{pr}_{G \times Y}^{*} \mathscr{G} \cong(m \times \mathrm{id})^{*} \mathscr{G}$. If we restrict to the slice $G \times 1 \times Y$ we get an equivalence between $\pi^{*}\left(\left.\mathscr{G}\right|_{1 \times Y}\right)$ and $\mathscr{G}$, as desired.

Suppose that $\left.\mathscr{G}\right|_{1 \times Y}$ is trivial, so that this is a trivialization of $\mathscr{G}$; we claim that it extends to a trivialization of the equivariance structure. Note that now Eq is an automorphism of the trivial gerbe on $G \times G \times Y$, hence a $\mathcal{G}$-torsor. Indeed, considering the first of the (Compatibilities) of Definition I.3.1 restricted to $G \times$ $G \times(1 \times Y) \subset G \times G \times \tilde{Y}$, we get $\mathrm{Eq} \otimes \mathrm{Eq} \cong \mathrm{Eq}$, so $\mathrm{Eq}$ is trivialized. By definition, the unit map Un is also trivialized.

Now we consider (2). Denote by $\phi$ the transition torsor from $\left.\mathscr{G}\right|_{V_{i}} \cong \mathscr{G}^{0}$ to $\left.\mathscr{G}\right|_{V_{j}} \cong \mathscr{G}^{0}$, so that on $G \times \widetilde{Y}$, the pullback $\operatorname{pr}_{\widetilde{Y}}^{*} \mathscr{G}$ is glued from two trivial gerbes on $G \times V_{i}$ and $G \times V_{j}$ by $\operatorname{pr}_{\widetilde{Y}}^{*} \phi$, and likewise for $a^{*} \mathscr{G}$. By assumption, the equivariance data $\mathrm{Eq}$ is trivial on both $G \times V_{i}$ and $G \times V_{j}$, and therefore consists merely of a 2-isomorphism identifying $\mathrm{Eq} \circ a^{*} \phi$ and $\mathrm{pr}_{V_{i} \cap V_{j}}^{*} \phi \circ \mathrm{Eq}$, where the Eq terms may be neglected. This is the equivariance structure for $\phi$. The proof for (3) is similar.

The lemma can be rephrased informally as "a $G$-equivariant gerbe on $Y$ is the same as a gerbe on the stack $Y / G$ ". More precisely, we make the following definition:

Definition I.3.7. Let $G$ act on $Y$; a gerbe on the quotient stack $Y / G$ is the following data: for every space $S, G$-torsor $\widetilde{S}$, and $G$-map $s: \widetilde{S} \rightarrow Y$, a gerbe $\mathscr{G}_{\widetilde{S}, s}$ on $S$. Furthermore, if $f: T \rightarrow S$ and we write $\widetilde{T}=f^{*} \widetilde{S}=\widetilde{S} \times{ }_{S} T$ with its induced map $t: \widetilde{T} \rightarrow Y$, then we must have equivalences $f^{*} \mathscr{G}_{\widetilde{S}, s} \cong \mathscr{G}_{\widetilde{T}, t}$. If $U \rightarrow T \rightarrow S$ is a triple of maps, then both equivalences from $S$ to $U$ must be isomorphic, and these isomorphisms must satisfy a cocycle condition for triple nestings.

Corollary I.3.8. Let $G$ act on $Y$ and let $\mathscr{G}$ be a gerbe on $Y$. A structure of $G$-equivariance on $\mathscr{G}$ is equivalent to giving a gerbe on the quotient stack as in 
Definition I.3.7, such that if for $S$ and $s: \widetilde{S} \rightarrow Y$ as there, writing $\pi: \widetilde{S} \rightarrow S$ for the torsor structure map, we also have:

(1) Equivalences $s^{*} \mathscr{G} \cong \pi^{*} \mathscr{G}_{\widetilde{S}, s}$.

(2) For $f: T \rightarrow S$ and $\widetilde{T}=f^{*} \widetilde{S}$ as above (and denoting $f: \widetilde{T} \rightarrow \widetilde{S}$ ), 2isomorphisms making the triangle of maps of gerbes on $\widetilde{T}$ commute. These isomorphisms must satisfy the cocycle condition for a triple of maps.

All of the notions of equivariance, (commutative) multiplicativity, triviality, and descent are related via the following proposition:

Proposition I.3.9. Let $\widetilde{G}$ be a group, $G$ a subgroup, $\mathscr{M}$ a multiplicative gerbe on $\widetilde{G}$. Then to give a multiplicative trivialization of $\left.\mathscr{M}\right|_{G}$ is the same as to give an $\mathscr{M}$-twisted equivariant gerbe $\mathscr{G}$ on $Y=\widetilde{G} / G$ descending $\mathscr{M}$ :

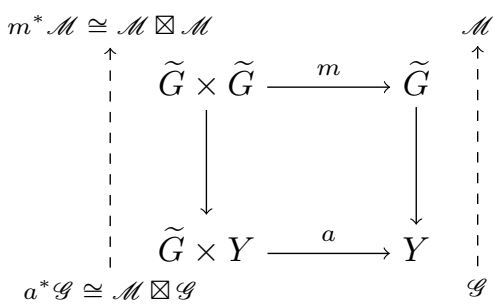

Proof. The trivialization of $\left.\mathscr{M}\right|_{G}$ gives the data of $G$-equivariance for $\mathscr{M}$, via

$$
\left.\left.m\right|_{G \times \widetilde{G}} ^{*} \mathscr{M} \cong \mathscr{M}\right|_{G} \otimes \mathscr{M} \cong \mathscr{G}^{0} \nabla \mathscr{M} \cong \operatorname{pr}_{\widetilde{G}}^{*} \mathscr{M} .
$$

Thus, by Lemma I.3.6, $\mathscr{M}$ descends to a gerbe $\mathscr{G}$ on $Y=\widetilde{G} / G$. By associativity of multiplication, the multiplicative structure on $\mathscr{M}$ descends to twisted equivariance of $\mathscr{G}$ over $\widetilde{G} \times Y$. Note that this reduces to just $G$-equivariance when the action is restricted to $G \subset \widetilde{G}$, since $\left.\mathscr{M}\right|_{G}$ is explicitly trivialized. Conversely, if $\mathscr{G}$ is an equivariant gerbe on $Y$ whose equivariance structure descends the multiplicative structure of $\mathscr{M}$, then over $1=1_{\widetilde{G}} / G \in Y$, the isomorphism

$$
\left.\left.\left.\left.\left.\left.\mathscr{M}\right|_{G} \otimes \mathscr{G}\right|_{1} \cong a\right|_{G \times 1} ^{*} \mathscr{G}\right|_{1} \cong \operatorname{pr}_{G \times 1}^{*} \mathscr{G}\right|_{1} \cong \mathscr{G}\right|_{1}
$$

cancels to a trivialization of $\left.\mathscr{M}\right|_{G}$.

Equivariant twisted objects. Now we complicate the picture by describing the same structures on sections of a sheaf of categories twisted by a gerbe.

Definition I.3.10. Let $G$ act on $Y$, let $\mathscr{G}$ be an equivariant gerbe on $Y$, and let $\mathscr{F}$ be a sheaf of categories of $Y$ (the latter in the large topology); we define the notion of $G$-equivariance of an object $s \in \mathscr{F}(\mathscr{G})_{Y}$ to be the following. Recall the pullback functors

$$
\begin{aligned}
a^{*}:\left.\mathscr{F}\right|_{Y}(\mathscr{G})_{Y} & \left.\rightarrow \mathscr{F}\right|_{G \times Y}\left(a^{*} \mathscr{G}\right)_{G \times Y}, \\
\operatorname{pr}^{*}:\left.\mathscr{F}\right|_{Y}(\mathscr{G})_{Y} & \left.\rightarrow \mathscr{F}\right|_{G \times Y}\left(\operatorname{pr}^{*} \mathscr{G}\right)_{G \times Y},
\end{aligned}
$$

and an equivalence $\mathrm{Eq}: a^{*} \mathscr{G} \rightarrow \operatorname{pr}^{*} \mathscr{G}$. The equivariant structure on $s$ is then:

(1) (Equivariance) An isomorphism

$$
\varepsilon:(\mathrm{Eq} \otimes \mathrm{id})\left(a^{*} s\right) \rightarrow \mathrm{pr}^{*} s
$$

of sections of $\left.\operatorname{pr}^{*} \mathscr{G} \otimes \mathscr{F}\right|_{G \times Y}$. 
(2) (Identity condition) $\varepsilon$ must agree with the unit

$$
(1 \otimes Y)^{*} \varepsilon \circ(\mathrm{Id} \otimes \mathrm{id})=\mathrm{id}
$$

as maps from $s$ to itself.

(3) (Cocycle condition) The following diagram must commute:

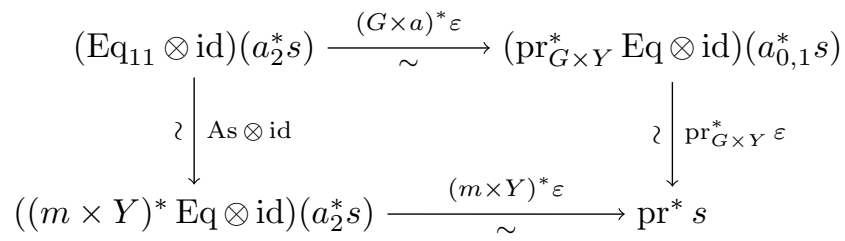

where the vertices are all sections of $\left.\operatorname{pr}^{*} \mathscr{G} \otimes \mathscr{F}\right|_{G \times G \times Y}$ :

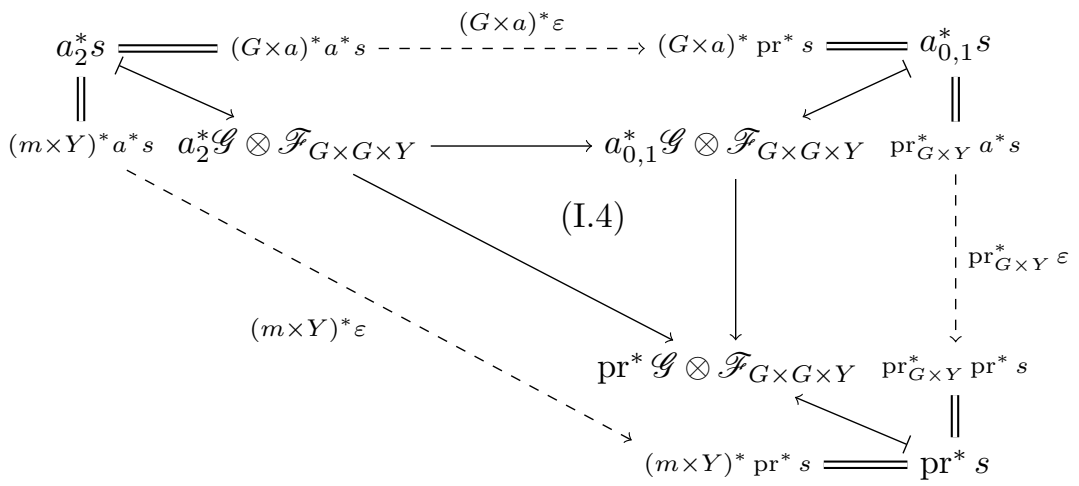

Constructions and restrictions. Equivariance data becomes dramatically simplified when the group in question acts transitively.

Lemma I.3.11. Let $X$ be a connected space, $G$ a connected $X$-group, $A$ an abelian group, $\mathcal{M}$ an $A$-torsor on $G$ trivialized over the unit section $\{1\}=1_{X}$; then $\mathcal{M}$ has at most one multiplicative structure. Furthermore, if $m: G \times{ }_{X} G \rightarrow G$ and we have an isomorphism $m^{*} \mathcal{M} \cong \mathcal{M} \otimes_{X} \mathcal{M}$, for this to constitute a multiplicative structure it suffices that its restriction to $\{1\} \times G$ be the identity.

Proof. Any multiplicative structure on $\mathcal{M}$ is an isomorphism $\mu: m^{*} \mathcal{M} \cong \mathcal{M} \nabla_{X} \mathcal{M}$ which is the identity map over $G \times\{1\}$ and $\{1\} \times G$. Clearly the product of two multiplicative torsors is again multiplicative, as is the inverse, so it suffices to show that if $\mathcal{M}$ is trivial, then $\mu$ is the identity map, identifying both sides with the trivial $A$-torsor $\mathcal{T}^{0}$ on $G \times_{X} G$. Picking some section of $m^{*} \mathcal{M}$ we have a noncanonical identification of $\mu$ with an element of $A$ since $G$ is connected, and since $\left.\mu\right|_{\{1\} \times G}=1$, that element is 1 , as desired.

For the second claim, we must verify associativity: that both the induced isomorphisms of $(m \times \mathrm{id})^{*} m^{*} \mathcal{M}=(\mathrm{id} \times m)^{*} m^{*} \mathcal{M}$ with $\mathcal{M}^{\otimes 3}$ are equal. Let $\mathcal{T}$ be the difference between the two torsors, thus given two trivializations which must necessarily differ by some $a \in A$. Restricting to $\{1\} \times G \times\{1\}$ we find that both are equal, so $a=1$, as desired.

Lemma 1.3.12. Let $X$ be a connected space, $G$ a connected $X$-group acting relatively transitively on the $X$-space $Y$; let $k$ be a field and $\mathscr{G}$ be a $k^{*}$-gerbe on $Y$ and $\mathcal{F}$ a $\mathscr{G}$-twisted locally constant sheaf of $k$-vector spaces on $Y$. If $\mathscr{G}$ and $\mathcal{F}$ are 
$G$-equivariant, and if for some section $X \subset Y$ we have $\operatorname{Stab}_{G}(X)$ connected, then both $\mathscr{G}$ and $\mathcal{F}$ are $X$-trivial.

Proof. Picking $X \subset Y$ and taking $H=\operatorname{Stab}_{G}(X)$, we have $Y \cong G / H$; let $\pi: G \rightarrow$ $G / H$ be the quotient. Then $\pi^{*} \mathscr{G}$ is an $G$-equivariant gerbe on $G$ and is thus $X$ trivial by Lemma I.3.6 likewise, $\pi^{*} \mathcal{F}$ is $X$-trivial. To show that these trivializations descend to $Y$, it suffices to show that the natural $H$-equivariance structures on $\pi^{*} \mathscr{G}$ and $\pi^{*} \mathcal{F}$ are the trivial ones.

By Lemma I.3.5 the $H$-equivariance of $\pi^{*} \mathscr{G}$ is the same as a multiplicative $k^{*}$ torsor Eq on the $G$-relative group $H \times_{X} G$ (where $G$ is considered without its group structure). Likewise, the equivariance of $\pi^{*} \mathcal{F}$ is an isomorphism

$$
m_{H}^{*}\left(\pi^{*} \mathcal{F}\right) \cong \mathrm{Eq} \otimes \operatorname{pr}_{G}^{*}\left(\pi^{*} \mathcal{F}\right) .
$$

In the notation of Definition I.3.4, both pullbacks are $p^{*} \mathcal{F}_{X}$; since the action of $k^{*}$ torsors on locally constant sheaves of $k$-vector spaces is faithful, this isomorphism makes Eq a trivial torsor. It is therefore a fortiori $G$-trivial (on $H \times_{X} G$ ) so by Lemma I.3.11 it is multiplicatively trivial since $H$ is connected. Consequently, $\pi^{*} \mathscr{G}$ is indeed $H$-equivariantly trivial, as desired.

Since Eq is trivial, in (I.5) the isomorphism is given by a section of $\mathrm{GL}_{n}(k)$ ( $n$ being the rank of $\mathcal{F}$ ) over $H \times G$ which is the identity on $\{1\} \times G$. Since $H$ is connected, this section is equal to 1 . Thus, $\mathcal{F}$ is $X$-trivially $K$-equivariant, as desired.

We can apply descent along torsors to the construction of "twisted pullbacks".

Definition I.3.13. Let $G$ act on $Y$ and let $\widetilde{X} \rightarrow X$ be a $G$-torsor; set $\widetilde{X}_{Y}=$ $\widetilde{X} \times{ }^{G} Y=(\widetilde{X} \times Y) / G$, so $\widetilde{X} \times Y$ is a $G$-torsor over $\widetilde{X}_{Y}$. The twisted pullback of a $G$-equivariant gerbe $\mathscr{G}$ on $Y$ is the gerbe on $\widetilde{X}_{Y}$ descending $\operatorname{pr}_{Y}^{*} \mathscr{G}$ from $\tilde{X} \times Y$. When $\tilde{X}$ is understood, we will denote the twisted pullback by $\widetilde{\mathscr{G}}$. The same procedure defines the twisted pullback of an equivariant section of some sheaf of categories twisted by $\mathscr{G}$.

Equivariance of gerbes and their twisted objects is compatible with pullback along equivariant maps, as formalized in the following proposition.

Proposition I.3.14. Let $G$ act on $X$ and $H$ on $Y$, let $\phi: H \rightarrow G$ be a homomorphism, let $f: X \rightarrow Y$ be $(G, H)$-equivariant, let $\mathscr{G}$ be a $G$-equivariant gerbe on $X$, and let $\mathscr{F}$ be a sheaf of categories on $X$ with an action of $\mathscr{H}^{1}(Y, \mathcal{G})$. Then $f^{*} \mathscr{G}$ has an $H$-equivariance structure, and for any $G$-equivariant object $\left.s \in \mathscr{F}\right|_{X}(\mathscr{G})_{Y}$, there is an associated structure of $H$-equivariance on $\left.f^{*} s \in f^{*} \mathscr{F}\right|_{Y}\left(f^{*} \mathscr{G}\right)_{Y}$.

\section{I.4. Twisting Structures By A Gerbe}

We now consider the interaction between twisting a sheaf of categories and structures defined on that sheaf. The basic theme is that if a sheaf of categories possesses a structure, a necessary and sufficient condition for it to pass to the twisting by some gerbe is that it be preserved by the action of torsors.

Twisted pairings. One simple example is the twisting of a pairing: suppose we have three sheaves of categories $\mathscr{F}_{1}, \mathscr{F}_{2}, \mathscr{F}_{3}$ and a pairing (e.g., tensor product)

$$
P: \mathscr{F}_{1} \times \mathscr{F}_{2} \rightarrow \mathscr{F}_{3} .
$$


Definition I.4.1. Let the $\mathscr{F}_{i}$ have actions of $\mathscr{H}^{1}(X, \mathcal{G})$; then the structure of $\mathcal{G}$-bi-equivariance is, for any sections $s \in\left(\mathscr{F}_{1}\right)_{U}, t \in\left(\mathscr{F}_{2}\right)_{U}$, and any $\mathcal{G}$-torsor $\mathcal{T}$, natural isomorphisms

$$
P(\mathcal{T} \otimes s, t) \cong \mathcal{T} \otimes P(s, t), \quad P(s, \mathcal{T} \otimes t) \cong \mathcal{T} \otimes P(s, t) .
$$

Thus, for any $\mathcal{G}$-torsors $\mathcal{T}_{1}, \mathcal{T}_{2}$, we have

$$
P\left(\mathcal{T}_{1} \otimes s, \mathcal{T}_{2} \otimes t\right)=\left(\mathcal{T}_{1} \otimes \mathcal{T}_{2}\right) \otimes P(s, t) .
$$

If $\mathscr{G}_{1}$ and $\mathscr{G}_{2}$ are two $\mathcal{G}$-gerbes, then the action of $\mathscr{H}^{1}(X, \mathcal{G})$ on their tensor product is through the tensor product of torsors, so we have the following result:

Proposition I.4.2. If $P$ is $\mathcal{G}$-biequivariant, it admits a twisting:

$$
\widetilde{P}: \mathscr{F}_{1}\left(\mathscr{G}_{1}\right) \times \mathscr{F}_{2}\left(\mathscr{G}_{2}\right) \rightarrow \mathscr{F}_{3}\left(\mathscr{G}_{1} \otimes \mathscr{G}_{2}\right) .
$$

This construction is a special case of a more general operation in which we have two sheaves of groups $\mathcal{G}_{i}$ whose torsors act on the $\mathscr{F}_{i}(i=1,2)$, while $\mathscr{F}_{3}$ has an action of

$$
\mathscr{H}^{1}\left(X, \mathcal{G}_{1} \times \mathcal{G}_{2}\right) \cong \mathscr{H}^{1}\left(X, \mathcal{G}_{1}\right) \times \mathscr{H}^{1}\left(X, \mathcal{G}_{2}\right)
$$

and for which the above pairing of $\mathscr{F}_{1}$ and $\mathscr{F}_{2}$ is bi-equivariant. Then we obtain a twisted pairing, where the $\mathscr{G}_{i}$ are now gerbes for the $\mathcal{G}_{i}$ :

$$
\widetilde{P}: \mathscr{F}_{1}\left(\mathscr{G}_{1}\right) \times \mathscr{F}_{2}\left(\mathscr{G}_{2}\right) \rightarrow \mathscr{F}_{3}\left(\mathscr{G}_{1} \times \mathscr{G}_{2}\right),
$$

invoking Lemma I.1.5 (1)] Proposition I.4.2 can be recovered when $\mathcal{G}_{1}=\mathcal{G}_{2}$ by pushing forward along the product map, by Lemma I.1.5) (2)

A special case of this construction is in the relative situation of a map $f: Y \rightarrow X$, where we have a sheaf of categories $\mathscr{F}$ in the large topology on $X$ with a tensor product and a gerbe $\mathscr{G}$ on $Y$ :

Corollary I.4.3. In the above setup, we may form products $s \otimes f^{*} t$, where $s \in$ $\mathscr{F}(\mathscr{G})_{Y}$ and $t \in \mathscr{F}_{X}$.

Proof. Let $\Gamma_{f}: Y \rightarrow Y \times X$ be the graph of $f$, so $s \otimes f^{*} t=\Gamma_{f}^{*}(s \otimes t)$, where $s \otimes t$ makes sense as an object twisted by $\mathscr{G} \otimes \mathscr{G}^{0}$ on $Y \times X$, and $\Gamma_{f}^{*}\left(\mathscr{G} \otimes \mathscr{G}^{0}\right)=\mathscr{G} \otimes f^{*} \mathscr{G}^{0} \cong \mathscr{G}$.

Twisted equivariant pairings. The content of this section is unlikely to appear motivated until at least the advent of Proposition II.4.3, and probably not until Lemma IV.7.7. However, they embody an important (if obscure) principle which we might state as follows. Suppose $S$ is a type of structure (for example, grading by some group together with a graded tensor product) and suppose that it can be specified on a category, or sheaf of categories, in many a priori inequivalent waysall different, however, only in the morphisms specified by the structure (rather than the functors). Then these differences can be reproduced via twisting by trivial gerbes having structure $S$.

Let $G$ be an $X$-group and let $\mathscr{F}$ be a sheaf of categories on $G$; as usual, we denote $\operatorname{pr}_{1}, \mathrm{pr}_{2}, m: G \times_{X} G \rightarrow G$.

Definition I.4.4. A $G$-equivariant pairing on $\mathscr{F}$ is a map of stacks

$$
\bigotimes: \operatorname{pr}_{1}^{*} \mathscr{F} \times \operatorname{pr}_{2}^{*} \mathscr{F} \rightarrow m^{*} \mathscr{F}
$$

i.e., a pairing $\left(\operatorname{pr}_{1}^{*} s, \operatorname{pr}_{2}^{*} t\right) \mapsto m^{*}(s \otimes t)$ for any sections $s, t \in \mathscr{F}_{G}$. This should be associative in a manner compatible with the associativity of $G$. If $G$ is commutative, then we have a natural associated notion of commutative $G$-equivariant 
pairing in which we impose a commutativity constraint isomorphism on this pairing. Explicitly, it is an isomorphism

$$
m^{*}(s \otimes t) \cong m^{*}(t \otimes s)
$$

for every $s, t \in \mathscr{F}_{G}$, compatible with associativity in triple products.

An example of such a pairing would be, as suggested above, a $G$-graded tensor product if $G$ happens to be a discrete (abelian) group. Now let $\mathscr{G}$ be a (possibly commutative) multiplicative $\mathcal{G}$-gerbe on $G$; if $\mathscr{F}$ has an action of $\mathscr{H}^{1}(X, \mathcal{G})$ and if the above pairing is $\mathcal{G}$-bi-equivariant (in the sense of Definition I.4.1) then we have a twisting

$$
\operatorname{pr}_{1}^{*} \mathscr{F}\left(\operatorname{pr}_{1}^{*} \mathscr{G}\right) \times \operatorname{pr}_{2}^{*} \mathscr{F}\left(\operatorname{pr}_{2}^{*} \mathscr{G}\right) \rightarrow m^{*} \mathscr{F}\left(\operatorname{pr}_{1}^{*} \mathscr{G} \otimes_{X} \operatorname{pr}_{2}^{*} \mathscr{G}\right) \cong m^{*} \mathscr{F}\left(m^{*} \mathscr{G}\right)
$$

Thus, the stack $\mathscr{F}(\mathscr{G})$ has a (commutative) multiplicative $G$-equivariant pairing as well. If $\mathscr{G}$ is trivial as a multiplicative gerbe, then its commutative multiplicative structure consists of the additional data of a single isomorphism which is identified with a section of $\mathcal{G}(G \times G)$.

Proposition I.4.5. The twisting of a commutative $G$-equivariant pairing $\otimes$ on $\mathscr{F}$ by a commutative trivially multiplicative gerbe $\mathscr{G}$ is equivalent to multiplying the commutativity constraint of $\otimes$ by the above section of $\mathcal{G}(G \times G)$.

\section{I.5. Construction of Gerbes From ALGEBraic GeOMEtRy}

Our main source of concretely-specified gerbes will be those given as "groupvalued Cartier divisors". Let $Y$ be a scheme over $\mathbb{C}$; we say that a closed subspace $Z \subset Y$ has local tubular neighborhoods if it admits a covering by open sets $V$ in $Y$ such that each $V$ can be written as the product $C \times \mathbb{C}$, where $C$ is contractible, with $Z \cap V=C \times\{0\}$. This notion clearly requires the "analytic" topology, which is why we restrict to schemes over $\mathbb{C}$. For brevity, we will refer to $Z$ as a "smooth divisor".

\section{Order of a "meromorphic" map of gerbes.}

Lemma I.5.1. Let $D \subset Y$ be a connected, smooth divisor and let $U$ be its complement. Let $A$ be an abelian group (written multiplicatively) also denoting the constant sheaf with group $A$, let $\mathscr{G}$ and $\mathscr{H}$ be $A$-gerbes on $Y$, and let $f:\left.\left.\mathscr{G}\right|_{U} \rightarrow \mathscr{H}\right|_{U}$ be a map of gerbes; we refer to this as a meromorphic map from $\mathscr{G}$ to $\mathscr{H}$. Then $f$ has an associated element $\operatorname{ord}_{D}(f) \in A$, the $A$-valued order of $f$ about $D$, with the following properties:

(1) $\operatorname{ord}_{D}(f)$ is local on $D$ in that if $Z \subset Y$ is any subspace transverse to $D$ and $C$ is a component of $Z \cap D$, then $\operatorname{ord}_{D}(f)=\operatorname{ord}_{C}\left(\left.f\right|_{Z \cap U}\right)$.

(2) If $g:\left.\left.\mathscr{H}\right|_{U} \rightarrow \mathscr{K}\right|_{U}$, then $\operatorname{ord}_{D}(g \circ f)=\operatorname{ord}_{D}(g) \operatorname{ord}_{D}(f)$, and if $f^{\prime}:\left.\mathscr{G}^{\prime}\right|_{U} \rightarrow$ $\left.\mathscr{H}^{\prime}\right|_{U}$, then $\operatorname{ord}_{D}\left(f \otimes f^{\prime}\right)=\operatorname{ord}_{D}(f) \operatorname{ord}_{D}\left(f^{\prime}\right)$.

(3) For any $a \in A$, there exists a unique gerbe $\mathcal{O}(D)^{\log a}$ and trivialization $\mathcal{G}^{0} \rightarrow \mathcal{O}(D)^{\log a}$ with order a; for any map $f$ with $\operatorname{ord}_{D}(f)=a$, the induced map $\left.\left.\mathscr{F}\right|_{U} \otimes \mathcal{O}(D)^{\log a} \rightarrow \mathscr{H}\right|_{U}$ with order 1 extends to an equivalence $\mathscr{H} \cong$ $\mathscr{F} \otimes \mathcal{O}(D)^{\log a}$.

The notation $\mathcal{O}(D)^{\log a}$ is chosen to match point (2), since we then have (formally) $\mathcal{O}(D)^{\log a} \otimes \mathcal{O}(D)^{\log b}=\mathcal{O}(D)^{\log a+\log b}=\mathcal{O}(D)^{\log (a b)}$, as claimed there. 
Proof. Let $V=C \times \mathbb{C}$ be a tubular neighborhood on $D$, so $D \cap V=C \times\{0\}$ and $U \cap V=C \times \mathbb{C}^{*}$, and let $t:\left.\left.\mathscr{G}^{0}\right|_{V} \rightarrow \mathscr{G}\right|_{V}, u:\left.\left.\mathscr{G}^{0}\right|_{V} \rightarrow \mathscr{H}\right|_{V}$ be trivializations. Since $V$ is contractible, in fact there are no nontrivial automorphisms ( $A$-torsors) of $\left.\mathscr{G}^{0}\right|_{V}$, so this choice is canonical and, most notably, compatible with refinement of $V$. We have two maps $u t^{-1}, f:\left.\left.\mathscr{G}\right|_{U \cap V} \rightarrow \mathscr{H}\right|_{U \cap V}$ which, thus, differ by the action of some $A$-torsor $\mathcal{T}$ on $U \cap V$. Since $C$ is contractible, this is the same as giving just an $A$-torsor on $\mathbb{C}^{*}$, and hence an element of $A$ which we define to be $\operatorname{ord}_{D}(f)$. Since $t$ and $u$ are compatible with refinement of $V$, this number is well-defined and locally constant on $D$, hence constant since $D$ is connected.

To show (1), we note that $\operatorname{ord}_{D}(f)$ depends only on the transverse portion of $V$. Point (2) is clear, since the tensor product of maps is the same as the tensor product of their corresponding torsors $\mathcal{T}$ (from the previous paragraph), and likewise for composition. For point (3), it is immediate from the definition that any map of order 1 extends uniquely, since this is true of the torsors $\mathcal{T}$. We must construct $\mathcal{O}(D)^{\log a}$, for which there are several options.

We give the most direct here. Given any tubular neighborhood $V$, let $\left.\mathcal{O}(D)^{\log a}\right|_{V}$ be the trivial gerbe and let its trivialization $t_{V}$ on $U \cap V$ be given by the torsor $\mathcal{T}_{V}$ on $\mathbb{C}^{*}$ with order $a$ about 0 , extended to a torsor on $C \times \mathbb{C}^{*}$. For two neighborhoods $V_{1}$ and $V_{2}$, on $\left(V_{1} \cap V_{2}\right) \cap U$, the composition $t_{V_{2}} t_{V_{1}}^{-1}$ has order 1 about $D$ and therefore extends to an equivalence $\phi$ of $\left.\mathcal{O}(D)^{\log a}\right|_{V_{1}}$ with $\left.\mathcal{O}(D)^{\log a}\right|_{V_{2}}$ on $V_{1} \cap V_{2}$. By definition, the $\phi$ 's have a cocycle constraint (a 2-isomorphism in $\mathbf{H}^{2}\left(V_{1} \cap V_{2} \cap\right.$ $\left.V_{3}, A\right)$ ) on triple intersections which themselves form a 2-cocycle, so we may glue the $\left.\mathcal{O}(D)^{\log a}\right|_{V}$ over all $V$ (including those which do not intersect $U$ at all) to get $\mathcal{O}(D)^{\log a}$ on $Y$ and a trivialization $t$ on $U$ with order $a$, by definition.

There is a generalization of Lemma I.5.1 to reducible divisors, although the proof does not generalize since such divisors do not have local tubular neighborhoods at the crossings.

Lemma I.5.2. Let $D=\bigcup_{i} D_{i}$ be a union (not necessarily disjoint) of connected, smooth divisors, $f$ as before. Then $f$ has orders $a_{i}$ about the $D_{i}$ inducing an equivalence $\mathscr{H} \cong \mathscr{G} \otimes \bigotimes_{i} \mathcal{O}\left(D_{i}\right)^{\log a_{i}}$.

Proof. We proceed by induction on the number $n$ of components of $D$. If $n=1$, then this is just Lemma I.5.1. In general, let $Y=X \backslash D_{n}$, which has the divisor $D^{\prime}=\bigcup_{i<n} D_{i}$, and so by induction, the lemma holds on $Y$. Thus, we have

$$
\left.\left.\mathscr{H}\right|_{Y} \cong \mathscr{G}\right|_{Y} \otimes \bigotimes_{i<n} \mathcal{O}_{Y}\left(D_{i} \backslash D_{n}\right)^{\log a_{i}}
$$

for some $a_{i}$. We write $\mathcal{O}_{Y}$ rather than $\mathcal{O}$ to emphasize that the latter gerbes are specific to $Y$; however, we have $\left.\mathcal{O}\left(D_{i}\right)^{\log a_{i}}\right|_{Y} \cong \mathcal{O}_{Y}\left(D_{i} \backslash D_{n}\right)^{\log a_{i}}$. Thus, the above equation can be written

$$
\left.\left.\mathscr{H}\right|_{Y} \cong\left(\mathscr{G} \otimes \bigotimes_{i<n} \mathcal{O}\left(D_{i}\right)^{\log a_{i}}\right)\right|_{Y}
$$

and so again by Lemma I.5.1, we deduce the existence of some order $a_{n}$ and the lemma follows.

It should be noted that the success of this lemma is due to the fact that $a_{i}$ is constant along $D_{i}$, and so extends from the complement of several points. In the context of a possible theory of higher gerbes, the analogue of Lemma I.5.1 should 
be expected to hold for "orders" valued in other higher gerbes (but not as high) but the above lemma will fail since these orders will not necessarily extend.

Geometric construction of the twisting gerbe. An alternative and much nicer construction of $\mathcal{O}(D)^{\log a}$ can be extracted from the line bundle $\mathcal{O}(D)$, when $D$ is a Cartier divisor. Let $L$ be the total space of $\mathcal{O}(D)$, so that each fiber of $L$ over a point of $Y$ is isomorphic to $\mathbf{G}_{\mathbf{m}}$ or, topologically, to $\mathbb{C}^{*}$. For each $V \subset Y$, let $\mathcal{O}(D)_{V}^{\log a}$ be the category of $A$-torsors on $\left.L\right|_{V}$ such that, on each fiber, they have monodromy a. More precisely, let $\mathcal{L}_{a}$ be the local system on $\mathbf{G}_{\mathbf{m}}$ with fiber $A$ and monodromy $a$, which is a multiplicative, or "character", sheaf in the sense of Definition I.3.2, if $m: \mathbf{G}_{\mathbf{m}} \times \mathbf{G}_{\mathbf{m}} \rightarrow \mathbf{G}_{\mathbf{m}}$, we have

$$
m^{*}\left(\mathcal{L}_{a}\right) \cong \mathcal{L}_{a} \otimes \mathcal{L}_{a},
$$

compatibly with the group structure of $m$. We consider $L$ as a $\mathbf{G}_{\mathbf{m}}$-torsor with action map $c: \mathbf{G}_{\mathbf{m}} \times L \rightarrow L$ and define $\mathcal{O}(D)_{V}^{\log a}$ to be the category of twisted equivariant $A$-torsors $\mathcal{T}$ on $\left.L\right|_{V}$ : as in Definition I.3.2, we are given

$$
c^{*} \mathcal{T} \cong \mathcal{L}_{a} \otimes \mathcal{T}
$$

compatibly with the unit section of $\mathbf{G}_{\mathbf{m}} \times L$ and the multiplicativity of $\mathcal{L}_{a}$.

Proposition I.5.3. Suppose D has multiplicity 1 as a connected Cartier divisor. Then the above prescription defines an A-gerbe "O $(D))^{\log a "}$ with a trivialization on $U$ of order $a$.

Proof. If $\mathcal{O}(D)$ is trivialized on $V$, then we have $\left.L\right|_{V} \cong \mathbf{G}_{\mathbf{m}} \times V$; then for any $A$ torsor $\mathcal{T}$ on $V$, we can take $\widetilde{\mathcal{T}}=\mathcal{L}_{a} \otimes \mathcal{T}$, where $\mathcal{L}_{a}$ is the unique $A$-torsor on $\mathbf{G}_{\mathbf{m}}$ with order $a$. Thus, "OO(D) ${ }^{\log a}$ " has local sections on $Y$. It is obvious that we can glue sections on $Y$, since we can glue them as $A$-torsors on $L$, so "OOO$(D)$ log $a$ " is a sheaf of categories. The action of $\mathscr{H}^{1}(Y, A)$ is given by: for every $A$-torsor $\mathcal{S}$ on $V$ and every $a$-order $A$-torsor $\mathcal{T}$ on $\left.L\right|_{V}$, we set $\mathcal{S} \cdot \mathcal{T}=\operatorname{pr}_{Y}^{*}(\mathcal{S}) \otimes \mathcal{T}$, which is still $a$-twisted since the pullback is plain equivariant. If $\mathcal{T}_{1}, \mathcal{T}_{2}$ are two sections of "OOO $(D)_{V}^{\log a}$ ", then $\mathcal{T}_{2} \otimes \mathcal{T}_{1}^{-1}$ is $\mathbf{G}_{\mathbf{m}}$-equivariant and, hence, is of the form $\operatorname{pr}_{Y}^{*}(\mathcal{S})$ for some $\mathcal{S}$ on $V$; then $\mathcal{T}_{2}=\mathcal{S} \cdot \mathcal{T}_{1}$, and this identification is obviously free, so "OO $(D)^{\log a "}$ is a gerbe.

We identify $\mathcal{O}(D)$ with the sheaf of meromorphic functions on $Y$ with poles only at $D$ and of order at most 1 ; thus, on any open set $V$ on which $D$ is given by the equation $z=0, \mathcal{O}(D)$ admits a trivialization $t: \mathcal{O} \rightarrow \mathcal{O}(D)$ sending 1 to $z^{-1}$. Let $f$ be the trivialization on $U$ sending 1 to 1 . These trivializations give rise to two isomorphisms $\tau, \phi: \mathbf{G}_{\mathbf{m}} \times\left. V \rightarrow L\right|_{V}$; here, $\tau^{-1} \phi=(z$, id $)$ is multiplication of the first coordinate by the parameter $z$ on $\mathbf{G}_{\mathbf{m}}$. Let $\tilde{\mathcal{T}}=\mathcal{L}_{a} \otimes \mathcal{T}$ be a section of "OOO $(D)^{\log a} "$ relative to the trivialization $\tau$; then relative to $\phi, \widetilde{\mathcal{T}}=z^{*} \mathcal{L}_{a} \otimes \mathcal{T}$. But $z^{*} \mathcal{L}_{a}=\mathcal{L}_{a} \otimes \mathcal{L}_{a}$, so if we call the induced trivializations of " $\mathcal{O}(D)^{\log a}$ " $T$ and $F$, then $F$ differs from $T$ by the action of $\mathcal{L}_{a}$ and hence $F$ has order $a$ (and is defined on all of $U)$, as desired.

Excluding the trivialization, this construction also furnishes a gerbe $\mathcal{L}^{\log a}$ for every line bundle $\mathcal{L}$ on $Y$ and every $a \in A$, which will be our main (even only) source of interesting gerbes. We note the following property, which is obvious from the definition and the computation in the preceding proof: 
Proposition I.5.4. The construction $\mathcal{L} \mapsto \mathcal{L}^{\log a}$ is a 2 -functor from line bundles to A-gerbes. (This means that given maps $\mathcal{L}_{1} \rightarrow \mathcal{L}_{2} \rightarrow \mathcal{L}_{3}$ of line bundles, the map $\mathcal{L}_{1}^{\log a} \rightarrow \mathcal{L}_{3}^{\log a}$ is given an isomorphism with the composition of the other two, and this isomorphism is compatible with associativity.) We have $\left(\mathcal{L}^{n}\right)^{\log a} \cong \mathcal{L}^{\log a^{n}}$ and, for any map $f: Z \rightarrow Y$, we have $\left(f^{*} \mathcal{L}\right)^{\log a} \cong f^{*} \mathcal{L}^{\log a}$. In fact, we have $(\mathcal{L} \otimes \mathcal{M})^{\log a} \cong \mathcal{L}^{\log a} \otimes \mathcal{M}^{\log a}$ for any line bundles $\mathcal{L}, \mathcal{M}$.

Order and equivariant gerbes. We note the corollary of the proof of Lemma I.5.1

Corollary I.5.5. Let $c: \tilde{Y} \rightarrow Y$ be a map which is a d-fold covering map away from $\widetilde{D}=c^{-1}(D)$, and let $\widetilde{f}:\left.\left.c^{*} \mathscr{G}\right|_{\widetilde{U}} \rightarrow c^{*} \mathscr{H}\right|_{\widetilde{U}}$ be the pullback of $f$. If $\left.c\right|_{\widetilde{D}}$ is an isomorphism, then $\operatorname{ord}_{\widetilde{D}}(\widetilde{f})=\operatorname{ord}_{D}(f)^{d}$; if c extends to a covering map of $Y$, then for every component $E$ of $\widetilde{D}$, we have $\operatorname{ord}_{E}(\widetilde{f})=\operatorname{ord}_{D}(f)$.

Proof. These facts are true of $A$-torsors on $\mathbb{C}^{*}$.

This corollary is descriptive, in that the gerbes on $Y$ must exist. Morally, such gerbes are equivalent to gerbes on $\tilde{Y}$ with suitable descent data, and we will need the following simple special case. To state it, we need to make a definition that will figure prominently later.

Definition I.5.6. Let $S_{2}$ act on a space $Y$, and let $s$ be the nonidentity element. The structure of $S_{2}$-equivariance for an $A$-gerbe $\mathscr{G}$ on $Y$, for an action of $S_{2}$ on $Y$, is the the data of an equivalence $\phi: \mathscr{G} \rightarrow s^{*} \mathscr{G}$ and a 2 -isomorphism $i: \phi \rightarrow s^{*} \phi^{-1}$ such that $s^{*} i \circ i=$ id as a 2-automorphism of $\phi$.

Let $f: \mathscr{G} \rightarrow \mathscr{H}$ be a map of $S_{2}$-equivariant gerbes $(\mathscr{H}$ with structure $\psi, j$ ). The structure of $S_{2}$-equivariance on it is a 2-isomorphism $c: \psi \circ f \rightarrow s^{*} f \circ \phi$ of maps $\mathscr{G} \rightarrow s^{*} \mathscr{H}$ such that in the diagram below,

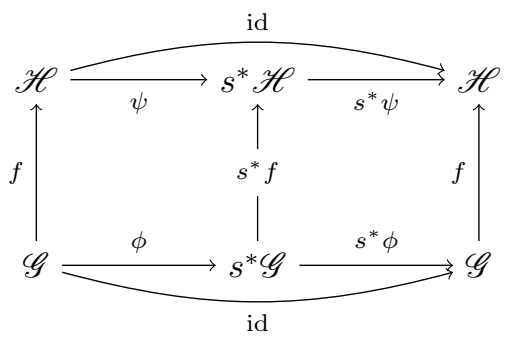

the 2-automorphism of $f$ obtained by applying $j, c, s^{*} c$, and $i$ to go from the top-most path to the bottom-most path, is the identity.

If $S_{2}$ acts trivially on $Y$, then we define the trivial $S_{2}$-equivariance structure on $\mathscr{G}$ to be that with $\phi=\mathrm{id}$ and $i=\mathrm{id}$; a trivialization of any $S_{2}$-equivariance structure is an equivalence with this one.

Lemma I.5.7. Let $s$ be an involution on $Y$ with fixed-point set a connected smooth divisor $D$ with and complement $U$, and let $\mathscr{G}$ be an $S_{2}$-equivariant gerbe on $Y$ together with a trivialization of this structure on $\mathscr{G}_{\left.\right|_{D}}$. Let $\mathscr{H}$ be another $S_{2}$ equivariant gerbe and $f:\left.\left.\mathscr{G}\right|_{U} \rightarrow \mathscr{H}\right|_{U}$ an $S_{2}$-equivariant meromorphic map between them with $\operatorname{ord}_{D}(f)=a$. Then the choice of an element $b \in A$ with $b^{2}=a$ is equivalent to the choice of a trivialization of the $S_{2}$-equivariance on $\left.\mathscr{H}\right|_{D}$. 
The statement of this lemma is motivated by the observation that if $Y / S_{2}$ existed and $\mathscr{G}$ descended, then it would have an $S_{2}$-equivariance structure, and since $Y \rightarrow Y / S_{2}$ would be an isomorphism on $D$, this structure would be trivial there. Likewise, an $S_{2}$-equivariant gerbe $\mathscr{H}$ would descend only if the same held for it, and then the $S_{2}$-equivariant meromorphic map $f$ would descend as well and we could apply Corollary I.5.5 to get the order claimed.

Proof of Lemma. Suppose we had such a trivialization for $\left.\mathscr{H}\right|_{D}$. Since order is defined locally on $D$, we assume that $Y=\mathbb{C}$ and $s$ is rotation by $\pi$, fixing the origin $D=\{0\}$. Then we may also assume that $\mathscr{G}$ and $\mathscr{H}$ are the trivial gerbes, so that $\phi$ and $\psi$ are identified with $A$-torsors on $\mathbb{C}$, hence also trivial, and so $i$ and $j$ are identified with square roots of 1 in $A$; the trivializations of the $S_{2}$-structures over $D$ give $i=j=1$. Likewise, $f$ is identified (by definition) with the $A$-torsor $\mathcal{L}_{a}$ having monodromy $a$, so $c$ is an isomorphism of $\mathcal{L}_{a}$ with $s^{*} \mathcal{L}_{a}$ and the commutativity of diagram (I.8) becomes the identity $s^{*} c \circ c=$ id.

We claim that such an isomorphism $c$ gives a square root of $a$. Indeed, consider the germ of $c$ acting as $\left(\mathcal{L}_{a}\right)_{1} \rightarrow\left(s^{*} \mathcal{L}_{a}\right)_{1}=\left(\mathcal{L}_{a}\right)_{-1}$. These two stalks are already identified by means of a counterclockwise path $\gamma$ from 1 to -1 in $\mathbb{C}$, so fixing this identification, $c$ is just some element $b \in A$. Likewise, $s^{*} c$ is multiplication by $b$ when compared to the isomorphism $\left(\mathcal{L}_{a}\right)_{-1} \cong\left(\mathcal{L}_{a}\right)_{1}$ induced by $s(\gamma)$. Thus, $s^{*} c \circ c=b^{2}$ when compared to $s(\gamma) \circ \gamma$, whose associated automorphism of $\left(\mathcal{L}_{a}\right)_{1}$ is, by definition, multiplication by $a$. Since $s^{*} c \circ c=$ id, we must have $b^{2}=a$.

Conversely, suppose we have the square root $b$, and let $\mathcal{O}(D)^{\log a}$ have the $S_{2^{-}}$ equivariance structure extending that of the trivial gerbe on $U$, using Lemma I.5.1](3) Using $b$ and the logic from the previous paragraph, we extract a trivialization of the $S_{2}$-equivariance structure of $\mathcal{O}(D)^{\log a}$ on $D$. Then the isomorphism $\mathscr{G} \otimes$ $\mathcal{O}(D)^{\log a} \rightarrow \mathscr{H}$ induced by $f$ is in fact an isomorphism of $S_{2}$-equivariant gerbes; in particular, the trivialization of that structure on the left-hand side over $D$ induces a trivialization of the equivariance structure on $\left.\mathscr{H}\right|_{D}$, as desired.

\section{Part II. Symmetric factorizable gerbes and their classification by quadratic forms}

This part is devoted to investigating properties of gerbes on a certain kind of space, one with the property of factorizability. The principal goal is to show that the natural data on a gerbe which makes it compatible with this structure renders the category of all such gerbes easily described by simple algebraic objects. In this part, we restrict our attention to those properties of factorizable gerbes which can be related directly to the topology of the underlying factorizable space, while in Part III, we will add additional data and derive additional structure.

\section{II.1. THE AFFINE GRASSMANNIAN AND FACTORIZABILITY}

We will use the following notation throughout the rest of this part or even the rest of the paper. The scheme $X$ will be a smooth complex algebraic curve (not necessarily complete). For any scheme $S$ and any $S$-point $\vec{x} \in X^{n}(S)$, let $\bar{x} \subset X_{S}=$ $S \times X$ be the scheme-theoretic union of the graphs of its coordinates, a union of Cartier divisors since $X$ is smooth; we will not need the scheme structure, however. 
Definition II.1.1. Let $G$ be a complex algebraic group. Taken over all integers $n$, the following functors from schemes to sets constitute the relative or factorizable or Beilinson-Drinfeld affine grassmannian:

$$
\operatorname{Gr}_{G, X^{n}}(S)=\left\{(\vec{x}, \mathcal{T}, \phi) \mid \begin{array}{l}
\vec{x} \in X^{n}(S), \mathcal{T} \text { is a } G \text {-torsor on } X_{S}, \\
\phi \text { is a trivialization of } \mathcal{T} \text { on } X_{S} \backslash \bar{x}
\end{array}\right\} .
$$

Only in this definition, by $G$-torsor, we mean local triviality in the étale topology. By definition, $\operatorname{Gr}_{G, X^{n}}$ is naturally a functor on schemes over $X^{n}$. We will write $\mathrm{Gr}_{n}$ when neither $G$ nor $X$ need be mentioned.

As mere presheaves of sets, these functors satisfy the following well-known theorem (see e.g. BD, Theorem 4.5.1]):

Proposition. The functors $\mathrm{Gr}_{G, X^{n}}$ are representable by ind-schemes; they are ind-projective if and only if $G$ is reductive.

We denote, as is usual, the ordinary affine grassmannian $G((t)) / G\left[[t]\right.$ by $\mathrm{Gr}_{G}$; it is obtained as the fiber of $\mathrm{Gr}_{G, X}$ over any point of $X$ (but not canonically). In fact, it is easy to see that $\operatorname{Gr}_{G, X^{n}}$ is a bundle over the complement of all the diagonals in $X^{n}$, where the fiber is $\mathrm{Gr}_{G}^{n}$; this will be a special case of Proposition II.1.3.

We will return to the grassmannian of a general group later. Now, let $T$ be a complex algebraic torus; since $T$ is a commutative group, its torsors admit a commutative group operation which we denote by the symbol $\otimes$; tensor product of torsors makes $\mathrm{Gr}_{T, X^{n}}$ a group functor over $X^{n}$. In fact, we have a pairing over $X^{n+m}$ :

$$
\mathrm{Gr}_{T, X^{n}} \times \mathrm{Gr}_{T, X^{m}} \rightarrow \mathrm{Gr}_{T, X^{n+m}} .
$$

We can give a more detailed description of the grassmannian of a torus. We denote the weight and coweight lattices of $T$, respectively, by $\Lambda^{T}=X^{*}(T)$ and $\Lambda_{T}=X_{*}(T)$; we use the notation $\langle\bullet, \bullet\rangle: \Lambda^{T} \otimes \Lambda_{T} \rightarrow \mathbb{Z}$ for the natural pairing. In the second part of the following proposition, we refer to certain diagonals in $X^{n}$ corresponding to partitions $p$ of $\{1, \ldots, n\}$ into subsets $p_{i}$ :

$$
\Delta_{p}^{n}=\bigcap_{i}\left\{x_{k}=x_{l} \mid k, l \in p_{i}\right\} .
$$

We will also sometimes write $\Delta_{n_{1}, n_{2}, \ldots}^{n}$, in which case we mean that $p$ should have parts $\left\{n_{1}, n_{2}, \ldots\right\}$ and singletons consisting of all unmentioned indices. That is, $\Delta_{1,2}^{3}=\left\{x_{1}=x_{2}\right\}$, and not $\left\{x_{2}=x_{3}\right\}$, as might be imagined if the 2 is taken to mean "group the last two numbers of $\{1,2,3\}$ ".

Proposition II.1.2. The complex points $\operatorname{Gr}_{T, X^{n}}(\mathbb{C})$ have the following structure as ind-complex varieties:

(1) For $\lambda \in \Lambda_{T}$ and $x \in X(\mathbb{C})$, let $\mathcal{O}(\lambda x)$ be the $T$-torsor on $X$ together with a trivialization $\phi_{\lambda, x}$ on $X \backslash\{x\}$ characterized by the following property: for any $\mu \in \Lambda^{T}=\operatorname{Hom}\left(T, \mathbf{G}_{\mathbf{m}}\right)$, the line bundle ${ }^{1} \mu \mathcal{O}(\lambda x)$ together with its trivialization ${ }^{1} \mu\left(\phi_{\lambda, x}\right)$ is isomorphic to $\mathcal{O}(\langle\mu, \lambda\rangle x)$. The map $X(\mathbb{C}) \times \Lambda_{T} \rightarrow$ $\operatorname{Gr}_{T, X}(\mathbb{C})$ given by

$$
(x, \lambda) \mapsto\left(x, \mathcal{O}(\lambda x), \phi_{\lambda, x}\right)
$$

is an isomorphism of groups over $X(\mathbb{C})$. 
(2) The multiplication (II.1) identifies the irreducible components of $\operatorname{Gr}_{T, X^{n}}(\mathbb{C})$ with the products of those of $\operatorname{Gr}_{T, X}(\mathbb{C})$; they are thus indexed by $\left(\Lambda_{T}\right)^{n}$ and isomorphic to $X^{n}(\mathbb{C})$. Let $p$ be a partition of $\{1, \ldots, n\}$ into parts $p_{j}=\left\{i_{1}, \ldots, i_{n_{j}}\right\}\left(1 \leq i_{k} \leq n\right)$, and let $\Delta_{p}^{n}$ be the corresponding diagonal of $X^{n}$. Then the intersection of two components $\mathrm{Gr}_{n}^{\lambda_{1}, \ldots, \lambda_{n}}, \mathrm{Gr}_{n}^{\mu_{1}, \ldots, \mu_{n}}$ is $\Delta_{p}^{n}$ (identifying $X^{n}$ with both components) if and only if for each $j$, we have $\lambda_{i_{1}}+\cdots+\lambda_{i_{n_{j}}}=\mu_{i_{1}}+\cdots+\mu_{i_{n_{j}}}$.

We will only ever use the topological structure of the grassmannians, so we will not mention the $\mathbb{C}$-points subsequently. The structure exhibited in the above proposition is formalized the the following definition:

Proposition II.1.3. The $\mathrm{Gr}_{n}$ are factorizable:

(1) For $n, m \in \mathbb{N}$, let $p$ be a partition of $[1, n]$ into $m$ parts and $\Delta_{p}^{n}$ be the corresponding copy of $X^{m}$ inside $X^{n}$. Then there are isomorphisms

$$
\left.\mathrm{Gr}_{n}\right|_{\Delta_{p}^{n}} \cong \mathrm{Gr}_{m}
$$

which are compatible with refinement of the partition $p$;

(2) Let $p$ be a partition as above and suppose its parts $p_{i}$ have sizes $n_{i}$; let $X_{p}^{n}$ be the open subset of $X^{n}$ consisting of coordinates $\vec{x}=\left(x_{1}, \ldots, x_{n}\right)$ such that if $x_{i}=x_{j}$, then $i, j$ are in the same part of $p$. Then there are isomorphisms

$$
\left.\left.\mathrm{Gr}_{n}\right|_{X_{p}^{n}} \cong\left(\prod \mathrm{Gr}_{n_{i}}\right)\right|_{X_{p}^{n}}
$$

compatible with refinement of the partition $p$ (together with, of course, further restrictions to finer $X_{p}^{n}$ 's). Furthermore, these isomorphisms are compatible with those above when restricting both to some diagonal, and away from others, in either order.

(3) For any $n$, we have an equivariance structure for the action of the symmetric group $S_{n}$ on $X^{n}$ which is compatible with both of the above classes of isomorphisms.

The details of the second point will be relevant later, so we describe them fully.

Proof. Points (1) and (3) are obvious, so we consider only (2) Suppose that we have $m$ groups $p_{i}$ of coordinates' indices such that their graphs are disjoint: $\Gamma\left(x_{k}\right) \cap$ $\Gamma\left(x_{l}\right)=\varnothing$ for $x_{k} \in p_{i}$ and $x_{l} \in p_{j}$ with $i \neq j$. Let the coordinate groups be denoted $\vec{x}_{i}$, let $\bar{x}_{i}$ be the union of the graphs of the coordinates in each group, let $U_{i}$ be their complements in $X_{S}$, and let $V_{i}=\bigcap_{j \neq i} U_{j}$. We establish mutually inverse isomorphisms of $\mathrm{Gr}_{n} \mid X_{p}^{n}$ with $\prod_{i} \mathrm{Gr}_{n_{i}}$ as follows:

- For $\left.(\vec{x}, \mathcal{T}, \phi) \in \operatorname{Gr}_{n}\right|_{X_{p}^{n}}$, let $\mathcal{T}_{i}$ be the $G$-torsor on $X_{S}$ with $\left.\mathcal{T}_{i}\right|_{U_{i}}=\mathcal{T}^{0}$ the trivial torsor and $\left.\left.\mathcal{T}_{i}\right|_{V_{i}} \cong \mathcal{T}\right|_{V_{i}}$, with the isomorphism on $U_{i} \cap V_{i}=$ $X_{S} \backslash \bar{x}$ given by $\phi$; thus, there is a natural trivialization $\phi_{i}$ of $\left.\mathcal{T}_{i}\right|_{U_{i}}$. Then $\left(\vec{x}_{i}, \mathcal{T}_{i}, \phi_{i}\right) \in \mathrm{Gr}_{n_{i}}(S)$, as desired.

- Given points $\left(\vec{x}_{i}, \mathcal{T}_{i}, \phi_{i}\right) \in \operatorname{Gr}_{n_{i}}(S)$ with all the $\vec{x}_{i}$ disjoint from one another, let $\mathcal{T}$ be the $G$-torsor with $\left.\left.\mathcal{T}\right|_{V_{i}} \cong \mathcal{T}_{i}\right|_{V_{i}}$ for each $i$, where on $V_{i} \cap V_{j}=\bigcap_{k} U_{k}$, the isomorphisms are given by $\phi_{i} \phi_{j}^{-1}$, which are clearly compatible on triple intersections. There is a natural trivialization $\phi$ of $\mathcal{T}$ on $\bigcap V_{i}=\bigcap U_{i}=X_{p}^{n}$ induced by any of the $\phi_{i}$ 's. Taking $\vec{x}$ to be the union of the $\vec{x}_{i}$, we have $(\vec{x}, \mathcal{T}, \phi) \in \mathrm{Gr}_{n} \mid X_{p}^{n}(S)$, as desired. 
It is easy to see that these constructions are inverse to one another.

When $G=T$ is a torus, the gluing of point (2) can be realized by taking the tensor product of $T$-torsors as well. That is, given points $\left(\vec{x}_{i}, \mathcal{T}_{i}, \phi_{i}\right) \in \operatorname{Gr}_{m_{i}}(S)$, we can simply define $\mathcal{T}=\bigotimes_{i} \mathcal{T}_{i}$ and, on $X_{S} \backslash \bar{x}$, trivialize it via $\phi=\left.\bigotimes_{i} \phi_{i}\right|_{X_{S} \backslash \bar{x}}$. This shows that the description of Proposition II.1.2 is indeed the same as the factorizable structure just given.

An example of point (2) is when $\Lambda_{T}=\mathbb{Z}$ and $n=3$; then we can say that $\mathrm{Gr}_{3}^{0,4,-1}$ and $\mathrm{Gr}_{3}^{2,2,-1}$ intersect in the divisor $\Delta_{1,2}^{3}$ where $x_{1}=x_{2}$, while $\mathrm{Gr}_{3}^{1,1,1}$ intersects either of them only in the diagonal $\Delta_{1,2,3}^{3}$ where $x_{1}=x_{2}=x_{3}$. To illustrate the factorizable structure, we generalize this to say that the $\mathrm{Gr}_{3}^{a, b, c}$ are divided into groups, indexed by $(a+b, c)$, which intersect over $\Delta_{1,2}^{3}$; denote the irreducible components of the fiber $\left.\mathrm{Gr}_{3}\right|_{\Delta_{1,2}^{3}}$ by $C^{d, e}$. If we identify $\Delta_{1,2}^{3} \cong X^{2}$ in the obvious way, then $\Delta_{1,2}^{3} \cap \Delta_{1,2,3}^{3} \cong \Delta$ is the diagonal in $X^{2}$, and the $C^{d, e}$ are divided into groups, indexed by $d+e$, which intersect over $\Delta$. This is exactly the pattern of intersections of the components $\mathrm{Gr}_{2}^{d, e}$, which are furthermore isomorphic to $C^{d, e}$. We dwell on this point because it will become important later.

\section{II.2. GERBES AND FACTORIZABILITY}

In this section, we make the main definition, that of a symmetric factorizable gerbe for an abelian group $A$ on the $\mathrm{Gr}_{n}$. Our goal is to associate with every such gerbe $\mathscr{G}=\left\{\mathscr{G}_{n}\right\}$ a quadratic form $Q=Q(\mathscr{G})$ on the coweight lattice $\Lambda_{T}$, where $Q: \Lambda_{T} \rightarrow A$ (in particular, when $A$ is the multiplicative group of a field). We will construct specific examples of such gerbes and show how most such forms (in particular, the Killing form) can be obtained explicitly.

Here is the main definition of this work.

Definition II.2.1. Let $A$ be a multiplicative abelian group. A symmetric factorizable gerbe over $A$, or sf $A$-gerbe, is a collection of $A$-gerbes $\mathscr{G}_{n}$ on $\mathrm{Gr}_{n}$ with the following properties reminiscent of Proposition II.1.3

(1) For a partition $p$ of $n$ into $m$ parts, we have equivalences

$$
\left.\mathscr{G}_{n}\right|_{\Delta_{p}^{n}} \cong \mathscr{G}_{m}
$$

these come with compatibility 2-isomorphisms for refinements of partitions which are themselves compatible with further refinement.

(2) If $p$ has parts of size $n_{i}$, then we have equivalences

$$
\left.\left.\mathscr{G}_{n}\right|_{X_{p}^{n}} \cong\left(\bigotimes_{i} \mathscr{G}_{n_{i}}\right)\right|_{X_{p}^{n}}
$$

which also come with compatibility 2 -isomorphisms which are suitably compatible with refinement.

(3) $\mathscr{G}_{n}$ has a structure of $S_{n}$-equivariance which is compatible with both of the above equivalences and their compatibilities.

The vague restrictions imposed above can be made precise as follows: let $p$ be a partition of $\{1, \ldots, n\}$ into $m$ parts, let $q$ be a refinement of $p$ into $l$ parts, and let $r$ be a refinement of $q$ into $k$ parts. Then for (1), we are given the above equivalences (1-morphisms) together with 2-isomorphisms between the following 1-morphisms:

$$
\left.\left.\left.\mathscr{G}_{n}\right|_{\Delta_{p}^{n}}\right|_{\Delta_{q}^{m}} \cong \mathscr{G}_{m}\right|_{\Delta_{q}^{m}} \cong \mathscr{G}_{l},\left.\quad \mathscr{G}_{n}\right|_{\Delta_{q}^{n}} \cong \mathscr{G}_{l},
$$


and likewise for restriction through $q$ and then $r$. Furthermore, the equivalence

$$
\left.\mathscr{G}_{n}\right|_{\Delta_{r}^{n}} \cong \mathscr{G}_{k}
$$

can be realized in four ways: (1) directly, considering $r$ as a partition of $\{1, \ldots, n\}$; (2) passing first through $p$ and then considering $r$ as a refinement of it; (3) passing first through $q$ and then considering $r$ as a refinement of it; (4) passing through $p$, $q$, and $r$ in order. Let these numbers denote the resulting 1-morphism obtained by composition of the maps described, so that we have a diagram of 2 -isomorphisms

(2)

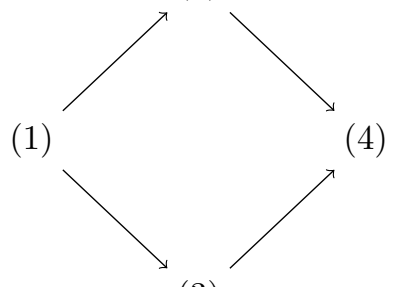

(3)

which we require to be commutative. For (2), we need to observe that $q$, as a refinement of $p$, induces a partition of each part of $p$; i.e. if $p$ has parts $p_{i}$ (of size $n_{i}$, in the notation of the definition), then $q$ gives a partition of each set $p_{i}$ into subsets $q_{i j}$ of size $n_{i j}$, giving an overall partition of $\{1, \ldots, n\}$ into parts of size $n_{i^{\prime}}$. Then we have two equivalences

$$
\left.\left.\left.\left.\left.\mathscr{G}_{n}\right|_{X_{p}^{n}}\right|_{X_{q}^{n}} \cong\left(\bigotimes_{i} \mathscr{G}_{n_{i}}\right)\right|_{X_{p}^{n}}\right|_{X_{q}^{n}} \cong\left(\bigotimes_{i} \bigotimes_{j} \mathscr{G}_{n_{i j}}\right)\right|_{X_{q}^{n}},\left.\left.\quad \mathscr{G}_{n}\right|_{X_{q}^{n}} \cong\left(\bigotimes_{i^{\prime}} \mathscr{G}_{n_{i^{\prime}}}\right)\right|_{X_{q}^{n}}
$$

where both pairs of termina are naturally identified; the factorization data then requires a 2-isomorphism between these 1-morphisms, and that these 2-isomorphisms are compatible in the same way as above. Finally, (3) is actually a special case of (2) where the parts of $p$ are reordered singletons, and the fact that this is an $S_{n}$-action is encoded in the above morphisms and compatibilities.

The most important special case of this definition is when $n=2$; then, we have the following translations of the three points:

(1) $\left.\mathscr{G}_{2}\right|_{\Delta} \cong \mathscr{G}_{1}$.

(2) $\left.\left.\mathscr{G}_{2}\right|_{X^{2} \backslash \Delta} \cong\left(\mathscr{G}_{1} \otimes \mathscr{G}_{1}\right)\right|_{X^{2} \backslash \Delta}$.

(3) There is an $S_{2}$-equivariance structure (as in Definition I.5.6) on $\mathscr{G}_{2}$ whose restriction to $\Delta$ is trivialized and whose restriction to $X^{2} \backslash \Delta$, via (2), is made isomorphic to the natural commutativity of the tensor product.

It should be noted that since $S_{n}$ is generated by transpositions, the compatibility of the general case of point Definition II.2.1] (3) with (2) can be reduced to point (3) above.

\section{II.3. Classification: the tOrus CASE}

Let $\mathscr{T}_{n}$ be an sf gerbe for $T$. Using Proposition II.1.2, we see that $\mathrm{Gr}_{2}$ breaks up into irreducible components $\mathrm{Gr}_{2}^{\lambda, \mu}$ isomorphic to $X^{2}$. Then on each, the above part (3) of the factorizable structure is largely redundant: by Lemma 1.5.1] [3), the $S_{2}$-equivariance is that unique equivariance structure extending the natural one on $\mathscr{G}_{1} \otimes \mathscr{G}_{1}$ over $X^{2} \backslash \Delta$, using the equivalence of (2). Thus, the only provision remaining is that this structure be trivialized on $\Delta$. 
For each $\lambda \in \Lambda_{T}$, on $\mathrm{Gr}_{2}^{\lambda, \lambda}=X^{2}$ we have a factorization isomorphism

$$
f^{\lambda, \lambda}:\left.\left.\left(\mathscr{T}_{1}^{\lambda} \otimes \mathscr{T}_{1}^{\lambda}\right)\right|_{X^{2} \backslash \Delta} \rightarrow \mathscr{T}_{2}^{\lambda, \lambda}\right|_{X^{2} \backslash \Delta}
$$

as above. This is in fact a meromorphic map of gerbes on $X^{2}$ having some order $a^{\lambda, \lambda}$; by Lemma I.5.7 and the above comments on point (3), we have a uniquely specified square root $b^{\lambda}$ of $a^{\lambda, \lambda}$. More generally, on the component $\operatorname{Gr}_{2}^{\lambda, \mu} \cong X^{2}$, the factorization equivalence

$$
f^{\lambda, \mu}:\left.\left.\left(\mathscr{T}_{1}^{\lambda} \otimes \mathscr{T}_{1}^{\mu}\right)\right|_{X^{2} \backslash \Delta} \rightarrow \mathscr{T}_{2}^{\lambda, \mu}\right|_{X^{2} \backslash \Delta}
$$

has some order $a^{\lambda, \mu}$.

Definition II.3.1. The quadratic form $Q=Q\left(\left\{\mathscr{T}_{n}\right\}\right)$ associated with the sf gerbe $\mathscr{T}_{n}$ is the map $Q: \Lambda_{T} \rightarrow A$ with $Q(\lambda)=b^{\lambda}$ above. The bilinear form $\kappa=\kappa\left(\left\{\mathscr{T}_{n}\right\}\right)$ associated with $\mathscr{T}_{n}$ is the map $\kappa: \Lambda_{T} \times \Lambda_{T} \rightarrow A$ with $\kappa(\lambda, \mu)=a^{\lambda, \mu}$.

The notation $\kappa$ is chosen because this form is closely related to the Killing form on $\Lambda$ when $G$ is taken to be a more general reductive group. The terminology "quadratic form" and "bilinear form" is chosen because we will presently prove that these functions are exactly that. Before beginning the proof, we observe that

$$
\left.\mathcal{O}_{X^{2}}(\Delta)^{\log a}\right|_{\Delta}=\mathcal{T}_{X}^{\log a}
$$

Indeed, $\Delta^{*} \mathcal{O}_{X^{2}}(\Delta) \cong \mathcal{T}_{X}$, where $\mathcal{T}_{X}$ is the tangent bundle on $X$.

Lemma II.3.2. We have $\mathscr{T}_{1}^{\lambda+\mu} \cong \mathscr{T}_{1}^{\lambda} \otimes \mathscr{T}_{1}^{\mu} \otimes \mathcal{T}_{X}^{\log \kappa(\lambda, \mu)}$.

Proof. We restrict to $\Delta$ the defining equivalence $\mathscr{T}_{2}^{\lambda, \mu} \cong \mathscr{T}_{1}^{\lambda} \otimes \mathscr{T}_{1}^{\mu} \otimes \mathcal{O}(\Delta)^{\log \kappa(\lambda, \mu)}$.

Proposition II.3.3. $\kappa$ is a symmetric bilinear form on $\Lambda_{T}$.

Proof. We will show that $\kappa(\lambda+\mu, \nu)=\kappa(\lambda, \nu) \kappa(\mu, \nu)$ and that $\kappa$ is symmetric. The latter is easy: the action of $S_{2}$ on $\mathrm{Gr}_{2}$ switches the irreducible components $\mathrm{Gr}_{2}^{\lambda, \mu}$ and $\mathrm{Gr}_{2}^{\mu, \lambda}$ and the $S_{2}$-equivariance of the factorization equivalence (2) ensures that the order is the same on both.

For the first, we consider the factorization equivalence on $\mathrm{Gr}_{3}^{\lambda, \mu, \nu}$,

$$
\left.\left.\left(\mathscr{T}_{1}^{\lambda} \otimes \mathscr{T}_{1}^{\mu} \otimes \mathscr{T}_{1}^{\nu}\right)\right|_{X_{p}^{3}} \rightarrow \mathscr{T}_{3}^{\lambda, \mu, \nu}\right|_{X_{p}^{3}},
$$

where $p$ is the partition $\{1,2,3\}=\{1\} \cup\{2\} \cup\{3\}$, and compute its order about the divisors $\Delta_{2,3}, \Delta_{1,3}$, and $\Delta_{1,2}$. For $\Delta_{2,3}$, now let $q$ be the partition $\{1,2,3\}=$ $\{1\} \cup\{2,3\}$ and consider the decomposition of Proposition II.1.3) (2), restricted to $\mathrm{Gr}_{3}^{\lambda, \mu, \nu}$ :

$$
\left.\left(\mathrm{Gr}_{1}^{\lambda} \times \mathrm{Gr}_{2}^{\mu, \nu}\right)\right|_{X_{q}^{3}}=\left.\mathrm{Gr}_{3}^{\lambda, \mu, \nu}\right|_{X_{q}^{3}}
$$

This decomposition takes place over $X \times X^{2}$ and in particular the diagonal $\Delta_{2,3}$ lies below the second factor. We get the more specific factorization equivalence

$$
\left.\left.\left(\mathscr{T}_{1}^{\lambda} \otimes \mathscr{T}_{2}^{\mu, \nu}\right)\right|_{X_{q}^{3}} \rightarrow \mathscr{T}_{3}^{\lambda, \mu, \nu}\right|_{X_{q}^{3}}
$$

which, therefore, has order $a^{\mu, \nu}=\kappa(\mu, \nu)$ about $\Delta_{2,3}$; by the compatibilities of Definition II.2.1 (2), this is the same as the order of (II.3) about $\Delta_{2,3}$. The same argument works for $\Delta_{1,3}$, giving order $\kappa(\lambda, \nu)$, and for $\Delta_{1,2}$, giving order $\kappa(\lambda, \mu)$. We find that

$$
\mathscr{T}_{3}^{\lambda, \mu, \nu} \cong \mathscr{T}_{1}^{\lambda} \otimes \mathscr{T}_{1}^{\mu} \otimes \mathscr{T}_{1}^{\nu} \otimes \mathcal{O}\left(\Delta_{13}\right)^{\log \kappa(\lambda, \nu)} \otimes \mathcal{O}\left(\Delta_{23}\right)^{\log \kappa(\mu, \nu)} \otimes \mathcal{O}\left(\Delta_{12}\right)^{\log \kappa(\lambda, \mu)} .
$$


We restrict to $\Delta_{12} \cong X^{2}$ and use equation (II.2) and Lemma II.3.2.

$$
\begin{aligned}
\mathscr{T}_{2}^{\lambda+\mu, \nu} & \cong\left(\mathscr{T}_{1}^{\lambda} \otimes \mathscr{T}_{1}^{\mu}\right) \otimes \mathscr{T}_{1}^{\nu} \otimes \mathcal{O}(\Delta)^{\log \kappa(\lambda, \nu) \kappa(\mu, \nu)} \otimes \operatorname{pr}_{1}^{*} \mathcal{T}_{X}^{\kappa(\lambda, \mu)} \\
& \cong \mathscr{T}_{1}^{\lambda+\mu} \otimes \mathscr{T}_{1}^{\nu} \otimes \mathcal{O}(\Delta)^{\log \kappa(\lambda, \nu) \kappa(\mu, \nu)} .
\end{aligned}
$$

This equivalence extends the factorization equivalence defined on $X^{2} \backslash \Delta$ and the notation includes an equality of orders, so we have $\kappa(\lambda+\mu, \nu)=\kappa(\lambda, \nu) \kappa(\mu, \nu)$.

Proposition II.3.4. $Q$ is a quadratic form on $\Lambda_{T}$. More precisely, its associated bilinear form is $\kappa$, in that we have

$$
Q(\lambda+\mu)=Q(\lambda) Q(\mu) \kappa(\lambda, \mu) .
$$

Proof. We analyze the factorization equivalence

$$
\left.\left.\left(\mathscr{T}_{1}^{\lambda} \otimes \mathscr{T}_{1}^{\mu} \otimes \mathscr{T}_{1}^{\lambda} \otimes \mathscr{T}_{1}^{\mu}\right)\right|_{X_{p}^{4}} \rightarrow \mathscr{T}_{4}^{\lambda, \mu, \lambda, \mu}\right|_{X_{p}^{4}}
$$

where $p$ is the partition $\{1,2,3,4\}=\{1\} \cup\{2\} \cup\{3\} \cup\{4\}$. There are six exceptional divisors where any two of the four coordinates come together, and we compute the order of this equivalence about each one, then restrict appropriately as in the proof of Proposition II.3.3.

The cases of the divisors $\Delta_{1,2}, \Delta_{1,4}, \Delta_{2,3}$, and $\Delta_{3,4}$ are essentially the same, so we do the first one and state the results for the others. For $\Delta_{1,2}$ we form the partition $q:\{1,2,3,4\}=\{1,2\} \cup\{3\} \cup\{4\}$ and consider the more specific factorization equivalence (through which (II.4) factors),

$$
\left.\left.\left(\mathscr{T}_{2}^{\lambda, \mu} \otimes \mathscr{T}_{1}^{\lambda} \otimes \mathscr{T}_{2}^{\mu}\right)\right|_{X_{q}^{4}} \rightarrow \mathscr{T}_{4}^{\lambda, \mu, \lambda, \mu}\right|_{X_{q}^{4}}
$$

which evidently shows that (II.4) has order $\kappa(\lambda, \mu)$ about $\Delta_{1,2}$. The same analysis shows that in fact we get this order for all four of the named divisors.

The two remaining divisors $\Delta_{1,3}$ and $\Delta_{2,4}$ give, respectively, orders of $\kappa(\lambda, \lambda)$ and $\kappa(\mu, \mu)$ by the same reasoning, but we prefer to write them as $Q(\lambda)^{2}$ and $Q(\mu)^{2}$ in preparation for what is to come. Thus, (II.4) becomes

$$
\begin{aligned}
\mathscr{T}_{4}^{\lambda, \mu, \lambda, \mu} & \cong \mathscr{T}_{1}^{\lambda} \otimes \mathscr{T}_{1}^{\mu} \otimes \mathscr{T}_{1}^{\lambda} \otimes \mathscr{T}_{1}^{\mu} \otimes \mathcal{O}\left(\Delta_{1,2}\right)^{\log \kappa(\lambda, \mu)} \otimes \mathcal{O}\left(\Delta_{3,4}\right)^{\log \kappa(\lambda, \mu)} \\
& \otimes \mathcal{O}\left(\Delta_{2,3} \cup \Delta_{1,4}\right)^{\log \kappa(\lambda, \mu)} \otimes \mathcal{O}\left(\Delta_{1,3}\right)^{\log Q(\lambda)^{2}} \otimes \mathcal{O}\left(\Delta_{2,4}\right)^{\log Q(\mu)^{2}} .
\end{aligned}
$$

We restrict to the diagonal $\Delta_{1,2} \cap \Delta_{3,4} \cong \mathrm{Gr}_{2}^{\lambda+\mu, \lambda+\mu} \cong X^{2}$, by Proposition II.1.2. Then all but $\Delta_{1,2}$ and $\Delta_{3,4}$ become the main diagonal $\Delta$, while we have

$$
\left.\mathcal{O}\left(\Delta_{1,2}\right)^{\log \kappa(\lambda, \mu)}\right|_{\Delta_{1,2} \cap \Delta_{3,4}}=\left.\left.\mathcal{O}\left(\Delta_{1,2}\right)^{\log \kappa(\lambda, \mu)}\right|_{\Delta_{1,2}}\right|_{\Delta_{3,4}}=\operatorname{pr}_{1}^{*} \mathcal{T}_{X}^{\kappa(\lambda, \mu)}
$$

and likewise we have $\left.\mathcal{O}\left(\Delta_{3,4}\right)^{\log \kappa(\lambda, \mu)}\right|_{\Delta_{1,2} \cap \Delta_{3,4}} \cong \operatorname{pr}_{2}^{*} \mathcal{T}_{X}^{\kappa(\lambda, \mu)}$. Therefore, after restricting and applying Lemma II.3.2, equation (II.5) becomes

$$
\mathscr{T}_{2}^{\lambda+\mu, \lambda+\mu} \cong \mathscr{T}_{1}^{\lambda+\mu} \otimes \mathscr{T}_{1}^{\lambda+\mu} \otimes \mathcal{O}(\Delta)^{\log Q(\lambda)^{2} Q(\mu)^{2} \kappa(\lambda, \mu)^{2}}
$$

As in Proposition II.3.3 this is an equality of orders, and so by definition we have

$$
Q(\lambda+\mu)^{2}=Q(\lambda)^{2} Q(\mu)^{2} \kappa(\lambda, \mu)^{2} .
$$

In order to take the square root, we simply apply Lemma I.5.7 to the coordinateswapping action of $S_{2}$ along with provision Definition II.2.1] (3), as follows: consider the larger action of $S_{2}$ on $X^{4}$ which swaps the first and last pairs of coordinates; it exchanges $\Delta_{1,4}$ with $\Delta_{2,3}$ and fixes both $\Delta_{1,3}$ and $\Delta_{2,4}$, which are the four divisors whose corresponding orders appear in equation (II.6) Thus, the square root of 
$\kappa(\lambda, \mu)^{2}$ extracted is indeed $\kappa(\lambda, \mu)$ (the order about either one of the first pair) and those of $Q(\lambda)^{2}$ and $Q(\mu)^{2}$ are, respectively, $Q(\lambda)$ and $Q(\mu)$, since that is how $Q$ is defined. This completes the proof.

Now that we have proved that $Q$ is a quadratic form, we can come full circle and show that it effectively determines the entire factorizable structure of the $\mathscr{T}_{n}$. To state this, we make the definition:

Definition II.3.5. Let $\mathbf{H}_{\mathrm{sf}}^{2}(T, X, A)$ be the 2-Picard category whose objects are sf $A$-gerbes on $\operatorname{Gr}_{T, X^{n}}$. Let $Q\left(\Lambda_{T}, A\right)$ be the group of $A$-valued quadratic forms on $\Lambda_{T}$.

In this language, Proposition II.3.4 together with the defintion of $Q$ shows that we have a homomorphism $\mathbf{H}_{\mathrm{sf}}^{2}(T, X, A) \rightarrow Q\left(\Lambda_{T}, A\right)$. This extends to the following theorem:

Proposition II.3.6. The above homomorphism fits into a split short exact sequence of 2-Picard categories,

$$
1 \rightarrow \operatorname{Hom}\left(\Lambda_{T}, \mathbf{H}^{2}(X, A)\right) \rightarrow \mathbf{H}_{s f}^{2}(T, X, A) \rightarrow Q\left(\Lambda_{T}, A\right) \rightarrow 1 .
$$

Here, the first term $\operatorname{Hom}\left(\Lambda_{T}, \mathbf{H}^{2}(X, A)\right)$ refers to the category of homomorphisms from $\Lambda$ into the 2-Picard category $\mathbf{H}^{2}(X, A)$ or, in other words, of "commutative multiplicative $A$-gerbes", as given in Definition I.3.3. The concept of an exact sequence of 2-Picard categories was described before Proposition I.1.7.

Proof. The easiest part of the proposition is that the automorphism category of the trivial sf gerbe is identified with that of commutative multiplicative $A$-torsors for $\Lambda_{T}$. Indeed, it is (by definition) the category of "sf $A$-torsors" on $\mathrm{Gr}_{T}$, but since all the diagonals in $X^{n}$ are Zariski-closed, the factorization isomorphisms extend across them and become a multiplicative structure, commutative by virtue of $S_{n}$-equivariance. Likewise for their 2-morphisms (i.e. sf maps to $A$ ).

Suppose we are given such a multiplicative gerbe $\left\{\mathscr{T}^{\lambda}\right\}$ and a quadratic form $Q$; we will construct an sf gerbe $\left\{\mathscr{T}_{n}\right\}$ in a manner compatible with multiplication. We set $\kappa$ to be the bilinear form defined by $Q$ as in Proposition II.3.4. First, let

$$
\mathscr{T}_{1}^{\lambda}=\mathscr{T}^{\lambda} \otimes \mathcal{T}_{X}^{\log Q(\lambda)} .
$$

This defines $\mathscr{T}_{1}$ by defining it on each connected component of $\operatorname{Gr}_{T, X}$. In general, to define $\mathscr{T}_{n}$ we need only define its restriction to each component copy $\mathrm{Gr}_{T, X^{n}}^{\lambda_{1}, \ldots, \lambda_{n}} \cong X^{n}$ and give isomorphisms of these restrictions on the diagonals described in Proposition II.1.2. Here, we take

$$
\mathscr{T}_{n}^{\lambda_{1}, \ldots, \lambda_{n}}=\mathscr{T}_{1}^{\lambda_{1}} \otimes \cdots \otimes \mathscr{T}_{1}^{\lambda_{n}} \otimes \bigotimes_{i, j} \mathcal{O}\left(\Delta_{i, j}\right)^{\log \kappa\left(\lambda_{i}, \lambda_{j}\right)},
$$

where as in the previous proofs, $\Delta_{i, j}$ is the divisor where $x_{i}=x_{j}$. We show how to define the factorization equivalences for $n=2$ : there, we take

$$
\begin{aligned}
\left.\mathscr{T}_{2}^{\lambda, \mu}\right|_{\Delta} & =\left.\mathscr{T}_{1}^{\lambda} \otimes \mathscr{T}_{1}^{\mu} \otimes \mathcal{O}(\Delta)^{\log \kappa(\lambda, \mu)}\right|_{\Delta} \\
& =\mathscr{T}^{\lambda} \otimes \mathscr{T}^{\mu} \otimes \mathcal{T}_{X}^{\log Q(\lambda) Q(\mu) \kappa(\lambda, \mu)} \cong \mathscr{T}^{\lambda+\mu} \otimes \mathcal{T}_{X}^{\log Q(\lambda+\mu)}=\mathscr{T}_{1}^{\lambda+\mu},
\end{aligned}
$$

and let the equivalences

$$
\left.\left.\left(\mathscr{T}_{1}^{\lambda} \otimes \mathscr{T}_{1}^{\mu}\right)\right|_{X^{2} \backslash \Delta} \cong \mathscr{T}_{2}^{\lambda+\mu}\right|_{X^{2} \backslash \Delta}
$$


be the natural ones induced by the trivialization of $\mathcal{O}(\Delta)^{\log \kappa(\lambda, \mu)}$. As noted after Definition II.2.1 this suffices to define the $S_{2}$-equivariance of $\mathscr{T}_{2}$ as well: it is the unique equivalence $\phi: \mathscr{T}_{2} \rightarrow s^{*} \mathscr{T}_{2}$ (where $s$ is the involution defined by swapping coordinates in $X^{2}$ ) extending the natural one on tensor products using the above factorization on $X^{2} \backslash \Delta$; this is the same as taking the product of the natural symmetry of the tensor product with the $S_{2}$-equivariance of $\mathcal{O}(\Delta)^{\log \kappa(\lambda, \mu)}$ induced by the invariance of the trivial gerbe on $X^{2} \backslash \Delta$. We define its trivialization on $\Delta$ to be that arising (in reference to the equations (II.9) from the equivalence of $S_{2}$-equivariant gerbes on $X^{2}$ :

$$
\mathcal{O}(\Delta)^{\log \kappa(\lambda, \mu)} \otimes \mathcal{O}(\Delta)^{\log Q(\lambda)} \otimes \mathcal{O}(\Delta)^{\log Q(\mu)} \cong \mathcal{O}(\Delta)^{\log Q(\lambda+\mu)} .
$$

With these definitions, it is clear that $\mathscr{T}_{2}$ defines the bilinear form $\kappa$ and quadratic form $Q$. As an example of the higher factorizations, we verify the diagonal restrictions for $\mathscr{T}_{3}$ :

$$
\left.\mathscr{T}_{3}^{\lambda, \mu, \nu}\right|_{\Delta_{1,2}}=\left.\left(\mathscr{T}_{1}^{\lambda} \otimes \mathscr{T}_{1}^{\mu}\right) \otimes \mathscr{T}_{1}^{\nu} \otimes \mathcal{O}\left(\Delta_{1,2}\right)^{\log \kappa(\lambda, \mu)}\right|_{\Delta_{1,2}} \otimes \mathcal{O}(\Delta)^{\log \kappa(\lambda, \nu) \kappa(\mu, \nu)} .
$$

Since $\left.\mathcal{O}\left(\Delta_{1,2}\right)^{\log \kappa(\lambda, \mu)}\right|_{\Delta_{1,2}}=\operatorname{pr}_{1}^{*} \mathcal{T}_{X}^{\log \kappa(\lambda, \mu)}$, the first factor becomes $\mathscr{T}_{1}^{\lambda+\mu}$ as in (II.9), while for the remaining $\mathcal{O}(\Delta)$, we simplify the exponent using the bilinearity of $\kappa$. Similar considerations give the factorizations away from diagonals.

It is easy to see that the construction $\left(\left\{\mathscr{T}^{\lambda}\right\}, Q\right) \mapsto\left\{\mathscr{T}_{n}\right\}$ is a homomorphism

$$
\operatorname{Hom}\left(\Lambda_{T}, \mathbf{H}^{2}(X, A)\right) \times Q\left(\Lambda_{T}, A\right) \rightarrow \mathbf{H}_{\mathrm{sf}}^{2}(T, X, A) .
$$

The resulting sequence

$$
1 \rightarrow \operatorname{Hom}\left(\Lambda_{T}, \mathbf{H}^{2}(X, A)\right) \rightarrow \mathbf{H}_{\mathrm{sf}}^{2}(T, X, A) \rightarrow Q\left(\Lambda_{T}, A\right) \rightarrow 1
$$

is evidently exact at both the left and (by this construction) the right. If we have an sf gerbe $\mathscr{T}_{n}$ with $Q\left(\mathscr{T}_{n}\right)=1$ the trivial form, then Lemma II.3.2 shows that the $\mathscr{T}_{1}^{\lambda}$ are themselves a commutative multiplicative gerbe on $X \times \Lambda$, and so $\mathscr{T}_{n}$ is in fact defined by this multiplicative gerbe by the above construction. This gives exactness in the middle and completes the proof.

\section{II.4. Multiplicative FaCtorizable Gerbes}

It is evident that the concepts of factorizability and multiplicativity are closely related. In this section we combine the two and discuss their interactions.

Definition II.4.1. An sf $A$-gerbe $\mathscr{T}_{n}$ on $\mathrm{Gr}_{T, X^{n}}$ forms a multiplicative sf gerbe if the factorization equivalences of Definition II.2.1](2) extend across the exceptional diagonals using the multiplicative structure (II.1) of $\mathrm{Gr}_{T, X^{n}}$.

Note that, by Lemma I.5.1 if this extension exists it is unique, so this is indeed a condition rather than a structure. Also note that, denoting by $m_{n}$ the multiplication on $\mathrm{Gr}_{T, X^{n}}$, the induced equivalences $\mathscr{T}_{n} \otimes \mathscr{T}_{n} \cong m_{n}^{*} \mathscr{T}_{n}$ are the structure of a commutative multiplicative $A$-gerbe (in particular, $\mathscr{T}_{1}$ is such a gerbe as made explicit above). This definition is inspired by the following phenomenon for sections of $A$ :

Lemma II.4.2. Let $f: \Lambda_{T} \rightarrow A$ be a function. Then the following are equivalent:

(1) $f$ is a homomorphism.

(2) There exists a unique factorizable collection $f_{n}: \mathrm{Gr}_{T, X^{n}} \rightarrow A$ of locally constant functions such that $f_{1} \mid \mathrm{Gr}_{T, X}^{\lambda}=f(\lambda)$. 
(3) The above $f_{n}$ are actually multiplicative factorizable.

Proof. (1) $\Longrightarrow(2)$. We set $f_{n} \mid \mathrm{Gr}_{T, X^{n}}^{\lambda_{1}, \ldots, \lambda_{n}}=f\left(\lambda_{1}+\cdots+\lambda_{n}\right)=f\left(\lambda_{1}\right) \cdots f\left(\lambda_{n}\right)$; then, given the intersection pattern of Proposition II.1.2 the fact that $f$ is a homomorphism makes this a factorizable function.

$(2) \Longrightarrow(3)$. Since the $f_{n}$ are locally constant, the factorization equalities extend across the exceptional diagonals.

$(3) \Longrightarrow(1)$. The equation $f(\lambda+\mu)=f(\lambda) f(\mu)$ follows from the multiplicativity property applied to the map $\mathrm{Gr}_{T, X} \times \mathrm{Gr}_{T, X} \rightarrow \mathrm{Gr}_{T, X}^{2}$, when restricted to the diagonal in $X^{2}$ (which in the latter is again $\operatorname{Gr}_{T, X}$ ).

Unlike for functions, the factorization equivalences do not automatically extend to a multiplicative sf structure for gerbes. By definition, part of this failure is measured by the associated bilinear form $\kappa$, and in fact, this is the entire obstruction:

Proposition II.4.3. Suppose that $\mathscr{T}_{n}$ is an sf A-gerbe on $\mathrm{Gr}_{T, X^{n}}$ whose associated bilinear form is trivial. Then its factorization equivalences extend to the structure of a multiplicative sf gerbe. Furthermore, if $\mathscr{T}_{n}$ is the trivial gerbe, then the associativity constraint is identified with $1 \in A$ and the commutativity constraint of $\mathscr{T}_{1}^{\lambda} \otimes \mathscr{T}_{1}^{\mu}$ with $Q(\lambda) Q(\mu)$.

We note that by factorizability, the commutativity of a more general product $\mathscr{T}_{n}^{\lambda_{1}, \ldots, \lambda_{n}} \otimes \mathscr{T}_{n}^{\mu_{1}, \ldots, \mu_{n}}$ is therefore $Q\left(\lambda_{1}\right) \ldots Q\left(\mu_{n}\right)$, so it is only necessary to compute the one in the proposition.

Proof. That $\mathscr{T}_{1}$ is multiplicative comes from Lemma II.3.2, Consider the general factorization equivalence $\mathscr{T}_{n} \otimes \mathscr{T}_{m} \cong m_{n, m}^{*} \mathscr{T}_{n+m}$ defined away from the exceptional diagonals in the map $m_{n, m}$ of (II.1). On the component $\mathrm{Gr}_{n}^{\lambda_{1}, \ldots, \lambda_{n}} \times \mathrm{Gr}_{m}^{\mu_{1}, \ldots, \mu_{m}} \cong$ $X^{n} \times X^{m}$, let $x_{1}, \ldots, x_{n}$ and $y_{1}, \ldots, y_{m}$ be the coordinates and $\Delta_{i j}=\left\{x_{i}=y_{j}\right\}$ one of the exceptional diagonals; the order of the above equivalence about $\Delta_{i j}$ is $\kappa\left(\lambda_{i}, \mu_{j}\right)$ by (II.8) This shows that the $\mathscr{T}_{n}$ are in fact multiplicative factorizable when $\kappa$ is trivial.

To compute the associativity constraint of each $\mathscr{T}_{n}$, as in the comment above we need only compute it for $\mathscr{T}_{1}$, for which we consider, as in Proposition II.3.3, the component $\mathrm{Gr}_{3}^{\lambda, \mu, \nu} \subset \mathrm{Gr}_{3}$. By restricting $\mathscr{T}_{3}^{\lambda, \mu, \nu}$ successively along the factorization maps

$$
\mathrm{Gr}_{1}^{\lambda} \times \mathrm{Gr}_{1}^{\mu} \times \mathrm{Gr}_{1}^{\nu} \rightarrow \mathrm{Gr}_{2}^{\lambda, \mu} \times \mathrm{Gr}_{1}^{\nu} \rightarrow \mathrm{Gr}_{3}^{\lambda, \mu, \nu}
$$

and applying factorizability of $\mathscr{T}_{3}$, we obtain the isomorphism

$$
\left(\mathscr{T}_{1}^{\lambda} \otimes \mathscr{T}_{1}^{\mu}\right) \otimes \mathscr{T}_{1}^{\nu} \cong \mathscr{T}_{1}^{\lambda, \mu, \nu} .
$$

Likewise, using $\mathrm{Gr}_{2}^{\mu, \nu}$ in the second stage we get the other bracketing. But either way the maps are obtained by restriction of the factorization of $\mathscr{T}_{3}^{\lambda, \mu, \nu}$ to the smallest diagonal $\Delta_{123} \subset X^{3}$, and both these restrictions are equal.

To compute commutativity, we must show that the two equivalences

$$
\mathscr{T}_{1}^{\lambda} \otimes \mathscr{T}_{1}^{\mu} \cong \mathscr{T}_{1}^{\lambda+\mu}, \quad \mathscr{T}_{1}^{\mu} \otimes \mathscr{T}_{1}^{\lambda} \cong \mathscr{T}_{1}^{\lambda+\mu},
$$

differ by the constant multiple $Q(\lambda+\mu)=Q(\lambda) Q(\mu)$ (since $\kappa$ is trivial). Each of these is induced by the factorization equivalences

$$
\mathscr{T}_{1}^{\lambda} \otimes \mathscr{T}_{1}^{\mu} \cong \mathscr{T}_{2}^{\lambda, \mu}, \quad \mathscr{T}_{1}^{\mu} \otimes \mathscr{T}_{1}^{\lambda} \cong \mathscr{T}_{2}^{\mu, \lambda},
$$


upon restriction to the diagonal in $X^{2}$, and these are obtained from each other by swapping coordinates. We consider, as in Proposition II.3.4 the component $\mathrm{Gr}_{4}^{\lambda, \mu, \lambda, \mu} \subset \mathrm{Gr}_{4}$, and write the pair of equivalences, obtained from factorization,

$$
\mathscr{T}_{1}^{\lambda} \otimes \mathscr{T}_{1}^{\mu} \otimes \mathscr{T}_{1}^{\mu} \otimes \mathscr{T}_{1}^{\lambda} \cong \mathscr{T}_{4}^{\lambda, \mu, \mu, \lambda} \cong \mathscr{T}_{2}^{\lambda, \mu} \otimes \mathscr{T}_{2}^{\mu, \lambda}
$$

where, if we bracket the first and second pairs on the left, the composed equivalence is the product of the two exhibited above. We restrict the second half of this equation to the diagonal $\Delta_{12,34}=\left\{x_{1}=x_{2}, x_{3}=x_{4}\right\}$, obtaining

$$
\mathscr{T}_{2}^{\lambda+\mu, \lambda+\mu} \cong \mathscr{T}_{1}^{\lambda+\mu} \otimes \mathscr{T}_{1}^{\mu+\lambda},
$$

where of course $\lambda+\mu=\mu+\lambda$. If $\mathscr{T}_{1}$ is trivialized, we may use the first half of the previous equation to trivialize both sides and, comparing with the proof of Lemma I.5.7, we see that indeed the coordinate swap makes these trivializations differ by $Q(\lambda+\mu)$. Since $\kappa$ is trivial, $Q$ is a homomorphism, so this is the same as $Q(\lambda) Q(\mu)$.

We give an alternative description of such gerbes, beginning by constructing a certain sf sheaf of groups.

Definition II.4.4. For any sheaf of abelian groups $\mathcal{A}$ on $X$, let $\operatorname{Fact}(\mathcal{A})_{n}$ be the following sheaf on $X^{n}$ : for every $U \subset X^{n}, \operatorname{Fact}(\mathcal{A})_{n}(U)$ is the subsheaf of $\mathcal{A}^{n}$ subject only to the restrictions that if $U \cap \Delta_{i j} \neq \varnothing$, then $\operatorname{Fact}(\mathcal{A})(U) \subset \Delta_{i j}$ (the first diagonal in $X^{n}$, the second in $\left.\mathcal{A}^{n}\right)$. If $\mathcal{A}=\underline{A}$ is the constant sheaf on a discrete abelian group $A$, we will just write $\operatorname{Fact}(A)_{n}$.

It should be noted that $\operatorname{Fact}(\mathcal{A})_{n}$ is not factorizable in the same way that $\operatorname{Gr}_{T, X^{n}}$ is, as described in Proposition II.1.2, since it degenerates to subsets over the diagonals rather than quotients. In fact, the two are related by the following constructions:

Lemma II.4.5. Let $T$ be an algebraic torus, ${ }^{L} T$ the dual torus with

$$
\operatorname{Hom}\left(\mathbf{G}_{\mathbf{m}},{ }^{L} T\right)=\operatorname{Hom}\left(T, \mathbf{G}_{\mathbf{m}}\right), \quad \operatorname{Hom}\left({ }^{L} T, \mathbf{G}_{\mathbf{m}}\right)=\operatorname{Hom}\left(\mathbf{G}_{\mathbf{m}}, T\right) ;
$$

then we have

$$
\mathcal{H} \mathrm{om}\left(\operatorname{Fact}\left({ }^{L} T\right)_{n}, \mathbf{G}_{\mathbf{m}} \times X^{n}\right) \cong \mathrm{Gr}_{T, X^{n}}
$$

as sheaves of groups over $X^{n}$ (note that ${ }^{L} T$ refers to the sheaf of groups represented by the group scheme ${ }^{L} T \times X$ over $X$, and not the constant sheaf on the abelian group ${ }^{L} T(\mathbb{C})$. This is the only time we use a non-discrete group in the $\operatorname{Fact}(\mathcal{A})_{n}$ construction).

Conversely, let $A$ be a (discrete) abelian group and denote

$$
{ }^{L} T(A)=\operatorname{Hom}\left(\Lambda_{T}, A\right) \cong \Lambda^{T} \otimes A .
$$

Then we can identify the pushforward to $X^{n}$ of the constant sheaf $\underline{A}$ on $\operatorname{Gr}_{T, X^{n}}$ with $\operatorname{Fact}\left({ }^{L} T(A)\right)_{n}$.

Proof. Let $U \subset X^{n}$ be the complement of all the diagonals $\Delta_{i j}$; then since we have $\left.\operatorname{Fact}\left({ }^{L} T\right)_{n}\right|_{U} \cong{ }^{L} T^{n}$ and $\operatorname{Hom}\left({ }^{L} T, \mathbf{G}_{\mathbf{m}}\right) \cong \Lambda^{L} T=\Lambda_{T}$, we have

$$
\left.\mathcal{H} \mathrm{om}\left(\operatorname{Fact}\left({ }^{L} T\right)_{n}, \mathbf{G}_{\mathbf{m}} \times X^{n}\right)\right|_{U} \cong\left(\Lambda^{L} T\right)^{n} \times X^{n}=\Lambda_{T}^{n} \times X^{n} .
$$

We use Proposition II.1.2 to construct an isomorphism with $\operatorname{Gr}_{T, X^{n}}$ over $U$ and use the same indexing convention for the components. The first part of the lemma would follow if we could show that the closure of $\mathcal{H}$ om $\left.\left(\operatorname{Fact}\left({ }^{L} T\right)_{n}, \mathbf{G}_{\mathbf{m}} \times X^{n}\right)\right|_{U} ^{\lambda_{1}, \ldots, \lambda_{n}}$ 
intersects, over $\Delta_{i j}$, all the $\left.\mathcal{H o m}\left(\operatorname{Fact}\left({ }^{L} T\right)_{n}, \mathbf{G}_{\mathbf{m}} \times X^{n}\right)\right|_{U} ^{\mu_{1}, \ldots, \mu_{n}}$ with $\lambda_{i}+\lambda_{j}=$ $\mu_{i}+\mu_{j}$ and all other $\lambda_{k}=\mu_{k}$. To see this, let $V$ be any neighborhood intersecting only $\Delta_{i j}$, so that the restriction map

$$
\left.\left.{ }^{L} T^{n-1} \cong \operatorname{Fact}\left({ }^{L} T\right)_{n}\right|_{V} \rightarrow \operatorname{Fact}\left({ }^{L} T\right)\right|_{V \backslash \Delta_{i j}} \cong{ }^{L} T^{n}
$$

is the inclusion of ${ }^{L} T^{n-1}$ as the $i j$ th diagonal of ${ }^{L} T^{n}$. The corresponding map $\Lambda_{T}^{n} \rightarrow \Lambda_{T}^{n-1}$ is the summation of the $i$ th and $j$ th coordinates, as desired.

For the second part, we use Proposition II.1.2 to identify the connected components of $\operatorname{Gr}_{T, X^{n}}$ over any open set $V \subset X^{n}$ : when $V \subset U$ they correspond to $\Lambda_{T}^{n}$, so that locally constant functions on $V$ are identified with $\operatorname{Hom}\left(\Lambda_{T}^{n}, A\right)={ }^{L} T(A)^{n}$, by definition of the latter. For each diagonal $\Delta_{i j}$ intersecting $V$, components with the same sum of their $i$ th and $j$ th indexes are incident, so that the corresponding functions must have equal $i$ th and $j$ th coordinates in ${ }^{L} T(A)$. That is, locally constant functions on such $V$ are identified with diagonals in ${ }^{L} T(A)^{n}$, since as above the sum map for $\Lambda_{T}$ corresponds to the diagonal map for ${ }^{L} T$, as desired.

As in the lemma, let $A$ be a (discrete) abelian group, considered as a constant sheaf on $X$. We will need the following notion of sf comultiplicativity for gerbes $\mathscr{Z}_{n}$ over $\operatorname{Fact}(A)_{n}$. Let $p$ be a partition of $[1, n]$ into $m$ parts of sizes $n_{i}$, and let $X_{p}^{n}$ and $\Delta_{p}^{n} \cong X^{m}$ be, as in Proposition II.1.3. the open and diagonal subschemes determined by $p$ in $X^{n}$; then we have two homomorphisms of sheaves of groups:

$$
\begin{gathered}
\psi_{p}: \operatorname{Fact}(A)_{n} \hookrightarrow \prod_{i=1}^{m} \operatorname{pr}_{X^{n_{i}}}^{*} \operatorname{Fact}(A)_{n_{i}}, \\
\phi_{p}:\left.\operatorname{Fact}(A)_{m} \stackrel{\sim}{\rightarrow} \operatorname{Fact}(A)_{n}\right|_{\Delta_{p}^{n}},
\end{gathered}
$$

where $\phi_{p}$ is in fact an isomorphism, and $\psi_{p}$ is a generalization of the defining inclusion $\operatorname{Fact}(A)_{n} \hookrightarrow \underline{A}_{X^{n}}^{n}$. These homomorphisms, as for the factorizability of $\mathrm{Gr}_{n}$, come with numerous compatibilities when refinements of $p$ are given. Now we may make a definition.

Definition II.4.6. Let the $\mathscr{Z}_{n}$ be $\operatorname{Fact}(A)_{n}$-gerbes on $X^{n}$ given for all $n$. The structure of sf comultiplicativity is the data of equivalences for all partitions $p$ (recall the notation ${ }^{2} \phi$ for the change-of-group operation on gerbes associated with a group homomorphism of the coefficients, given in (I.1) $)$ :

$$
{ }^{2} \psi_{p}\left(\mathscr{Z}_{n}\right) \cong \prod_{i=1}^{m} \operatorname{pr}_{X^{n_{i}}}^{*}\left(\mathscr{Z}_{n_{i}}\right),\left.\quad{ }^{2} \phi_{p}\left(\mathscr{Z}_{m}\right) \cong \mathscr{Z}_{n}\right|_{\Delta_{p}^{n}},
$$

together with compatibilities as in Definition II.2.1. Likewise, we define sf multiplicative torsors and sections of $\operatorname{Fact}(A)_{n}$.

The concept of sf comultiplicativity is also related to factorizability on the grassmannian:

Lemma II.4.7. The first identification of Lemma II.4.5 (taken over all $n$ ) connects the map (II.10) with the multiplication of (II.1) and connects (II.11) with the diagonal part of the factorization data in Proposition II.1.3. The second identification (over all $n$ ) connects multiplicative factorizable A-valued functions with sf comultiplicative $\operatorname{Fact}(T(A))_{n}$-valued functions. 
Proof. Whereas in the previous construction, we used the fact that the sum map on $\Lambda_{T}$ induced and was induced by the diagonal map on ${ }^{L} T$, here we use the fact that the diagonal map on $\Lambda_{T}$ induces and is induced by the product map on ${ }^{L} T$. The details are otherwise the same.

The main goal of this section is to state and prove the analogue of the second part of Lemma II.4.7 for gerbes rather than functions. As is typical of constructions on gerbes, we must pass through torsors and functions as well by way of accounting for higher morphisms. The statement of the correspondence is straightforward:

Definition II.4.8. Let $\mathscr{Z}_{n}$ be an sf comultiplicative Fact $\left({ }^{L} T(A)\right)_{n}$-gerbe on $X^{n}$. Then the corresponding multiplicative sf $A$-gerbe on $\mathrm{Gr}_{T, X^{n}}$ is defined to be trivial above any open set in $X^{n}$ on which $\mathscr{Z}_{n}$ is trivial, with gluing data given by the (recursively defined) map from sf comultiplicative $\operatorname{Fact}\left({ }^{L} T(A)\right)_{n}$-torsors to multiplicative sf $A$-torsors on $\mathrm{Gr}_{T, X^{n}}$, applied to the gluing data of $\mathscr{Z}_{n}$.

Conversely, let $\mathscr{T}_{n}$ be a multiplicative sf $A$-gerbe on $\mathrm{Gr}_{T, X^{n}}$. Since $\Lambda_{T}$ is finitely generated, there is an open cover of $X^{n}$ above which $\mathscr{T}_{n}$ is trivial; we define the corresponding sf comultiplicative $\operatorname{Fact}\left({ }^{L} T(A)\right)_{n}$-gerbe $\mathscr{Z}_{n}$ on $X^{n}$ to be trivial on this cover, with gluing data given by the (recursively defined) map from multiplicative sf $A$-torsors on $\mathrm{Gr}_{T, X^{n}}$ to sf comultiplicative $\operatorname{Fact}\left({ }^{L} T(A)\right)_{n}$-torsors on $X^{n}$.

Proposition II.4.9. The constructions given in Definition II.4.8 are inverse equivalences of 2-categories between multiplicative sf $A$-torsors on $\mathrm{Gr}_{T, X^{n}}$ and sf comultiplicative Fact $\left({ }^{L} T(A)\right)_{n}$-gerbes on $X^{n}$. Furthermore, the $\mathscr{T}_{n}$ corresponding to a $\mathscr{Z}_{n}$ can be described as follows:

For each section $X^{n} \cong \mathrm{Gr}_{T, X^{n}}^{\lambda_{1}, \ldots, \lambda_{n}} \hookrightarrow \mathrm{Gr}_{T, X^{n}}$ as one of the irreducible components, let $\bar{\lambda}$ : $\operatorname{Fact}\left({ }^{L} T\right)_{n} \rightarrow \mathbf{G}_{\mathbf{m}} \times U$ be the corresponding homomorphism from Lemma II.4.5; we use the same notation for the induced map $\operatorname{Fact}\left({ }^{L} T(A)\right) \rightarrow \underline{A}_{X^{n}}$. Then we have

$$
\mathscr{T}_{n} \cong{ }^{2} \bar{\lambda}\left(\mathscr{Z}_{n}\right)
$$

and the multiplicative factorizable structure is the one obtained from the sf comultiplicative structure of $\mathscr{Z}_{n}$ and the first part of Lemma II.4.7.

Proof. We begin by establishing that the second part of the construction is valid. To expand on it, the claim is as follows: let $\vec{x} \in X^{n}$ and suppose that it has $k$ distinct coordinates, so that $\left.\mathrm{Gr}_{T, X^{n}}\right|_{\vec{x}} \cong \Lambda_{T}^{k}$. We choose finitely many generators $l_{1}, \ldots, l_{m}$ for $\Lambda_{T}^{k}$ and select a neighborhood $V$ about $\vec{x}$, in which all points have at least $k$ distinct coordinates, on which all the $\mathscr{T}_{n}^{l_{i}}$ are trivial; here, the connected components of $\left.\mathrm{Gr}_{T, X^{n}}\right|_{V}$ are indexed by $\Lambda_{T}^{k}$ compatibly with multiplication and the $\mathscr{T}_{n}^{l_{i}}$ refer to the parts of $\mathscr{T}_{n}$ on these components. Using the multiplicativity of $\left.\mathscr{T}_{n}\right|_{V}$ over $V$, we obtain trivializations of $\mathscr{T}_{n}^{l}$ for any $l \in \Lambda_{T}^{k}$.

For this to unambiguously define a trivialization of $\left.\mathscr{T}_{n}\right|_{V}$, it is necessary and sufficient that for any $l$ and any representation of $l$ as a linear combination of the $l_{i}$, the multiplications of the $\mathscr{T}_{n}^{l_{i}}$ are all isomorphic. For example, the equality $l_{i}+l_{j}=l=l_{j}+l_{i}$ corresponds to the two multiplications,

$$
\mathscr{T}_{n}^{l_{i}} \otimes \mathscr{T}_{n}^{l_{j}} \cong \mathscr{T}_{n}^{l_{1}+l_{j}}=\mathscr{T}_{n}^{l}=\mathscr{T}_{n}^{l_{j}+l_{i}} \cong \mathscr{T}_{n}^{l_{j}} \otimes \mathscr{T}_{n}^{l_{i}},
$$

in which both equivalences are the same by commutativity of the multiplicative structure on $\mathscr{T}_{n}$. Similarly, $\left(l_{i}+l_{j}\right)+l_{k}=l=l_{i}+\left(l_{j}+l_{k}\right)$ requires associativity. Since these are the only constraints on the free abelian group $\Lambda_{T}^{k}$, indeed the structure of 
commutative multiplicativity suffices to give consistent trivializations. These are by definition commutative multiplicative trivializations, so that the gluing data is indeed a commutative multiplicative torsor, as claimed.

Suppose that we are given $\mathscr{Z}_{n}$ for all $n$ forming an sf comultiplicative Fact $(T(A))_{n}$-gerbe; we show that the corresponding $A$-gerbes $\mathscr{T}_{n}$ are a multiplicative sf $A$-gerbe by proving the claimed formula for them. Since the change of groups can be applied locally it suffices to prove this recursively for torsors and then functions; in the last case, the statement is merely the second part of Lemma II.4.7 combined with Lemma II.4.2.

Finally, we must show that the constructions invert each other. Since they are given recursively, it suffices to show this for functions, which is exactly the content of Lemma II.4.7

In the wake of the apparently facile reduction of the theorem to the trivial case of functions, it must be noted that although multiplicative $A$-valued locally constant functions on $\mathrm{Gr}_{T, X}$ are, by definition, identified with sections of ${ }^{L} T(A)=$ Fact $\left({ }^{L} T(A)\right)_{1}$, it is not true that multiplicative $A$-gerbes on $\operatorname{Gr}_{T, X}$ are identified with ${ }^{L} T(A)$-gerbes on $X$; the reason is that multiplicativity is an additional structure on an $A$-gerbe that is not reflected in any such structure on the corresponding ${ }^{L} T(A)$-gerbe. (In the above proof, this structure was used in restricting the gluing data of $\mathscr{Z}_{n}$ from being an arbitrary torsor to being sf multiplicative, so that the recursive reduction could apply.) The correct analogue of sf comultiplicativity which turns this incorrect theorem into a correct one is simply the removal of factorizability, as it was removed from the multiplicative $A$-gerbe:

Definition II.4.10. Let $\mathcal{A}$ be a sheaf of abelian groups on $X$, as in Definition II.4.4. Given an $\mathcal{A}$-gerbe $\mathscr{Z}$ on $X$, the structure of comultiplicativity is, denoting by $\psi: \mathcal{A} \rightarrow \mathcal{A} \times \mathcal{A}$ the diagonal map, an equivalence

$$
{ }^{2} \psi(\mathscr{Z}) \cong \mathscr{Z} \times \mathscr{Z}
$$

together with associativity constraints with the natural compatibilities. The further structure of commutativity is the data of an isomorphism of the composition

$$
\mathscr{Z}_{(1)} \times \mathscr{Z}_{(2)} \cong{ }^{2} \psi(\mathscr{Z}) \cong{ }^{2} \mathrm{sw}^{2} \psi(\mathscr{Z}) \cong \mathscr{Z}_{(2)} \times \mathscr{Z}_{(1)},
$$

(where the subscripts denote logical labeling of the factors and we denote by sw : $\mathcal{A} \times \mathcal{A} \rightarrow \mathcal{A} \times \mathcal{A}$ the factor-switching map) with the natural auto-equivalence of $\mathscr{Z} \times \mathscr{Z}$. It should have natural compatibilities with itself and with the associativity constraint. Likewise, we define (commutative) comultiplicative torsors for and sections of $\mathcal{A}$ (the latter of which are, commutative or not, all sections of $\mathcal{A}$ ).

Lemma II.4.11. When $\mathcal{A}=\underline{A}$ is the constant sheaf on an abelian group, if the $\mathscr{Z}_{n}$ form an sf comultiplicative gerbe for $\operatorname{Fact}(A)_{n}$ over $X^{n}$, then for any $x \in X$, the fiber $\mathscr{Z}=\left.\mathscr{Z}_{1}\right|_{x}$ is commutative comultiplicative.

Proof. The map $\psi_{1,1}$ of (II.10) is just the diagonal map $A \rightarrow A \times A$ over any point of the diagonal in $X^{2}$.

In the next proposition, we use the notation ${ }^{L} T(\mathcal{A})=\mathcal{H} \circ \mathrm{om}\left(\Lambda_{T}, \mathcal{A}\right) \cong \Lambda_{T} \otimes \mathcal{A}$, which agrees with the previous definition given in Lemma II.4.5.

Proposition II.4.12. For any sheaf of abelian groups $\mathcal{A}$ on $X$, the constructions of Definition II.4.8, for $n=1$ alone, are inverse equivalences of the 2-categories of 
commutative multiplicative A-gerbes $\mathscr{T}$ on $\mathrm{Gr}_{T, X}$ and commutative comultiplicative ${ }^{L} T(A)$-gerbes $\mathscr{Z}$ on $X$. Given $\mathscr{Z}$ and any $\lambda \in \Lambda_{T}=\operatorname{Hom}\left({ }^{L} T, \mathbf{G}_{\mathbf{m}}\right)$, if we also consider $\lambda \in \operatorname{Hom}\left({ }^{L} T(A), A\right)$ then the corresponding $\mathscr{T}^{\lambda}$ is ${ }^{2} \lambda(\mathscr{Z})$; the multiplicative structure is that obtained from $\mathscr{Z}$.

The proof is exactly the same as before. As a final remark, suppose $\mathscr{F}$ is a sheaf of categories over $\mathrm{Gr}_{T, X}$ with a $\Lambda_{T}$-equivariant pairing as in Definition I.4.4 which is bi-equivariant for an action of $A$-torsors on objects of $\mathscr{F}$. Thus, if $\mathscr{T}$ is a commutative multiplicative gerbe, $\mathscr{F}(\mathscr{T})$ has a twisted pairing.

Alternatively, we consider $\mathscr{F}$ as a sheaf of categories $p_{*} \mathscr{F}$ (writing $p: \operatorname{Gr}_{T, X} \rightarrow$ $X$ ) over $X$ whose objects have $\Lambda_{T}$-gradings and thus ${ }^{L} T$-actions, and which has a pairing which is bi-equivariant for an action of ${ }^{L} T(A)$-torsors. Thus, we may form the twisted category $p_{*} \mathscr{F}(\mathscr{Z})$, where $\mathscr{Z}$ is the commutative comultiplicative ${ }^{L} T(A)$-gerbe corresponding to $\mathscr{T}$. By definition of the product of $\Lambda_{T}$-gradings, the pairing on $p_{*} \mathscr{F}$ can be written as a composition

$$
p_{*} \mathscr{F} \times p_{*} \mathscr{F} \rightarrow \mathscr{F}_{2} \rightarrow p_{*} \mathscr{F},
$$

where $\mathscr{F}_{2}$ consists of $\Lambda_{T}$-bigraded objects; the second map is taking the total grading. In terms of ${ }^{L} T$-actions, it is restriction along the diagonal map $\psi:{ }^{L} T \rightarrow$

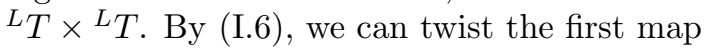

$$
p_{*} \mathscr{F}(\mathscr{Z}) \times p_{*} \mathscr{F}(\mathscr{Z}) \rightarrow \mathscr{F}_{2}(\mathscr{Z} \times \mathscr{Z}) \cong \mathscr{F}_{2}\left({ }^{2} \psi \mathscr{Z}\right) \rightarrow p_{*} \mathscr{F}(\mathscr{Z})
$$

by comultiplicativity of $\mathscr{Z}$. Thus, we obtain a twisted product on $p_{*} \mathscr{F}(\mathscr{Z})$.

The following proposition is obvious from the proof of Proposition II.4.12.

Proposition II.4.13. There is a natural equivalence of sheaves of categories $p_{*} \mathscr{F}(\mathscr{Z})$ and $\mathscr{F}(\mathscr{T})$ identifying their respective pairings.

\section{II.5. THE AFFINE GRASSMANNIAN OF ANY GROUP}

In this section we discuss the relationships among the affine grassmannians of more general algebraic groups and their individual structures.

The grassmannian of a torus. A basic fact about the $\operatorname{Gr}_{G, X^{n}}$ is that their formation is functorial in $G$, in that any regular homomorphism $G \rightarrow H$ induces a map of ind-schemes $\mathrm{Gr}_{G, X^{n}} \rightarrow \mathrm{Gr}_{H, X^{n}}$ constructed by induction of $G$-torsors to $H$-torsors. In particular, if we choose a maximal torus $T \subset G$, then we get an induced map

$$
i: \mathrm{Gr}_{T, X^{n}} \rightarrow \mathrm{Gr}_{G, X^{n}} .
$$

We continue to denote by $\operatorname{Gr}_{T, X^{n}}^{\lambda_{1}, \ldots, \lambda_{n}}$ the images of these components under $i$. Given the description of the $\mathrm{Gr}_{T, X}^{\lambda}$ in Proposition II.1.2 it is easy to see that the $\mathrm{Gr}_{T, X^{n}}^{\lambda_{1}, \ldots, \lambda_{n}}$ can be described explicitly in $\operatorname{Gr}_{G, X^{n}}$ :

Proposition II.5.1. Let $\lambda_{1}, \ldots, \lambda_{n} \in \Lambda_{T}$ be coweights of $G$ and let $p=(\vec{x}, \mathcal{T}, \phi) \in$ $\operatorname{Gr}_{G, X^{n}}(S)$ for some scheme $S$. Then $p \in \operatorname{Gr}_{T, X^{n}}^{\lambda_{1}, \ldots, \lambda_{n}}(S)$ if and only if, for every $G$-representation $V$ and every vector $v \in V$ of some weight $\mu \in \Lambda^{\vee}$, the inclusion over $X_{S} \backslash \bar{x}$,

$$
\left.\langle v\rangle \otimes \mathcal{O}_{X_{S} \backslash \bar{x}} \hookrightarrow V \otimes \mathcal{O}_{X_{S} \backslash \bar{x}} \stackrel{\phi}{\sim} V_{\mathcal{T}}\right|_{X_{S} \backslash \bar{x}},
$$

extends to an inclusion of vector bundles $\mathcal{O}\left(\sum_{i}\left\langle\mu, \lambda_{i}\right\rangle \bar{x}_{i}\right) \rightarrow V_{\mathcal{T}}$. 
The grassmannian of a Borel subgroup. Now say we choose a Borel subgroup $B$, and let $T=B / N$, where $N$ is the unipotent part of $B$; we do not choose an inclusion of $T$ into $G$. Then the pair of maps $B \rightarrow G$ and $B \rightarrow T$ induce a diagram

$$
\operatorname{Gr}_{G, X^{n}} \stackrel{b}{\leftarrow} \operatorname{Gr}_{B, X^{n}} \stackrel{t}{\rightarrow} \operatorname{Gr}_{T, X^{n}}
$$

in which $b$ is surjective (proof: a reduction of a $G$-torsor to $B$ is the same as a trivialization of the induced $G / B$-bundle. This exists away from a divisor by assumption, so by the valuative criterion of properness for $G / B$, it exists globally) and, on each component of $\mathrm{Gr}_{B, X^{n}}$, is injective. Although we do not choose an embedding $T \subset G$, for every splitting of $B \rightarrow T$, the induced map $i$ makes the above diagram commute.

We will require the following description of the components of $\mathrm{Gr}_{G}$ :

\section{Proposition II.5.2. ([MV07])}

(1) Each inverse image $t^{-1}\left(\mathrm{Gr}_{T}^{\lambda}\right)$ is connected, so that the components of $\mathrm{Gr}_{B}$ are also indexed by $\Lambda_{T}$.

(2) There is an embedding of $\mathrm{Gr}_{G}$ in $\mathbb{P}^{\infty}=\lim _{n} \mathbb{P}^{n}$ such that for any Borel subgroup B, each boundary $\partial \operatorname{Gr}_{B}^{\lambda}=\bigcup_{\mu<\lambda} \overrightarrow{G r}_{B}^{\mu}$ is a hyperplane section of $\overline{\mathrm{Gr}}_{B}^{\lambda}$. In fact, $\mathrm{Gr}_{B}^{\lambda} \cong \mathbb{A}^{\infty}$.

(3) In $\mathrm{Gr}_{G}$, we have $\overline{\mathrm{Gr}}_{B}^{\lambda}=\bigcup_{\mu \leq \lambda} \mathrm{Gr}_{B}^{\mu}$.

By the second point, the components $\operatorname{Gr}_{B}^{\lambda}$ contained, via $b$, in each component of $\mathrm{Gr}_{G}$ are indexed by the cosets of the coroot lattice $\Lambda_{T, r}$ of $G$, so that the set of connected components of $\mathrm{Gr}_{G}$ is identified with $\pi_{1}(G)=\Lambda_{T} / \Lambda_{T, r}$. More precisely, each component of $\operatorname{Gr}_{G}$ contains exactly one $\operatorname{Gr}_{T}^{\lambda}$ where $\lambda$ is minimal dominant with respect to the partial ordering by positive coroots. These statements hold identically for $\mathrm{Gr}_{G, X}$, etc. as well.

The grassmannians of subminimal parabolics. The preceding constructions for $B$ and $T$ admit natural generalizations where $B$ is replaced by any parabolic subgroup $P$ containing $T$ and $T$ is replaced by its Levi quotient $L$. Then we have the maps

$$
\mathrm{Gr}_{G, X^{n}} \stackrel{p}{\leftarrow} \operatorname{Gr}_{P, X^{n}} \stackrel{l}{\rightarrow} \operatorname{Gr}_{L, X^{n}}
$$

and for any splitting of $P \rightarrow L$, a map $i$ : $\operatorname{Gr}_{L, X^{n}} \rightarrow \mathrm{Gr}_{G, X^{n}}$. We will apply this construction when $L$ is a Levi subgroup of semisimple rank 1 as follows. Inside of $\mathrm{Gr}_{G, X^{n}}$, the components of $\mathrm{Gr}_{T, X^{n}}$ are connected by projective line bundles with good factorizability properties. We will only need these for $n=1,2$ and will in fact obtain them first when $G$ has semisimple rank 1 and then push them forward along the map $i$ as $L$ varies over all the Levi factors of the subminimal parabolics of $G$.

Definition II.5.3. Let $G=\mathrm{GL}_{2}$. We identify $\Lambda_{T} \cong \mathbb{Z}^{2}$ and the simple coroot $\check{\alpha}=(1,-1)$; let $V$ be the standard representation of $\mathrm{GL}_{2}$, with weights $(1,0)$ and $(0,1)$, and let $V(1,0), V(0,1)$ be its weight spaces. Also, let $\Omega$ be the determinant representation, with weight $(1,1)$. Let $\lambda=\left(\lambda_{1}, \lambda_{2}\right)$ and $\mu=\left(\mu_{1}, \mu_{2}\right)$ be coweights 
and define the following subfunctors of $\operatorname{Gr}_{\mathrm{GL}_{2}, X^{n}}(n=1,2)$ :

$$
\begin{aligned}
\mathbb{P}_{1}^{\lambda}(S)=\left\{\begin{array}{c|c}
(x, \mathcal{T}, \phi) & V(1,0) \otimes \mathcal{O}_{X_{S}} \hookrightarrow V_{\mathcal{T}}\left(-\lambda_{1} \bar{x}\right) \\
\in \mathrm{Gr}_{\mathrm{GL}_{2}, X} & V(0,1) \otimes \mathcal{O}_{X_{S}} \hookrightarrow V_{\mathcal{T}}\left(-\left(\lambda_{2}-1\right) \bar{x}\right) \\
\Omega & \otimes \mathcal{O}_{X_{S}} \hookrightarrow \Omega_{\mathcal{T}}\left(-\left(\lambda_{1}+\lambda_{2}\right) \bar{x}\right)
\end{array}\right\}, \\
\mathbb{P}_{2,1}^{\lambda, \mu}(S)=\left\{\begin{array}{c|c}
(x, y, \mathcal{T}, \phi) & V(1,0) \otimes \mathcal{O}_{X_{S}} \hookrightarrow V_{\mathcal{T}}\left(-\lambda_{1} \bar{x}-\left(\mu_{1}+1\right) \bar{y}\right) \\
\in \mathrm{Gr}_{\mathrm{GL}_{2}, X^{2}} & V(0,1) \otimes \mathcal{O}_{X_{S}} \hookrightarrow V_{\mathcal{T}}\left(-\left(\lambda_{2}-1\right) \bar{x}-\left(\mu_{2}-1\right) \bar{y}\right) \\
\Omega & \otimes \mathcal{O}_{X_{S}} \hookrightarrow \Omega_{\mathcal{T}}\left(-\left(\lambda_{1}+\lambda_{2}\right) \bar{x}-\left(\mu_{1}+\mu_{2}\right) \bar{y}\right)
\end{array}\right\},
\end{aligned}
$$

where each inclusion is an inclusion of coherent sheaves (not vector bundles), and the maps refer, like those in Proposition II.5.1, to the ones given by the trivialization $\phi$ on $X \backslash \bar{x}$ or $X \backslash(\bar{x} \cup \bar{y})$.

We require these subspaces for their excellent properties vis à vis factorization, giving $\mathrm{Gr}_{G, X^{2}}$ some of the flavor of Proposition II.1.2 which it, in general, lacks.

Lemma II.5.4. We have $\mathbb{P}_{2,1}^{\lambda, \mu} \cong \mathbb{P}_{1}^{\lambda} \times \mathrm{Gr}_{T, X}^{\mu+\alpha}$, and this isomorphism agrees with the factorization isomorphism over $X^{2} \backslash \Delta$. Define

$$
\begin{aligned}
U_{1}^{0} & =\mathbb{P}_{1}^{\lambda} \cap \operatorname{Gr}_{B, X}^{\lambda}, & U_{2,1}^{0}=\mathbb{P}_{2,1}^{\lambda, \mu} \cap \operatorname{Gr}_{B, X}^{\lambda, \mu+\check{\alpha}}, \\
U_{1}^{\infty} & =\mathbb{P}_{1}^{\lambda} \cap \operatorname{Gr}_{B^{\mathrm{op}}, X}^{\lambda,}, & U_{2,1}^{\infty}=\mathbb{P}_{2,1}^{\lambda, \mu} \cap \operatorname{Gr}_{B^{\mathrm{op}}, X}^{\lambda, \mu}
\end{aligned}
$$

(where $B^{\text {op }}$ is the Borel subgroup opposite to $B$ ); then $U_{1}^{0} \cong U_{1}^{\infty} \cong \mathbb{A}^{1} \times X$, $U_{2,1}^{0} \cong U_{2,1}^{\infty} \cong \mathbb{A}^{1} \times X^{2}$, and these make $\mathbb{P}_{1}^{\lambda}$ and $\mathbb{P}_{2,1}^{\lambda, \mu}$ into $\mathbb{P}^{1}$-bundles on $X$ and $X^{2}$, respectively. From these formulas, $\mathbb{P}_{1}^{\lambda}$ has $\operatorname{Gr}_{T, X}^{\lambda}$ and $\mathrm{Gr}_{T, X}^{\lambda+\check{\alpha}}$ as zero and infinity sections, respectively, and likewise, $\mathbb{P}_{2,1}^{\lambda, \mu}$ has $\mathrm{Gr}_{T, X^{2}}^{\lambda, \mu+\check{\alpha}}$ and $\mathrm{Gr}_{T, X^{2}}^{\lambda+\check{\alpha}, \mu+\check{\alpha}}$ as zero and infinity sections.

Finally, suppose $\mu$ is a dominant weight, so $\mu_{1}-\mu_{2} \geq 0$. Let

$$
\mathbb{A}^{1} \times\left(X^{2} \backslash \Delta\right) \rightarrow \mathbb{A}^{1} \times\left(X^{2} \backslash \Delta\right)
$$

be the restriction of the factorization map $\mathbb{P}_{1}^{\lambda} \times \mathrm{Gr}_{T, X}^{\mu+\check{\alpha}} \rightarrow \mathbb{P}_{2,1}^{\lambda, \mu}$ to $U_{1}^{0} \times \mathrm{Gr}_{T, X}^{\mu+\check{\alpha}} \rightarrow U_{2,1}^{0}$. Then the corresponding map $\mathbb{A}^{1} \times\left(X^{2} \backslash \Delta\right) \rightarrow \mathbb{A}^{1}$ has a zero of order $\left(\mu_{1}-\mu_{2}\right)+2=$ $\langle\alpha, \mu\rangle+2$ along $\Delta$. Likewise, on $U_{1}^{\infty} \times \operatorname{Gr}_{T, X}^{\mu+\check{\alpha}}$ it has a pole of this order.

Proof. It is obvious that $\mathbb{P}_{2,1}^{\lambda, \mu} \cong \mathbb{P}_{1}^{\lambda} \times \mathrm{Gr}_{T, X}^{\lambda+\check{\alpha}}$, using Proposition II.5.1 as a description of the latter, and from the construction of the factorization isomorphism in the proof of Proposition II.1.3 that this isomorphism agrees with factorization away from $\Delta$. For the rest of the computations, we will compute the $\mathbb{C}$-points and show that they have the desired structure, leaving the generalization to $S$-families to the imagination. We use the fact that a $\mathrm{GL}_{2}$-torsor $\mathcal{T}$ is equivalent to its associated vector bundle $V_{\mathcal{T}}$, with $V$ the standard representation.

We first prove that $\mathbb{P}_{1}^{\lambda}$ has the desired form; namely, we will show that if $p=$ $(x, \mathcal{T}, \phi) \in \mathbb{P}_{1}^{\lambda}(\mathbb{C})$, then in any sufficiently small neighborhood of $x, V_{\mathcal{T}}$ may be trivialized so that $\phi$ is given by one (possibly either) of the following forms, and that this trivialization is unique (for each form):

$$
A_{+}=\left[\begin{array}{cc}
z_{x}^{\lambda_{1}} & s z_{x}^{\lambda_{2}-1} \\
0 & z_{x}^{\lambda_{2}}
\end{array}\right], \quad A_{-}=\left[\begin{array}{cc}
z_{x}^{\lambda_{1}+1} & 0 \\
t z_{x}^{\lambda_{1}} & z_{x}^{\lambda_{2}-1}
\end{array}\right],
$$


for some $s, t \in \mathbb{C}$; here, $z_{x}$ is a generator of the maximal ideal $\mathfrak{m}_{x}$. We pick any trivialization of $V_{\mathcal{T}}$, so that the maps $V(1,0) \otimes \mathcal{O}_{X} \hookrightarrow V_{\mathcal{T}}, V(0,1) \otimes \mathcal{O}_{X} \hookrightarrow V_{\mathcal{T}}(x)$ are given by a matrix with entries in $K(X)$,

$$
A=\left[\begin{array}{ll}
a & b \\
c & d
\end{array}\right]
$$

in which $a, c \in \mathfrak{m}_{x}^{\lambda_{1}}$ and $b, d \in \mathfrak{m}_{x}^{\lambda_{2}-1}$. Two such matrices determine equivalent trivializations $\phi$ if they differ by the action of $\mathrm{GL}_{2}\left(\mathcal{O}_{X}\right)$ on the left, so we may perform row operations with coefficients in $\mathcal{O}_{X}$. Unless both $a, c \in \mathfrak{m}_{x}^{\lambda_{1}+1}$, we may perform row operations to put $a \in \mathfrak{m}_{x}^{\lambda_{1}}$, and then put $A$ in the form

$$
\left[\begin{array}{ll}
a & b \\
0 & d
\end{array}\right]=\left[\begin{array}{cc}
z_{x}^{\lambda_{1}} & s z_{x}^{\lambda_{2}-1} \\
0 & z_{x}^{\lambda_{2}}
\end{array}\right]
$$

where we have applied the determinant condition to find the order of vanishing of $d$ and chosen some regular function $s$ according to $b \in \mathfrak{m}_{x}^{\lambda_{2}-1}$. After one more row operation we may assume $s \in \mathbb{C}$, so $A$ is in the form $A_{+}$. On the contrary, suppose $a, c \in \mathfrak{m}_{x}^{\lambda_{1}+1}$; then we do not have both $b, d \in \mathfrak{m}_{x}^{\lambda_{2}}$ by the determinant condition, so the same logic applies to the second column and, after some row operations, $A$ takes the form in $A_{-}$:

$$
\left[\begin{array}{ll}
a & 0 \\
c & d
\end{array}\right]=\left[\begin{array}{cc}
z_{x}^{\lambda_{1}+1} & 0 \\
t z_{x}^{\lambda_{1}} & z_{x}^{\lambda_{2}-1}
\end{array}\right]
$$

No row operation preserves the forms $A_{+}$or $A_{-}$, so the trivialization inducing them is unique. The points $p$ in which $\phi$ admits the form $A_{+}$are by definition $U_{1}^{0}$ and clearly isomorphic to $\mathbb{A}^{1} \times X$, while those of the form $A_{-}$are $U_{1}^{\infty} \cong \mathbb{A}^{1} \times X$. Clearly, in $U_{1}^{0} \cap U_{1}^{\infty}$, we have the relation $s=t^{-1}$ between their coordinates, so that $U_{1}^{0} \cup U_{1}^{\infty} \cong \mathbb{P}^{1} \times X$.

A similar analysis shows that any point $p=(x, y, \mathcal{T}, \phi)$ in $\mathbb{P}_{2,1}^{\lambda, \mu}$ can, in any sufficiently small neighborhood containing both $x$ and $y$ (if one such exists) and for a unique trivialization of $V_{\mathcal{T}}$, take either of the forms:

$$
A_{+}=\left[\begin{array}{cc}
z_{x}^{\lambda_{1}} z_{y}^{\mu_{1}+1} & s z_{x}^{\lambda_{2}-1} z_{y}^{\mu_{2}-1} \\
0 & z_{x}^{\lambda_{2}} z_{y}^{\mu_{2}-1}
\end{array}\right], \quad A_{-}=\left[\begin{array}{cc}
z_{x}^{\lambda_{1}+1} z_{y}^{\mu_{1}+1} & 0 \\
t z_{x}^{\lambda_{1}} z_{y}^{\mu_{1}+1} & z_{x}^{\lambda_{2}-1} z_{y}^{\mu_{2}-1}
\end{array}\right],
$$

with $s, t \in \mathbb{C}$. As before, choose any trivialization of $V_{\mathcal{T}}$ to bring $\phi$ into the form $A$ as above, with $a, c \in \mathcal{O}_{X}\left(-\lambda_{1} x-\left(\mu_{1}+1\right) y\right)$ and $b, d \in \mathcal{O}_{X}\left(-\left(\lambda_{2}-1\right) x-\left(\mu_{2}-1\right) y\right)$. Suppose we do not have both $a, c \in \mathfrak{m}_{x}^{\lambda_{1}+1}$; by a suitable linear combination (row operation), we may assume that $a \notin \mathfrak{m}_{x}^{\lambda_{1}+1}$ and that $c$ has a higher-order zero at $y$, so that $c / a \in \mathcal{O}_{X}$ in a sufficiently small neighborhood of $x, y$. Thus, row operations bring $A$ into the form

$$
\left[\begin{array}{ll}
a & b \\
0 & d
\end{array}\right]=\left[\begin{array}{cc}
z_{x}^{\lambda_{1}} z_{y}^{\mu_{1}+1} & b \\
0 & z_{x}^{\lambda_{2}} z_{y}^{\mu_{2}-1}
\end{array}\right]
$$

by the determinant condition. Then $b$ must be of the form $s z_{x}^{\lambda_{2}-1} z_{y}^{\mu_{2}-1}$ for some regular function $s$, and further row operations bring $s$ into $\mathbb{C}$, and hence $A$ into the form $A_{+}$. If, conversely, we have both $a, c \in \mathfrak{m}_{x}$, then the same logic applies to $b, d$ by the determinant condition, and row operations bring $A$ into the form $A_{+}$. As above, these matrices are obtained by unique trivializations of $V_{\mathcal{T}}$ and form, respectively, $U_{2,1}^{0}$ and $U_{2,1}^{\infty}$, both isomorphic to $\mathbb{A}^{1} \times X^{2}$, with $s=t^{-1}$ on $U_{2,1}^{0} \cap U_{2,1}^{\infty}$, so that $\mathbb{P}_{2,1}^{\lambda, \mu} \cong \mathbb{P}^{1} \times X^{2}$ (locally). 
We now apply the construction of the factorization isomorphism in the proof of Proposition II.1.3 to compute it explicitly in these coordinates. Let $\left(x, \mathcal{T}_{x}, \phi_{x}\right)$ and $\left(y, \mathcal{T}_{y}, \phi_{y}\right)$ be points in $U_{1}^{0}(\mathbb{C})$ and $\operatorname{Gr}_{T, X}^{\mu+\check{\alpha}}(\mathbb{C})$, and let

$$
\mathcal{T}=\left.\left.\mathcal{T}_{x}\right|_{X \backslash\{y\}} \cup_{X \backslash\{x, y\}} \mathcal{T}_{x}\right|_{X \backslash\{x\}}
$$

be the glued torsor. Suppose we have chosen trivializations of $V_{\mathcal{T}_{x}}$ and $V_{\mathcal{T}_{y}}$ such that $\phi_{x}$ is in the form $A_{+}$given in (II.16), and $\phi_{y}$ is given by the diagonal matrix $\operatorname{diag}\left(z_{y}^{\mu_{1}+1}, z_{y}^{\mu_{2}-1}\right)$; denote their bases $e_{1, x}, e_{2, x}$ and $e_{1, y}, e_{2, y}$. We define a global basis $f_{1}, f_{2}$ of $V_{\mathcal{T}}$ such that:

$$
\begin{array}{ll}
\left.f_{1}\right|_{X \backslash\{y\}}=z_{y}^{-\left(\mu_{1}+1\right)} e_{1, x}, & \left.f_{2}\right|_{X \backslash\{y\}}=z_{y}^{-\left(\mu_{2}+1\right)} e_{2, x}, \\
\left.f_{1}\right|_{X \backslash\{x\}}=z_{x}^{-\lambda_{1}} e_{1, y}, & \left.f_{2}\right|_{X \backslash\{x\}}=-s z_{x}^{-\left(\lambda_{1}+1\right)} z_{y}^{\left(\mu_{1}-\mu_{1}\right)+2} e_{1, y}+z_{x}^{-\lambda_{2}} e_{2, y} .
\end{array}
$$

Let $e_{1}, e_{2}$ be the basis of weight vectors in $V$, so that on $X \backslash\{x, y\}$, accounting for the form of the trivializations $\phi_{x}, \phi_{y}$, we have

$$
\begin{gathered}
{\left[\begin{array}{l}
f_{1} \\
f_{2}
\end{array}\right]=\left[\begin{array}{cc}
z_{x}^{-\lambda_{1}} z_{y}^{-\left(\mu_{1}+1\right)} & -s z_{x}^{-\left(\lambda_{1}+1\right)} z_{y}^{-\left(\mu_{2}+1\right)} \\
0 & z_{x}^{-\lambda_{2}} z_{y}^{-\left(\mu_{2}+1\right)}
\end{array}\right]\left[\begin{array}{l}
e_{1} \\
e_{2}
\end{array}\right],} \\
{\left[\begin{array}{l}
e_{1} \\
e_{2}
\end{array}\right]=\left[\begin{array}{cc}
z_{x}^{\lambda_{1}} z_{y}^{\mu_{1}+1} & s z_{x}^{\lambda_{2}-1} z_{y}^{\mu_{1}+1} \\
0 & z_{x}^{\lambda_{2}} z_{y}^{\mu_{2}+1}
\end{array}\right]\left[\begin{array}{l}
f_{1} \\
f_{2}
\end{array}\right]}
\end{gathered}
$$

Comparing this expression to that for $A_{+}$in (II.17), we find that the $s$ there is given by $s z_{y}^{\mu_{1}-\mu_{2}+2}$. Let $z_{y-x}=z_{y}-z_{x}$ be the uniformizer along the diagonal in $X^{2}$, so $z_{y}-z_{y-x} \in \mathfrak{m}_{x}$. After a row operation, we find that the $\mathbb{A}^{1}$-coordinate in (II.17) has a zero of order $\left(\mu_{1}-\mu_{2}\right)+2$ along $\Delta$, as claimed.

Having constructed the above projective line bundles for $\mathrm{GL}_{2}$, we obtain them in particular for $\mathrm{SL}_{2}$ and $\mathrm{PGL}_{2}$, as we have an inclusion $\mathrm{Gr}_{\mathrm{SL}_{2}, X^{n}} \rightarrow \mathrm{Gr}_{\mathrm{GL}_{2}, X^{n}}$ and a projection $\mathrm{Gr}_{\mathrm{GL}_{2}, X^{n}} \rightarrow \mathrm{Gr}_{\mathrm{PGL}_{2}, X^{n}}$ which are isomorphisms on each component. Then for any group $L$ of semisimple rank 1 , let $\bar{L}$ be its semisimple quotient, isomorphic to either one of these two, and the map $\operatorname{Gr}_{L, X^{n}} \rightarrow \mathrm{Gr}_{\bar{L}, X^{n}}$ is an isomorphism on each component, so we define $\mathbb{P}_{1}^{\lambda}$ and $\mathbb{P}_{2,1}^{\lambda, \mu}$ in $\operatorname{Gr}_{L, X^{n}}$ by transport of structure. Finally, let $G$ be any reductive group, $\check{\alpha}$ any simple coroot, and $L$ the corresponding Levi subgroup of $G$, which has the same torus $T$ and thus the same coweights. The maps $\mathrm{Gr}_{L, X^{n}} \rightarrow \mathrm{Gr}_{G, X^{n}}$ are inclusions on each component of the former, so we may define:

Definition II.5.5. Let $\mathbb{P}_{1 ; \check{\alpha}}^{\lambda} \subset \mathrm{Gr}_{G, X}$ be the image of $\mathbb{P}_{1}^{\lambda}$ under the map $\operatorname{Gr}_{L, X} \rightarrow$ $\operatorname{Gr}_{G, X}$ and, likewise, $\mathbb{P}_{2,1 ; \check{\alpha}}^{\lambda, \mu}$. They have the properties given in Lemma II.5.4.

A common factorizable base. Although $\operatorname{Gr}_{G, X^{n}}$ is given only over $X^{n}$, we can boost its base to a larger factorizable scheme resembling $\mathrm{Gr}_{T, X^{n}}$ more closely. Let $\pi_{1}(G)_{X^{n}}=\pi_{1}(G)_{n}$ be the union of copies of $X^{n}$, indexed by $\pi_{1}(G)^{n}$, and intersecting in the pattern described in Proposition II.1.2 then it has an obvious factorizable structure which, again as in the proposition, agrees with the group structure of $\pi_{1}(G)$. The components of $\mathrm{Gr}_{G, X}$ are naturally indexed by $\pi_{1}(G)$, so $\operatorname{Gr}_{G, X}$ admits a map to $\pi_{1}(G)_{X}$. Using the factorizable structure of each, we obtain maps $\operatorname{Gr}_{G, X^{n}} \rightarrow \pi_{1}(G)_{X^{n}}$. Since $\pi_{1}(G)=\Lambda / \Lambda_{r}$, by the proposition there is also a natural map $\operatorname{Gr}_{T, X^{n}} \rightarrow \pi_{1}(G)_{X^{n}}$. 


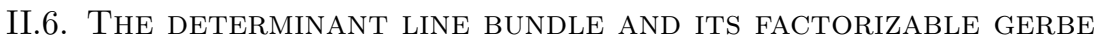

A simpler analogue of sf gerbes is the concept of an sf line bundle on the factorizable grassmannian. The definition is the same as in Definition II.2.1 except with all mention of 2-morphisms omitted. We will use sf line bundles to construct sf gerbes, as follows. Given line bundles $\mathcal{L}_{n}$ on each $\mathrm{Gr}_{n}$, and for $a \in A$, let $\mathscr{G}_{n}=\mathcal{L}_{n}^{\log a}$. By Proposition I.5.4 the factorizable structure of $\mathscr{L}_{n}$ becomes such a structure for $\mathscr{G}_{n}$. The basic such sheaves are the determinant line bundles, obtained as follows.

The determinant line bundle. Given any finite-dimensional representation $V$ of $G$, we will define an associated line bundle $\operatorname{det}(V)_{n}$ on $\mathrm{Gr}_{n}$. Here is the definition; following are supporting lemmas on straightforward facts and sketches of their proofs.

Definition II.6.1. Let $s=(\vec{x}, \mathcal{T}, \phi) \in \operatorname{Gr}_{G, X^{n}}(S)$, and let $V$ be a finite-dimensional representation of $G$. We write $f: \bar{x} \rightarrow S$ for the natural map and $\mathcal{V}=V_{\mathcal{T}}$ for the induced vector bundle. The determinant bundle $\operatorname{det}(V)_{n}$ associated with $V$ is the line bundle on $\operatorname{Gr}_{n}$ whose fiber at $s \in \operatorname{Gr}_{n}(S)$ is any of the following naturally isomorphic line bundles on $S$ :

$$
\left(\operatorname{det}(V)_{n}\right)_{s}= \begin{cases}\bigwedge^{\text {top }} f_{*}(\mathcal{V} / V(a \bar{x})) \otimes\left(\bigwedge^{\text {top }} f_{*}(V(0) / V(a \bar{x}))\right)^{\vee} & \text { any } a \ll 0, \\ \left(\bigwedge^{\text {top }} f_{*}(V(b \bar{x}) / \mathcal{V})\right)^{\vee} \otimes \bigwedge^{\text {top }} f_{*}(V(b \bar{x}) / V(0)) & \text { any } b \gg 0 .\end{cases}
$$

The following lemmas support the claims implicit in this definition.

Lemma II.6.2. Let $j: X_{S} \backslash \bar{x} \rightarrow X_{S}$ be the open immersion. Then there is an inclusion

$$
\mathcal{V} \hookrightarrow j_{*}\left(\mathcal{O}_{U} \otimes V\right)=j_{*} j^{*} V(0)
$$

satisfying the asymptotic containments $V(a \bar{x}) \subset \mathcal{V} \subset V(b \bar{x})$ for $a \ll 0$ and $b \gg 0$.

Sketch of Proof. The map of the inclusion comes by adjunction from the isomorphism induced by the trivialization $\phi$ :

$$
j^{*} \mathcal{V} \cong \mathcal{O}_{U} \otimes V
$$

and it is an injection because $\mathcal{V}$ is torsion-free. The right-hand side contains all the fractional ideal sheaves $V(c \bar{x})$ and the second inclusion follows (after twisting by some irrelevant very ample line bundle) by counting poles of generators of $\mathcal{V}$. The first inclusion is obtained by analyzing any subspace $W \subset V$ for which $W(a \bar{x}) \subset \mathcal{V}$; given any $v \in V$, an application of the second inclusion to $\mathcal{V} \cap j_{*} j^{*}\langle v\rangle(0)$ shows that it can be enlarged to $W+\langle v\rangle$.

Lemma II.6.3. The map $f$ is flat, and for any $a, b$ as in Lemma II.6.2, the quotients $\mathcal{V} / V(a \bar{x})$ and $V(b \bar{x}) / \mathcal{V}$ are $f$-flat.

Sketch of Proof. Flatness of $f$ follows from the fact that it is surjective with fibers of constant finite length. Flatness of the first quotient follows from comparison of two flat resolutions (where $c=a-b$ ):

$$
\begin{aligned}
\cdots \rightarrow \mathcal{V}(c \bar{x}) / \mathcal{V}(2 c \bar{x}) & \rightarrow V(a \bar{x}) / V((a+c) \bar{x}) \rightarrow \mathcal{V} / \mathcal{V}(c \bar{x}) \rightarrow \mathcal{V} / V(a \bar{x}) \rightarrow 0, \\
0 & \rightarrow V(a \bar{x}) \rightarrow \mathcal{V} \rightarrow \mathcal{V} / V(a \bar{x}) \rightarrow 0,
\end{aligned}
$$

of which the first shows that $\operatorname{Tor}_{S}^{i}(\mathcal{V} / V(a \bar{x}), \bullet)$ is (essentially) periodic and the second shows that it is bounded. The same argument works for the second quotient. 
Corollary II.6.4. $f_{*}(\mathcal{V} / V(a \bar{x}))$ and $f_{*}(V(b \bar{x}) / \mathcal{V})$ are vector bundles on $S$.

Proof. Note that both $\mathcal{V} / V(a \bar{x})$ and $V(b \bar{x}) / \mathcal{V}$ are coherent, $f$-flat, and their fibers are flasque (since they live on finite sets), hence acyclic. Since the Euler characteristic is constant in flat families, the pushforwards have constant rank and are thus vector bundles.

It is clear that $\operatorname{det}(V)_{n}$ does not depend on the choice of $a$ or $b$ in Definition II.6.1. Note that $\operatorname{det}(V)_{n}$ is a $\mathbb{Z} /(2)$-graded line bundle; i.e., it caries a parity depending on those of the two "top"s, which is the source of the sign in the following theorem:

Proposition II.6.5. The determinant line bundles $\operatorname{det}(V)_{n}$ satisfy properties Definition II.2.1(1, 2), and possess the equivariance of (3), The compatibility of this equivariance with the natural one given by the tensor product of [2) holds up to a sign which is constant on components of $\mathrm{Gr}_{n}$ over $X^{n}$.

Proof. For property (1) this is trivial, since the vector bundle $\mathcal{V}$ and its inclusion $j^{*} \mathcal{V} \rightarrow \mathcal{O}$ do not depend on the multiplicity of the components of the divisor $\bar{x}$; likewise, the $S_{n}$-symmetry of (3) comes from the fact that these data do not depend on the order of the components of $D$. For property $(2)$, we have $\bar{x}=\bigcup \bar{x}_{i}$, where $\bar{x}_{i}$ is the union of the graphs of the points in the $X^{n_{i}} ;$ let $\mathcal{V}_{i}$ be the vector bundles corresponding to these points, with trivializations on $X_{S} \backslash \bar{x}_{i}$. Then we have the map

$$
\mathcal{V} / V(a \bar{x}) \rightarrow \bigoplus \mathcal{V}_{i} / V\left(a \bar{x}_{i}\right)
$$

for $a \ll 0$, which is an isomorphism away from the intersections of the $D_{i}$. Thus, on $S$ away from these intersections, we have the isomorphism

$$
\bigwedge^{\text {top }} f_{*}(\mathcal{V} / V(a \bar{x})) \cong \bigotimes \bigwedge^{\text {top }} f_{*}\left(\mathcal{V}_{i} / V\left(a \bar{x}_{i}\right)\right)
$$

and, likewise, for the other factor, giving $\operatorname{det}_{n} \cong \bigotimes_{i} \operatorname{det}_{n_{i}}$, as desired. Since the commutativity of this tensor product introduces a sign on the left-hand side, we find that the equivariance of the right-hand side differs from that on the left by this sign, which is constant on connected components of $S$, as claimed.

Computation of the determinant gerbes. Because the determinant line bundle is not quite an sf line bundle, we are not necessarily able to associate an sf gerbe $\operatorname{det}(V)_{n}^{\log a}$ with it. We proceed to investigate the exact identity of the sign in the above theorem so as to specify when this is possible.

It is clear from the definition that if we have a map of groups $G \rightarrow H$ inducing a map of factorizable grassmannians $g: \operatorname{Gr}_{G, X^{n}} \rightarrow \operatorname{Gr}_{H, X^{n}}$, then for any $H$-representation $V_{H}$ considered as a $G$-representation $V_{G}$, we have $\operatorname{det}\left(V_{H}\right)_{n} \cong$ $g^{*} \operatorname{det}\left(V_{G}\right)_{n}$, and so this is true of the associated gerbes as well. On this basis, we compute the sign; as in the definition of $Q\left(\mathscr{G}_{n}\right)$, we begin with the case when $G=T$ is a torus. For the remainder of this section, we change our notation: elements of $\Lambda_{T}=X_{*}(T)$ (coweights) are denoted $\check{\lambda}$, and elements of $\Lambda^{T}=X^{*}(T)$ (weights) are denoted $\lambda$.

Lemma II.6.6. Let $V=\lambda$ be a character representation of $T$. Then for any $\check{\mu}$, if $m=\langle\lambda, \breve{\mu}\rangle$, the determinant bundle on $\operatorname{Gr}_{T, X}$ has component on $\operatorname{Gr}_{T, X}^{\check{\mu}}$ :

$$
\operatorname{det}(V)_{1}^{\check{\mu}}=\mathcal{T}_{X}^{\left(m^{2} / 2\right)-(m / 2)},
$$


where $\mathcal{T}_{X}$ is the tangent bundle on $X$. Furthermore, $\operatorname{det}(V)$ is an sf line bundle if and only if $m$ is even.

Proof. We compute $\operatorname{det}(V)_{1}$ as a line bundle on $S$ for the $S$-point of $\mathrm{Gr}_{T, X}$ corresponding to $S=X$ with the identity map to $X$ and a $T$-torsor $\mathcal{T}$ plus trivialization $\mathcal{T}^{0} \rightarrow \mathcal{T}$ on $X^{2} \backslash \Delta$ making a point of $\mathrm{Gr}_{T, X}^{\breve{\mu}}$. By Proposition II.1.2(1), the corresponding vector bundle is

$$
\mathcal{V}=\mathcal{O}(-m \Delta)
$$

This already implies the last claim of the lemma: comparing with the construction in Proposition II.6.5, we see that the $a$ there is equal to $m$. Since the sign arises from having to move odd-sized blocks past each other in an alternating power, it is equal to 1 if and only if the blocks, which have size $m$, have even size.

Note that also by definition, the sheaf of differentials $\omega_{X}$ is $i_{\Delta}^{*} \mathcal{O}(-\Delta) / \mathcal{O}(-2 \Delta)$ and is a line bundle, so that

$$
\omega_{X}^{d}=i_{\Delta}^{*} \mathcal{O}(-d \Delta) / \mathcal{O}(-(d+1) \Delta)
$$

(for both positive and negative values of $d$; this of course is specific to one-dimensional $X)$. By definition of $\operatorname{det}(V)$, when $m>0$, it is $\bigwedge^{\text {top }}\left(\operatorname{pr}_{X}\right)_{*}(\mathcal{O}(0) / \mathcal{O}(-m \Delta))^{\vee}$, where the argument is a chained extension of the $\omega_{X}^{k}$ for $k=0, \ldots, m-1$. If $m \leq 0$, the quotient is turned around and dualized, but by the above equation, the powers appearing follow the same pattern, the only difference being that the sequence is for $k \in[1, m]$. Thus, after some arithmetic, $\operatorname{det}(V)_{1}^{\check{\mu}}=\mathcal{T}_{X}^{m(m-1) / 2}$ in both cases.

Lemma II.6.7. Let $V$ be a character representation as before. On $\mathrm{Gr}_{2}$, the factorization isomorphism $\operatorname{det}(V)_{2} \cong \operatorname{det}(V)_{1} \otimes \operatorname{det}(V)_{1}$ over $X^{2} \backslash \Delta$ has, on the component $\mathrm{Gr}_{2}^{\breve{\mu}, \breve{\nu}}$, a pole of order $m n$, where $n=\langle\lambda, \breve{\nu}\rangle$.

Proof. As in the previous proof, we $\operatorname{define} \operatorname{det}(V)_{2}$ via the point $X^{2} \rightarrow \mathrm{Gr}_{2}$ corresponding to the component $\operatorname{Gr}_{2}^{\breve{\mu}, \check{\nu}}$ having the data $\left(\left(\mathrm{pr}_{1}, \mathrm{pr}_{2}\right), \mathcal{O}\left(-m \Delta_{12}-\right.\right.$ $\left.n \Delta_{13}\right)$, nat.), where nat. is the natural trivialization away from the graphs $\Delta_{12}$ and $\Delta_{13}$ of the two projections $X^{2} \rightarrow X$. Assuming for simplicity that $m, n>0$, we have a natural map

$$
\mathcal{O} / \mathcal{O}\left(-m \Delta_{12}-n \Delta_{13}\right) \rightarrow \mathcal{O} / \mathcal{O}\left(-m \Delta_{12}\right) \oplus \mathcal{O} / \mathcal{O}\left(-n \Delta_{13}\right)
$$

which away from $\Delta_{12} \cap \Delta_{13}$ is the isomorphism from which the factorization isomorphism is obtained. To compute the desired pole, it suffices to consider (II.18) locally, so we assume that $X$ has a coordinate $x$ and that all the vector bundles are trivial. Then $X^{3}$ has coordinates $(x, y, z)$ and we choose for each of the above sheaves the following bases as $\mathcal{O}_{X^{2} \text {-modules: }}$

$$
(x-y)^{i},(x-y)^{m}(x-z)^{j}, \quad(x-y)^{i}, \quad(x-z)^{j},
$$

where $0 \leq i<m, 1 \leq j<n$. Then (II.18) has the following matrix:

$$
\left(\begin{array}{c|c}
\operatorname{id}_{m \times m} & * \\
\hline 0 & {\left[\left((x-y)^{m}(x-z)^{j} \bmod (x-z)^{n}\right)\left[(x-z)^{k}\right]\right]_{j, k}}
\end{array}\right) .
$$

where the notation $\left[(x-z)^{k}\right]$ means the coefficient of this monomial. The order of the zero at $\Delta$ of the determinant of this matrix is the desired order of the pole of the factorization map. Only the lower-right corner needs to be computed, and it is clear that its determinant is that of the action of $(x-y)^{m}$ acting on $\mathcal{O} / \mathcal{O}\left(-n \Delta_{13}\right)$. 
Since $x-y=(x-z)+(z-y)$, where the former is nilpotent and the latter scalar, this determinant is $(z-y)^{m n}$, as claimed.

Now we may turn to $\operatorname{det}(V)_{n}$, where $V$ is any representation of any reductive group $G$. The above computations immediately imply the following proposition:

Proposition II.6.8. Let $G$ be a reductive group with torus $T$ and let $V$ be a representation of $G$ with weights $\lambda$ (so $V_{T}=\bigoplus \lambda$ is the direct sum of character representations). Define the $\mathbb{Z}$-valued bilinear form on $\Lambda_{T}$,

$$
K(\check{\mu}, \check{\nu})=\sum_{\lambda}\langle\lambda, \check{\mu}\rangle\langle\lambda, \check{\nu}\rangle
$$

then $\operatorname{det}(V)_{n}$ is an sf line bundle if and only if $K$ has values in $2 \mathbb{Z}$, and if so, then the corresponding sf gerbe $\operatorname{det}(V)_{n}^{\log a}$ (for any $a \in A$ ) has associated quadratic form given by

$$
\log _{a} Q(\check{\mu})=R(\check{\mu})=\frac{1}{2} \sum_{\lambda}\langle\lambda, \check{\mu}\rangle^{2} .
$$

Furthermore, if this holds, then the half-weight $\zeta=\frac{1}{2} \sum_{\lambda} \lambda$ is in fact integral, and we have on $\mathrm{Gr}_{T, X}$ :

$$
\operatorname{det}\left(V_{T}\right)_{1}^{\check{\mu}}=\omega_{X}^{\langle\zeta, \breve{\mu}\rangle} \otimes \mathcal{T}_{X}^{R(\check{\mu})} .
$$

That is, in reference to the split exact sequence of Proposition II.3.6, the sf gerbe $\operatorname{det}\left(V_{T}\right)_{n}^{\log a}$ corresponds to the quadratic form $Q$ and the multiplicative gerbe $\omega_{X}^{\log a^{\langle\zeta, \breve{\mu}\rangle}}$.

Proof. The appearance of $\zeta$ is due to Lemma II.6.6, after combining all the weight spaces of the $G$-representation $V$. The only further argument needed is that since the sign in Proposition II.6.5 is constant on connected components and compatible with change of group, we may compute it on the copy of $\operatorname{Gr}_{T, X^{n}} \subset \operatorname{Gr}_{G, X^{n}}$, in which case the previous lemmas apply.

When $G$ is a reductive group of semisimple rank at least 1, then the adjoint representation always satisfies the conditions of the theorem, since its nonzero weights are the positive and negative roots. When $G$ is a torus, this representation is trivial.

\section{II.7. Classification: the General CASE}

In this section we pursue the generalization of Proposition II.3.6 to arbitrary sf gerbes. In order to properly analyze the quadratic form, we need to invoke results belonging thematically to Part III, but to preserve the unity of this part we do so as forward-references, being careful to avoid circularity.

Ultimately, we will need to restrict to quadratic forms induced from $W$-invariant forms with values in $\mathbb{Z}$, and to begin, we investigate equivalent characterizations of them.

Lemma II.7.1. Let $Q: \Lambda_{T} \rightarrow k^{*}$ be a $W$-invariant quadratic form with associated bilinear form $\kappa$. For any coroot $\check{\alpha}$ of $G$, there is a homomorphism $\varepsilon_{\check{\alpha}}: \Lambda_{T} \rightarrow A_{2}$ (the 2-torsion in A) such that

$$
\kappa(\check{\alpha}, \lambda)=\varepsilon_{\check{\alpha}}(\lambda) Q(\check{\alpha})^{\langle\alpha, \lambda\rangle},
$$

which is trivial if $\alpha$ is not twice a weight in $\Lambda^{T}$. 
Proof. Fix $\lambda_{0}$ and denote $k=\left\langle\alpha, \lambda_{0}\right\rangle$. Then by $W$-invariance of $Q$, we have

$$
\begin{aligned}
\kappa\left(-k \check{\alpha}, \lambda_{0}\right) & =Q\left(-k \check{\alpha}+\lambda_{0}\right) Q(-k \check{\alpha})^{-1} Q\left(\lambda_{0}\right)^{-1} \\
& =Q\left(s_{\check{\alpha}} \lambda_{0}\right) Q(\check{\alpha})^{-k^{2}} Q\left(\lambda_{0}\right)^{-1}=Q(\check{\alpha})^{-k^{2}}=Q(\check{\alpha})^{-k\left\langle\alpha, \lambda_{0}\right\rangle} .
\end{aligned}
$$

For any $\lambda$ with $\langle\alpha, \lambda\rangle=n k$, we can write inductively,

$$
\begin{aligned}
\kappa(-k \alpha, \lambda) & =\kappa\left(-k \alpha, \lambda-\lambda_{0}\right) \kappa\left(-k \alpha, \lambda_{0}\right) \\
& =Q(\check{\alpha})^{-k\left\langle\alpha, \lambda-\lambda_{0}\right\rangle} Q(\check{\alpha})^{-k\left\langle\alpha, \lambda_{0}\right\rangle}=Q(\check{\alpha})^{-k\langle\alpha, \lambda\rangle} .
\end{aligned}
$$

If $\langle\alpha, \lambda\rangle=-n k$, then we can replace $\lambda-\lambda_{0}$ with $\lambda+\lambda_{0}$ and conclude the same; thus, the above equality holds for all $n$.

For any coroot $\check{\alpha}$, it is possible to choose $\lambda_{0}$ with $k=-2$, and if $\alpha$ is twice a weight, then in fact every $\lambda$ is of the form considered above for some $n$; otherwise, we can take $k=-1$. Thus, in all cases we have

$$
\kappa(\check{\alpha}, \lambda)^{2} Q(\check{\alpha})^{-2\langle\alpha, \lambda\rangle}=1 ;
$$

therefore $\varepsilon_{\check{\alpha}}$ indeed has values $A_{2}$ and is trivial if $k=-1$ is possible, as claimed.

The homomorphisms $\varepsilon_{\check{\alpha}}$ are but a small manifestation of a larger, more irritating phenomenon. The next lemma describes how to avert it:

Lemma II.7.2. Let $Q \in Q\left(\Lambda_{T}, A\right)^{W}$ be any $W$-invariant form. Then it can be represented as the product of quadratic forms of either of the two following kinds:

- Products of Killing forms, namely $a^{Q_{i}}$, where $a \in B$ for some extension $A \subset B$ and the $Q_{i}: \Lambda_{T} \rightarrow \mathbb{Z}$ are the quadratic forms $Q_{i}(\lambda)=\frac{1}{2} \sum_{\beta}\langle\beta, \lambda\rangle^{2}$ appearing in Proposition II.6.8, where $\beta$ runs over the roots in an irreducible component (indexed by $i$ ) of the root system of $G$.

- Denoting $\Lambda_{T, r}^{\mathbb{Q}}=\Lambda_{T} \cap \mathbb{Q} \Lambda_{T, r} \subset \mathbb{Q} \otimes \Lambda_{T}$, a single form $Q$ with associated bilinear form $\kappa$ for which there exists an integer $k$ such that $Q^{k}\left(\Lambda_{T, r}^{\mathbb{Q}}\right)=1$ and $\kappa^{k}\left(\Lambda_{T, r}^{\mathbb{Q}}, \Lambda_{T}\right)=1$.

$A$ quadratic form $Q$ has $k=1$ in the above expansion if and only if there is an extension $A \subset B$ such that $Q$ lies in the image of $Q\left(\Lambda_{T}, \mathbb{Z}\right)^{W} \otimes B \rightarrow Q\left(\Lambda_{T}, B\right)$.

Proof. The values of $Q$ on any element of $\Lambda_{T, r}$ are determined by the $Q(\check{\alpha})$ and $\kappa(\check{\alpha}, \check{\beta})$ for coroots $\check{\alpha}, \check{\beta}$, and if $Q \in Q\left(\Lambda_{T}, A\right)^{W}$, then the values $Q(\check{\alpha})$ are constant on $W$-orbits among coroots. By Lemma II.7.1, these values of $\kappa$ are also determined, up to 2-torsion, by the values of $Q(\check{\alpha})$, so that $Q^{2}$ is completely determined by the values of $Q$ on $W$-orbits of coroots. In each irreducible component, there are at most two such orbits, the long and short coroots, of which the former are linear combinations of the latter. Thus, $Q^{2}$ is determined by the single values $Q(\check{\alpha})$, where $\check{\alpha}$ runs over representatives of the short roots in the components of the coroot system.

We pick some such coroot $\check{\alpha}_{i}$ in the $i$ th component of the coroot system of $G$ and let $a_{i}=Q\left(\check{\alpha}_{i}\right)$ and $m=Q_{i}\left(\check{\alpha}_{i}\right)$. If $a_{i}$ is not an $m$ th power in $A$, we adjoin an $m$ th root in an extension $B$; replace $a_{i}$ by such a root, so that by the above, $\left(a_{i}^{Q_{i}}\right)^{2}$ takes the values $Q^{2}(\check{\alpha})$ for any $\check{\alpha}$ in the $i$ th component of the coroot system, and is trivial for any other $\check{\alpha}$. Thus, the square of the form

$$
R=Q / \prod_{i} a_{i}^{Q_{i}}
$$


vanishes on the coroot lattice $\Lambda_{T, r}$; by Lemma II.7.1, the square, $\kappa_{R}\left(\Lambda_{T, r}, \Lambda_{T}\right)^{2}$, of its bilinear form also vanishes. Choose $k_{0}$ such that $k_{0} \Lambda_{T, r}^{\mathbb{Q}} \subset \Lambda_{T, r}$, so that we have

$$
\kappa_{R}\left(\Lambda_{T, r}^{\mathbb{Q}}, \Lambda_{T}\right)^{2 k_{0}}=\kappa_{R}\left(k_{0} \Lambda_{T, r}^{\mathbb{Q}}, \Lambda_{T}\right)^{2}=\kappa_{R}\left(\Lambda_{T, r}, \Lambda_{T}\right)^{2}=1 .
$$

Thus, the corresponding power of the quadratic form, $R^{2 k_{0}}$, is a homomorphism on $\Lambda_{T, r}^{\mathbb{Q}} / \Lambda_{T, r}$, making its $k_{0}$ th power trivial, so we may take $k=2 k_{0}^{2}$.

Every Killing form is, by definition, defined by a $W$-invariant integer-valued form, namely $Q_{i}$. For the other case, suppose $Q\left(\Lambda_{T, r}^{\mathbb{Q}}\right)=1$ and $\kappa\left(\Lambda_{T, r}^{\mathbb{Q}}, \Lambda_{T}\right)=1$; then $Q$ and $\kappa$ descend to $\Lambda_{T} / \Lambda_{T, r}^{\mathbb{Q}}$, which is a finitely-generated free abelian group. If we denote by $x_{1}, \ldots, x_{r}$ some basis, then $\kappa$ is determined by its values $a_{i j}=\kappa\left(x_{i}, x_{j}\right)$ :

$$
\kappa\left(\sum_{i} n_{i} x_{i}, \sum_{j} m_{j} x_{j}\right)=\prod_{i, j} a_{i j}^{n_{i} m_{j}} .
$$

The $n_{i}$ and $m_{j}$ are integers, so this expression defines $\kappa$ over $\mathbb{Z}$. By definition of the bilinear form, we have

$$
Q\left(\sum_{i} n_{i} x_{i}\right)=\prod_{i} Q\left(n_{i} x_{i}\right) \prod_{j, k} \kappa\left(x_{j}, x_{k}\right)^{n_{j} n_{k}}
$$

where we have, inductively,

$$
Q((n+1) x)=Q(n x) Q(x) \kappa(x, x)^{n}=Q(x)^{n+1} \kappa(x, x)^{\left(\begin{array}{c}
n+1 \\
2
\end{array}\right),}
$$

thus defining $Q$ over $\mathbb{Z}$ as well.

Conversely, suppose we have a $W$-invariant $\mathbb{Z}$-valued form $Q_{0}$, and apply the decomposition of the first part of the lemma. Since $\mathbb{Z}$ is torsion-free, we have $k=1$, as desired.

We can now state the main theorem of this part; here $\mathbf{H}_{\mathrm{sf}}^{2}(G, X, A)$ means, as before, the 2-category of sf $A$-gerbes on $\mathrm{Gr}_{G, X^{n}}$.

Theorem II.7.3. Let $Q\left(\Lambda_{T}, A\right)_{\mathbb{Z}}^{W} \subset Q\left(\Lambda_{T}, A\right)^{W}$ be the subgroup of $W$-invariant quadratic forms $Q$ coming from $\mathbb{Z}$ in the sense of Lemma II.7.2. Then there is a split short exact sequence of 2-Picard categories:

$$
1 \rightarrow \operatorname{Hom}\left(\pi_{1}(G), \mathbf{H}^{2}(X, A)\right) \rightarrow \mathbf{H}_{s f}^{2}(G, X, A) \rightarrow Q\left(\Lambda_{T}, A\right)_{\mathbb{Z}}^{W} \rightarrow 1 .
$$

The proof occupies the remainder of this section. We begin by defining a 2 functor using (II.14),

$$
F: \mathbf{H}_{\mathrm{sf}}^{2}(G, X, A) \rightarrow \mathbf{H}_{\mathrm{sf}}^{2}(T, X, A), \quad t^{*} F\left(\mathscr{G}_{n}\right)=b^{*} \mathscr{G}_{n}
$$

where the descent is possible because, fixing any inclusion $T \rightarrow G$, we have $\mathscr{T}_{n}=$ $i^{*} \mathscr{G}_{n}$ since (II.13) is a section of (II.14) Using Proposition II.3.6, the theorem then becomes:

Theorem II.7.4. An sf gerbe $\mathscr{T}_{n}$ on $\mathrm{Gr}_{T, X^{n}}$ is of the form $F\left(\mathscr{G}_{n}\right)$ if and only if its associated quadratic form is in $Q\left(\Lambda_{T}, A\right)_{\mathbb{Z}}^{W}$ and its associated multiplicative gerbe descends to $\pi_{1}(G)$ (the quotient of $\Lambda_{T}$ by the coroot lattice $\Lambda_{T, r}$ ). (Recall the notion of a multiplicative gerbe on a group scheme, here the discrete groups $\pi_{1}(G)$ or $\Lambda_{T}$, from Definition I.3.2.)

We will refer to the quadratic form $Q\left(F\left(\mathscr{G}_{n}\right)\right)$ as just $Q\left(\mathscr{G}_{n}\right)$ for an sf gerbe on $\operatorname{Gr}_{G, X}$. The above theorem thus consists of four independent propositions, which we pursue in turn. 
Proposition II.7.5. For any $\pi_{1}(G)$-multiplicative gerbe $\mathscr{G}^{\lambda}\left(\lambda \in \pi_{1}(G)\right)$ and any quadratic form $Q$ on $\Lambda_{T} / \Lambda_{T, r}^{\mathbb{Q}}$, there is an sf gerbe $\mathscr{G}_{n}$ on $\operatorname{Gr}_{G, X}$ lifting that on $\mathrm{Gr}_{T, X^{n}}$ which is associated (via Proposition II.3.6) with the pullback of these data to $\Lambda_{T}$.

Proof. Let $Z$ be the torus with $X_{*}(Z)=\Lambda_{T} / \Lambda_{T, r}^{\mathbb{Q}}$ (note that this is a free abelian group of finite rank); let $q_{Z}: \mathrm{Gr}_{T, X^{n}} \rightarrow \mathrm{Gr}_{Z, X^{n}}$ be the map induced by the quotient $T \rightarrow Z$ and let $q: \pi_{1}(G)_{X^{n}} \rightarrow \mathrm{Gr}_{Z, X^{n}}$, induced by the map $\pi_{1}(G)=\Lambda_{T} / \Lambda_{T, r} \rightarrow$ $\Lambda_{T} / \Lambda_{T, r}^{\mathbb{Q}}$. Finally, let $p: \operatorname{Gr}_{G, X^{n}} \rightarrow \pi_{1}(G)_{X^{n}}$ be the map introduced at the end of Section II.5.

By Proposition II.3.6, there is an sf gerbe $\mathscr{T}_{n}$ on $\mathrm{Gr}_{Z, X^{n}}$ with quadratic form $Q$ (and trivial multiplicative part), and thus $q_{Z}^{*} \mathscr{T}_{n}$ is an sf gerbe on $\mathrm{Gr}_{T, X^{n}}$ with form $Q$; then $\mathscr{G}_{n}=p^{*} q^{*} \mathscr{T}_{n}$ is an sf gerbe on $\mathrm{Gr}_{G, X^{n}}$ whose associated gerbe on $\mathrm{Gr}_{T, X^{n}}$ is $q_{Z}^{*} \mathscr{T}_{n}$, as desired.

Similarly, we can construct an sf gerbe $\mathscr{T}_{n}$ (with trivial quadratic form) directly on $\mathrm{Gr}_{T, X^{n}}$ with multiplicative part $\mathscr{G}^{\lambda}$, considered as a $\Lambda_{T}$-multiplicative gerbe, which descends to $\pi_{1}(G)_{X^{n}}$ since $\mathscr{G}^{\lambda}$ does. Then the pullback of this descended gerbe along $p$ is the desired sf gerbe on $\mathrm{Gr}_{G, X^{n}}$.

All of the remaining claims rely on the following key technical lemma:

Lemma II.7.6. The 2-functor $F: \mathbf{H}_{s f}^{2}(G, X, A) \rightarrow \mathbf{H}_{s f}^{2}(T, X, A)$ given above is faithful. This means: for an sf-gerbe $\mathscr{G}_{n}$ on $\mathrm{Gr}_{G, X^{n}}$, any trivialization of $F\left(\mathscr{G}_{n}\right)$ gives a trivialization of $\mathscr{G}_{n}$; for an automorphism $\phi$ of the trivial sf gerbe, a trivialization of $F(\phi)$ gives one of $\phi$; for a 2-automorphism a of id, if $F(a)=\mathrm{id}$, then $a=\mathrm{id}$.

Furthermore, $F$ identifies $\operatorname{Aut}\left(\mathscr{G}_{n}^{0}\right) \cong \operatorname{Hom}\left(\pi_{1}(G), \mathbf{H}^{1}(X, A)\right)$, where $\mathscr{G}_{n}^{0}$ is the trivial sf gerbe.

The essential part of the proof of this lemma is an analysis of the gerbe $\mathscr{G}$ on $\mathrm{Gr}_{G}$ obtained as the fiber of $\mathscr{G}_{1}$ over any point of $X$. Consider the stratification of $\mathrm{Gr}_{G}$ by the closed sets $\overline{\mathrm{Gr}}_{B}^{\lambda}$, using Proposition II.5.2. The interior of this set is $\operatorname{Gr}_{B}^{\lambda} \cong \mathbb{A}^{\infty}$, and therefore is cohomologically trivial, so that $\mathscr{G}$ has a unique trivialization there. The boundary of this set is the union over the simple coroots $\check{\alpha}$ of the $\overline{\mathrm{Gr}}_{B}^{\lambda-\check{\alpha}}$, and these are all Cartier divisors, so that for each $\check{\alpha}$ the unique trivialization has an associated order $m(\lambda, \check{\alpha})$ about these boundary components.

Lemma II.7.7. The numbers $m(\lambda, \check{\alpha})$ are independent of $\lambda$. If $m(\check{\alpha})$ is their common value, then we have $m(\check{\alpha})=Q(\check{\alpha})^{-1}$ for any simple coroot $\check{\alpha}$, where as before, $Q=Q\left(\mathscr{G}_{n}\right)$ is the quadratic form associated to the full factorizable gerbe.

Proof. Let $\lambda, \mu \in \Lambda$ be arbitrary coweights and consider the projective line bundles $\mathbb{P}_{1 ; \check{\alpha}}^{\lambda}$ and $\mathbb{P}_{2,1 ; \check{\alpha}}^{\lambda, \mu}$ given in Definition II.5.5. We view $\mathbb{P}_{1 ; \check{\alpha}}^{\lambda}$ as a $\mathbb{P}^{1}$-bundle on $\operatorname{Gr}_{T, X}^{\lambda+\check{\alpha}}$, the $\infty$-section, and denote the structure map by $p_{1}$; since $U_{1}^{\infty}$ is an $\mathbb{A}^{1}$-bundle, the restriction of $\mathscr{G}_{1}$ to it is canonically equivalent to the pullback of its restriction to the $\infty$-section, so

$$
\mathscr{G}_{1} \mid \mathbb{P}_{1 ; \check{\alpha}}^{\lambda} \cong p_{1}^{*} \mathscr{T}_{1}^{\lambda+\check{\alpha}} \otimes \mathcal{O}\left(\operatorname{Gr}_{T, X}^{\lambda}\right)^{\log m(\lambda, \check{\alpha})}
$$

by definition of this number. To show that $m(\lambda, \check{\alpha})$ is independent of $\lambda$, we do the same on $\mathbb{P}_{2,1 ; \check{\alpha}}^{\lambda, \mu}$ and apply factorizability on $\Delta$. Since $\mathbb{P}_{2,1 ; \check{\alpha}}^{\lambda, \mu}$ is a $\mathbb{P}^{1}$-bundle on 
$\mathrm{Gr}_{T, X^{2}}^{\lambda+\check{\alpha}, \mu+\check{\alpha}}$ (with bundle map $p_{2}$ ), by the same reasoning we have

$$
\mathscr{G}_{1} \mid \mathbb{P}_{2,1 ; \check{\alpha}}^{\lambda, \mu} \cong p_{2}^{*} \mathscr{T}_{2}^{\lambda+\check{\alpha}, \mu+\check{\alpha}} \otimes \mathcal{O}\left(\mathrm{Gr}_{T, X^{2}}^{\lambda, \mu+\check{\alpha}}\right)^{\log M},
$$

where $M$ can be determined in two ways. Over $X^{2} \backslash \Delta$, we have $\mathbb{P}_{2,1 ; \check{\alpha}}^{\lambda, \mu} \cong \mathbb{P}_{1 ; \check{\alpha}}^{\lambda} \times$ $\mathrm{Gr}_{T, X}^{\mu+\check{\alpha}}$ by Lemma II.5.4, so factorizability of $\mathscr{G}_{2}$ and (II.20) give $M=m(\lambda, \check{\alpha})$. On the other hand, clearly $\left.\mathbb{P}_{2,1 ; \check{\alpha}}^{\lambda, \mu}\right|_{\Delta}=\mathbb{P}_{1 ; \check{\alpha}}^{\lambda+\mu+\check{\alpha}}$, so (II.20) gives $M=m(\lambda+\mu+\check{\alpha}, \check{\alpha})$. Since $\mu+\check{\alpha}$ can be any coweight, we conclude that $m(\lambda, \check{\alpha})=m(\check{\alpha})$ is independent of $\lambda$, as desired.

We now compute $m(\check{\alpha})=Q(\check{\alpha})^{-1}$. Let $m: \mathbb{P}_{1 ; \check{\alpha}}^{0} \times \operatorname{Gr}_{T, X}^{\check{\alpha}} \rightarrow \mathbb{P}_{2,1 ; \check{\alpha}}^{0,0}$ be the extension of the factorization map given in Lemma II.5.4 having a zero of order 2 along the divisor $\widetilde{\Delta}$, the fiber over $\Delta \subset X^{2}$. We give two computations of $m^{*}\left(\mathscr{G}_{2} \mid \mathbb{P}_{2,1 ; \check{\alpha}}^{0,0}\right)$ : first, by (II.21), we have

$$
\begin{aligned}
m^{*} \mathscr{G}_{2} & =m^{*}\left(p_{2}^{*} \mathscr{T}_{2}^{\check{\alpha}, \check{\alpha}} \otimes \mathcal{O}\left(\operatorname{Gr}_{T, X^{2}}^{0, \check{\alpha}}\right)^{\log m(\check{\alpha})}\right) \\
& =p_{2}^{*}\left(m^{*} \mathscr{T}_{2}^{\check{\alpha}, \check{\alpha}}\right) \otimes \mathcal{O}\left(\operatorname{Gr}_{T, X^{2}}^{0, \check{\alpha}}\right)^{\log m(\check{\alpha})} \otimes \mathcal{O}(\widetilde{\Delta})^{\log m(\check{\alpha})^{2}} .
\end{aligned}
$$

On the other hand, since $m$ extends the factorization map, we have

$$
m^{*} \mathscr{G}_{2}=\mathscr{G}_{1} \mid \mathbb{P}_{1 ; \check{\alpha}}^{0} \otimes \mathscr{T}_{1}^{\check{\alpha}}
$$

since along the zero section $\operatorname{Gr}_{T, X^{2}}^{0, \check{\alpha}}$, factorization has an order of $\kappa(0, \check{\alpha})=1$. Inserting (II.20), we continue:

$$
m^{*} \mathscr{G}_{2}=\left(p_{1}^{*} \mathscr{T}_{1}^{\check{\alpha}} \otimes \mathcal{O}\left(\mathrm{Gr}_{T, X}^{0}\right)^{\log m(\check{\alpha})}\right) \otimes \mathscr{T}_{1}^{\check{\alpha}} \cong p_{2}^{*}\left(\mathscr{T}_{1}^{\check{\alpha}} \otimes \mathscr{T}_{1}^{\check{\alpha}}\right) \otimes \mathcal{O}\left(\mathrm{Gr}_{T, X^{2}}^{0, \check{\alpha}}\right)^{\log m(\check{\alpha})} .
$$

We have $m^{*} \mathscr{T}_{2}^{\check{\alpha}, \check{\alpha}} \cong \mathscr{T}_{1}^{\check{\alpha}} \otimes \mathscr{T}_{1}^{\check{\alpha}} \otimes \mathcal{O}(\widetilde{\Delta})^{\log \kappa(\check{\alpha}, \check{\alpha})}$ by definition of $\kappa(\check{\alpha}, \check{\alpha})$, and therefore comparing monodromies about the zero and diagonal sections in the above two expressions, we find that $m(\check{\alpha})^{2}=\kappa(\alpha, \alpha)^{-1}=Q(\alpha)^{-2}$. Since the square is induced via a pullback from a 2 -fold cover branched at $\Delta$ on both sides, we find $m(\check{\alpha})=$ $Q(\check{\alpha})^{-1}$, as desired.

Proof of Lemma II.7.6. For 2-morphisms: any factorizable map $\operatorname{Gr}_{G, X^{n}} \rightarrow A$ must be constant on connected components since $A$ is discrete. This is the same as saying that it descends to $\pi_{1}(G)_{n}$.

Now we consider 1-morphisms. Since each $\operatorname{Gr}_{B}^{\lambda}$ is simply-connected, so are their closures, and therefore any $A$-torsor is trivial on each $\overline{\mathrm{Gr}}_{B}^{\lambda}$. Since $\mathrm{Gr}_{G}$ is the union of these nested closed subspaces, any $A$-torsor on $\mathrm{Gr}_{G}$ is trivial (i.e., $\mathrm{Gr}_{G}$ is simplyconnected). Since automorphsims of sf gerbes on $\mathrm{Gr}_{G, X^{n}}$ are sf $A$-torsors, they are all pullbacks along $p: \operatorname{Gr}_{G, X^{n}} \rightarrow \pi_{1}(G)_{X^{n}}$, and thus trivializable. Since 2automorphisms of sf $A$-torsors are factorizable maps to $A$, the previous paragraph shows that in fact such $A$-torsors are uniquely trivializable (as sf torsors).

Now suppose for an sf-gerbe $\mathscr{G}_{n}$ that $\mathscr{T}_{n}=F\left(\mathscr{G}_{n}\right)$ is trivial. Then in particular, it has trivial quadratic form, so by Lemma II.7.7, $\mathscr{G}_{n}$ must be trivial on the fibers of $\mathscr{G}_{n} \rightarrow X^{n}$; therefore, $\mathscr{G}_{n}$ is of the form $p^{*} \mathscr{P}_{n}$, where $\mathscr{P}_{n}$ is factorizable on $\pi_{1}(G)_{X^{n}}$. That is, we have $F\left(\mathscr{T}_{n}\right)=q^{*} \mathscr{P}_{n}$, so $q^{*} \mathscr{P}_{n}$ is trivial on $\mathrm{Gr}_{T, X^{n}}$, and thus $\mathscr{P}_{n}$ is on $X^{n}$. Therefore $\mathscr{G}_{n}$ is trivial on $\mathrm{Gr}_{G, X^{n}}$, and by the previous paragraph, that trivialization is unique (up to unique 2-isomorphism).

Now we may begin the process of lifting $W$-invariant forms to sf gerbes. The following lemma is a useful technical tool for the general argument, though there 
are specific cases of interest (for example, when $A=k^{*}$ with $k$ an algebraically closed field) in which it is unnecessary.

Lemma II.7.8. Suppose that $A \subset B$ are two abelian groups and that $\mathscr{G}_{n}$ is an sf $B$-gerbe such that $F\left(\mathscr{G}_{n}\right)$ is an sf $A$-gerbe on $\operatorname{Gr}_{T, X^{n}}$. Then $\mathscr{G}_{n}$ is defined over $A$.

Proof. It suffices to show that $\mathscr{G}_{n}$ is trivial when induced to $C=B / A$. This is true by hypothesis of $F\left(\mathscr{G}_{n}\right)$, and by injectivity of the map $\mathbf{H}_{\mathrm{sf}}^{2}(G, X, C) \rightarrow \mathbf{H}_{\mathrm{sf}}^{2}(T, X, C)$ (i.e., Lemma II.7.6), the lemma follows.

Proposition II.7.9. For every $Q \in Q\left(\Lambda_{T}, A\right)_{\mathbb{Z}}^{W}$, there exists an sf $A$-gerbe $\mathscr{G}_{n}$ on $\mathrm{Gr}_{G, X^{n}}$ whose associated quadratic form is $Q$.

Proof. Using Lemma II.7.2, we produce an extension $A \subset B$ and elements $a_{i} \in B$ such that $Q$ can be factored as

$$
Q=R \cdot \prod_{i} a_{i}^{Q_{i}}
$$

with the $Q_{i}$ being Killing forms coming from adjoint representations $\operatorname{adj}_{i}$ of $G$ and $R$ descending to $\Lambda_{T} / \Lambda_{T, r}^{\mathbb{Q}}$. By Proposition II.7.5, $R$ comes from some sf gerbe $\mathscr{R}_{n}$ on $\operatorname{Gr}_{G, X^{n}}$, while by Proposition II.6.8, the $Q_{i}$ come from the determinant gerbes $\operatorname{det}\left(\operatorname{adj}_{i}\right)^{\log a_{i}}$. Nominally, the latter are $B$-gerbes, but since their quadratic forms are $A$-valued and their multiplicative parts are trivial, by Proposition II.3.6 the corresponding sf gerbes on $\mathrm{Gr}_{T, X^{n}}$ are defined over $A$, so by Lemma II.7.8, they themselves are actually defined over $A$. Then the $A$-gerbe

$$
\mathscr{G}_{n}=\mathscr{R}_{n} \otimes \prod_{i} \operatorname{det}\left(\operatorname{adj}_{i}\right)^{\log a_{i}}
$$

has quadratic form $Q$.

Corollary II.7.10. If $\mathscr{G}_{n}$ is an sf gerbe, the $\Lambda_{T}$-multiplicative gerbe part of $F\left(\mathscr{G}_{n}\right)$ descends to $\pi_{1}(G)$.

Proof. Clearly, this is true of $\mathscr{R}$ and of $\operatorname{det}\left(\operatorname{adj}_{i}\right)_{n}^{\log a}$ for any $a \in A$ and $G$-representation $V$, so by the preceding construction, we may cancel out the quadratic form of $\mathscr{G}_{n}$ and assume, without loss of generality, that it is trivial. Then by Lemma II.7.7, $\mathscr{G}_{n}$ is trivial on the fibers of $\mathrm{Gr}_{G, X^{n}} \rightarrow X^{n}$ and thus descends to $\pi_{1}(G)_{n}$, as desired.

It remains to show that the functor $F$ actually produces gerbes whose quadratic forms are definable as $W$-invariant forms over $\mathbb{Z}$. This involves technical tools not to be discussed until the next part.

Proposition II.7.11. If $\mathscr{G}_{n}$ is an sf-gerbe, then $Q\left(\mathscr{G}_{n}\right) \in Q\left(\Lambda_{T}, A\right)_{\mathbb{Z}}^{W}$.

Proof. We show in Theorem III.2.10 that $\mathscr{G}_{n}$ possesses a structure of $G(\widehat{\mathcal{O}})_{n}$-equivariance (see Definition III.1.1). Let $G_{n} \subset G(\widehat{\mathcal{O}})_{n}$ be the factorizable subgroup of points $(\vec{x}, g)$ with $g \in G(\bar{x})$, where we consider $\widehat{X}_{\bar{x}}$ to cover $\bar{x}$. Then $\mathscr{G}_{n}$ is $G_{n^{-}}$ equivariant and, thus, equivariant for $N_{G}(T)_{n}$, where $N_{G}(T)$ is the normalizer of the torus $T$, and $N_{G}(T) / T \cong W$ is the Weyl group. This group acts on $\operatorname{Gr}_{T, X^{n}} \subset$ $\operatorname{Gr}_{G, X^{n}}$ through its quotient $W_{n}$, so that $i^{*} \mathscr{G}_{n}$ is necessarily $W_{n}$-equivariant.

Now we show that $Q\left(\mathscr{G}_{n}\right) \in Q\left(\Lambda_{T}, A\right)_{\mathbb{Z}}^{W}$. Since it is $W$-invariant, it suffices by the construction of Proposition II.7.9 and Lemma II.7.2 to show that, if $Q$ is trivial on $\Lambda_{T, r}$, then we can take $k=1$ in the expression there. First, consider the image 
of the grassmannian $\mathrm{Gr}_{G^{\mathrm{sc}}, X^{n}}$ in $\mathrm{Gr}_{G, X^{n}}$, where $G^{\text {sc }}$ is the simply-connected form of $G$, having weight lattice $\Lambda_{T, r}$; its parts are the irreducible components of the $\operatorname{Gr}_{G, X^{n}}$ whose $G(\widehat{\mathcal{O}})_{n}$-orbits (see Section IV.1) are labelled by $\Lambda_{T, r}^{n}$. Thus, since $Q$ vanishes on $\Lambda_{T, r}$, we have by Lemma II.7.6 that $\mathscr{G}_{n}$ is factorizably trivial on these components.

Now we consider the collection of connected components $C$ of $\mathrm{Gr}_{G, X}$ whose $G(\widehat{\mathcal{O}})_{1}$-orbits $\operatorname{Gr}_{G, X}^{\lambda}$ have $\lambda \in \Lambda_{T, r}^{\mathbb{Q}}$ (isomorphic to $\operatorname{Gr}_{G^{\prime}}$, where $G^{\prime}=[G, G]$ is the derived subgroup of $G$ ). In the $k$-fold convolution product (see Definition III.1.4)

$$
m_{k \cdot 1}: \widetilde{\mathrm{Gr}}_{k \cdot 1} \rightarrow \mathrm{Gr}_{k}
$$

the $k$-fold convolution of a single component $C$ maps to $\mathrm{Gr}_{G^{\mathrm{sc}}, X^{k}}$, as described above, since $k \Lambda_{T . r}^{\mathbb{Q}} \subset \Lambda_{T, r}$. By strong factorizable equivariance (Theorem III.2.10 and Definition III.2.4), we have

$$
m_{k \cdot 1}^{*} \mathscr{G}_{k} \cong \mathscr{G}_{1} \widetilde{\otimes} \ldots \widetilde{\nabla} \mathscr{G}_{1}
$$

on these components; for the twisted product, see Definition III.2.2. Since the former is trivial, so is the latter, which we claim implies that $\mathscr{G}_{1}$ is equivariantly trivial. To show this, consider the definition of the twisted product

$$
p^{*} \mathscr{G}_{1}^{\widetilde{\otimes} k}=\pi^{*} \mathscr{G}_{1}^{\widetilde{\otimes}(k-1)} \otimes_{X^{k}} \mathscr{G}_{1},
$$

where $p$ is the $G(\widehat{\mathcal{O}})_{1}$-torsor defined in the proof of Proposition III.1.8 and we write $\pi: \widetilde{G}(\widehat{\mathcal{K}})_{k \cdot 1} \rightarrow \widetilde{\mathrm{Gr}}_{(k-1) \cdot 1}$. Since $\mathscr{G}_{1}^{\widetilde{\otimes} k}$ is trivial, in particular, the second factor on the right is $G(\widehat{\mathcal{O}})_{1}$-equivariantly trivial, as desired.

Finally, since $\mathscr{G}_{1}$ is factorizably trivial on the components $C$, we have that $Q$ is trivial on $\Lambda_{T, r}^{\mathbb{Q}}$, and since it is $G(\widehat{\mathcal{O}})_{1}$-equivariantly trivial, we have by the construction of Theorem III.2.10 that $\kappa\left(\Lambda_{T, r}^{\mathbb{Q}}, \Lambda_{T}\right)=1$; i.e., that $k=1$ in Lemma II.7.2.

This completes the proof of Theorem II.7.3

\section{Part III. Equivariance of symmetric factorizable gerbes}

In this part, we continue to analyze the consequences of the factorizability of a gerbe on the grassmannian. Unlike in the previous part, the principal motivation will be the study of equivariance with respect to a certain group action, which we will see (like our previous theorems) is constructed and constrained simply by the topological data of factorizablity.

\section{III.1. INFINITESIMAL ACTIONS ON THE GRASSMANNIAN}

In this section we finally introduce the spaces of infinitesimal loops that the affine grassmannian is usually defined against.

The formal arc and loop groups. We use the following notation: for any affine scheme $S$ and a point $\vec{x} \in X^{n}(S)$, the graph $\bar{x} \rightarrow X_{S}$ is a closed affine subscheme of $X_{S}$ and thus its $n$th infinitesimal neighborhoods are also affine, with the same underlying topological space $|\bar{x}|$. Letting $\mathcal{O}_{\bar{x}, n}=\mathcal{O}_{\bar{x}_{n}}$ be their rings of global sections on $|\bar{x}|$, we take

$$
\widehat{\mathcal{O}}_{X, \bar{x}}=\lim _{\longleftarrow} \mathcal{O}_{\bar{x}, n}
$$


(which is also the ring of global sections on the formal completion of $X_{S}$ at $\bar{x}$ ) and define

$$
\widehat{X}_{\bar{x}}=\operatorname{Spec} \widehat{\mathcal{O}}_{X, \bar{x}},
$$

which we call the (schemey) formal neighborhood of $\bar{x}$. By definition, $\widehat{\mathcal{O}}_{X, \bar{x}}$ has $\mathcal{O}_{\bar{x}}$ as a quotient, so $\widehat{X}_{\bar{x}}$ has $\bar{x}$ as a closed subscheme, and we define $\widehat{X}_{(\bar{x})}$ to be the complement, the punctured formal neighborhood. We reiterate that neither of these coincide with the like-named concepts in formal schemes.

Definition III.1.1. We define two functors on affine schemes $S$ :

$$
\begin{aligned}
& G(\widehat{\mathcal{O}})_{X^{n}}(S)=\left\{(\vec{x}, g) \mid g \in G\left(\widehat{X}_{x}\right)\right\}, \\
& G(\widehat{\mathcal{K}})_{X^{n}}(S)=\left\{(\vec{x}, g) \mid g \in G\left(\widehat{X}_{(x)}\right)\right\} .
\end{aligned}
$$

We will often write simply $G(\widehat{\mathcal{O}})_{n}$ and $G(\widehat{\mathcal{K}})_{n}$. These are the formal arc group and formal loop group of $G$.

Both of these are, like the $\operatorname{Gr}_{G, X^{n}}$, representable by ind-schemes (in fact, $G(\widehat{\mathcal{O}})_{n}$ is actually a scheme, albeit of infinite type). The following observation is an immediate consequence of the definitions:

Lemma III.1.2. Both $G(\widehat{\mathcal{O}})_{n}$ and $G(\widehat{\mathcal{K}})_{n}$ are sf group ind-schemes.

They are closely related to the grassmannians. The following lemma is a mild generalization of a well-known basic fact, so we only sketch the proof.

Lemma III.1.3. We have a transitive action of sf schemes over $X^{n}$ of $G(\widehat{\mathcal{K}})_{n}$ on $\mathrm{Gr}_{G, X^{n}}$, in which the trivial section (id, $\left.\mathcal{T}^{0}, \mathrm{id}\right)$ has stabilizer $G(\widehat{\mathcal{O}})_{n}$, so we have the quotient as sheaves in the étale topology on affine schemes:

$$
\operatorname{Gr}_{G, X} \cong G(\widehat{\mathcal{K}})_{n} / G(\widehat{\mathcal{O}})_{n} .
$$

Proof. By the Beauville-Laszlo theorem BL95, any point $p=(\vec{x}, \mathcal{T}, \phi) \in \operatorname{Gr}_{n}(S)$ can be equivalently specified by its infinitesimal data:

$$
(\vec{x}, \widehat{\mathcal{T}}, \widehat{\phi}) \quad \widehat{\mathcal{T}}=\mathcal{T}\left|\widehat{X}_{\bar{x}}, \quad \widehat{\phi}=\phi\right| \widehat{X}_{(\bar{x})} .
$$

Then $(\vec{x}, g) \in G(\widehat{\mathcal{K}})_{n}$ acts on $p$ by composing $\widehat{\phi}$ with $g$ on the right, considering $g$ as an automorphism of the trivial torsor on $\widehat{X}_{(\bar{x})}$. The action is transitive because $\widehat{\phi}$ is identified with a group element when $\mathcal{T}$ is trivial, which is locally the case. The stabilizer of the trivial section is $G(\widehat{\mathcal{O}})_{n}$ because up to isomorphism of $\widehat{\mathcal{T}}$, any stabilizing $g$ must be the identity, and thus (not up to isomorphism) must extend across $\bar{x}$, so is in $G(\widehat{\mathcal{O}})_{n}$.

When we consider $\mathrm{Gr}_{n}$ as a quotient of $G(\widehat{\mathcal{K}})_{n}$, we will denote the projection map by $q_{n}$.

Outer convolution diagrams. The most important construction related to the arc groups is that of the following "convolution diagrams". Since it is possible, we give a very general definition, but one should pay particular attention to the case $m=2$. These spaces were described in this case in [MV07, §5]. We use the following notation for partitions: if $p$ is a partition of $n$ into $m$ parts (more properly, a partition of $[1, \ldots, n]$ into $m$ subsets), we will denote the $k$ th part by $p_{k}$, a set, and its cardinality by $\# p_{k}$. 
Definition III.1.4. For any partition $p$ of $n$ into $m$ parts, we have the convolution diagram over $X^{n}$, where $\vec{x}_{k}$ is the collection of coordinates of $\vec{x}$ indexed by the elements of $p_{k}$ :

$$
\widetilde{\operatorname{Gr}}_{G, X^{p}}(S)=\left\{\left(\begin{array}{c}
\vec{x}, \mathcal{T}_{1}, \ldots, \mathcal{T}_{m}, \\
\phi_{1}, \ldots, \phi_{m}
\end{array}\right) \mid \begin{array}{l}
\phi_{1}: \mathcal{T}^{0} \rightarrow \mathcal{T}_{1} \text { is a trivialization on } X \backslash \bar{x}_{1} \\
\phi_{k}: \mathcal{T}_{k-1} \rightarrow \mathcal{T}_{k} \text { is an isomorphism on } X \backslash \bar{x}_{k}
\end{array}\right\} .
$$

As usual, we will denote it $\widetilde{\mathrm{Gr}}_{p}$. We take note of two common special cases: if $p$ is the concatenation of two intervals of sizes $n_{1}$ and $n_{2}$, we will write $p=\left(n_{1}, n_{2}\right)$ and refer to $\widetilde{\mathrm{Gr}}_{n_{1}, n_{2}}$. If $p$ partitions $m n$ as the concatenation of $m$ intervals of equal size $n$, we will write $p=m \cdot n$.

We will have almost no occasion to use convolutions corresponding to partitions other than $p=\left(n_{1}, n_{2}\right)$ or $p=m \cdot n$, so we will assume tacitly that the parts $p_{k}$ are consecutive intervals in $[1, \ldots, n]$. Furthermore, in order to reduce the burden of notation, we will take $m=2$ in all subsequent statements and proofs, with the argument being formally similar for the general case, so that the most general partition we exhibit is $\left(n_{1}, n_{2}\right)$.

We require the convolution diagrams because of their similarities to and differences from products of grassmannians. For example, when $p=\left(n_{1}, n_{2}\right), \widetilde{\mathrm{Gr}}_{n_{1}, n_{2}}$ has the maps

$$
m_{n_{1}, n_{2}}: \widetilde{\mathrm{Gr}}_{n_{1}, n_{2}} \rightarrow \mathrm{Gr}_{n}, \quad \quad \operatorname{pr}_{n_{1}, n_{2}}: \widetilde{\mathrm{Gr}}_{n_{1}, n_{2}} \rightarrow \widetilde{\mathrm{Gr}}_{n_{1}}
$$

The definitions are clear: $m_{n_{1}, n_{2}}$ is defined combinatorially as

$$
m_{n_{1}, n_{2}}\left(\vec{x}, \mathcal{T}_{1}, \mathcal{T}_{2}, \phi_{1}, \phi_{2}\right)=\left(\vec{x}, \mathcal{T}_{2},\left.\left.\phi_{2}\right|_{X_{S} \backslash \bar{x}} \circ \phi_{1}\right|_{X_{S} \backslash \bar{x}}\right),
$$

and $\operatorname{pr}_{n_{1}, n_{2}}$ is defined by forgetting both $\mathcal{T}_{2}$ and $\phi_{2}$. More generally, we have maps $m_{p}: \widetilde{\mathrm{Gr}}_{p} \rightarrow \mathrm{Gr}_{n}$ and $\mathrm{pr}_{p}: \widetilde{\mathrm{Gr}}_{p} \rightarrow \widetilde{\mathrm{Gr}}_{p^{\prime}}$, where $p^{\prime}$ is obtained by removing the last part $p_{m}$ from $p$. We proceed with establishing the good properties of these maps.

Proposition III.1.5. On the open set $X_{p}^{n}$ of Proposition II.1.3, we have an isomorphism of $\widetilde{\mathrm{Gr}}_{p}$ with the product of the $\mathrm{Gr}_{\# p_{k}}$. In addition, the map $m_{p}$ is ind-proper and its restriction to $X_{p}^{n}$ is the factorization isomorphism of Proposition II.1.3)(2).

Proof. We take $p=\left(n_{1}, n_{2}\right)$. We will see shortly that $\operatorname{pr}_{n_{1}, n_{2}}$ realizes $\widetilde{G r}_{n_{1}, n_{2}}$ as a $\mathrm{Gr}_{n_{2}}$-bundle over $\mathrm{Gr}_{n_{1}}$; since they are both ind-proper, so is the convolution diagram, and thus so is any map out of it. Independently of this result, however, we see that we have a trivialization $\psi=\phi_{2} \circ \phi_{1}$ (suitably restricted, as above) of $\mathcal{T}_{2}$, on the open set $X_{S} \backslash \bar{x}$. Since $X_{n_{1}, n_{2}}^{n}$ has as points those $\vec{x}$ with $\bar{x}_{1} \cap \bar{x}_{2}=\varnothing$, we may perform a gluing with the trivial torsor,

$$
\mathcal{T}_{2}^{\prime}=\left.\left.\mathcal{T}_{2}\right|_{X_{S} \backslash \bar{x}_{1}} \sqcup_{\psi} \mathcal{T}^{0}\right|_{X_{S} \backslash \bar{x}_{2}}
$$

and the two triples $\left(\vec{x}_{1}, \mathcal{T}_{1}, \phi_{1}\right)$ and $\left(\vec{x}_{2}, \mathcal{T}_{2}^{\prime}, \psi\right)$ are points of $\mathrm{Gr}_{n_{i}}$ for $i=1,2$, as desired.

Conversely, given two such triples (with the same notation) we construct a point of $\widetilde{G r}_{n_{1}, n_{2}}$ by reverse gluing: on their common domain of definition $X_{S} \backslash\left(\bar{x}_{1} \cup \bar{x}_{2}\right)=$ $X_{S} \backslash \bar{x}, \phi_{1}$ and $\psi$ are both trivializations and, therefore, $\phi_{2}=\phi_{1} \psi^{-1}: \mathcal{T}_{2}^{\prime} \rightarrow \mathcal{T}_{1}$ is an isomorphism; we define

$$
\mathcal{T}_{2}=\left.\left.\mathcal{T}_{2}^{\prime}\right|_{X_{S} \backslash \bar{x}_{1}} \sqcup_{\phi_{2}} \mathcal{T}_{1}\right|_{X_{S} \backslash \bar{x}_{2}}
$$


and then $\left(\vec{x}, \mathcal{T}_{1}, \mathcal{T}_{2}, \phi_{1}, \phi_{2}\right) \in \widetilde{\mathrm{Gr}}_{n_{1}, n_{2}}(S)$. It is clear that these two constructions invert each other.

Finally, that $m_{n_{1}, n_{2}}$ sends this isomorphism to the factorization map is a matter of comparing the above construction to the proof of Proposition II.1.3.

We now turn to the relationship between the convolution diagram and products of the grassmannians. They are not equal; however, as already claimed, the convolution diagram is a twisted product. To properly express this, we introduce another ind-representable functor:

Definition III.1.6. As before, we write $\vec{x}=\left(\vec{x}_{1}, \vec{x}_{2}\right)$, with $\vec{x}_{i}$ having $n_{i}$ coordinates. We define the following functor on affine schemes $S$ :

$$
\widetilde{G}(\widehat{\mathcal{K}})_{X^{n_{1}, n_{2}}}(S)=\left\{\begin{array}{l|l}
(\vec{x}, \mathcal{T}, \phi, \psi) & \begin{array}{l}
\left(\vec{x}_{1}, \mathcal{T}, \phi\right) \in \widetilde{\mathrm{Gr}}_{n_{1}} \\
\psi: \mathcal{T}^{0} \rightarrow \mathcal{T} \text { is a trivialization on } \widehat{X}_{\bar{x}_{2}}
\end{array}
\end{array}\right\} .
$$

As usual, we will write just $\widetilde{G}(\widehat{\mathcal{K}})_{n_{1}, n_{2}}$.

There is an obvious map $\widetilde{G}(\widehat{\mathcal{K}})_{n_{1}, n_{2}} \rightarrow \mathrm{Gr}_{n_{1}}$ given by forgetting $\vec{x}_{2}$ and $\psi$, and there is also an obvious action of the relative group $X^{n_{1}} \times G(\widehat{\mathcal{O}})_{n_{2}}$ on $\widetilde{G}(\widehat{\mathcal{K}})_{n_{1}, n_{2}}$, with a point $\left(\vec{x}_{1}, \vec{x}_{2}, g\right)$ replacing $\psi$ by $\psi g^{-1}$. This action in fact makes it a torsor for this group over $\mathrm{Gr}_{n_{1}} \times X^{n_{2}}$, with the following self-evident trivializations:

Lemma III.1.7. The $G(\widehat{\mathcal{O}})_{n_{2}}$-torsor $\widetilde{G}(\widehat{\mathcal{K}})_{n_{1}, n_{2}}$ is trivialized over $\operatorname{Gr}_{n_{1}} \times X^{n_{2}}$ as follows:

(1) On $X_{n_{1}, n_{2}}^{n}$, there is a natural projection over $\widetilde{\mathrm{Gr}}_{n_{1}}$ to $G(\widehat{\mathcal{O}})_{n_{2}}$,

$$
(\vec{x}, \mathcal{T}, \phi, \psi) \mapsto u=\psi^{-1} \circ \phi
$$

(for partitions with $m>1$, this trivialization works on the slightly larger open set of $\vec{x}$ 's such that $\vec{x}_{m}$ is disjoint from all $\vec{x}_{k}$, with $k<m$ ).

(2) For any $\vec{x}$, there is an unnatural projection associated with each choice of trivialization $\psi^{\prime}$ of $\mathcal{T}$ on $\widehat{X}_{\bar{x}_{2}}$ :

$$
(\vec{x}, \mathcal{T}, \phi, \psi) \mapsto v=\psi^{-1} \circ \psi^{\prime} .
$$

We note that in the more general situation where $p$ is a larger partition, $\widetilde{G}(\widehat{\mathcal{K}})_{p}$ is a torsor over $\widetilde{G r}_{p^{\prime}} \times X^{\# p_{m}}$, where $p^{\prime}$ is the partition obtained by deleting the last part $p_{m}$ of $p$. Now we can say that the convolution diagram is the twisting of an ordinary product of grassmannians by this torsor.

Proposition III.1.8. $\widetilde{G r}_{n_{1}, n_{2}}$ is the bundle associated with $\widetilde{G}(\widehat{\mathcal{K}})_{n_{1}, n_{2}}$ having fiber $\mathrm{Gr}_{n_{2}}$. Explicitly, this means that

$$
\widetilde{\operatorname{Gr}}_{n_{1}, n_{2}}=\left(\widetilde{G}(\widehat{\mathcal{K}})_{n_{1}, n_{2}} \times_{X^{n_{2}}} \operatorname{Gr}_{n_{2}}\right) / G(\widehat{\mathcal{O}})_{n_{2}},
$$

where the latter group acts anti-diagonally on the product. In particular, the trivialization of Lemma III.1.] (1) gives an isomorphism over $X_{n_{1}, n_{2}}^{n}$ :

$$
\widetilde{\mathrm{Gr}}_{n_{1}, n_{2}} \cong \mathrm{Gr}_{n_{1}} \times \mathrm{Gr}_{n_{2}}
$$

and this isomorphism agrees with the one given in Proposition III.1.5. For more general partitions $p$, if $p^{\prime}$ is the partition obtained by removing the last part $p_{m}$ of $p$, then we have that $\widetilde{\mathrm{Gr}}_{p}$ is a $\mathrm{Gr}_{\# p_{m}}$-bundle over $\widetilde{\mathrm{Gr}}_{p^{\prime}}$. 
Proof. We begin by defining a map $\pi: \widetilde{G}(\widehat{\mathcal{K}})_{n_{1}, n_{2}} \times_{X^{n_{2}}} \mathrm{Gr}_{n_{2}} \rightarrow \widetilde{G r}_{n_{1}, n_{2}}$, which on $S$-points looks like

$$
\pi:\left(\left(\vec{x}_{1}, \vec{x}_{2}, \mathcal{T}_{1}, \phi_{1}, \psi\right),\left(\vec{x}_{2}, \mathcal{T}_{2}, \phi_{2}\right)\right) \mapsto\left(\vec{x}, \mathcal{T}_{1}, \mathcal{T}_{2}^{\prime}, \phi_{1}, \phi_{2}^{\prime}\right),
$$

where $\mathcal{T}_{2}^{\prime}$ is the gluing of $\mathcal{T}_{1} \mid\left(X \backslash \bar{x}_{2}\right)$ with $\mathcal{T}_{2} \mid \widehat{X}_{\bar{x}_{2}}$ along the isomorphism $\phi_{2} \circ \psi^{-1}$, and $\phi_{2}^{\prime}$ is the natural isomorphism with $\mathcal{T}_{1}$ away from $\bar{x}_{2}$.

Evidently its fibers are invariant under the action of $G(\widehat{\mathcal{O}})_{n_{2}}$, any element $g$ of which sends $\psi$ to $\psi g$ and $\phi_{2}$ to $\phi_{2} g$, so the $g$ cancels in the gluing. Conversely, given any tuple in the target, we can locally on $S$ choose a trivialization $\psi$ of $\mathcal{T}_{1}$ around $\bar{x}_{2}$ and define $\mathcal{T}_{2}$ by gluing $\mathcal{T}_{2}^{\prime} \mid \widehat{X}_{\bar{x}_{2}}$ to the trivial torsor via $\phi_{2}^{\prime} \circ \psi$. This shows that $\pi$ is locally surjective and that its fibers are $G(\widehat{\mathcal{O}})_{n_{2}}$-torsors. The final claim about the isomorphisms follows by chasing the constructions.

Inner convolution diagrams. An alternative construction of the convolution diagram that we will use is one which perhaps looks more like the convolution diagram usually defined on $\mathrm{Gr}_{G}$.

Definition III.1.9. The inner convolution diagrams are the following ind-schemes over $X^{n}$, where $m \geq 1$ :

$$
\operatorname{Conv}_{n}^{m}=G(\widehat{\mathcal{K}})_{n} \times^{G(\widehat{\mathcal{O}})_{n}} \ldots \times^{G(\widehat{\mathcal{O}})_{n}} G(\widehat{\mathcal{K}})_{n} \times^{G(\widehat{\mathcal{O}})_{n}} \mathrm{Gr}_{n},
$$

where there are $m$ terms in all.

Proposition III.1.10. Let $p$ be the partition of $m n$ into $m$ copies of $n$ (we write $p=m \cdot n)$. Then we have:

(1) $\mathrm{Conv}_{n}^{m}$ is isomorphic to the restriction of $\widetilde{\mathrm{Gr}}_{p}$ to the diagonal $\Delta \subset X^{m n}$ where coordinates $x_{i}=x_{i+m}=\ldots$ for $i=1, \ldots, n$.

(2) For $m \geq 2$, the restriction of $\widetilde{G}(\widehat{\mathcal{K}})_{p}$ to $\Delta$ is the twisted product

$$
\operatorname{Conv} G(\widehat{\mathcal{K}})_{n}^{m}=G(\widehat{\mathcal{K}})_{n} \times^{G(\widehat{\mathcal{O}})_{n}} \times \cdots \times \times^{G(\widehat{\mathcal{O}})_{n}} G(\widehat{\mathcal{K}})_{n}
$$

with $m-1$ terms in all.

Proof. By Lemma III.1.3, we have $\mathrm{Gr}_{n}=G(\widehat{\mathcal{K}})_{n} / G(\widehat{\mathcal{O}})_{n}$, so that Conv $G(\widehat{\mathcal{K}})_{n}^{m}$ is clearly a $G(\widehat{\mathcal{O}})_{n}$-torsor over $\operatorname{Conv}_{n}^{m}$, and $\operatorname{Conv}_{n}^{m}$ is clearly the twisting of Conv $G(\widehat{\mathcal{K}})_{n}^{m}$ with $\mathrm{Gr}_{n}$. By Proposition III.1.8, therefore, it suffices to show only (2).

When $m=2$, this is the claim that $\left.\widetilde{G}(\widehat{\mathcal{K}})_{2 \cdot n}\right|_{\Delta}=G(\widehat{\mathcal{K}})_{n}$. Indeed, the left-hand side is the moduli space of data $\left(\vec{x}, \mathcal{T}_{1}, \phi_{1}, \psi\right)$, where $\vec{x} \in X^{n}(S), \mathcal{T}_{1}$ is a $G$-torsor on $X_{S}, \phi_{1}$ is its trivialization on $X_{S} \backslash \bar{x}$, and $\psi$ is its trivialization on $\widehat{X}_{\bar{x}}$. By the Beauville-Laszlo theorem, the latter data is equivalent to giving $\psi^{-1} \phi_{1} \in G\left(\widehat{X}_{(\bar{x})}\right)$, as claimed.

In general, we claim that

$$
\left.\widetilde{G}(\widehat{\mathcal{K}})_{m \cdot n}\right|_{\Delta}=\left.\left(\widetilde{G}(\widehat{\mathcal{K}})_{(m-1) \cdot n} \times{ }^{G(\widehat{\mathcal{O}})_{n}} G(\widehat{\mathcal{K}})_{n}\right)\right|_{\Delta} .
$$

Since this is also true of Conv $G(\widehat{\mathcal{K}})_{n}^{m}$, the proposition would follow by induction. (In fact, we could remove the $\Delta$ 's, but will not need to.) The proof is quite similar to that of Proposition III.1.8 for clarity we exemplify it with the case $m=3$. We construct a map from the right-hand side to the left-hand side, starting with a pair of tuples

$$
(\vec{x}, \mathcal{T}, \phi, \psi) \in \widetilde{G}(\widehat{\mathcal{K}})_{2 \cdot n}(S), \quad(\vec{x}, g) \in G(\widehat{\mathcal{K}})_{n}(S)
$$


(having the same $\vec{x}$, since we restrict to $\Delta$ ). Thus, $\phi$ trivializes $\mathcal{T}$ over $X_{S} \backslash \bar{x}$ and $\psi$ trivializes it on $\widehat{X}_{\bar{x}}$, while $g \in G\left(\widehat{X}_{(\bar{x})}\right)$. We take $\mathcal{T}_{1}=\mathcal{T}$ and define $\mathcal{T}_{2}$ by gluing, using the Beauville-Laszlo theorem:

$$
\mathcal{T}_{2}=\left.\left.\mathcal{T}_{1}\right|_{X_{S} \backslash \bar{x}} \sqcup_{g \psi^{-1}} \mathcal{T}^{0}\right|_{\widehat{X}_{\bar{x}}} .
$$

Then $\left(\vec{x}, \mathcal{T}_{1}, \mathcal{T}_{2}, \phi\right.$, can., can. $) \in \widetilde{G}(\widehat{\mathcal{K}})_{3 \cdot n}$, where the maps "can." denote, respectively, the canonical isomorphism of $\mathcal{T}_{2}$ with $\mathcal{T}_{1}$ on $X_{S} \backslash \bar{x}$ and the canonical trivialization of $\mathcal{T}_{2} \mid \widehat{X}_{\bar{x}}$.

Conversely, given a tuple

$$
\left(\vec{x}, \mathcal{T}_{1}, \mathcal{T}_{2}, \phi_{1}, \phi_{2}, \psi\right) \in \widetilde{G}(\widehat{\mathcal{K}})_{3 \cdot n}(S),
$$

locally on $S$ we may choose a trivialization $\psi^{\prime}$ of $\mathcal{T}_{1}$ on $\widehat{X}_{\bar{x}}$ and simply define $g=\psi^{-1} \circ \phi_{2} \circ \psi^{\prime}$. Then $\left(\vec{x}, \mathcal{T}_{1}, \phi_{1}, \psi^{\prime}\right) \in \widetilde{G}(\widehat{\mathcal{K}})_{2 \cdot n}(S),(\vec{x}, g) \in G(\widehat{\mathcal{K}})_{n}$, and $\mathcal{T}_{2}$ is by definition the gluing of $\mathcal{T}_{1}$ and the trivial torsor as above. This identifies the fibers of the previous map with $G(\widehat{\mathcal{O}})_{n}$, as desired.

Using these special convolution diagrams, we can express the maps $m_{p}$ of (III.1) in terms of the actual multiplication maps on $G(\widehat{\mathcal{K}})_{n}$. The proof follows by comparing (III.1) with the above construction.

Corollary III.1.11. After Proposition III.1.10|(1), the map $\mathrm{Conv}_{n}^{m} \rightarrow \mathrm{Gr}_{n}$, given by multiplying all the $G(\widehat{\mathcal{K}})_{n}$ coordinates and applying the result to the $\mathrm{Gr}_{n}$ coordinate, is the same as $\left.m_{p}\right|_{\Delta}$, where $p=m \cdot n$ and $\Delta \subset X^{m n}$ as in the proposition.

Although we have exhibited the inner convolution diagrams as special cases of the outer ones, in fact, there is a similar relationship in the other order. In order to connect with Definition III.1.4 in full generality, we consider (for once) arbitrary partitions $p$.

Proposition III.1.12. Let $p$ be any partition of $n$ with $m$ parts. Then $\widetilde{\mathrm{Gr}}_{p}$ is the subspace of $\mathrm{Conv}_{n}^{m}$ whose $k$ th coordinate in $G(\widehat{\mathcal{K}})_{n}$ (using the product representation of Definition III.1.9) parametrizes the pairs $(\vec{x}, g)$ such that $g$ extends to $\widehat{X}_{\bar{x}} \backslash \bar{x}_{k}$, where $\vec{x}=\left(\vec{x}_{1}, \ldots, \vec{x}_{m}\right)$ is partitioned according to $p$. In addition, $\widetilde{G}(\widehat{\mathcal{K}})_{p}$ is the restriction of $\operatorname{Conv} G(\widehat{\mathcal{K}})_{n}^{m}$ to this subspace.

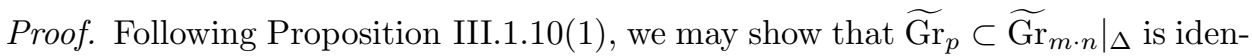
tified with the subspace consisting of points $\left(\vec{x},\left\{\mathcal{T}_{k}\right\},\left\{\phi_{k}\right\}\right)$ such that each isomorphism $\phi_{k}$ extends to $X_{S} \backslash \bar{x}_{k}$. This is the actual definition, however.

Likewise, for $\widetilde{G}(\widehat{\mathcal{K}})_{p}$, we can show that it is identified with the subspace of $\widetilde{G}(\widehat{\mathcal{K}})_{m \cdot n}$ consisting of points $\left(\vec{x},\left\{\mathcal{T}_{k}\right\}_{k<m},\left\{\phi_{k}\right\}_{k<m}, \psi\right)$ for which the $\phi_{k}$ 's extend as above and for which $\psi$ extends to $X_{S} \backslash \bar{x}_{m}$, which is once again the definition.

Corollary III.1.13. Let $p$ be any partition of $n$. There is an action of $G(\widehat{\mathcal{O}})_{n}$ on $\widetilde{\mathrm{Gr}}_{p}$ such that the map $m_{p}$ is $G(\widehat{\mathcal{O}})_{n}$-equivariant.

Proof. $G(\widehat{\mathcal{O}})_{n}$ acts on $\operatorname{Conv}_{n}^{m}$ by multiplying the first coordinate on the left, and by Corollary III.1.11 this makes $\left.m_{p}\right|_{\Delta}$ equivariant when $p=m \cdot n$. For more general $p$, we note that this action preserves $\widetilde{G r}_{p} \subset \operatorname{Conv}_{n}^{m}$, following the description in Proposition III.1.12.

Having these variants of the same definition will play a key role in the theorem which we will prove in the next section. 
Special properties of the torus. Since when $G=T$ is a torus it is commutative, its torsors admit a tensor product and, as previously, this gives a simplification of the factorizable structure of $\mathrm{Gr}_{T, X^{n}}$ and allied spaces.

Lemma III.1.14. For any partition $p$ into $m$ parts, the factorization of the $T(\widehat{\mathcal{O}})_{n_{2}}{ }^{-}$ bundle of Proposition III.1.8,

$$
\widetilde{T}(\widehat{\mathcal{O}})_{p} \times_{X \# p_{m}} \operatorname{Gr}_{T, X \# p_{m}} \stackrel{\pi}{\rightarrow} \widetilde{\mathrm{Gr}}_{p}
$$

descends to a factorization of $\widetilde{\mathrm{Gr}}_{p}$ extending the natural factorzation over $X_{p}^{n}$,

$$
\widetilde{\mathrm{Gr}}_{p} \cong \widetilde{\mathrm{Gr}}_{p^{\prime}} \times \mathrm{Gr}_{T, X \# p_{m}}
$$

Furthermore, the map $m_{p}$ then becomes the multiplication map.

Proof. The action of $T(\widehat{\mathcal{O}})_{\# p_{m}}$ on $\mathrm{Gr}_{\# p_{m}}$ is trivial, since $T$ is commutative, so it acts on the above bundle only through the first factor. Thus, the product decomposition descends to the base, as claimed.

For simplicity, suppose $p=\left(n_{1}, n_{2}\right)$. Composing the constructions of Proposition III.1.8 and Proposition III.1.5, we see that the point $(\vec{x}, \mathcal{T}, \phi) \in \operatorname{Gr}_{T, X^{n_{2}}}$ obtained by applying factorization to $\pi\left(\vec{x}, \mathcal{T}_{1}, \phi_{1}, \psi, \mathcal{T}_{2}, \phi_{2}\right)$ is the torsor $\mathcal{T}$ obtained by gluing $\mathcal{T}_{2} \mid \widehat{X}_{\bar{x}_{2}}$ to the trivial torsor on $X \backslash \bar{x}_{2}$ along the trivialization $\phi_{2} \circ\left(\psi^{-1} \circ \phi_{1}\right)$, and $\phi$ is the natural trivialization on $X \backslash \bar{x}_{2}$. Since $\psi^{-1} \circ \phi_{1} \in T(\widehat{\mathcal{O}})_{n_{2}}$, which acts trivially on $\operatorname{Gr}_{T, X^{n_{2}}}$, we have $(\mathcal{T}, \phi) \cong\left(\mathcal{T}_{2}, \phi_{2}\right)$, so that both definitions of factorization agree.

An alternative way of describing the factorization of the convolution product is as follows, for $\left(\vec{x}, \mathcal{T}_{1}, \mathcal{T}_{2}, \phi_{1}, \phi_{2}\right) \in \widetilde{G r}_{n_{1}, n_{2}}(S)$ and $\left(\vec{x}_{i}, \mathcal{T}_{i}^{\prime}, \phi_{i}^{\prime}\right) \in \mathrm{Gr}_{n_{i}}(i=1,2)$, we have $\left(\mathcal{T}_{1}^{\prime}, \phi_{1}^{\prime}\right)=\left(\mathcal{T}_{1}, \phi_{1}\right)$ and

$$
\begin{gathered}
\left(\mathcal{T}_{2}^{\prime}, \phi_{2}^{\prime}\right)=\left(\mathcal{T}_{1}^{-1} \otimes \mathcal{T}_{2}, \mathrm{id} \otimes \phi_{2}\right), \\
\left(\mathcal{T}_{2}, \phi_{2}\right)=\left(\mathcal{T}_{1}^{\prime} \otimes \mathcal{T}_{2}^{\prime}, \mathrm{id} \otimes \phi_{2}^{\prime}\right) .
\end{gathered}
$$

Since the multiplication map produces $(\mathcal{T}, \phi) \in \mathrm{Gr}_{n_{1}+n_{2}}(S)$ with $\mathcal{T}=\mathcal{T}_{1}^{\prime} \otimes \mathcal{T}_{2}^{\prime}$ and $\phi=\phi_{1}^{\prime} \otimes \phi_{2}^{\prime}$, and $m_{p}$ gives $\mathcal{T}=\mathcal{T}_{2}$ with $\phi=\phi_{2} \circ \phi_{1}$, the two agree with the understanding that the composition of maps of $T$-torsors and their product are the same.

We are inspired to use the notation $\widetilde{\mathrm{Gr}_{p}^{\lambda}} \lambda_{1}, \ldots, \lambda_{n}$ to refer to the twisted product of the individual components of $\mathrm{Gr}_{n}$ indexed by the $\lambda$ 's in this order. We use the same indexing for $\widetilde{G}(\widehat{\mathcal{K}})_{p}$ as for $\widetilde{G r}_{p^{\prime}}$ (that is, the " $G(\widehat{\mathcal{O}})_{\# p_{m}}$ part" does not contribute indices).

This lemma is interesting on account of the nontriviality of the torsor $\widetilde{T}(\widehat{\mathcal{O}})_{p}$ even though $T$ is a torus. In fact, via that space we can give another description of the bilinear form of Definition II.3.1 To do so, we indulge in some generalities; here, the notion of multiplicative torsor is a simplification of that for gerbes.

Lemma III.1.15. Let $A$ be an abelian group. An A-torsor $\mathcal{T}$ on $T$ is identified with a homomorphism $\phi: \Lambda_{T} \rightarrow A$; all such torsors are multiplicative. For any $\lambda \in \Lambda_{T}=\operatorname{Hom}\left(\mathbf{G}_{\mathbf{m}}, T\right)$, we have $\lambda^{*} \mathcal{T}=\mathcal{L}_{\phi(\lambda)}$, where for any $a \in A, \mathcal{L}_{a}$ is, as before Proposition I.5.3, the local system of rank 1 on $\mathbf{G}_{\mathbf{m}}$ with monodromy a. 
Proof. In general, $A$-torsors on a space are identified with characters of the fundamental group by the above prescription; we identify $\Lambda_{T}$ with $\pi_{1}(T, 1)$. The multiplicativity follows from the fact that all the classes in $\pi_{1}(T, 1)$ are homomorphisms $\mathbf{G}_{\mathbf{m}} \rightarrow T$.

We now give a generalization of the construction of Proposition I.5.3 as for multiplicative torsors, the notion of twisted equivariance is similar to that for gerbes. Let $\mathcal{T}$ be a $T$-torsor on a space $Y$, and let $E$ be its total space. Then for any homomorphism $\phi: \Lambda_{T} \rightarrow A$, denoting $\mathcal{L}_{\phi}$ the associated multiplicative $A$-torsor on $T$, we define $\mathcal{T}^{\log \phi}$ to be the gerbe whose sections over $U \subset Y$ are the $\mathcal{L}_{\phi}$-twisted $T$-equivariant $A$-torsors on $\left.E\right|_{U}$. Since $E$ is locally trivial, we see that this is indeed an $A$-gerbe, as before.

Let $D \subset Y$ be a smooth divisor; we will say that a trivialization $\left.\mathcal{T}^{0} \rightarrow \mathcal{T}\right|_{Y \backslash D}$ has order $\lambda$ for $\lambda \in \Lambda_{T}$ if it induces an isomorphism $\mathcal{T} \cong \mathcal{O}(\lambda D)$, in the notation of Proposition II.1.2)(1), The following proposition generalizes Proposition I.5.3 and has, essentially, the same proof (the coweight $\lambda$ enters the computation as the change of trivialization, in place of the parameter $z$ used there).

Proposition III.1.16. Suppose we have a trivialization $u$ of order $\lambda$ as above; then the induced trivialization of $\mathcal{T}^{\log \phi}$ has order $\phi(\lambda)$ in the sense of Lemma I.5.1,

Now we can characterize a familiar gerbe using the trivializations $u$ and $v$ of Lemma III.1.7. We consider the not-quite $T$-torsors $\widetilde{T}(\widehat{\mathcal{O}})_{p}$, where the base space is $\widetilde{\mathrm{Gr}}_{p^{\prime}}$ and $\# p_{m}=1$. These are $T(\widehat{\mathcal{O}})_{1}$-torsors, but we have a natural map $T(\widehat{\mathcal{O}})_{1} \rightarrow$ $T \times X$, where for $g \in T\left(\widehat{X}_{\bar{x}}\right)$ the $S$-point $(x, g)$ goes to $\left(x,\left.g\right|_{S}\right)$, with $S \cong \bar{x} \subset \widehat{X}_{\bar{x}}$ identified with its graph. We will refer to the $T$-torsor on $\widetilde{G r}_{p^{\prime}} \times X$ associated with $\widetilde{T}(\widehat{\mathcal{O}})_{p}$ as $\mathcal{T}_{p}$.

For simplicity, we will consider $p=(n-1,1)$ for some integer $n$. Then for weights $\lambda_{1}, \ldots, \lambda_{n-1} \in \Lambda_{T}$, by Proposition II.1.2 we identify $\operatorname{Gr}_{n-1}^{\lambda_{1}, \ldots, \lambda_{n-1}} \cong X^{n-1}$, and thus $\mathcal{T}_{n-1,1}$ with a $T$-torsor on $X^{n}$. We call $\pi_{n-1,1}: \widetilde{T}(\mathcal{\mathcal { O }})_{n-1,1} \rightarrow X^{n}$ the structure map.

Proposition III.1.17. Let $\kappa: \Lambda_{T} \otimes \Lambda_{T} \rightarrow A$ be a bilinear form, and for any $\mu \in \Lambda_{T}$, let $\kappa_{\mu}(\bullet)=\kappa(\bullet, \mu)$. Then the trivialization $u$ on the complement of the divisors $\Delta_{\text {in }}$ for $i<n$ makes $\mathcal{T}_{n-1,1}^{\log \kappa_{\mu}} \cong \bigotimes_{i} \mathcal{O}\left(\Delta_{i n}\right)^{\log \kappa\left(\lambda_{i}, \mu\right)}$.

Proof. By Proposition II.1.2, the trivialization $u$ of $\mathcal{T}_{n-1,1}$ on $X^{n} \backslash \bigcup \Delta_{\text {in }}$ (from Lemma III.1.7) has order $\lambda_{i}$ about $\Delta_{i n}$. Then by Proposition III.1.16, we find that $\mathcal{T}_{n-1,1}^{\log \kappa_{\mu}}$ has order $\kappa_{\mu}\left(\lambda_{i}\right)$ about $\Delta_{i n}$, as desired.

Returning to the generalities, we note the following fact:

Lemma III.1.18. Let $\pi: E \rightarrow Y$ be the structure map; then $\pi^{*} \mathcal{T}^{\log \phi}$ is trivial.

Proof. Sections of $\pi^{*} \mathcal{T}^{\log \phi}$ are identified with $\mathcal{L}_{\phi}$-twisted equivariant torsors on the $T$-bundle $E \times_{Y} E$ over $E$. Since $E$ is a $T$-torsor, this is the same as $T \times E$; then $\operatorname{pr}_{T}^{*} \mathcal{L}_{\phi}$ itself is such a torsor.

In our particular situation, this implies:

Corollary III.1.19. For any $\phi: \Lambda_{T} \rightarrow A, \pi_{n-1,1}^{*} \mathcal{T}_{n-1,1}^{\log \phi}$ is trivial.

Proof. There is a natural map over $X^{n}, \widetilde{T}(\widehat{\mathcal{O}})_{n-1,1} \rightarrow \mathcal{T}_{n-1,1}$, and so this follows from Lemma III.1.18 by pulling back. 


\section{III.2. FACTORIZABLE GERBES ARE NATURALLY EQUIVARIANT}

The main theorem of this section is that factorizable gerbes automatically enjoy an essentially unique structure relating to the infinitesimal actions on the grassmannian. To describe this structure, we begin with a sequence of definitions.

Definition III.2.1. Let $\mathscr{G}_{n}$ be an sf gerbe. The structure of factorizable equivariance for $G(\widehat{\mathcal{O}})_{n}$ is an equivariance structure on each individual gerbe together with compatibilities of these structures with all the factorization data. Equivalently, it is a structure of equivariance for the action of the sf group scheme $G(\widehat{\mathcal{O}})_{n}$ on $\mathrm{Gr}_{n}$.

We maintain the expository convention of considering only partitions $p=\left(n_{1}, n_{2}\right)$ of a number $n$ or $p=m \cdot n$ of the product $m n$.

Definition III.2.2. Let $\mathscr{G}_{n}$ be a factorizably equivariant sf gerbe. The twisted product on any $\widetilde{\mathrm{Gr}}_{n_{1}, n_{2}}$ is defined by the formula

$$
\mathscr{G}_{n_{1}} \widetilde{\otimes} \mathscr{G}_{n_{2}}=\operatorname{pr}_{n_{1}, n_{2}}^{*} \mathscr{G}_{n_{1}} \otimes \widetilde{\mathscr{G}}_{n_{2}}
$$

where $\operatorname{pr}_{n_{1}, n_{2}}$ is, as after (III.1), the projection $\widetilde{G r}_{n_{1}, n_{2}} \rightarrow \mathrm{Gr}_{n_{1}}$, and the twisted pullback refers to the $G(\mathcal{O})_{n_{2}}$-torsor $\widetilde{G}(\widehat{\mathcal{K}})_{p}$ as in Definition I.3.13 By similar measures, we have a twisted product on $\operatorname{Conv}_{n}^{m}$, and it is easy to see that they agree under the identification of Proposition III.1.12. Using this twisted product, or using the generalization of Proposition III.1.8 to arbitrary partitions, we can define twisted products for any partition, and either route will give equivalent results for the same reason as above.

Lemma III.2.3. With the setup of Definition III.2.2, if $p$ is a partition of $n$, then the twisted product is equivariant for $G(\widehat{\mathcal{O}})_{n}$, relative to the action of this group given in Corollary III.1.13.

Proof. By Proposition III.1.12 it suffices to verify this for twisted products on $\operatorname{Conv}_{n}^{m}$. The action of $G(\hat{\mathcal{O}})_{n}$ on that space is simply on the first factor, whereas $\widetilde{\mathscr{G}}_{n}$ is the descent of a pullback from the last factor, so unless $m=1$ it is trivially $G(\widehat{\mathcal{O}})_{n}$-equivariant; when $m=1$ the whole construction is trivial.

Factorizable equivariance is not enough for our purposes because it has a loose end. To see this, consider the map $m_{n_{1}, n_{2}}: \widetilde{G r}_{n_{1}, n_{2}} \rightarrow \mathrm{Gr}_{n}$, which by Proposition III.1.5, is naturally identified with the factorization isomorphism $\mathrm{Gr}_{n} \cong$ $\mathrm{Gr}_{n_{1}} \times \mathrm{Gr}_{n_{2}}$ on the open set $X_{n_{1}, n_{2}}^{n}$. By Proposition III.1.8 the projection

$$
\operatorname{pr}_{n_{1}, n_{2}}: \widetilde{\mathrm{Gr}}_{n_{1}, n_{2}} \rightarrow \widetilde{\mathrm{Gr}}_{n_{1}}
$$

is a trivial bundle on $X_{n_{1}, n_{2}}^{n}$ (more importantly, by Lemma III.1.7 $\widetilde{G}(\widehat{\mathcal{K}})_{n_{1}, n_{2}}$ is the trivial $G(\widehat{\mathcal{O}})_{n_{2}}$-torsor over this set), so that on the open set $X_{n_{1}, n_{2}}^{n}$ we have a natural equivalence

$$
\left.\left.m_{n_{1}, n_{2}}^{*} \mathscr{G}_{n}\right|_{X_{n_{1}, n_{2}}^{n}} \cong\left(\mathscr{G}_{n_{1}} \widetilde{\otimes} \mathscr{G}_{n_{2}}\right)\right|_{X_{n_{1}, n_{2}}^{n}} .
$$

Similar considerations for more general $p$ establish such an equivalence with higher iterated twisted products.

Definition III.2.4. If $\mathscr{G}_{n}$ has a structure of factorizable equivariance, this structure is said to be strong if, for every $n$ and every partition $p$, the equivalence of (III.2) 
extends from the open set in $\widetilde{\mathrm{Gr}}_{p}$ over $X_{p}^{n}$ to all of $\widetilde{\mathrm{Gr}}_{p}$. (We will show that this is indeed merely a condition rather than additional data.)

There remains an additional compatibility to be imposed, which can be seen when considering Proposition I.3.9. Recall the quotient maps $q_{n}: G(\widehat{\mathcal{K}})_{n} \rightarrow \mathrm{Gr}_{n}$ and suppose that the $q_{n}^{*} \mathscr{G}_{n}$ were given a multiplicative structure for $G(\widehat{\mathcal{K}})_{n}$ together with a trivialization of that structure for $G(\widehat{\mathcal{O}})_{n}$. Then we have a $G(\widehat{\mathcal{O}})_{n^{-}}$ equivariance structure on $\mathscr{G}_{n}$. By Corollary III.1.11, we see that we have

$$
m^{*} \mathscr{G}_{n} \cong \mathscr{G}_{n} \widetilde{\nabla} \ldots \widetilde{\nabla} \mathscr{G}_{n}
$$

on Conv ${ }_{n}^{m}$, where the $m$ in $m^{*}$ is the map Conv ${ }_{n}^{m} \rightarrow \mathrm{Gr}_{n}$. By Proposition III.1.12. we see that the $\mathscr{G}_{n}$ are strongly factorizably equivariant. From the trivialization of $q_{n}^{*} \mathscr{G}_{n}$ as a multiplicative gerbe on $G(\widehat{\mathcal{O}})_{n}$, we deduce that the equivariance structure is trivialized on the unit section of $\mathrm{Gr}_{G, X^{n}}$, a condition we have not yet considered:

Definition III.2.5. The structure of factorizable equivariance on an sf gerbe $\mathscr{G}_{n}$ is said to be unital if $\mathscr{G}_{n}$ is sf equivariantly trivial on the unit sections on $\mathrm{Gr}_{G, X^{n}}$ (that is, its restriction to the trivial grassmannian $\mathrm{Gr}_{T, X^{n}}^{0, \ldots, 0}$ is trivial as a factorizably equivariant sf gerbe).

Having introduced the necessary concepts, the first consequence we will deduce is:

Theorem III.2.6. The following structures on an sf $A$-gerbe $\mathscr{G}_{n}$ on $\operatorname{Gr}_{G, X^{n}}$ are equivalent:

(1) A unital strongly factorizable equivariance for the action of $G(\widehat{\mathcal{O}})_{n}$.

(2) A factorizable multiplicative structure for the $q_{n}^{*} \mathscr{G}_{n}$ which is trivialized on $G(\widehat{\mathcal{O}})_{n}$.

Proof. We have already argued that the second point implies the first. To see the converse, we take $p=m \cdot n$ and consider the twisted product $\mathscr{G}_{n} \widetilde{\nabla} \ldots \widetilde{\nabla} \mathscr{G}_{n}$, on $\operatorname{Conv}_{n}^{m}$. It follows from the definition that when pulled back to $G(\widehat{\mathcal{K}})_{n} \times_{X^{n}}$ $\cdots \times_{X^{n}} G(\widehat{\mathcal{K}})_{n}$, this gerbe becomes $\left(q_{n}^{*} \mathscr{G}_{n}\right) \otimes \cdots \otimes\left(q_{n}^{*} \mathscr{G}_{n}\right)$. If the hypothesis of strong factorizability is satisfied, then we deduce from Corollary III.1.11 that there is an equivalence (here $m$ represents the multiplication map)

$$
m^{*}\left(q_{n}^{*} \mathscr{G}_{n}\right) \cong\left(q_{n}^{*} \mathscr{G}_{n}\right) \otimes \cdots \otimes\left(q_{n}^{*} \mathscr{G}_{n}\right) .
$$

It is clear from the expression Proposition III.1.10) and the associativity of tensor products that these isomorphisms are associative among themselves. This forms an associative multiplication on the $q_{n}^{*} \mathscr{G}_{n}$; the unit comes from that of the equivariance structure on $\mathscr{G}_{n}$.

We now turn to investigating the existence of strongly factorizable equivariance structures on sf gerbes. As usual, the first case to consider is that of a torus.

Proposition III.2.7. When $G=T$ is a torus, an sf $A$-gerbe $\mathscr{G}_{n}=\mathscr{T}_{n}$ on $\mathrm{Gr}_{T, X^{n}}$ has a unique structure of unital strongly factorizable equivariance for $T(\widehat{\mathcal{O}})_{n}$.

Proof. By Lemma III.1.14 and Definition II.3.1 we have for $p=(n-1,1)$ :

$$
m_{n-1,1}^{*} \mathscr{T}_{n}^{\lambda_{1}, \ldots, \lambda_{n-1}, \mu} \cong \mathscr{T}_{n-1}^{\lambda_{1}, \ldots, \lambda_{n-1}} \otimes \mathscr{T}_{1}^{\mu} \otimes \bigotimes_{i} \mathcal{O}\left(\Delta_{i n}\right)^{\log \kappa\left(\lambda_{i}, \mu\right)}
$$


where $\kappa$ is, as usual, the bilinear form defined by the sf gerbe. We must therefore show that the pullback of the latter product to $\widetilde{T}(\widehat{\mathcal{O}})_{n-1,1}^{\lambda_{1}, \ldots, \lambda_{n-1}} \times{ }_{X} \operatorname{Gr}_{1}^{\mu}$ is the same as $\mathscr{T}_{n-1}^{\lambda_{1}, \ldots, \lambda_{n-1}} \otimes \mathscr{T}_{1}^{\mu}$.

To do this, it is necessary and sufficient to only trivialize the second factor on pulling back, since the first factor pulls back to exactly the desired gerbe after using Lemma III.1.14 to identify the factorizations of the convolution product and its cover. The second factor is indeed trivialized after pulling back: first by Proposition III.1.17, identifying the product with $\mathscr{T}_{n-1,1}^{\log \kappa_{\mu}}$, and then by Corollary III.1.19, using the fact that

$$
\widetilde{T}(\widehat{\mathcal{O}})_{n-1,1}^{\lambda_{1}, \ldots, \lambda_{n-1}} \times_{X} \operatorname{Gr}_{1}^{\mu} \cong \widetilde{T}(\widehat{\mathcal{O}})_{n-1,1}^{\lambda_{1}, \ldots, \lambda_{n-1}}
$$

since $\mathrm{Gr}_{1}^{\mu} \cong X$.

The descent data of $\mathscr{T}_{n-1}^{\lambda_{1}, \ldots, \lambda_{n-1}} \otimes \mathscr{T}_{1}^{\mu}$ is the same as $T(\widehat{\mathcal{O}})_{1}$-equivariance for $\mathscr{T}_{1}^{\mu}$ (and, by agglomeration, all of $\mathscr{T}_{1}$ ); despite $T(\widehat{\mathcal{O}})_{1}$ acting trivially, this is not the trivial equivariance structure. Indeed, examining the proof of Lemma III.1.18, we see that in fact as we have defined it, the equivariance structure on $\mathscr{T}_{1}^{\mu}$, i.e., the equivalence between its pullbacks along the maps

$$
T(\widehat{\mathcal{O}})_{1} \times_{X} \operatorname{Gr}_{T, X}^{\mu} \underset{\mathrm{pr}}{\stackrel{a}{\longrightarrow}} \operatorname{Gr}_{T, X}^{\mu},
$$

with $a=\mathrm{pr}$, and therefore an autoequivalence of $\mathrm{pr}^{*} \mathscr{T}_{1}^{\mu}$, is given by the $A$-torsor $\mathcal{L}_{\kappa_{\mu}}$ pulled back to the first cartesian factor. This follows from any of the constructions for particular $\lambda_{i}$ and is thus, in particular, independent of their choice.

Therefore, the right-hand side of (III.4) fulfills the role of $\mathscr{T}_{n-1}^{\lambda_{1}, \ldots, \lambda_{n-1}} \widetilde{\nabla} \mathscr{T}_{1}^{\mu}$. Then by Lemma III.2.3 and induction on (III.4) $\mathscr{T}_{n}=m_{n-1,1}^{*} \mathscr{T}_{n}$ inherits a $T(\widehat{\mathcal{O}})_{n}$ equivariance structure as a twisted product, which by its definition is strongly factorizable; we have just argued that this structure is unique for $n=1$, and for higher $n$ the construction we have given is the only one possible, hence again unique. To see that it is unital, it suffices to show that $\mathscr{T}_{n}$ is sf-trivial on the components $\mathrm{Gr}_{n}^{0, \ldots, 0}$. These components form the grassmannian $\mathrm{Gr}_{1, X^{n}}$ of the trivial torus, and by Proposition II.3.6 it indeed has only the trivial sf gerbe on it.

The details of the above proof do not generalize to groups other than a torus both because Corollary III.1.19 fails for a nontorus and because we no longer have $\widetilde{\mathrm{Gr}}_{n_{1}, n_{2}} \cong \mathrm{Gr}_{n_{1}} \times \mathrm{Gr}_{n_{2}}$, so we proceed first by extending the equivariance structure and then showing separately that it is strongly factorizable.

Proposition III.2.8. Let $\mathscr{G}_{n}$ be the part of an sf $A$-gerbe on $\operatorname{Gr}_{G, X^{n}}$, and let $B$ be a fixed Borel subgroup of $G$ with semisimple quotient $T$; then the $T(\widehat{\mathcal{O}})_{n}$-equivariance structure of $\mathscr{T}_{n}$ on $\mathrm{Gr}_{T, X^{n}}$ extends uniquely to a $B(\widehat{\mathcal{O}})_{n}$-equivariance structure for $\mathscr{G}_{n}$.

Proof. The map $t: \operatorname{Gr}_{B, X^{n}} \rightarrow \mathrm{Gr}_{T, X^{n}}$ is biequivariant for the actions of $B(\widehat{\mathcal{O}})_{n}$ and $T(\widehat{\mathcal{O}})_{n}$, respectively, (along the natural map $\left.B(\widehat{\mathcal{O}})_{n} \rightarrow T(\widehat{\mathcal{O}})_{n}\right)$, so $\mathscr{B}_{n}=t^{*} \mathscr{T}_{n}$ gets a $B(\widehat{\mathcal{O}})_{n}$-equivariance structure by Proposition I.3.14. In fact, since $N(\widehat{\mathcal{O}})$ is cohomologically trivial, this structure is unique.

We have thus shown that there is a unique equivalence between the pullbacks of $\mathscr{B}_{n}$, the restriction of $\mathscr{G}_{n}$ to $\mathrm{Gr}_{B, X^{n}}$, along the two maps

$$
B(\widehat{\mathcal{O}})_{n} \times_{X^{n}} \operatorname{Gr}_{B, X^{n}} \underset{\mathrm{pr}}{\stackrel{a}{\longrightarrow}} \operatorname{Gr}_{B, X^{n}}
$$


satisfying the requirements of Definition I.3.1. Now we consider the diagram

$$
B(\widehat{\mathcal{O}})_{n} \times_{X^{n}} \operatorname{Gr}_{G, X^{n}} \underset{\mathrm{pr}}{\stackrel{a}{\longrightarrow}} \operatorname{Gr}_{G, X^{n}}
$$

and the pullbacks of $\mathscr{G}_{n}$ itself. According to Proposition II.5.2, the components of $\mathrm{Gr}_{B, X^{n}}$ stratify $\mathrm{Gr}_{G, X^{n}}$, and by (III.5) we have isomorphisms $a^{*} \mathscr{G}_{n} \cong \operatorname{pr}^{*} \mathscr{G}_{n}$ on each one of them. First fix $\lambda_{1}, \ldots, \lambda_{n}$ and for $\mu_{1}, \ldots, \mu_{n}$ with $\mu_{i}=\lambda_{i}-\check{\alpha}_{j}$ for any simple coroots $\check{\alpha}_{j}$, consider the complement of

$$
\overline{\mathrm{Gr}}_{B, X^{n}}^{\mu_{1}, \ldots, \mu_{n}} \subset{\overline{\mathrm{Gr}_{B, X^{n}}}}_{\lambda_{1}, \ldots, \lambda_{n}} .
$$

It is open and its boundary is a Cartier divisor, so the equivalence of (III.5) has some order along it. This order is seen to be 1 by considering the transverse section

$$
1_{X^{n}} \times_{X^{n}} \operatorname{Gr}_{G, X^{n}} \subset B(\widehat{\mathcal{O}})_{X^{n}} \times_{X^{n}} \operatorname{Gr}_{G, X^{n}},
$$

where both pullbacks are canonically identified because the identity section acts trivially and $\mathscr{B}_{n}$ has the trivial equivariance structure for this trivial action by the identity axiom of Definition I.3.1. Thus, we have $a^{*} \mathscr{G}_{n} \cong \operatorname{pr}^{*} \mathscr{G}_{n}$ on $\overline{\operatorname{Gr}}_{B, X^{n}}^{\lambda_{1}, \ldots, \lambda_{n}}$ for any choice of coweights $\lambda_{i}$, and that these isomorphisms glue for the inclusions of these strata as the $\lambda_{i}$ increase. Thus, $\mathscr{G}_{n}$ is $B(\widehat{\mathcal{O}})_{n}$-equivariant, as desired.

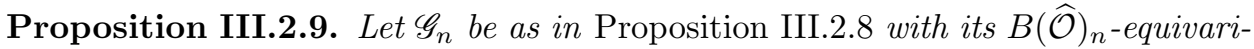
ance structure as constructed there. Then this structure extends uniquely to that of $G(\widehat{\mathcal{O}})_{n}$-equivariance.

Proof. The choice of Borel is arbitrary, so we also have a $B^{\mathrm{op}}(\widehat{\mathcal{O}})_{n}$-equivariance structure, where $B^{\text {op }}$ is the opposite Borel obtained by swapping the positive and negative roots. We have $B \cap B^{\mathrm{op}}=T$, and the "big cell" product $B^{\mathrm{op}} \cdot B=\mathrm{BC}$ is dense and open in $G$. The same hold of $B(\widehat{\mathcal{O}})_{n}$ as well. In the diagram

$$
B^{\mathrm{op}}(\widehat{\mathcal{O}})_{n} \times_{X^{n}} B(\widehat{\mathcal{O}})_{n} \times_{X^{n}} \operatorname{Gr}_{G, X^{n}} \underset{\mathrm{pr}}{\stackrel{a}{\longrightarrow}} \operatorname{Gr}_{G, X^{n}},
$$

where $a$ is obtained by the successive action of the two factors, both pullbacks of $\mathscr{G}_{n}$ are identified by their equivariance structures for the two Borel subgroups. The product of groups covers $\mathrm{BC}(\widehat{\mathcal{O}})_{n}$ as a $T(\widehat{\mathcal{O}})_{n}$-torsor and so this identification descends to $\operatorname{BC}(\widehat{\mathcal{O}})_{n} \times X^{n} \operatorname{Gr}_{G, X^{n}}$ via the $T(\widehat{\mathcal{O}})_{n}$-equivariance structure of $\mathscr{G}_{n}$.

Thus, the equivalence $a^{*} \mathscr{G}_{n} \cong \operatorname{pr}^{*} \mathscr{G}_{n}$ for the diagram

$$
G(\widehat{\mathcal{O}})_{n} \times_{X^{n}} \operatorname{Gr}_{G, X^{n}} \underset{\mathrm{pr}}{\stackrel{a}{\longrightarrow}} \operatorname{Gr}_{G, X^{n}}
$$

holds over a dense open subset of $G(\widehat{\mathcal{O}})_{n}$ and so has some order along the closed complement (which may indeed have codimension 1, as the example of $G=\mathrm{GL}_{2}$ shows). To see that it is trivial we need only show this on some transverse section, for example, the section

$$
G(\widehat{\mathcal{O}})_{n} \times_{X^{n}} \operatorname{Gr}_{G, X^{n}}^{0, \ldots, 0},
$$

in the notation of the upcoming Proposition IV.1.4 since $\operatorname{Gr}_{G, X^{n}}^{0, \ldots, 0}$, being equal to $G(\widehat{\mathcal{O}})_{n} / G(\widehat{\mathcal{O}})_{n} \cong X^{n}$, has a trivial action of $G(\widehat{\mathcal{O}})_{n}$ and on it, $\mathscr{G}_{n}=\mathscr{B}_{n}=\mathscr{T}_{n}$ has the trivial equivariance structure, by Proposition III.2.7.

Finally, we must show that the above (now globally) defined equivalence admits unique identity and associativity constraints making it an equivariance structure. The identity constraint is easy, as the restriction of $\phi=\left(a^{*} \mathscr{G}_{n} \cong \operatorname{pr}^{*} \mathscr{G}_{n}\right)$ to the identity section of $G(\widehat{\mathcal{O}})_{n} \times_{X^{n}} \mathrm{Gr}_{n}$ is the same as the restriction of the original 
$B(\widehat{\mathcal{O}})_{n}$-equivariance structure, which has such a constraint. For associativity, it is true a priori that the two sides of (I.4) differ by some $A$-torsor $\mathcal{T}$ on $G(\widehat{\mathcal{O}})_{n} \times_{X^{n}}$ $G(\widehat{\mathcal{O}})_{n} \times_{X^{n}} \mathrm{Gr}_{n}$. Since $\mathrm{Gr}_{n}$ is simply-connected, $\mathcal{T}$ descends to the product of groups, and therefore we can check that it is trivial by doing so when the last coordinate is fixed in $\operatorname{Gr}_{G, X^{n}}^{0, \ldots, 0}$, on which $\phi$ uniquely extends the trivial equivariance structure for $B(\widehat{\mathcal{O}})_{n}$, and is therefore itself trivial. We must also check that this trivialization of $\mathcal{T}$ satisfies the higher associativity condition, which is the same.

Theorem III.2.10. Any sf gerbe admits a unique unital strongly factorizable equivariance structure.

Proof. After the preceding propositions, it remains only to show that the $G(\widehat{\mathcal{O}})_{n^{-}}$ equivariance structures already constructed are strongly factorizable. We consider partitions $p=\left(n_{1}, n_{2}\right)$ for simplicity. Since the twisted product $\mathscr{G}_{n_{1}} \widetilde{\nabla} \mathscr{G}_{n_{2}}$ exists on $\widetilde{\mathrm{Gr}}_{n_{1}, n_{2}}$, and by factorizability and Proposition III.1.5 is identified with $m_{n_{1}, n_{2}}^{*} \mathscr{G}_{n_{1}+n_{2}}$ away from $X_{n_{1}, n_{2}}^{n}$, this identification has some order along $X_{n_{1}, n_{2}}^{n}$ which we must show is trivial. But as the $G(\widehat{\mathcal{O}})_{n_{i}}$-equivariance structures on the $\mathscr{G}_{n_{i}}$ extend the $T(\widehat{\mathcal{O}})_{n_{i}}$-equivariance structures on the $\mathscr{T}_{n_{i}}$, the twisted product restricted to $\widetilde{\mathrm{Gr}}_{T ; n_{1}, n_{2}}$ (in the obvious notation) is the same when using either equivariance and the claim follows from Proposition III.2.7.

\section{Part IV. Relative twisted geometric Satake equivalence}

In this part, we consider the following setup: $G$ is a connected, reductive algebraic group over $\mathbb{C}, k$ is any field of characteristic zero, and $\left\{\mathscr{G}_{n}\right\}$ is an sf $k^{*}$-gerbe. We consider only the unique strongly factorizable unital $G(\mathcal{\mathcal { O }})_{n}$-equivariance of Theorem III.2.10 on $\mathscr{G}_{n}$; using this, we construct the sheaf of categories of $G(\widehat{\mathcal{O}})_{n^{-}}$ equivariant perverse sheaves of $k$-vector spaces on $\operatorname{Gr}_{G, X^{n}}$ twisted by $\mathscr{G}_{n}$. Our main theorem, Theorem IV.8.3, describes this category in terms of the action of a certain "dual group" $\check{G}_{Q}$ (defined over $k$ and depending only on the quadratic form $\left.Q=Q\left(\mathscr{G}_{n}\right)\right)$ on the perverse sheaves on $X^{n}$, twisted by another gerbe $\mathscr{Z}_{n}$ over $\operatorname{Fact}\left(Z\left(\check{G}_{Q}\right)(k)\right)_{n}$, which was defined in Definition II.4.4 and (almost) appeared in Proposition II.4.9.

We will use the following notation: Perv ${ }_{S}$ will be the abelian category of perverse sheaves on the scheme $S$, and if $S$ has a gerbe $\mathscr{G}$, then $\operatorname{Perv}(\mathscr{G})_{S}$ will be the category of $\mathscr{G}$-twisted perverse sheaves. This notation is not specific to the affine grassmannian. However, we will write $\operatorname{Sph}\left(\mathscr{G}_{n}\right)$ for the "spherical", or $G(\widehat{\mathcal{O}})_{n^{-}}$ equivariant objects in $\operatorname{Perv}\left(\mathscr{G}_{n}\right)_{\mathrm{Gr}_{n}}$.

\section{IV.1. ORBITS IN THE AFFINE GRASSMANNIAN}

The last part of our study of the affine grassmannian concerns the orbits of the action of $G(\widehat{\mathcal{O}})_{n}$ on it. In order to produce perverse sheaves on these orbits we will need precise statements of their dimensions and the dimensions of related spaces.

Dimension of the orbits. We recall the absolute grassmannian

$$
\left.\mathrm{Gr}_{G}=G((t)) / G[t]\right]\left.\cong \mathrm{Gr}_{G, X}\right|_{x},
$$

where $x$ is any closed point of $X$ and $t$ is any choice of local coordinate there. We write $\mathcal{O}=\mathbb{C}[t]]$ and $\mathcal{K}=\mathbb{C}((t))$, so $\operatorname{Gr}_{G} \cong G(\widehat{\mathcal{K}}) / G(\widehat{\mathcal{O}})$; we will use the notation

$$
q: G(\widehat{\mathcal{K}}) \rightarrow \mathrm{Gr}_{G}
$$


for the projection onto this quotient.

Let $B$ be any Borel subgroup of $G$ and $T$ a maximal torus in $B$. Then $\operatorname{Gr}_{T} \cong \Lambda_{T}$ by Proposition II.1.2, while the components of $\mathrm{Gr}_{B}$ in $\mathrm{Gr}_{G}$, indexed by $\Lambda_{T}$, are the semi-infinite orbits. We write suggestively $t^{\lambda}$ for $\mathrm{Gr}_{T}^{\lambda}$, which is a single point identified with a coweight $\lambda \in \Lambda_{T}$, and sometimes identify it with an element of $G(\widehat{\mathcal{K}})$. As is customary in representation theory, we write

$$
2 \rho=\sum \alpha,
$$

the sum of all the positive roots of $G$, and use the partial ordering on weights or coweights in which positive elements are sums of positive roots.

Proposition IV.1.1. ([MV07]) The orbits of $G(\widehat{\mathcal{O}})$ in $\mathrm{Gr}_{G}$ correspond bijectively to $W$-orbits in $\Lambda_{T}$ according to the $t^{\lambda}$ they contain; equivalently, to the dominant coweights. For each dominant coweight $\lambda$, we have the closure relation

$$
\overline{\mathrm{Gr}}_{G}^{\lambda}=\bigcup_{\mu \leq \lambda} \operatorname{Gr}_{G}^{\mu}
$$

where the union runs over dominant $\mu$, and for any coweight $w_{0}(\lambda) \leq \mu \leq \lambda,\left(w_{0}\right.$ being the longest element of $W)$ the following intersection has pure dimension:

$$
\operatorname{dim}\left(\operatorname{Gr}_{G}^{\lambda} \cap \operatorname{Gr}_{B}^{\mu}\right)=\langle\rho, \lambda+\mu\rangle,
$$

Consequently, $\operatorname{dim} \overline{\mathrm{Gr}}_{G}^{\lambda}=\langle 2 \rho, \lambda\rangle$; in addition, when $\mu=w_{0}(\lambda)$, the intersection is the single point $\mathrm{Gr}_{T}^{\mu}$. In particular, $\mathrm{Gr}_{G}$ is stratified by finite-dimensional $G(\widehat{\mathcal{O}})$ orbits.

Connected with the $N(\widehat{\mathcal{K}})$-orbits is the following finiteness lemma for $N(\widehat{\mathcal{K}})$ itself.

Lemma IV.1.2. For any weight $\lambda$, let $N(\widehat{\mathcal{K}})_{\lambda}=t^{-\lambda} N(\widehat{\mathcal{O}}) t^{\lambda}$; then when $\mu-\lambda$ is dominant we have $N(\widehat{\mathcal{K}})_{\lambda} \subset N(\widehat{\mathcal{K}})_{\mu}$, and $N(\widehat{\mathcal{K}})=\bigcup_{\lambda} N(\widehat{\mathcal{K}})_{\lambda}$, the union taken over all dominant weights $\lambda$. If $Y \subset \mathrm{Gr}_{B}^{0}$ is any finite-dimensional subvariety, then $q^{-1}(Y) \subset N(\widehat{\mathcal{K}})_{\lambda}$ for some dominant $\lambda$.

Proof. First note that when $\lambda$ is antidominant, we have $N(\widehat{\mathcal{K}})_{\lambda} \subset N(\widehat{\mathcal{O}})$; indeed, if $n \in N(\widehat{\mathcal{O}})$, then for any highest-weight representation $V^{\mu}$ with a basis of weight vectors $e^{\nu}$ (not distinguishing between those of the same weight), we have

$$
n \cdot e^{\nu}=e^{\nu}+\sum_{\pi>\nu} n_{\nu, \pi} e^{\pi}, \quad \operatorname{ord}_{t} n_{\nu, \pi} \geq 0 .
$$

Thus, we have

$$
t^{-\lambda} n t^{\lambda} \cdot e^{\nu}=e^{\nu}+\sum_{\pi>\nu} t^{\langle\nu-\pi, \lambda\rangle} n_{\nu, \pi} e^{\pi}
$$

where $\langle\nu-\pi, \lambda\rangle \geq 0$ since $\lambda$ is antidominant. It follows, then, that if $\mu-\lambda$ is dominant, then we have

$$
N(\widehat{\mathcal{K}})_{\lambda}=t^{-\mu} N(\widehat{\mathcal{K}})_{\lambda-\mu} t^{\mu} \subset t^{-\mu} N(\widehat{\mathcal{O}}) t^{\mu}=N(\widehat{\mathcal{K}})_{\mu}
$$

as claimed.

As for the union, it suffices to establish it for the matrices of $N(\widehat{\mathcal{K}})$ acting on any faithful representation of $G$. Then we need only choose $\lambda$ large enough that $\langle\pi-\nu, \lambda\rangle+\operatorname{ord}_{t} n_{\nu, \pi} \geq 0$ for all weights $\nu, \pi$ of this representation, which since these differences are sums of positive roots we may accomplish for sufficiently large dominant coweights $\lambda$. 
Finally, if $Y \subset \operatorname{Gr}_{G}$ is finite-dimensional, then it intersects only finitely many $\operatorname{Gr}_{G}^{\lambda}$, but clearly the $q\left(N(\widehat{\mathcal{K}})_{\lambda}\right)$ intersect every one of these orbits. Since $N(\widehat{\mathcal{K}})$ is exhausted by the $N(\widehat{\mathcal{K}})_{\lambda}$, we must have $q^{-1}(Y)$ contained in one of them.

When the orbits are constructed as quotients of $G(\widehat{\mathcal{O}})$, their structure is simple:

Lemma IV.1.3. The stabilizer in $G(\widehat{\mathcal{O}})$ of $t^{\lambda}$ is connected and contains an algebraic subgroup $N^{\lambda}$, normal in $G(\widehat{\mathcal{O}})$, such that $G(\widehat{\mathcal{O}}) / N^{\lambda}$ is finite-dimensional.

Proof. Let $H$ be the stabilizer; we construct a surjection $H \rightarrow B$ and show that the kernel is unipotent (hence connected, since any finite group is reductive). This surjection is merely "evaluation at $t=0$ "; from the moduli description of Proposition II.5.1 we see that its image must only increase weights in representations of $G$, so it lies in $B$. Conversely, if we view $B \subset G(\widehat{\mathcal{O}})$, then this subgroup is a section of the evaluation map and for the same reason, preserves $t^{\lambda}$.

The kernel is a closed subgroup of the kernel in $G(\widehat{\mathcal{O}})$ of the evaluation map, so we need only show that the latter kernel $K$ is unipotent. Since $G(\widehat{\mathcal{O}})$ by definition has points

$$
G(\widehat{\mathcal{O}})(\operatorname{Spec} R)=G(\operatorname{Spec} R[t]])
$$

we also have "evaluation at $t^{n}=0$ " for all $n$, and thus a filtration of $K$ :

$$
K=K^{(1)} \supset K^{(2)} \supset \ldots
$$

by their kernels. In other words, $K^{(n)}$ is the subgroup of $G(\widehat{\mathcal{O}})$ of points specializing at $t^{n}=0$ to the identity in $G$, so that $K^{(1)} / K^{(2)} \cong \mathfrak{g}$, the tangent to $G$ at the identity. Thus, $K$ has a filtration by affine spaces, so it must be unipotent.

To obtain the second statement, we claim that for sufficiently large $n$, we have $K^{(n)} \subset H$. Since $K^{(n)}$ is the kernel of a specialization homomorphism from $G(\widehat{\mathcal{O}})$ and since each successive quotient $K^{(m)} / K^{(m+1)}$ is finite-dimensional, $G(\widehat{\mathcal{O}}) / K^{(n)}$ is a finite-dimensional algebraic group. The claim itself follows from Proposition II.5.1, in that once $n>\langle\lambda, \check{\alpha}\rangle$ for all simple coroots $\check{\alpha}$, for $g \in K^{(n)}$ both inclusions,

$$
\left.\langle v\rangle \otimes \mathcal{O}\left(X_{S} \backslash \bar{x}\right) \stackrel{\phi}{\hookrightarrow} V_{\mathcal{T}}\right|_{X_{S} \backslash \bar{x}},\left.\quad\langle v\rangle \otimes \mathcal{O}\left(X_{S} \backslash \bar{x}\right) \stackrel{g \phi}{\longrightarrow} V_{\mathcal{T}}\right|_{X_{S} \backslash \bar{x}}
$$

(in the notation there) have the same pole about $\bar{x}$.

By factorizability of $G(\widehat{\mathcal{O}})_{n}$ and $\operatorname{Gr}_{G, X^{n}}$, these results (or their products) hold in every fiber over $X^{n}$, leading to the evident generalization:

Proposition IV.1.4. The orbits of $G(\widehat{\mathcal{O}})_{n}$ in $\mathrm{Gr}_{G, X^{n}}$ are in bijection with $\Lambda_{T} / W$ (equivalently, the dominant coweights), depending on whether they contain the torus grassmannian components $\mathrm{Gr}_{T, X^{n}}^{\lambda_{1}, \ldots, \lambda_{n}}$ with fixed $\sum \lambda_{i}=\lambda$ modulo $W$. These orbits stratify $\mathrm{Gr}_{n}$.

If $\operatorname{Gr}_{B, X^{n}}^{\lambda}$ is the union of the same components of $\operatorname{Gr}_{B, X^{n}}$, then the intersection $\operatorname{Gr}_{G, X^{n}}^{\lambda} \cap \mathrm{Gr}_{B, X^{n}}^{\mu}$ is nonempty only for $w_{0}(\lambda) \leq \mu \leq \lambda$ and then has pure dimension $n+\langle\mu, \lambda\rangle$. The stabilizer of $\mathrm{Gr}_{T, X^{n}}^{\lambda}$ is connected and acts on $\operatorname{Gr}_{G, X^{n}}^{\lambda}$ by a finitedimensional quotient. 
Convolution diagrams. Since for any $x \in X$ we have $\left.\operatorname{Gr}_{G} \cong \operatorname{Gr}_{G, X}\right|_{x}$, we also have

$$
\left.\operatorname{Conv}_{1}^{n}\right|_{x}=\left.\left(G(\widehat{\mathcal{K}})_{X} \times^{G(\widehat{\mathcal{O}})_{X}} \ldots \times^{G(\widehat{\mathcal{O}})_{X}} \operatorname{Gr}_{G, X}\right)\right|_{x} \cong G(\widehat{\mathcal{K}}) \times^{G(\widehat{\mathcal{O}})} \ldots \times^{G(\widehat{\mathcal{O}})} \operatorname{Gr}_{G} .
$$

We will care only about the version where $n=2$ and call it simply $\operatorname{Conv}_{G}$ or, suggestively, $\mathrm{Gr}_{G} * \mathrm{Gr}_{G}$. We have the maps

$$
\text { pr, } m: \mathrm{Gr}_{G} * \mathrm{Gr}_{G} \rightarrow \mathrm{Gr}_{G}
$$

(projection is onto the first factor; $m$ comes from the action of $G(\widehat{\mathcal{K}})$ on $\operatorname{Gr}_{G}$ ). As in Proposition III.1.10, this convolution diagram is the moduli space of

$\left(\right.$ IV.1) $\operatorname{Conv}_{G}(S)=\left\{\left(\mathcal{T}_{1}, \mathcal{T}_{2}, \phi_{1}, \phi_{2}\right) \mid \begin{array}{c}\mathcal{T}_{i} \text { are } G \text {-torsors on } X, \\ \phi_{1} \text { is a trivialization of } \mathcal{T}_{1} \text { on } X \backslash \bar{x} \\ \phi_{2}: \mathcal{T}_{1} \rightarrow \mathcal{T}_{2} \text { is an isomorphism on } X \backslash \bar{x}\end{array}\right\}$

(for some fixed $x \in X$ ). The projection map pr becomes projection onto $\left(\mathcal{T}_{1}, \phi_{1}\right)$ and $m$ becomes projection onto $\left(\mathcal{T}_{2}, \phi_{2} \circ \phi_{1}\right)$.

Since every $G(\widehat{\mathcal{O}})$-orbit $\operatorname{Gr}_{G}^{\lambda}$ is (of course) $G(\widehat{\mathcal{O}})$-invariant, we can also form the diagram

$$
\operatorname{Conv}_{G}^{\lambda, \mu}=\mathrm{Gr}_{G}^{\lambda} * \mathrm{Gr}_{G}^{\mu}=q^{-1}\left(\mathrm{Gr}_{G}^{\lambda}\right) \times{ }^{G(\widehat{\mathcal{O}})} \operatorname{Gr}_{G}^{\mu}
$$

and write $\mathrm{pr}^{\lambda}, m^{\lambda, \mu}$ for the two maps above. As a twisted product, this convolution has the property that:

Lemma IV.1.5. $\mathrm{Gr}_{G}^{\lambda} * \mathrm{Gr}_{G}^{\mu}$ is a $\mathrm{Gr}_{G}^{\mu}$-bundle over $\mathrm{Gr}_{G}^{\lambda}$ and is thus smooth and irreducible of dimension $\langle 2 \rho, \lambda+\mu\rangle$.

We will require knowledge of the fibers of $m^{\lambda, \mu}$ in the following ways.

Proposition IV.1.6. The fiber $\left(m^{\lambda, \mu}\right)^{-1}\left(t^{\lambda+\mu}\right)$ consists of exactly one point $t^{\lambda} * t^{\mu}$.

Proof. We use the description $\operatorname{Conv}_{G}=G(\widehat{\mathcal{K}}) \times{ }^{G(\widehat{\mathcal{O}})} \operatorname{Gr}_{G}$ together with the identification $\operatorname{Gr}_{G}=G(\widehat{\mathcal{K}}) / G(\widehat{\mathcal{O}})$, and thus suppose we have a pair $(g, h) \in G(\widehat{\mathcal{K}}) \times G(\widehat{\mathcal{K}})$ such that

$$
g \in G(\widehat{\mathcal{O}}) t^{\lambda} G(\widehat{\mathcal{O}}), \quad h \in G(\widehat{\mathcal{O}}) t^{\mu} G(\widehat{\mathcal{O}}), \quad g h \in t^{\lambda+\mu} G(\widehat{\mathcal{O}}) .
$$

We must show that $g \in t^{\lambda} G(\widehat{\mathcal{O}}), h \in t^{\mu} G(\widehat{\mathcal{O}})$. Since in the pair $(g, h)$ we may pass factors in $G(\widehat{\mathcal{O}})$ across the comma, it suffices to show only the first; that is, we may write $g=u t^{\lambda}$ with $u \in G(\widehat{\mathcal{O}})$, and we want to show that $t^{-\lambda} u t^{\lambda} \in G(\widehat{\mathcal{O}})$. Let us also suppose that $h \in N(\widehat{\mathcal{O}}) t^{\mu} G(\widehat{\mathcal{O}})$. Thus, we have

$$
u t^{\lambda} n t^{\mu} G(\widehat{\mathcal{O}}) \subset t^{\lambda+\mu} G(\widehat{\mathcal{O}}),
$$

with $u \in G(\widehat{\mathcal{O}})$ and $n \in N(\widehat{\mathcal{O}})$. Rearranging, we get

$$
\left(t^{-\lambda} u t^{\lambda}\right) \in\left(t^{\mu} G(\widehat{\mathcal{O}}) t^{-\mu}\right) N(\widehat{\mathcal{O}}) .
$$

In any representation of $G$, the matrix of the left-hand side (in a weight basis partially ordered in the usual way) has all of its poles below the diagonal, while any matrix on the right-hand side has all of its poles above. Thus, the left-hand side is in fact in $G(\widehat{\mathcal{O}})$, as desired.

To remove the restriction on $h$, we use the fact that $\operatorname{Gr}_{G}^{\lambda}$ is the union of its intersections with $\operatorname{Gr}_{B^{w}}^{w(\lambda)}$, where $B^{w}=w B w^{-1}$. Since we have, equivalently, $\operatorname{Gr}_{G}^{\lambda}=$ $\operatorname{Gr}_{G}^{w(\lambda)}$ for any $w$, the proof works for suitable choice of $w$. 
For the next proposition, in the proof we will use the fact that for any subvariety $V \subset \operatorname{Gr}_{G}$ and any dominant coweight $\lambda$, we may construct the "convolution diagram" $V * \operatorname{Gr}_{G}^{\lambda} \subset \operatorname{Gr}_{G} * \operatorname{Gr}_{G}$ via

$$
V * \mathrm{Gr}_{G}^{\lambda}=q^{-1}(V) \times^{G(\widehat{\mathcal{O}})} \operatorname{Gr}_{G}^{\lambda}
$$

since by virtue of the pullback the first factor is a $G(\widehat{\mathcal{O}})$-torsor.

Proposition IV.1.7. For any dominant $\lambda \geq 0$, there is some dominant $\mu$ and an irreducible component in the fiber $\left(m^{\mu, \lambda}\right)^{-1}\left(t^{\mu}\right)$ of dimension at least $\langle\rho, \lambda\rangle=$ $\frac{1}{2} \operatorname{dim} \mathrm{Gr}_{G}^{\lambda}$.

Proof. To do this, we find such a component in a more amenable subspace. We claim that for $\mu$ sufficiently large, we have

$$
m^{-1}\left(t^{\mu}\right) \cap\left(\mathrm{Gr}_{B}^{\mu} * \mathrm{Gr}_{G}^{\lambda}\right) \subset m^{-1}\left(t^{\mu}\right) \cap\left(\mathrm{Gr}_{G}^{\mu} * \mathrm{Gr}_{G}^{\lambda}\right) .
$$

Granting this, the following additional equality is obtained by left-multiplying by $t^{\mu}$ :

$$
m^{-1}\left(t^{0}\right) \cap\left(\mathrm{Gr}_{B}^{0} * \mathrm{Gr}_{G}^{\lambda}\right)=m^{-1}\left(t^{\mu}\right) \cap\left(\mathrm{Gr}_{B}^{\mu} * \mathrm{Gr}_{G}^{\lambda}\right)
$$

and identifying $m^{-1}\left(t^{0}\right) \cong \operatorname{Gr}_{G}$ via pr, the former is identified with $\operatorname{Gr}_{B}^{0} \cap \mathrm{Gr}_{G}^{-w_{0}(\lambda)}$, which has pure dimension $\left\langle\rho,-w_{0}(\lambda)\right\rangle=\langle\rho, \lambda\rangle$ by Proposition IV.1.1 since $\lambda \geq 0$, as desired.

To prove the claim, we follow the proof of [BFGM02, Proposition 6.4]. We work with the description $\operatorname{Conv}_{G}=G(\widehat{\mathcal{K}}) \times{ }^{G(\widehat{\mathcal{O}})} \operatorname{Gr}_{G}$ and let $q: G(\widehat{\mathcal{K}}) \times G(\widehat{\mathcal{K}}) \rightarrow \operatorname{Conv}_{G}$ be the quotient map. Then $q^{-1}\left(\mathrm{Gr}_{B}^{\mu} * \mathrm{Gr}_{G}^{\lambda}\right)$ is identified with pairs $\left(t^{\mu} n u, v t^{\lambda} w\right)$ with $n \in N(\widehat{\mathcal{K}})$ and $u, v, w \in G(\widehat{\mathcal{O}})$, such that

$$
t^{\mu} n u v t^{\lambda} w=t^{\mu} x
$$

with $x \in G(\widehat{\mathcal{O}})$. In particular, we have $n^{-1} \in q^{-1}\left(\operatorname{Gr}_{G}^{\lambda}\right)$, or $n \in q^{-1}\left(\operatorname{Gr}_{B}^{0} \cap \operatorname{Gr}_{G}^{-w_{0}(\lambda)}\right)$, where the intersection is finite-dimensional. Applying Lemma IV.1.2 we have $n \in$ $t^{-\mu} N(\widehat{\mathcal{O}}) t^{\mu}$ for some $\mu$ depending only on $\lambda$, so $t^{\mu} n \in N(\widehat{\mathcal{O}}) t^{\mu} \subset q^{-1}\left(\mathrm{Gr}_{G}^{\mu}\right)$, as desired.

\section{IV.2. VANISHING CYCLES AND GLUING}

Before talking about gluing, we need to introduce a form of group action, related to factorizability, which is a key ingredient in the main theorem. (This is one of the things we will be gluing.) This was first given by Gaitsgory, Gai07, §2.5], and we state it for sections of any sheaf of $k$-linear categories.

Definition IV.2.1. Let $H$ be a group, $X$ a smooth curve, $Y \subset X^{n}$ be any subvariety, and let $\mathscr{F}$ be a sheaf of $k$-linear categories on $Y$. Then a factorizable action of $H$ on a section $s \in \mathscr{F}_{Y}$ is, simply, an action of $\left.\operatorname{Fact}(H)_{n}\right|_{Y}$ on $s$ (see Definition II.4.4). Explicitly, it is the following data: for every partition $p$ of $n$ into $m$ parts, an action of $H^{m}$ on $s$ over $Y \cap X_{p}^{n}$ such that, for any refinement $p^{\prime}$ of $p$ with $m^{\prime}$ parts, the action of $H^{m^{\prime}}$ on $s$ is the same as the corresponding diagonal action of $H^{m}$, over $Y \cap X_{p^{\prime}}^{n}$.

For example, when $n=2$, such an action is the data of an action of $H$ on $s$ as well as an action of $H \times H$ on $\left.s\right|_{Y \backslash \Delta}$, such that the restriction of the former to the latter set is the action of the diagonal in $H \times H$.

We will denote the sheaf of categories of perverse sheaves with a factorizable $H$-action by $\operatorname{FRep}_{n}(H)_{Y}$. 
One obvious fact that bears mention is that since $\operatorname{Fact}(H)_{n} \subset H^{n}$ by definition, any section with an action of $H^{n}$ automatically obtains a factorizable action. In addition, if $Y \subset X^{n}$ actually lies in one of the diagonals $\cong X^{n-1}$, then we have a natural equivalence $\operatorname{FRep}_{n-1}(H)_{Y} \cong \mathbf{F R e p}_{n}(H)_{Y}$ using (II.11).

We will also want to consider actions of an algebraic group on perverse sheaves, which requires a definition since perverse sheaves are not schemes. The following lemma and definition are basically trivial.

Lemma IV.2.2. Let $\mathbf{C}$ be a $k$-linear category for some field $k$ and let $x \in \mathbf{C}$; suppose $\operatorname{End}(x)$ is finite-dimensional. Then each $\operatorname{Aut}(x)$ has the natural structure of the rational points of an affine algebraic group defined over $k$.

Definition IV.2.3. Let $k$ be a field. An action of an algebraic $k$-group $H$ on an object in a $k$-linear category $\mathbf{C}$ is a regular homomorphism $H \rightarrow \operatorname{Aut}(x)$, the latter considered as an affine algebraic $k$-group. A map $f: x \rightarrow y$ of objects with an $H$ action is a map of $H$-actions if both maps $H \rightarrow \operatorname{Hom}(x, y)$ obtained by composition with $f$, the latter $k$-vector space considered as an affine $k$-scheme, are equal.

Note that if $x$ has an action of an algebraic $k$-group $H$, then it also has a literal group action of $H(k)$, though the converse is of course not true. If $\mathbf{C}$ is the category of finite-dimensional vector spaces, this definition is exactly the same as what is normally meant by the action of an algebraic group.

Lemma IV.2.4. Let $H$ be an algebraic $k$-group, let $\mathscr{F}$ be a sheaf of $k$-linear categories in which every $\operatorname{Aut}(s)$ is finite-dimensional, and let $\operatorname{Rep}(H, \mathscr{F})$ be the fibered category of sections of $\mathscr{F}$ with an $H$-action. Then $\operatorname{Rep}(H, \mathscr{F})$ is a sheaf of categories.

Proof. Let $\left\{U_{i}\right\}$ be an open cover containing all its finite intersections, let $\left\{s_{i}\right\}$ be sections of the $\operatorname{Rep}\left(H,\left.\mathscr{F}\right|_{U_{i}}\right)$, and let $\left\{\phi_{i j}:\left.s_{i}\right|_{U_{j}} \rightarrow s_{j}\right\}$ be isomorphisms whenever $U_{j} \subset U_{i}$. Then since $\mathscr{F}$ is a sheaf of categories, the $s_{i}$ glue to sections of $\mathscr{F}$, and we must show that the $H$-actions glue as well. Conjugation by $\phi_{i j}$ gives a map $\operatorname{Aut}\left(s_{i}\right) \rightarrow \operatorname{Aut}\left(s_{j}\right)$, and these maps form an inverse system indexed by the $U_{i j}$; by definition, $\operatorname{Aut}(s)=\lim \operatorname{Aut}\left(s_{i}\right)$, and since affine schemes admit all limits, this holds algebraically as well as set-theoretically. Since the $\phi_{i j}$ are maps of $G$-actions, the maps $H \rightarrow \operatorname{Aut}\left(s_{i}\right)$ are compatible with the limit and therefore assemble to a map of affine schemes $H \rightarrow \operatorname{Aut}(s)$, as desired.

It follows that when $H$ is an algebraic group, the categories $\operatorname{FRep}_{n}(H)$ form a sheaf of categories on $X^{n}$ (or on $Y \subset X^{n}$ ). Thus, we may also speak of twisting a factorizable action by a $\operatorname{Fact}(H(k))_{n}$-gerbe, and this will be important in what is to come.

Beilinson's gluing theorem. One of the most important technical properties of Perv $_{S}$ is the following: let $D=f^{-1}(0)$ be a principal Cartier divisor in $S$ (with $f: S \rightarrow \mathbb{A}^{1}$ ), with $j: U=S \backslash D \rightarrow S, i: D \rightarrow S$. For a perverse sheaf $\mathcal{M}$ on $U$, let $\Psi_{f}^{\mathrm{un}}(\mathcal{M})=R \psi_{f}^{\mathrm{un}}(\mathcal{M})[-1]$ be the (unipotent) nearby cycles of $\mathcal{F}$ along $f$, and for $\mathcal{F}$ on $S$, let $\Phi_{f}^{\text {un }}(\mathcal{F})=R \phi_{f}^{\text {un }}(\mathcal{F})[-1]$ be the vanishing cycles. The monodromy action on $\Psi_{f}^{\mathrm{un}}(\mathcal{M})$ is given by a unipotent automorphism $\mu$. The following theorem of Beilinson is discussed in the author's paper [Rei10].

Theorem. (Beilinson's gluing theorem) 
- Let $\mathcal{L}^{n}$ be the local system on $\mathbf{G}_{\mathbf{m}}$ of rank $n$ with monodromy given by a unipotent Jordan block. Then:

$$
i_{*} \Psi_{f}^{\mathrm{un}}(\mathcal{M}) \cong\left\{\begin{array}{l}
\lim _{n \rightarrow \infty} \operatorname{ker}\left(j_{!}\left(\mathcal{M} \otimes f^{*} \mathcal{L}^{n}\right) \rightarrow j_{*}\left(\mathcal{M} \otimes f^{*} \mathcal{L}^{n}\right)\right), \\
\lim _{\infty \leftarrow n} \operatorname{coker}\left(j_{!}\left(\mathcal{M} \otimes f^{*} \mathcal{L}^{n}\right) \rightarrow j_{*}\left(\mathcal{M} \otimes \mathcal{L}^{n}\right)\right), \\
\lim _{n \rightarrow \infty} i_{*} i^{*} j_{! *}\left(\mathcal{M} \otimes f^{*} \mathcal{L}^{n}\right)[-1],
\end{array}\right.
$$

with the monodromy acting via $\mathcal{L}^{n}$; consequently, $\Psi_{f}^{\text {un }}$ commutes with Verdier duality of perverse sheaves. In particular, the monodromy action on $\Psi_{f}^{\mathrm{un}}(\mathcal{M})$ is trivial if and only if $\Psi_{f}^{\mathrm{un}}(\mathcal{M})=i^{*} j_{!_{*}}(\mathcal{F})[-1]$, in which case $\Psi_{f}^{\mathrm{un}}$ depends only on $D$ and may be defined for non-principal Cartier divisors as well.

- Perv $v_{S}$ is equivalent to the gluing category of quadruples $\left(\mathcal{F}_{U}, \mathcal{F}_{D}, u, v\right)$, where $\mathcal{F}_{U}, \mathcal{F}_{D}$ are perverse sheaves on $U$ and $D$ and $\Psi_{f}^{\text {un }}\left(\mathcal{F}_{U}\right) \stackrel{u}{\rightarrow} \mathcal{F}_{D} \stackrel{v}{\rightarrow}$ $\Psi_{f}^{\mathrm{un}}\left(\mathcal{F}_{U}\right)$ are maps with $v \circ u=1-\mu$. The $\mathcal{F}_{D}$ term is given by the vanishing cycles functor $\Phi_{f}^{\mathrm{un}}(\mathcal{F})$, and Verdier duality acts on this data by dualizing all the terms, so that $u$ and $v$ are exchanged by duality.

We claim that this theorem holds also in the following circumstances:

\section{Proposition IV.2.5.}

(1) Let $Y$ be any scheme and $\mathscr{G}$ a $k^{*}$-gerbe on $Y$; then $\operatorname{Perv}(\mathscr{G})_{Y}$ has nearby cycles along any coordinate function $f$ and is equivalent to its gluing category.

(2) Let $\mathscr{G}$ be a $\operatorname{Fact}(H)_{n}$-gerbe on $Y$, and for $\mathcal{F} \in \mathscr{G} \otimes \operatorname{FRep}_{n}(H)_{Y}$, let $\Psi_{f}^{\text {un }}\left(\left.\mathcal{F}\right|_{U}\right)$ and $\Phi_{f}^{\text {un }}(\mathcal{F})$ be the perverse sheaves $\Psi_{f}^{\text {un }}\left(\left.\mathcal{F}\right|_{U}\right)$ and $\Phi_{f}^{\text {un }}(\mathcal{F})$ with their induced factorizable actions on $D$. Then $\mathscr{G} \otimes \operatorname{FRep}_{n}(H)_{Y}$ is equivalent to its gluing category.

(3) Let $Y$ be any scheme, $H$ any group scheme acting on $Y$, and $\mathscr{G}$ any $H$-equivariant gerbe on $Y$; then the category of $H$-equivariant objects in $\operatorname{Perv}(\mathscr{G})$ has nearby cycles and is equivalent to its gluing category.

Proof. Let $\mathbf{G}$ be the gluing category of perverse sheaves about a divisor $D$, let $V:$ Perv $\rightarrow$ G be the "vanishing cycles" functor associating a sheaf with its gluing data, and let $G$ be its inverse "gluing functor".

For the first claim, we construct the nearby and vanishing cycles of $\mathcal{F} \in \operatorname{Perv}(\mathscr{G})_{Y}$ by twisting those for ordinary perverse sheaves. To be precise, let $\mathscr{G}$ be a $k^{*}$-gerbe on $Y$ and let ${ }^{(x 2)} \mathscr{G}$ be its restrictions. Then we show that the functor $\Psi_{f}^{\text {un }}$ induces one

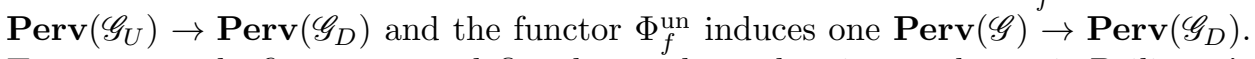
To construct the first, we may define the nearby cycles via $j_{\text {! }}$ and $j_{*}$ as in Beilinson's construction (taking the limit locally), and then by definition the gluing theorem is satisfied. To construct the second, we may employ the construction of vanishing cycles also given by Beilinson using these operations. Since these constructions (as shown in [Rei10]) agree with the usual ones for perverse sheaves, we have indeed produced twisted nearby and vanishing cycles functors.

For the second claim, by functoriality of $V$, the maps $u$ and $v$ respect the factorizable action and so $V(\mathcal{F})$ is in the gluing category of $\mathscr{G} \otimes \operatorname{FRep}_{n}(H)_{Y}$. Conversely, suppose we start with a quadruple $\mathcal{F}_{g}=\left(\mathcal{F}_{U}, \mathcal{F}_{D}, u, v\right) \in \mathbf{G}$ where both sheaves have factorizable actions, $\Psi_{f}^{\text {un }}\left(\mathcal{F}_{U}\right)$ is given the induced action, and $u: \Psi_{f}^{\text {un }}\left(\mathcal{F}_{U}\right) \rightarrow \mathcal{F}_{D}$ and $v$ preserve these actions. Then both $\mathcal{F}_{U}$ and $\mathcal{F}_{D}$ have $\operatorname{Fact}_{n}(H)$-actions, so $\mathcal{F}_{g}$ does. By functoriality, $G\left(\mathcal{F}_{g}\right)$ does as well. By Beilinson's 
theorem, $V G\left(\mathcal{F}_{g}\right) \cong \mathcal{F}_{g}$ and $G V(\mathcal{F}) \cong \mathcal{F}$ as perverse sheaves; we claim that these maps are isomorphisms of factorizable $H$-objects. For the first this is tautological from the construction of the $H$-action on a quadruple. For the second, we apply $V$ to both sides; then as just argued, the $H$-actions agree (taking $\mathcal{F}_{g}=V(\mathcal{F})$ ) and since $V$ is faithful, they agree without $V$ as well.

For the third claim, since the equivariant objects form a full subcategory, we only have to check that $V(\mathcal{F})$ is equivariant when $\mathcal{F}$ is and that $G\left(\mathcal{F}_{g}\right)$ is equivariant when the gluing data $\mathcal{F}_{g}$ is, which are tautological by functoriality.

Universally locally acyclic sheaves. The formalism of nearby cycles neatly complements the following concept of universal local acyclicity (ULA). Recall the Braverman-Gaitsgory formulation [BG02, §5.1] of the ULA condition:

Definition IV.2.6. We will say that a closed subscheme $i: Z \rightarrow S$ of pure codimension $d$ is smoothly embedded if $i ! \underline{k}_{S}=\underline{k}_{Z}[-2 d]$. For example, if $Y$ is smooth of pure dimension $d$, and $f: X \rightarrow Y$ is a map of schemes, then its graph $\Gamma_{f}: X \rightarrow X \times Y$ is smoothly embedded. For any map $g: T \rightarrow S$, and any complexes of sheaves $\mathcal{F}_{1}, \mathcal{F}_{2}$ on $S$, there is a natural map $g^{*} \mathcal{F}_{1} \otimes g^{!} \mathcal{F}_{2} \rightarrow g^{!}\left(\mathcal{F}_{1} \otimes \mathcal{F}_{2}\right)$ on $T$, obtained by adjunction from

$$
g_{!}\left(g^{*} \mathcal{F}_{1} \otimes g^{!} \mathcal{F}_{2}\right) \cong \mathcal{F}_{1} \otimes g_{!} g^{!} \mathcal{F}_{2} \rightarrow \mathcal{F}_{1} \otimes \mathcal{F}_{2}
$$

where the isomorphism is the projection formula. In the situation of a map $f: X \rightarrow$ $Y$, where $Y$ is smooth, if $\mathcal{F}_{X}, \mathcal{F}_{Y}$ are complexes on $X$ and $Y$, we take $T=Z=X$ (relative to the above notation), $S=X \times Y, g=i=\Gamma_{f}=(\mathrm{id}, f), \mathcal{F}_{1}=\mathcal{F}_{X} \otimes \mathcal{F}_{Y}$, and $\mathcal{F}_{2}=\underline{k}_{X \times Y}$; these considerations then give a natural map

$$
\mathcal{F}_{X} \otimes f^{*} \mathcal{F}_{Y} \rightarrow\left(\mathcal{F}_{X} \stackrel{!}{\otimes} f^{!} \mathcal{F}_{Y}\right)[2 \operatorname{dim} Y]
$$

where $\mathcal{F}_{1} \otimes ! \mathcal{F}_{2}=\mathbb{D}\left(\mathbb{D} \mathcal{F}_{1} \otimes \mathbb{D} \mathcal{F}_{2}\right)$ by definition, and $\mathcal{F}_{X}$ is said to be locally acyclic, or LA for $f$ if this is an isomorphism for all $\mathcal{F}_{Y}$, and universally locally acyclic, or ULA if this condition holds after any smooth base change of $Y$.

We introduce the superscript ULA, as in $\mathbf{S p h}^{\mathrm{ULA}}\left(\mathscr{G}_{n}\right)$, which we will only use with respect to the map $\operatorname{Gr}_{G, X^{n}} \rightarrow X^{n}$, for the ULA objects in these categories. ULA sheaves have some properties which are found in BG02.

Proposition IV.2.7. If $\mathcal{F}$ is $f$-ULA, then so is $\mathbb{D} \mathcal{F}$, and if $g: Z \rightarrow Y$ makes $Z$ a $Y$-scheme and $h: X \rightarrow Z$ is a proper map of $Y$-schemes, then $h_{*} \mathcal{F}$ is $g$-ULA. The ULA property is local in the smooth topology on $Y$ and if $\mathcal{F}$ is any complex of sheaves, then there exists a nonempty, Zariski-open subset of $Y$ over which $\mathcal{F}$ becomes ULA.

The following property of ULA perverse sheaves explains their importance.

Proposition IV.2.8. Let $D \subset Y$ be a smooth Cartier divisor and $E=f^{-1}(D)$ its preimage in $X, i: E \rightarrow X$ with complement $j: U \rightarrow X$. Then for any $\mathcal{F} \in$ $\operatorname{Perv}_{X}^{U L A}$, we have $j_{! *}\left(\left.\mathcal{F}\right|_{U}\right)=\mathcal{F}$. Furthermore, for any $U L A \mathcal{F}$ such that $j^{*} \mathcal{F}$ is perverse, monodromy acts trivially on the nearby cycles, so $\Psi_{E}^{\mathrm{un}}\left(\left.\mathcal{F}\right|_{U}\right)=i^{*} \mathcal{F}[-1]=$ $i^{!} \mathcal{F}[1]$. In particular, any such $\mathcal{F}$ is necessarily perverse and we have $\Phi_{E}^{\mathrm{un}}(\mathcal{F})=0$. Finally, when this happens, $i^{*} \mathcal{F}[-1]=\Psi_{E}^{\mathrm{un}}\left(\left.\mathcal{F}\right|_{U}\right)=i^{!} \mathcal{F}[1]$ is ULA for $\left.f\right|_{E}$.

Proof. Let $\underline{k}_{E}=i_{*} \underline{k}$ denote the constant sheaf supported at $E$; using the same notation for the inclusions of $D$ and its complement, $\underline{k}_{E}=f^{*} \underline{k}_{D}$. By definition, $i^{*} \mathcal{F}=\mathcal{F} \otimes \underline{k}_{E}$ and $i^{!} \mathcal{F}=\mathcal{F} \otimes ! \mathbb{D} \underline{k}_{E}$, and $j_{! *}\left(\left.\mathcal{F}\right|_{U}\right)$ is characterized by the 
property that it extends $\left.\mathcal{F}\right|_{U}$ and such that its $i^{*}$ and $i^{!}$have vanishing perverse 0 -cohomology; we show that this is true of $\mathcal{F}$. Note that since $D$ is smooth, $\mathbb{D} \underline{k}_{D}=\underline{k}_{D}[2(\operatorname{dim} Y-1)]$. By the ULA condition,

$$
\begin{aligned}
& i^{*} \mathcal{F}=\mathcal{F} \otimes f^{*} \underline{k}_{D}=\left(\mathcal{F} \stackrel{!}{\otimes} f^{!} \underline{k}_{D}\right)[2 \operatorname{dim} Y] \\
& =\left(\mathcal{F} \stackrel{!}{\otimes} f^{!} \mathbb{D} \underline{k}_{D}[2(1-\operatorname{dim} Y)]\right)[2 \operatorname{dim} Y]=i^{!} \mathcal{F}[2] .
\end{aligned}
$$

By [BBD82, Corollaire 4.1.10(ii)], $i^{*}$ exists only in perverse cohomologies -1 and 0 and $i^{!}$in degrees 0 and 1 ; thus, neither has any perverse cohomology in degree 0 , whence the claim.

To compute nearby cycles, we replace $Y$ with an open subset on which $D$ is defined by a single equation (since it will be irrelevant, we refer simply to $\Psi_{E}^{\mathrm{un}}$ ); let $\mathcal{L}^{n}$ denote the pullback to $Y$ from $\mathbf{G}_{\mathbf{m}}$ of the Jordan-block sheaf of Beilinson's theorem. Then

$$
\Psi_{E}^{\mathrm{un}}\left(\left.\mathcal{F}\right|_{U}\right)[1]=\lim _{n \rightarrow \infty} \operatorname{ker}\left(j_{!}\left(\left.\mathcal{F}\right|_{U} \otimes f^{*} \mathcal{L}^{n}\right) \rightarrow j_{*}\left(\left.\mathcal{F}\right|_{U} \otimes f^{*} \mathcal{L}^{n}\right)\right) .
$$

By the projection formula,

$$
j_{!}\left(\left.\mathcal{F}\right|_{U} \otimes f^{*} \mathcal{L}^{n}\right)=\mathcal{F} \otimes j_{!} f^{*} \mathcal{L}^{n}=\mathcal{F} \otimes f^{*} j_{!} \mathcal{L}^{n} .
$$

By the ULA condition for $\left.\mathcal{F}\right|_{U}$,

$$
\left.\mathcal{F}\right|_{U} \otimes f^{*} \mathcal{L}^{n}=\left.\mathcal{F}\right|_{U} \stackrel{!}{\otimes} f^{!} \mathcal{L}^{n}[2 \operatorname{dim} Y]
$$

and therefore by the projection formula again

$$
\begin{aligned}
j_{*}\left(\left.\mathcal{F}\right|_{U} \otimes f^{*} \mathcal{L}^{n}\right) & =j_{*}\left(\left.\mathcal{F}\right|_{U} \stackrel{!}{\otimes} f^{!} \mathcal{L}^{n}\right)[2 \operatorname{dim} Y] \\
& =\mathcal{F} \stackrel{!}{\otimes} j_{*} f^{!} \mathcal{L}^{n}[2 \operatorname{dim} Y]=\mathcal{F} \stackrel{!}{\otimes} f^{!} j_{*} \mathcal{L}^{n}[2 \operatorname{dim} Y]
\end{aligned}
$$

which by the ULA condition for $\mathcal{F}$ is just $\mathcal{F} \otimes f^{*} j_{*} \mathcal{L}^{n}$. Thus, the natural map $j_{!}\left(\left.\mathcal{F}\right|_{U} \otimes f^{*} \mathcal{L}^{n}\right) \rightarrow j_{*}\left(\left.\mathcal{F}\right|_{U} \otimes f^{*} \mathcal{L}^{n}\right)$ is the same as the natural map

$$
\mathcal{F} \stackrel{L}{\otimes} f^{*}\left(j_{!} \mathcal{L}^{n} \rightarrow j_{*} \mathcal{L}^{n}\right) .
$$

(We have inserted the ${ }^{L}$ to emphasize that tensor product is derived, though we have been neglecting this for the tensor product with locally free sheaves.) Let $C_{X}^{n}$ be the cone of this morphism, and let $C_{Y}^{n}$ be the cone of the map $j_{!} \mathcal{L}^{n} \rightarrow j_{*} \mathcal{L}^{n}$, so $C_{X}^{n}=\mathcal{F} \otimes^{L} f^{*} C_{Y}^{n}$. The $C_{Y}^{n}$, and consequently the $C_{X}^{n}$, form a sequence indexed by $n$, and we claim that the maps in this sequence are all isomorphisms for $C_{Y}^{n}$ for all $n$. Indeed, the perverse cohomologies of $C_{Y}$ compute the nearby cycles of the constant sheaf on $Y \backslash D$, which has no monodromy. Therefore $C_{X}^{n}$ is independent of $n$ as well, whence the result.

If $j^{*} \mathcal{F}$ is perverse when $\mathcal{F}$ is ULA, then we have just shown that $i^{*} \mathcal{F}[-1]=$ $\Psi_{E}^{\text {un }}\left(j^{*} \mathcal{F}\right)=i^{!} \mathcal{F}[1]$ are perverse, and therefore $\mathcal{F}$ is perverse. To show that $\Phi_{E}^{\mathrm{un}}(\mathcal{F})=0$, we consider the triangle

$$
i^{*} \mathcal{F} \rightarrow \Psi_{E}^{\mathrm{un}}\left(j^{*} \mathcal{F}\right)[1] \rightarrow \Phi_{E}^{\mathrm{un}}(\mathcal{F})[1] \rightarrow
$$

and substitute $\mathcal{F}=j_{!_{*}} j^{*} \mathcal{F}$ to obtain an isomorphism of the first two terms.

Finally, suppose that the isomorphisms $i^{*} \mathcal{F}[-1] \cong \Psi_{E}^{\text {un }}\left(\left.\mathcal{F}\right|_{U}\right) \cong i^{!} \mathcal{F}[1]$ hold. Then it follows that $\Psi_{E}^{\mathrm{un}}\left(\left.\mathcal{F}\right|_{U}\right)$ is ULA over $D$ : if $\mathcal{G}$ is a complex of sheaves on $D$, 
and if $i$ refers to the inclusions of both $D$ in $Y$ and $E$ in $X$, then we may apply the projection formula and its dual version,

$$
i_{!}\left(i^{*} \mathcal{F}_{1} \otimes \mathcal{F}_{2}\right) \cong \mathcal{F}_{1} \otimes i_{!} \mathcal{F}_{2}, \quad i_{*}\left(\mathcal{F}_{1} \stackrel{!}{\otimes} i^{!} \mathcal{F}_{2}\right) \cong i_{*} \mathcal{F}_{1} \stackrel{!}{\otimes} \mathcal{F}_{2},
$$

where in this case $i_{!}=i_{*}$ for a closed immersion, and write the chain of isomorphisms:

$$
\begin{aligned}
& i_{!}\left(i^{*} \mathcal{F} \otimes f^{*} \mathcal{G}\right) \cong \mathcal{F} \otimes i_{!} f^{*} \mathcal{G} \cong \mathcal{F} \otimes f^{*} i_{!} \mathcal{G} \\
& \cong\left(\mathcal{F} \otimes f^{!} i_{*} \mathcal{G}\right)[2 \operatorname{dim} Y] \cong\left(\mathcal{F} \otimes i_{*} f^{!} \mathcal{G}\right)[2 \operatorname{dim} Y] \cong i_{*}\left(i^{!} \mathcal{F} \otimes f^{!} \mathcal{G}\right)[2 \operatorname{dim} Y] \\
& \cong i_{*}\left(i^{*} \mathcal{F} \stackrel{!}{\otimes} f^{!} \mathcal{G}\right)[2(\operatorname{dim} Y-1)]
\end{aligned}
$$

where $\operatorname{dim} Y-1=\operatorname{dim} D$. Applying $i^{*}$ and shifting by -1 to get $\Psi_{E}^{\mathrm{un}}\left(\left.\mathcal{F}\right|_{U}\right)$ in place of the (co)restrictions, we have the ULA condition for $\Psi_{E}^{\mathrm{un}}\left(\left.\mathcal{F}\right|_{U}\right)$.

Corollary IV.2.9. If $\mathcal{F}$ is $U L A$ and perverse on $Y$ itself, then $\mathcal{F}[-\operatorname{dim} Y]$ is a locally constant sheaf ( $\mathcal{F}$ is lisse).

Proof. There is some Zariski open set $U$, which we assume to be the complement of a divisor $D$, such that $\left.\mathcal{F}\right|_{U}[-\operatorname{dim} Y]$ is a locally constant sheaf $\mathcal{L}$. By Proposition IV.2.8 the monodromy on $\Psi_{D}^{\text {un }}(\mathcal{L})$ is trivial, so the monodromy of $\mathcal{L}$ itself is trivial and thus $\mathcal{L}$ extends to a local system on $Y$ which we will also call $\mathcal{L}$. Since $Y$ is smooth, $\mathbb{D}(\mathcal{L}[\operatorname{dim} Y])=\mathcal{L}^{\prime}[\operatorname{dim} Y]$ with $\mathcal{L}^{\prime}$ again a locally constant sheaf, and so $\mathcal{L}[\operatorname{dim} Y]$ verifies the properties of the middle extension of $\left.\mathcal{F}\right|_{U}$, and hence is equal to $\mathcal{F}$.

Dévissage. The reason to use ULA sheaves is that every perverse sheaf is "generically" ULA by Proposition IV.2.7. In combination with Beilinson's vanishing cycles gluing, this allows us to reduce most theorems about perverse sheaves on $\mathrm{Gr}_{n}$ to ULA sheaves, for which Proposition IV.2.8 is a powerful tool. We prepare to state the theorem establishing such a principle by making a few meta-definitions.

Definition IV.2.10. Let $Y$ be any scheme, $\mathscr{F}$ a sheaf of abelian categories on $Y$. We will say that it admits gluing if it has pushforwards from any open subspace of $Y$ in the sense of Proposition I.2.1 as well as pushforwards with compact support, has an action of the tensor category of local systems on $Y$, and if Beilinson's theorem holds.

Let $Y_{1}, \ldots, Y_{n}$ be schemes and $\mathscr{F}_{1}, \ldots, \mathscr{F}_{n}$ be sheaves of abelian categories admitting gluing over the $Y_{i}$; let $\mathscr{F}_{n+1}$ be such a sheaf of categories over $Y_{n+1}=$ $Y_{1} \times \cdots \times Y_{n}$. We will say that a functor

$$
F: \mathscr{F}_{1} \times \cdots \times \mathscr{F}_{n} \rightarrow \mathscr{F}_{n+1}
$$

is horizontal if:

- It is a functor of categories fibered over $Y_{n+1}$, so it preserves restrictions to all open sets.

- It is exact in each argument and for local systems $\mathcal{L}_{1}, \ldots, \mathcal{L}_{n}$ on the $Y_{i}$ and any sections $s_{1}, \ldots, s_{n}$ in the $\mathscr{F}_{i}$, we have

$$
F\left(\mathcal{L}_{1} \otimes s_{1}, \ldots, \mathcal{L}_{n} \otimes s_{n}\right) \cong\left(\mathcal{L}_{1} \otimes \cdots \otimes \mathcal{L}_{n}\right) \otimes F\left(s_{1}, \ldots, s_{n}\right) .
$$


- For any Cartier divisors $D_{i} \subset Y_{i}$, denoting $j_{i}: U_{i}=Y_{i} \backslash D_{i} \rightarrow Y_{i}$ and setting $D_{n+1}=D_{1} \times \cdots \times D_{n}$ and $j: U_{n+1}=Y_{n+1} \backslash D_{n+1} \rightarrow Y_{n+1}$, the following natural maps

$$
\left(j_{n+1}\right) ! F \rightarrow F\left(\left(j_{1}\right) ! \times \cdots \times\left(j_{n}\right)_{!}\right) \quad F\left(\left(j_{1}\right)_{*} \times \cdots \times\left(j_{n}\right)_{*}\right) \rightarrow\left(j_{n+1}\right)_{*} F,
$$

obtained from the compatibility of $F$ with restriction to $U_{n+1}$, are isomorphisms.

We say that a natural transformation of horizontal functors is horizontal if it satisfies the analogous conditions.

Lemma IV.2.11. Given divisors as above, if $F$ is horizontal and the $D_{i}$ are principal, and if $\mathscr{F}_{n+1}$ admits gluing, we have

$$
\Psi_{D_{n+1}}^{\mathrm{un}} F \cong F\left(\Psi_{D_{1}}^{\mathrm{un}} \times \cdots \times \Psi_{D_{n}}^{\mathrm{un}}\right), \quad \Phi_{D_{n+1}}^{\mathrm{un}} F \cong F\left(\Phi_{D_{1}}^{\mathrm{un}} \times \cdots \times \Phi_{D_{n}}^{\mathrm{un}}\right),
$$

and $F$ is equal to the functor induced on nearby cycles gluing data by acting on each component of a quadruple. We will say that any functor for which these equations hold is compatible with nearby cycles. The same holds on natural transformations.

Proof. The first statement is a direct consequence of Beilinson's construction, after which the second statement is tautological.

Given the above definition, all of the sheaves of categories described in Proposition IV.2.5 admit gluing; important examples of horizontal functors will occur later in this part.

Lemma IV.2.12. Say that a functor is "left horizontal" if it satisfies all the hypotheses of being horizontal except that it only commutes with! extensions; likewise, $a$ "right horizonal" functor commutes with $*$ extensions. Suppose $g_{i}: X_{i} \rightarrow Y$ are maps of spaces and that $f: X_{1} \rightarrow X_{2}$ makes a commutative triangle; then between categories of constructible sheaves, $f_{!}$and $f^{*}$ are left horizontal while $f_{*}$ and $f^{!}$ are right horizontal. The composition of (left-, right-) horizontal functors is again (left-, right-) horizontal.

Proof. If $U \subset Y$, write $V_{i}=g_{i}^{-1}(U)$ and let $j_{i}$ be their inclusions; then $V_{1}=$ $f^{-1}\left(V_{2}\right)$. Clearly, $f_{*}\left(j_{1}\right)_{*}=\left(j_{2}\right)_{*} f_{*}\left(=\left(\left.f\right|_{V_{1}}\right)_{*}\right)$ and likewise for !'s, and that $f^{!}\left(j_{1}\right)_{*}=\left(j_{2}\right)_{*} f^{!}$and $f^{*}\left(j_{1}\right) !=\left(j_{2}\right)_{!} f^{*}$ are base change theorems. The last sentence is obvious.

Here is the precise definition of the problem we wish to solve using gluing.

Definition IV.2.13. Let $\mathscr{F}_{i}$ be as in Definition IV.2.10 and suppose $P_{i}$ are local properties of sections of these sheaves of categories, with $\mathscr{F}_{i}^{\prime}$ the full subsheaves of sections satisfying these properties. Let $S$ be a "structure" consisting of functors $\mathscr{F}_{1} \times \cdots \times \mathscr{F}_{n} \rightarrow \mathscr{F}_{n+1}$, natural transformations among them, and conditions on these data; we will write that $S$ is compatible with nearby cycles to mean that all these functors and transformations are. We say that specifying $S$ reduces to the $P_{i}$ if it suffices to do so after replacing the $\mathscr{F}_{i}$ by the $\mathscr{F}_{i}^{\prime}$ for $i \leq n$, and weakly reduces to the $P_{i}$ if we also require $\mathscr{F}_{n+1}^{\prime}$.

The following multipart metatheorem encapsulates a gluing argument used repeatedly throughout the rest of this work. 
Lemma IV.2.14. Let $Y_{i}$ be schemes which are noetherian spaces, $\mathscr{F}_{i}(i \leq n)$ sheaves of categories admitting gluing, and let $Y_{n+1}=\prod_{i} Y_{i}$, with $\mathscr{F}_{n+1}$ any sheaf of categories on it. Suppose that for each $i \leq n$, every section of $\mathscr{F}_{i}$ has property $P_{i}$ on a Zariski-dense open subset of $Y_{i}$. Then the following structures $S$, when compatible with nearby cycles, reduce to the $P_{i}$ when $\mathscr{F}_{n+1}$ admits gluing:

- Given a functor $F: \mathscr{F}_{1} \times \cdots \times \mathscr{F}_{n} \rightarrow \mathscr{F}_{n+1}, S$ is the condition that $F$ is exact.

- Given a pair of functors $F, G$ as above, $S$ is the structure of an isomorphism $t: F \rightarrow G$.

- $S$ is the structure of a functor $F$ as above.

In addition, suppose instead that $\mathscr{F}_{n+1}$ is a twisted derived category of constructible sheaves (thus, admitting nearby cycles) and $F$ is as above. Then the following structures $S$ also reduce to the $P_{i}$ :

- $S$ is the condition of $F$ taking values in perverse sheaves.

- If all the $\mathscr{F}_{i}$ are twisted derived categories, $S$ is the condition of $F$ being $t$-exact for the perverse $t$-structure.

Finally, suppose that all $\mathscr{F}_{i}(i \leq n+1)$ admit gluing, that sections of $\mathscr{F}_{n+1}$ generically have property $P_{n+1}$, and that $F$ is as above. Then the following structure $S$ weakly reduces to the $P_{i}$ :

- $S$ is the structure of a functor $G: \mathscr{F}_{n+1} \rightarrow \prod_{i \leq n} \mathscr{F}_{i}$ and isomorphisms $F G \rightarrow$ id and $G F \rightarrow$ id, realizing $F$ as an equivalence of categories.

Proof. We will use the following notation as standard in this proof: for each $i \leq n$, and for each principal Cartier divisor $D_{i} \subset Y_{i}$, let $\mathbf{G}_{i, D_{i}}$ be the "gluing category" consisting of nearby cycles gluing data $\left(s_{U_{i}}, s_{D_{i}}, u, v\right)$, where

$$
s_{U_{i}} \in \mathscr{F}_{i, U_{i}}^{\prime}, s_{D_{i}} \in \mathscr{F}_{i, D_{i}}, \Psi_{D_{i}}^{\mathrm{un}}\left(s_{U_{i}}\right) \stackrel{u}{\rightarrow} s_{D_{i}} \stackrel{v}{\rightarrow} \Psi_{D_{i}}^{\mathrm{un}}\left(s_{u_{i}}\right),
$$

with $1-v \circ u$ equal to the monodromy action on nearby cycles; here we have written $\mathscr{F}_{i, D_{i}}$ for the subsheaf of sections supported on $D_{i}$ in the sense that their restriction to $U_{i}$ is zero. Note that $s_{U_{i}}$ is assumed to have property $P_{i}$ but $s_{D_{i}}$ is not.

Beilinson's gluing theorem gives a fully faithful embedding of each $\mathbf{G}_{i, D_{i}}$ into $\mathscr{F}_{i, Y}$ (likewise for any open subscheme of $Y$ ). By hypothesis on the $\mathscr{F}_{i}$, every section $s \in \mathscr{F}_{i, Y}$ has property $P_{i}$ generically, so, without loss of generality, on the complement of some Cartier divisor, and therefore locally lies in the union of the $\mathbf{G}_{i, D_{i}}$.

For the first three points, let $\mathbf{G}_{n+1, D_{n+1}}$ be the gluing category in which $s_{U_{i}}$ is not assumed to have property $P_{n+1}$; here $D_{n+1}=\prod_{i<n} D_{i}$ as in Definition IV.2.10. Since all of the above structures $S$ respect nearby cycles and are local on $Y_{n+1}$, the following strategy suffices to prove the lemma: we assume by noetherian induction that $S$ obtains for the $\left.\mathscr{F}_{i}\right|_{D_{i}}$ and by hypothesis that it obtains for the $\left.\mathscr{F}_{i}^{\prime}\right|_{U_{i}}$, and construct the structure $S$ on the $\mathbf{G}_{i, D_{i}}(i \leq n+1)$. Note that all functors and natural transformations are, by Lemma IV.2.11 given term-by-term on gluing data. Here are the proofs of the first three points:

- Morphisms of gluing data are termwise (provided that they form commutative squares with $u$ and $v$ ) and the abelian structure on a category of gluing data is termwise on such morphisms, so exactness of a functor given termwise on gluing data is determined termwise as well. 
- This is tautological since natural transformations are termwise on gluing data.

- This is tautological since functors are termwise on gluing data.

For the next two points, we need not (and cannot) use gluing at all, so we replace the $\mathbf{G}_{i, D_{i}}(i \leq n)$ with the full subsheaves of the $\mathscr{F}_{i}$ whose sections are in $\mathscr{F}_{i}^{\prime}$ when restricted to $U_{i}$ to which they would otherwise be equivalent. We continue to assume the noetherian induction hypothesis.

- Suppose we have $s_{i} \in \mathscr{F}_{i, Y}$ such that $\left.s_{i}\right|_{U_{i}} \in \mathscr{F}_{i, Y}^{\prime}$; then

$$
\left.F\left(s_{1}, \ldots, s_{n}\right)\right|_{U_{n+1}}=F\left(\left.s_{1}\right|_{U_{i}}, \ldots,\left.s_{n}\right|_{U_{n}}\right),
$$

where the latter expression is, by hypothesis, perverse. Thus, since nearby cycles preserve perversity, $\left.\Psi_{D_{n+1}}^{\text {un }} F\left(\left\{s_{i}\right\}\right)\right|_{U_{n+1}}$ is perverse. Since $F$ also respects vanishing cycles, we have $\Phi_{D_{i+1}}^{\text {un }} F\left(\left\{s_{i}\right\}\right)=F\left(\left\{\Phi_{D_{i}}^{\text {un }} s_{i}\right\}\right)$, which is perverse by noetherian induction. We now invoke the distinguished triangle

$$
\left.\Psi_{D_{n+1}}^{\mathrm{un}} F\left(\left\{s_{i}\right\}\right)\right|_{U_{n+1}} \stackrel{u}{\rightarrow} \Phi_{D_{n+1}}^{\mathrm{un}} F\left(\left\{s_{i}\right\}\right) \rightarrow i^{*} F\left(\left\{s_{i}\right\}\right) \rightarrow
$$

in which the first two terms are both perverse, and so the third term is in $\mathscr{F}_{n+1}^{\leqslant 0}$. By the dual triangle

$$
\left.i^{!} F\left(\left\{s_{i}\right\}\right) \rightarrow \Phi_{D_{n+1}}^{\mathrm{un}} F\left(\left\{s_{i}\right\}\right) \stackrel{v}{\rightarrow} \Psi_{D_{n+1}}^{\mathrm{un}} F\left(\left\{s_{i}\right\}\right)\right|_{U_{n+1}} \rightarrow
$$

and the same argument we also have $i^{!} F\left(\left\{s_{i}\right\}\right) \in \mathscr{F}_{n+1}^{\geqslant 0}$, so we conclude that $F\left(\left\{s_{i}\right\}\right)$ is perverse.

- The argument that $F$ is $t$-exact is exactly the same, since the first triangle shows that $F$ preserves nonpositive perversity and the second one shows that it preserves nonnegative perversity.

Finally, for the last point we return to the gluing argument but let $\mathbf{G}_{n+1, D_{n+1}}$ contain only those gluing data with $s_{U_{i+1}} \in \mathscr{F}_{n+1, U_{n+1}}^{\prime}$.

- We simply invoke the third and second points of this lemma to construct $G$ and the two isomorphisms.

This completes the proof.

Of course, the hypothesis that every section of $\mathscr{F}_{i}$ is locally isomorphic to one in $\mathscr{F}_{i}^{\prime}$ is satisfied by ULA objects in any of the sheaves of categories considered in Proposition IV.2.5, which also satisfy nearby cycles gluing.

\section{IV.3. Convolutions}

In this section we construct two kinds of convolution products: most generally, we construct an "outer" convolution,

$$
*_{o}: \operatorname{Sph}\left(\mathscr{G}_{n}\right) \times \operatorname{Sph}\left(\mathscr{G}_{m}\right) \rightarrow \operatorname{Sph}\left(\mathscr{G}_{n+m}\right),
$$

and more specifically, we construct an "inner" convolution on the individual ULA categories:

$$
*_{i}: \mathbf{S p h}^{\mathrm{ULA}}\left(\mathscr{G}_{n}\right) \times \mathbf{S p h}^{\mathrm{ULA}}\left(\mathscr{G}_{n}\right) \rightarrow \mathbf{S p h}^{\mathrm{ULA}}\left(\mathscr{G}_{n}\right) .
$$

So as to apply Lemma IV.2.14, we will work not only over products $X^{n}$ but over any subscheme $Y \subset X^{n}$. To keep things neat, we will state definitions and theorems only for $X^{n}$; they can always be augmented by replacing each copy of an $X^{n}$ by a subscheme $Y$, and for products $\mathrm{Gr}_{n} \times \mathrm{Gr}_{m}$ or twisted products $\widetilde{\mathrm{Gr}}_{p}$ with 
$p: n+m=(n)+(m)$, the base should be replaced by a product $Y \times Y^{\prime}$ with $Y^{\prime} \subset X^{m}$. This partition will arise frequently, so we will denote it simply $(n, m)$.

Inner and outer convolution. The following notion is explained much more elegantly in [Gin95, 3.1]:

Definition IV.3.1. Let $\mathcal{F}_{1} \in \mathbf{S p h}\left(\mathscr{G}_{n}\right), \mathcal{F}_{2} \in \mathbf{S p h}\left(\mathscr{G}_{m}\right)$; then by equivariance we have a twisted pullback $\widetilde{\mathcal{F}}_{2} \in \mathbf{S p h}\left(\widetilde{\mathscr{G}}_{m}\right)$ on $\widetilde{\mathrm{Gr}}_{n, m}$. We define

$$
\mathcal{F}_{1} \widetilde{\otimes} \mathcal{F}_{2}=\operatorname{pr}_{n, m}^{*} \mathcal{F}_{1} \otimes \widetilde{\mathcal{F}}_{2} \in \operatorname{Sph}\left(\mathscr{G}_{n} \widetilde{\otimes} \mathscr{G}_{m}\right)
$$

on $\widetilde{\mathrm{Gr}}_{n, m}$, their twisted outer product as in Definition I.3.13 it is a $\left(\mathscr{G}_{n} \widetilde{\nabla} \mathscr{G}_{m}\right)$ twisted perverse sheaf by Corollary I.4.3, evidently $G(\widehat{\mathcal{O}})_{n+m}$-equivariant using Corollary III.1.13.

Recalling the construction of the twisted product as descending from the product

$$
\widetilde{G}(\widehat{\mathcal{K}})_{n, m} \times_{X^{m}} \mathrm{Gr}_{m}
$$

by $G(\widehat{\mathcal{O}})_{m}$-equivariance, and denoting $\mathrm{pr}_{n}, \mathrm{pr}_{m}$ the two maps from this space to $\mathrm{Gr}_{n}$ and $\mathrm{Gr}_{m}$, we see that $\mathcal{F}_{1} \widetilde{\otimes} \mathcal{F}_{2}$ descends from the outer tensor product

$$
\operatorname{pr}_{n}^{*} \mathcal{F}_{1} \otimes \operatorname{pr}_{m}^{*} \mathcal{F}_{2} \text {. }
$$

It must be noted that since $G(\widehat{\mathcal{O}})_{n}$ has infinite type over $X^{n}$, the concept of descent is problematic. This type of problem can be remedied by the following elementary fact:

Lemma IV.3.2. Let $\widetilde{H}$ be a group scheme, $\pi: \widetilde{H} \rightarrow H$ a quotient group scheme, $T$ a $\widetilde{H}$-torsor (over any scheme), and $S$ a scheme on which $\widetilde{H}$ acts through $H$. Then there is a natural isomorphism

$$
T \times{ }^{\widetilde{H}} S \rightarrow{ }^{1} \pi T \times{ }^{H} S .
$$

Proof. By definition, ${ }^{1} \pi T \times{ }^{H} S$ admits a map from ${ }^{1} \pi T \times S$ equalizing the action of $H$, and likewise, $T \times{ }^{\widetilde{H}} S$ admits a map from $T \times S$. We have a $\widetilde{H}$-equivariant map $T \rightarrow{ }^{1} \pi T$ and thus a map $T \times S \rightarrow{ }^{1} \pi T \times S$, so by composition a map $T \times S \rightarrow{ }^{1} \pi T \times{ }^{H} S$. By construction, it equalizes the action of $\widetilde{H}$, so descends to a map $T \times{ }^{\widetilde{H}} S \rightarrow{ }^{1} \pi T \times{ }^{H} S$. Locally this is just the map $\widetilde{H} \times \widetilde{H} S \rightarrow H \times{ }^{H} S$, which is an isomorphism, so it is an isomorphism.

By Proposition IV.1.4, every object of $\mathbf{S p h}\left(\mathscr{G}_{m}\right)$ is supported on a finite union of $G(\widehat{\mathcal{O}})_{m}$-orbits on which $G(\widehat{\mathcal{O}})_{m}$ acts through relatively finite-dimensional quotients. Let $\mathcal{F}$ be such an object, supported on the finite-dimensional $G(\widehat{\mathcal{O}})_{m}$-stable subscheme $S$ on which $G(\widehat{\mathcal{O}})_{m}$ acts through the finite-dimensional quotient $H$, and let $\mathcal{T}$ be the $H$-torsor over $\widetilde{G r}_{n}$ obtained from the $G(\widehat{\mathcal{O}})_{m}$-torsor $\widetilde{G}(\widehat{\mathcal{K}})_{n, m}$ by changing groups to $H$. Then $\widetilde{\mathcal{F}}$ lives on the subset $\widetilde{G}(\widehat{\mathcal{O}})_{n, m} \times G\left(\widehat{\mathcal{O}}_{m}\right) S \subset \widetilde{G r}_{n, m}$, which by Lemma IV.3.2 can be replaced by $\mathcal{T} \times{ }^{H} S$, which is a finite-dimensional smooth quotient of $\mathcal{T} \times S$. If $q$ is the quotient map, then it has relative dimension $\operatorname{dim} H$ and, by definition, we have

$$
q^{*} \widetilde{\mathcal{F}} \cong \operatorname{pr}_{S}^{*} \mathcal{F}
$$

and this is what we mean by the construction of the twisted outer product. Note that this definition does not depend on the particular choice of $H$. 
Since the equivariance structure on the $\mathscr{G}_{n}$ is strongly factorizable, in that we have an equivalence of equivariant gerbes

$$
m_{n, m}^{*} \mathscr{G}_{n+m} \cong \mathscr{G}_{n} \widetilde{\otimes} \mathscr{G}_{m},
$$

the following definition is possible:

Definition IV.3.3. For $\mathcal{F}_{1} \in \operatorname{Sph}\left(\mathscr{G}_{n}\right), \mathcal{F}_{2} \in \operatorname{Sph}\left(\mathscr{G}_{m}\right)$, let $\mathcal{F}_{1} \widetilde{\otimes} \mathcal{F}_{2}$ be the twisted product on $\widetilde{\mathrm{Gr}}_{n, m}$. Then the outer convolution is

$$
\mathcal{F}_{1} *_{o} \mathcal{F}_{2}=\left(m_{n, m}\right)_{*}\left(\mathcal{F}_{1} \widetilde{\otimes} \mathcal{F}_{2}\right) .
$$

When $n=m$, we define the inner convolution as:

$$
\mathcal{F}_{1} *_{i} \mathcal{F}_{2}=i_{\Delta}^{*}\left(\mathcal{F}_{1} *_{o} \mathcal{F}_{2}^{\prime}\right)[-n]
$$

where $\Delta \subset X^{n} \times X^{n}$ is the diagonal embedding of $X^{n}$. Equivalently, if we replace $\mathcal{F}_{1} \widetilde{\otimes} \mathcal{F}_{2}$ by its restriction to $\operatorname{Conv}_{n}^{2}$, then by proper base change we have $\mathcal{F}_{1} *_{i} \mathcal{F}_{2}=$ $\left(m_{n}\right)_{*}\left(\mathcal{F}_{1} \widetilde{\otimes} \mathcal{F}_{2}\right)$ in this sense. A priori these exist only in the twisted derived category.

It is also possible to make the following definition that more intuitively resembles the concept of convolution. We write

$$
q_{n}: G(\widehat{\mathcal{K}})_{n} \rightarrow \mathrm{Gr}_{n}, \quad q_{n, n}: G(\widehat{\mathcal{K}})_{n} \times{ }^{G(\widehat{\mathcal{O}})_{n}} G(\widehat{\mathcal{K}})_{n} \rightarrow \mathrm{Conv}_{n}^{2},
$$

where the first is the quotient map of Lemma III.1.3 and the second is that of Definition III.1.9, Multiplication in $G(\widehat{\mathcal{K}})_{n}$ descends along the quotient to give a map

$$
m_{n}^{\prime}: G(\widehat{\mathcal{K}})_{n} \times{ }^{G(\widehat{\mathcal{O}})_{n}} G(\widehat{\mathcal{K}})_{n} \rightarrow G(\widehat{\mathcal{K}})_{n} .
$$

Then there are two inner convolution products

$$
\mathcal{F}_{1}^{\prime} *_{*}^{\prime} \mathcal{F}_{2}^{\prime}=\left(m_{n}^{\prime}\right)_{*}\left(\mathcal{F}_{1}^{\prime} \widetilde{\otimes} \mathcal{F}_{2}^{\prime}\right), \quad \mathcal{F}_{1}^{\prime} *_{!}^{\prime} \mathcal{F}_{2}^{\prime}=\left(m_{n}^{\prime}\right)_{!}\left(\mathcal{F}_{1}^{\prime} \widetilde{\otimes} \mathcal{F}_{2}^{\prime}\right), \quad\left(\mathcal{F}_{i}^{\prime} \in \operatorname{Perv}\left(q_{n}^{*} \mathscr{G}_{n}\right)\right),
$$

where $\mathcal{F}_{1}^{\prime} \widetilde{\otimes} \mathcal{F}_{2}^{\prime}$ is the descent of $\mathcal{F}_{1}^{\prime} \otimes \mathcal{F}_{2}^{\prime}$ along the quotient.

In general, these operations are in fact as nice as one could want.

\section{Proposition IV.3.4.}

(1) In general, $\mathcal{F}_{1} *_{o} \mathcal{F}_{2} \in \operatorname{Sph}\left(\mathscr{G}_{n+m}\right)$, and if the $\mathcal{F}_{i}$ are $U L A$, we have the following identity, called the "fusion product":

$$
\mathcal{F}_{1} *_{o} \mathcal{F}_{2}=\left.j_{! *}\left(\mathcal{F}_{1} \otimes \mathcal{F}_{2}\right)\right|_{U}
$$

where $j$ is the inclusion of $U=X_{n, m}^{n+m}$ and we have used the factorization isomorphism $\mathrm{Gr}_{n+m} \cong \mathrm{Gr}_{n} \times \mathrm{Gr}_{m}$ on $U$; both inner and outer convolution are again ULA. Furthermore, both convolutions are exact.

(2) For both convolutions there are associativity constraints $\mathcal{F}_{1} *\left(\mathcal{F}_{2} * \mathcal{F}_{3}\right) \cong$ $\left(\mathcal{F}_{1} * \mathcal{F}_{2}\right) * \mathcal{F}_{3}$, and when $\mathcal{F}_{1}, \mathcal{F}_{2} \in \mathbf{S p h}^{U L A}\left(\mathscr{G}_{n}\right)$, there is a commutativity constraint $\mathcal{F}_{1} *_{i} \mathcal{F}_{2} \cong \mathcal{F}_{2} *_{i} \mathcal{F}_{1}$.

(3) We have

$$
q_{n}^{*}\left(\mathcal{F}_{1} *_{i} \mathcal{F}_{2}\right) \cong q_{n}^{*} \mathcal{F}_{1} *_{*}^{\prime} q_{n}^{*} \mathcal{F}_{2} \cong q_{n}^{*} \mathcal{F}_{1} *_{!}^{\prime} q_{n}^{*} \mathcal{F}_{2} \stackrel{\text { def }}{=} q_{n}^{*} \mathcal{F}_{1} *_{i}^{\prime} q_{n}^{*} \mathcal{F}_{2}
$$


Proof. We note that $\mathcal{F}_{1} *_{o} \mathcal{F}_{2}$ is equivariant by general nonsense. To show that convolution is exact and respects perversity, we use Lemma IV.2.14 for the functor $\operatorname{Sph}\left(\mathscr{G}_{n}\right) \times \mathbf{S p h}\left(\mathscr{G}_{m}\right) \rightarrow \mathbf{S p h}\left(\mathscr{G}_{n+m}\right)$. All three categories admit gluing over, respectively, $X^{m}, X^{n}$, and $X^{n+m}$, and convolution is compatible with nearby cycles (Lemma IV.2.11). Indeed, $\left(m_{n, m}\right)_{*}=\left(m_{n, m}\right)$ ! is a proper pushforward and, by Lemma IV.2.12, therefore, horizontal, while the outer tensor product (and therefore twisted outer product, since by [BBD82, Proposition 4.2.5] smooth pullbacks are faithful) is clearly horizontal. Thus, we suppose that $\mathcal{F}_{1}$ and $\mathcal{F}_{2}$ are both ULA.

Since the ULA condition is local in the smooth topology and stable under outer tensor products, the twisted product $\mathcal{F}_{1} \widetilde{\otimes} \mathcal{F}_{2}$ is ULA; since $m_{n, m}$ is proper, by Proposition IV.2.7 the pushforward $\mathcal{F}_{1} *_{o} \mathcal{F}_{2}$ is ULA, and therefore we have by Proposition IV.2.8

$$
\mathcal{F}_{1} *_{o} \mathcal{F}_{2}=\left.j_{! *}\left(\mathcal{F}_{1} *_{o} \mathcal{F}_{2}\right)\right|_{U}=\left.j_{! *}\left(\mathcal{F}_{1} \otimes \mathcal{F}_{2}\right)\right|_{U},
$$

using the identification of $\widetilde{\mathrm{Gr}}_{n, m}$ with $\mathrm{Gr}_{n} \times \mathrm{Gr}_{m}$ over $U$ (Proposition III.1.5) and the fact that $m_{n, m}$ is an equality there.

To analyze inner convolution, we apply $i_{\Delta}^{*}[-n]$ by applying successive $i_{\Delta_{i j}}^{*}[-1]$, where the diagonals $\Delta_{i j}$ are chosen so that they intersect in $\Delta$. By Proposition IV.2.8, each successive restriction is an application of nearby cycles and preserves perversity and the ULA property. We obtain the commutativity constraint on $*_{i}$ by swapping the first and last set of $n$ coordinates in $X^{2 n}$ before restricting, obtaining an isomorphism

$$
\left.\left.\mathrm{sw}^{*} j_{! *}\left(\mathcal{F}_{1} \otimes \mathcal{F}_{2}\right)\right|_{U} \cong j_{! *}\left(\mathcal{F}_{2} \otimes \mathcal{F}_{1}\right)\right|_{U} .
$$

Since $\Delta$ is invariant under the swapping map, both sides become equal to an inner convolution when we apply $i_{\Delta}^{*}$, as desired.

Finally, to obtain the associativity constraint it suffices, again by Lemma IV.2.14 (applied to the two functors $\left(\mathcal{F}_{1} *_{o} \mathcal{F}_{2}\right) *_{o} \mathcal{F}_{3}$ and $\mathcal{F}_{1} *_{o}\left(\mathcal{F}_{2} *_{o} \mathcal{F}_{3}\right)$, which are both compatible with nearby cycles), to do so for ULA sheaves. Since both orders of association are isomorphic to

$$
\left.j_{! *}\left(\mathcal{F}_{1} \otimes \mathcal{F}_{2} \otimes \mathcal{F}_{3}\right)\right|_{U}
$$

with $U \subset X^{n+m+l}$ the open set where none of the first $n$, next $m$, and last $l$ coordinates are equal, for ULA sheaves associativity follows from that of the tensor product. This isomorphism is obviously compatible with nearby cycles. By restriction, we also obtain associativity for inner convolution.

For (3), it is enough to observe that the "multiplication" map $m_{n, m}$ on the twisted grassmannian descends from $m_{n, m}^{\prime}$ using Corollary III.1.11 and Proposition III.1.12, and that convolution product of twisted sheaves on $G(\mathcal{K})_{n}$ is possible via the construction (I.7) using the sf multiplicative structure Theorem III.2.6 The ! and $*$ versions are equal since $m_{n}^{\prime}$ is the base change of the proper map $m_{n}$.

Rigidity of inner convolution. Finally, the inner convolution operation defines the structure of a rigid tensor category on $\mathbf{S p h}{ }^{\mathrm{ULA}}\left(\mathscr{G}_{n}\right)$. We will need the following generalization of the unit sections of Theorem III.2.10, there are closed immersions $i_{n, m ; 1}: \mathrm{Gr}_{n} \times X^{m} \rightarrow \mathrm{Gr}_{n+m}$ for every $n, m$, corresponding to the data

$$
((\vec{x}, \mathcal{T}, \phi), \vec{y}) \mapsto\left(\vec{x} \cup \vec{y}, \mathcal{T},\left.\phi\right|_{X_{S} \backslash(\bar{x} \cup \bar{y})}\right)
$$


where as usual, $\mathcal{T}$ is a $G$-torsor on $X_{S}$ and $\phi$ is its trivialization on the complement of the graphs of the coordinates of $\vec{x}: S \rightarrow X^{n}$. In fact, these maps are the same as the restrictions of

$$
m: \widetilde{\mathrm{Gr}}_{n, m} \rightarrow \mathrm{Gr}_{n+m}
$$

to the subset $\mathrm{Gr}_{n} * \mathrm{Gr}_{m}^{0}$, in the notation of Section IV.1, so that $i_{n, m ; 1}^{*} \mathscr{G}_{n+m}$ and $\mathscr{G}_{n} \cong \mathscr{G}_{n} \widetilde{\otimes}\left(\mathscr{G}_{n} \mid \mathrm{Gr}_{G}^{0}\right)$ are naturally equivalent. Likewise, we have maps $i_{n, m ; 2}$ inserting $X^{n}$ in the first $n$ coordinates and $\mathrm{Gr}_{m}$ in the last $m$.

Proposition IV.3.5. There is an element $1_{n} \in \mathbf{S p h}^{U L A}\left(\mathscr{G}_{n}\right)$ which is a unit for $*_{o}$ in the sense that for $\mathcal{F}_{1} \in \mathbf{S p h}\left(\mathscr{G}_{n}\right)$ and $\mathcal{F}_{2} \in \mathbf{S p h}\left(\mathscr{G}_{m}\right)$, we have

$$
\mathcal{F}_{1} *_{o} 1_{m} \cong\left(i_{n, m ; 1}\right)_{*} \mathcal{F}_{1}, \quad 1_{n} *_{o} \mathcal{F}_{2} \cong\left(i_{n, m ; 2}\right)_{*} \mathcal{F}_{2} .
$$

The unit sheaf $1_{n}$ is also an identity for $*_{i}$. Each object $\mathcal{F} \in \mathbf{S p h}^{U L A}\left(\mathscr{G}_{n}\right)$ has a dual object $\mathcal{F}^{*}$, in that

$$
\operatorname{Hom}\left(\mathcal{F}_{1} *_{i} \mathcal{F}_{2}, \mathcal{F}_{3}\right) \cong \operatorname{Hom}\left(\mathcal{F}_{1}, \mathcal{F}_{2}^{*} *_{i} \mathcal{F}_{3}\right)
$$

we have $\left(\mathcal{F}^{*}\right)^{*} \cong \mathcal{F}$ and $\left(\mathcal{F}_{1} *_{i} \mathcal{F}_{2}\right)^{*} \cong \mathcal{F}_{2}^{*} *_{i} \mathcal{F}_{1}^{*}$.

Proof. We remark first that since $\mathscr{G}_{n}$ is naturally trivialized on the unit section $X^{n} \rightarrow \mathrm{Gr}_{n}$ by Theorem III.2.10 the ordinary perverse sheaf $\underline{k}[n]$ on $X^{n}$ is $\mathscr{G}_{n^{-}}$ twisted and ULA and so if we denote $u=i_{0, n ; 1}=i_{0, n ; 2}$, we have $1_{n}=u_{*}(\underline{k}[n]) \in$ $\mathbf{S p h}^{\mathrm{ULA}}\left(\mathscr{G}_{n}\right)$ because a closed immersion is proper (applying Proposition IV.2.7). Then the twisted product $\mathcal{F}_{1} \widetilde{\otimes} 1_{m}$ lives on the convolution diagram

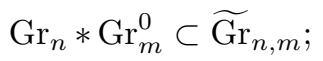

however, this subset is identified by the projection $\operatorname{pr}_{n}$ with $\mathrm{Gr}_{n} \times X^{m}$, and on it the twisted product is just the pullback of $\mathcal{F}_{1}$ from the first factor. Thus, its pushforward along $m$, which is $\mathcal{F}_{1} * 1_{m}$, is the same as $\left(i_{n, m ; 1}\right)_{*} \mathcal{F}_{1}$, as claimed; the same argument works for $1_{n} * \mathcal{F}_{2}$. To see that $1_{n}$ is an identity for $*_{i}$, just restrict to the appropriate diagonal, which converts $\widetilde{\mathrm{Gr}}_{n, n}$ into $\mathrm{Conv}_{n}^{2}$ by Proposition III.1.10)(1).

We define dualization in $\mathbf{S p h}^{\mathrm{ULA}}\left(\mathscr{G}_{n}\right)$ as follows. Let $\mathrm{Gr}_{n}^{0} \subset \mathrm{Gr}_{n}$ denote the image of the unit section $i_{0, n ; 1}=i_{0, n ; 2}$, and write $m$ : $\operatorname{Conv}_{n}^{2} \rightarrow \operatorname{Gr}_{n}$ for the multiplication map. Then $m^{-1}\left(\mathrm{Gr}_{n}^{0}\right) \cong \mathrm{Gr}_{n}$; indeed, the isomorphism is given by the projection pr: $\operatorname{Conv}_{n}^{2} \rightarrow \mathrm{Gr}_{n}$ and, explicitly, identifies data (recall Definition III.1.4)

$$
\left(\vec{x}, \mathcal{T}_{1}, \mathcal{T}_{2}, \phi_{1}, \phi_{2}\right) \cong(\vec{x}, \mathcal{T}, \phi)
$$

where the fact that the first datum is in $m^{-1}\left(\mathrm{Gr}_{n}^{0}\right)$ means, by definition, that $\phi_{2} \circ \phi_{1}$ extends to a trivialization of $\mathcal{T}_{2}$. Thus, we may identify $\phi_{1}=\phi_{2}^{-1}=\phi$ and $\mathcal{T}=\mathcal{T}_{1}$. Let $i: m^{-1}\left(\mathrm{Gr}_{n}^{0}\right) \rightarrow \operatorname{Conv}_{n}^{2}$; we set

$$
\mathcal{F}^{*}=\mathbb{D}\left(i^{*} \widetilde{\mathcal{F}}\right),
$$

where as always, $\widetilde{\mathcal{F}}$ is the twisted pullback of $\mathcal{F}$ to $\operatorname{Conv}_{n}^{2}$. To see that this is perverse, let $\operatorname{inv}_{n}: G(\widehat{\mathcal{K}})_{n} \rightarrow G(\widehat{\mathcal{K}})_{n}$ be the inversion map. Then by the above computation of $m^{-1}\left(\operatorname{Gr}_{n}^{0}\right)$, we have $q_{n}^{*} \mathcal{F}^{*} \cong \mathbb{D} \operatorname{inv}_{n}^{*} q_{n}^{*} \mathcal{F}$, where $q_{n}: G(\widehat{\mathcal{K}})_{n} \rightarrow \operatorname{Gr}_{n}$ is the quotient map, as usual; by descent, $\mathcal{F}^{*}$ is perverse. It also follows from the expression that $\left(\mathcal{F}^{*}\right)^{*} \cong \mathcal{F}$, since $\mathbb{D} \operatorname{inv}_{n}^{*}=\operatorname{inv}_{n}^{*} \mathbb{D}$. Note for later that $\operatorname{inv}_{n}^{*}=$ $\left(\operatorname{inv}_{n}\right)_{*}=\left(\operatorname{inv}_{n}\right)_{\text {! }}$; we denote their common value just by $\operatorname{inv}_{n}$. All of these claims follow from the fact that $\operatorname{inv}_{n}$ is an automorphism. 
We consider the identity $\left(\mathcal{F}_{1} *_{i} \mathcal{F}_{2}\right)^{*} \cong \mathcal{F}_{2}^{*} *_{i} \mathcal{F}_{1}^{*}$. To prove it, we use the expression of Proposition IV.3.4)(3)

$$
q_{n}^{*}\left(\mathcal{F}_{1} *_{i} \mathcal{F}_{2}\right) \cong q_{n}^{*} \mathcal{F}_{1} *_{i}^{\prime} q_{n}^{*} \mathcal{F}_{2} \cong\left(m_{n}^{\prime}\right)_{*, !}\left(q_{n}^{*} \mathcal{F}_{1} \widetilde{\otimes} q_{n}^{*} \mathcal{F}_{2}\right) .
$$

To apply the dualization formula above, we note that there is a well-defined automorphism

$$
\operatorname{inv}_{n, n}: G(\widehat{\mathcal{K}})_{n} \times^{G(\widehat{\mathcal{O}})_{n}} G(\widehat{\mathcal{K}})_{n} \rightarrow G(\widehat{\mathcal{K}})_{n} \times^{G(\widehat{\mathcal{O}})_{n}} G(\widehat{\mathcal{K}})_{n}, \quad(g, h) \mapsto\left(h^{-1}, g^{-1}\right)
$$

so that we have, for any $\mathcal{F}_{i} \in \operatorname{Perv}\left(q_{n}^{*} \mathscr{G}_{n}\right)$,

$$
\operatorname{inv}_{n, n}\left(\mathcal{F}_{1}^{\prime} *_{i}^{\prime} \mathcal{F}_{2}^{\prime}\right)=\operatorname{inv}_{n} \mathcal{F}_{2}^{\prime} *_{i}^{\prime} \operatorname{inv}_{n} \mathcal{F}_{1}^{\prime},
$$

using $\left(\operatorname{inv}_{n}\right)_{*}=\left(\operatorname{inv}_{n}\right) !=\operatorname{inv}_{n}$. Applying duality to both sides and taking $\mathcal{F}_{i}^{\prime}=$ $q_{n}^{*} \mathcal{F}_{i}$, we get the identity by descent.

Let $u: \mathrm{Gr}_{n}^{0} \rightarrow \mathrm{Gr}_{n}$ be the inclusion of the "unit" section, so $1_{n}=u_{*} \underline{k}[n]$. Then we have for any $\mathcal{F}_{1}, \mathcal{F}_{2} \in \mathbf{S p h}^{\mathrm{ULA}}\left(\mathscr{G}_{n}\right)$ :

$$
\begin{aligned}
& \operatorname{Hom}\left(\mathcal{F}_{1} *_{i} \mathcal{F}_{2}, 1_{n}\right)=\operatorname{Hom}\left(m_{*}\left(\operatorname{pr}^{*} \mathcal{F}_{1} \otimes \widetilde{\mathcal{F}}_{2}\right), 1_{n}\right) \\
& \quad=\operatorname{Hom}\left(\operatorname{pr}^{*} \mathcal{F}_{1} \otimes \widetilde{\mathcal{F}}_{2}, m^{!} 1_{n}\right)=\operatorname{Hom}\left(\operatorname{pr}^{*} \mathcal{F}_{1}, \mathcal{H o m}\left(\widetilde{\mathcal{F}}_{2}, m^{\prime} u_{*} \underline{k}[n]\right)\right) .
\end{aligned}
$$

But $m^{!} u_{*} \underline{k}[n]=i_{*} m^{!} \underline{k}[n]=i_{*} \mathcal{D}$, where $\mathcal{D}$ is the dualizing sheaf $m^{-1}\left(\operatorname{Gr}_{n}^{0}\right)$. Then the right operand is simply

$$
\mathcal{H o m}\left(\widetilde{\mathcal{F}}_{2}, i_{*} \mathcal{D}\right)=i_{*} \mathcal{H o m}\left(i^{*} \widetilde{\mathcal{F}}_{2}, \mathcal{D}\right)=i_{*} \mathbb{D}\left(i^{*} \widetilde{\mathcal{F}}_{2}\right)=i_{*} \mathcal{F}_{2}^{*} .
$$

Finally, then, we have

$$
\operatorname{Hom}\left(\mathcal{F}_{1} *_{i} \mathcal{F}_{2}, 1_{n}\right)=\operatorname{Hom}\left(\operatorname{pr}^{*} \mathcal{F}_{1}, i_{*} \mathcal{F}_{2}^{*}\right)=\operatorname{Hom}\left(i^{*} \operatorname{pr}^{*} \mathcal{F}_{1}, \mathcal{F}_{2}^{*}\right)=\operatorname{Hom}\left(\mathcal{F}_{1}, \mathcal{F}_{2}^{*}\right),
$$

as desired. If we have a third sheaf $\mathcal{F}_{3}$, then formally:

$$
\begin{aligned}
\operatorname{Hom}\left(\mathcal{F}_{1} *_{i} \mathcal{F}_{2}, \mathcal{F}_{3}\right) \cong \operatorname{Hom}\left(\mathcal{F}_{1} *_{i}\right. & \left.\mathcal{F}_{2},\left(\mathcal{F}_{3}^{*}\right)^{*}\right) \\
\cong \operatorname{Hom}\left(\left(\mathcal{F}_{1} *_{i} \mathcal{F}_{2}\right) *_{i}\right. & \left.\mathcal{F}_{3}^{*}, 1_{n}\right) \cong \operatorname{Hom}\left(\mathcal{F}_{1} *_{i}\left(\mathcal{F}_{2} *_{i} \mathcal{F}_{3}^{*}\right), 1_{n}\right) \\
& \cong \operatorname{Hom}\left(\mathcal{F}_{1},\left(\mathcal{F}_{2} *_{i} \mathcal{F}_{3}^{*}\right)^{*}\right) \cong \operatorname{Hom}\left(\mathcal{F}_{1}, \mathcal{F}_{2}^{*} *_{i} \mathcal{F}_{3}\right),
\end{aligned}
$$

as desired.

This definition of the dual (phrased differently) was given in Gin95, §2.4] and reprised in [MV07, (11.10)].

\section{IV.4. THE FIBER FUNCTOR}

In this section, we will produce a map from spherical sheaves on $\mathrm{Gr}_{G, X^{n}}$ to those on $\operatorname{Gr}_{T, X^{n}}$ which will turn out to retain all of the information about the former. We recall the fundamental diagram,

$$
\mathrm{Gr}_{G, X^{n}} \stackrel{b}{\leftarrow} \mathrm{Gr}_{B, X^{n}} \stackrel{t}{\rightarrow} \mathrm{Gr}_{T, X^{n}},
$$

corresponding to any choice of Borel subgroup $B \subset G$ and its quotient torus $T=$ $B / N$. We also recall the indexing of the irreducible components of $\operatorname{Gr}_{B, X^{n}}$ by $\Lambda_{T}^{n}$, which is the same as that of $\mathrm{Gr}_{T, X^{n}}$; the connected component containing $\mathrm{Gr}_{B, X^{n}}^{\lambda_{1}, \ldots \lambda_{n}}$ depends only on the sum of the $\lambda_{i}$. If $\mathscr{G}_{n}$ is an sf gerbe on $\operatorname{Gr}_{G, X}$, then we let $\mathscr{T}_{n}$ be the unique sf gerbe on $\operatorname{Gr}_{T, X^{n}}$ such that $t^{*} \mathscr{T}_{n} \cong b^{*} \mathscr{G}_{n}$, as in (II.19) 
We use the notation, standard in representation theory,

$$
2 \rho=\sum \alpha
$$

where the $\alpha$ 's run over all positive roots of $G$.

\section{The maximal fiber functor.}

Definition IV.4.1. The fiber functor $F_{n}: \operatorname{Sph}\left(\mathscr{G}_{n}\right) \rightarrow \mathscr{D}\left(\mathscr{T}_{n}\right)$ is either one of the following two functors (which we show are isomorphic):

- The functor $\left(F_{n}\right)_{\text {! }}^{*}$, where for $\mathcal{F} \in \mathbf{S p h}\left(\mathscr{G}_{n}\right)$, we let $\mathcal{F}^{\lambda}$ be the restriction of $b^{*} \mathcal{F}$ to the connected component $\operatorname{Gr}_{B, X^{n}}^{\lambda}$, and set

$$
\left(F_{n}\right)_{!}^{*}(\mathcal{F})^{\lambda}=t_{!}^{\lambda} \mathcal{F}^{\lambda}[\langle 2 \rho, \lambda\rangle]
$$

to be the component of $\left(F_{n}\right)_{!}^{*}(\mathcal{F})$ on $\operatorname{Gr}_{T, X^{n}}^{\lambda}$.

- The functor $\left(F_{n}\right)_{*}^{!}$, replacing $b^{*}$ with $b^{!}$and $t_{\text {! with }} t_{*}$.

We observe that for ULA sheaves, we have

$$
\left(F_{n}\right)_{!}^{*}(\mathcal{F})^{\lambda}=\mathbb{D}\left(F_{n}\right)_{*}^{!}(\mathbb{D} \mathcal{F})^{-\lambda}=\mathbb{D}\left(F_{n}\right)_{*}^{!}\left(\mathcal{F}^{*}\right)^{\lambda},
$$

and set $F=F_{*}^{!}$.

\section{Proposition IV.4.2.}

(1) Neither $\left(F_{n}\right)_{!}^{*}$ nor $\left(F_{n}\right)_{*}^{!}$depends on the choice of Borel or torus in $G$, and they are isomorphic.

(2) The functor $F_{n}$ is t-exact, faithful, and preserves the ULA property.

(3) There are natural isomorphisms

$$
\begin{aligned}
F_{n+m}\left(\mathcal{F}_{1} *_{o} \mathcal{F}_{2}\right) & \cong F_{n}\left(\mathcal{F}_{1}\right) *_{o} F_{m}\left(\mathcal{F}_{2}\right), \\
F_{n}\left(\mathcal{F}_{1} *_{i} \mathcal{F}_{2}\right) & \cong F_{n}\left(\mathcal{F}_{1}\right) *_{i} F_{n}\left(\mathcal{F}_{2}\right)
\end{aligned}
$$

(the latter when the sheaves are ULA) compatible with the associativity constraints.

Proof. For (1), note that alternate choices of $B$ and $T$ are obtained by conjugation; i.e., $B^{\prime}=g^{-1} B g, T^{\prime}=g^{-1} T g$ for some $g \in G(k)$. On the affine grassmannian, this means that the various possible $b$ are permuted by translation by $G(\mathbb{C}) \times X^{n} \subset$ $G(\widehat{\mathcal{O}})_{n}$, and so applied to spherical objects their pullbacks are the same. The identity $\left(F_{n}\right)_{!}^{*}=\left(F_{n}\right)_{*}^{!}$follows from [Bra03] (as in [MV07, Theorem 3.5]).

To show that it is faithful, the argument in [MV07] also works: if the support of $\mathcal{F} \in \operatorname{Sph}\left(\mathscr{G}_{n}\right)$ intersects $\operatorname{Gr}_{B, X^{n}}^{\lambda}$ for some $\lambda$ which is minimal with respect to the ordering of coweights by positive coroots, then this intersection is a single point in each fiber over $X^{n}$ and the stalk of $F_{n}(\mathcal{F})^{\lambda}$ is the stalk of $\mathcal{F}$ at that point.

To show that $F_{n}$ preserves the ULA property, we do a formal computation. For $\mathcal{G}$ any complex on $X^{n}$ and $f_{G}, f_{B}, f_{T}$ the structure maps $\operatorname{Gr}_{G, X^{n}} \rightarrow X^{n}$, etc., we have up to shifts and indexing:

$$
\begin{aligned}
\left(F_{n}\right)_{!}^{*}\left(\mathcal{F} \otimes f_{G}^{*} \mathcal{G}\right) & =t_{!} b^{*}\left(\mathcal{F} \otimes f_{G}^{*} \mathcal{G}\right)=t_{!}\left(b^{*} \mathcal{F} \otimes f_{B}^{*} \mathcal{G}\right)=t_{!}\left(b^{*} \mathcal{F} \otimes t^{*} f_{T}^{*} \mathcal{G}\right) \\
& =\left(t_{!} b^{*} \mathcal{F}\right) \otimes f_{T}^{*} \mathcal{G}=\left(F_{n}\right)_{!}^{*} \mathcal{F} \otimes f_{T}^{*} \mathcal{G}
\end{aligned}
$$

The crucial step was an application of the projection formula for $t$. An application of the dual projection formula $t_{*}\left(b^{!} \mathcal{F} \otimes ! t^{!} f_{T}^{!} \mathcal{G}\right)=\left(t_{*} b^{!} \mathcal{F}\right) \otimes ! f_{T}^{!} \mathcal{G}$ gives

$$
\left(F_{n}\right)_{*}^{!}\left(\mathcal{F} \stackrel{!}{\otimes} f_{G}^{!} \mathcal{G}\right)=\left(F_{n}\right)_{*}^{!} \mathcal{F} \stackrel{!}{\otimes} f_{T}^{!} \mathcal{G} .
$$


Since $\left(F_{n}\right)_{*}^{!}=\left(F_{n}\right)_{!}^{*}$, shifting this by $2 n$ and equating the left sides by the ULA property of $\mathcal{F}$, one concludes the equation of the ULA property for $F_{n}(\mathcal{F})$.

To show that $F_{n}$ is $t$-exact, we perform some reductions and invoke a few results that will be proven in later sections (they do not depend on this proposition). First, by Lemma IV.2.14, it suffices to show that it sends ULA perverse sheaves to perverse sheaves. Indeed, by Lemma IV.2.12, $F_{n}$ (as the composition both of a ! pullback and $*$ pushforward and a $*$ pullback and ! pushforward) is horizontal, hence compatible with nearby cycles by Lemma IV.2.11. Since it actually preserves the ULA property, by Proposition IV.2.8 it suffices to prove that each $F_{n}(\mathcal{F})$ is perverse on the complement of all diagonals in $X^{n}$. This is a local question, so by Proposition IV.6.16 it suffices to show that $\left.F_{n}(\mathcal{F})\right|_{\vec{x}}[-n]$ is perverse for some (any) point $\vec{x} \in X^{n}$. Since the fiber of $\mathrm{Gr}_{G, X^{n}}$ is $\mathrm{Gr}_{G}^{n} \cong \mathrm{Gr}_{G^{n}}$ this is then a consequence of Proposition IV.7.2.

Finally, $F_{n}$ preserves convolution. As usual, Lemma IV.2.14 allows us to assume that we are applying it to the convolution of two ULA sheaves, since both $F_{n}$ and convolution are compatible with nearby cycles. Now we use the fact that

$$
\mathcal{F}_{1} *_{o} \mathcal{F}_{2}=\left.j_{! *}\left(\mathcal{F}_{1} \otimes \mathcal{F}_{2}\right)\right|_{U}
$$

from Proposition IV.3.4 (1), where both sides are ULA. Obviously,

$$
\left.\left.F_{n+m}\right|_{U}\left(\mathcal{F}_{1} \otimes \mathcal{F}_{2}\right)\right|_{U}=\left.\left(F_{n}\left(\mathcal{F}_{1}\right) \otimes F_{m}\left(\mathcal{F}_{2}\right)\right)\right|_{U}
$$

and since $F_{k}$ preserves the ULA property, we have

$$
\begin{aligned}
F_{n+m}\left(\mathcal{F}_{1} *_{o} \mathcal{F}_{2}\right) & =j_{!_{*}}\left(\left.F_{n+m}\left(\mathcal{F}_{1} *_{o} \mathcal{F}_{2}\right)\right|_{U}\right) \\
& =\left.j_{! *}\left(F_{n}\left(\mathcal{F}_{1}\right) \otimes F_{m}\left(\mathcal{F}_{2}\right)\right)\right|_{U}=F_{n}\left(\mathcal{F}_{1}\right) *_{o} F_{m}\left(\mathcal{F}_{2}\right) .
\end{aligned}
$$

This is what we want.

The proof would likewise show that $F_{n}$ preserves the commutativity constraint of $*_{i}$ except that the shift operator in $\mathscr{D}$, which appears in its definition, is not a tensor functor but rather a graded tensor functor, where the grading is $(-1)^{i j}$ for the product of complexes in degrees $i$ and $j$. In particular, as defined, the commutativity constraints on $\mathrm{Gr}_{G, X^{n}}$ and on $\mathrm{Gr}_{T, X^{n}}$ differ by a factor of

$$
(-1)^{\langle 2 \rho, \lambda\rangle\langle 2 \rho, \mu\rangle}
$$

for the convolution of weight spaces supported on $\mathrm{Gr}_{T, X^{n}}^{\lambda}$ and $\operatorname{Gr}_{T, X^{n}}^{\mu}$. Note that the function $\operatorname{Gr}_{G, X^{n}}^{\lambda} \mapsto\langle 2 \rho, \lambda\rangle(\bmod 2)$ is constant on connected components of $\mathrm{Gr}_{G, X^{n}}$ by Proposition IV.1.1, so we may modify the natural commutativity constraint on $*_{i}$ by the above factor, when two sheaves supported on the appropriate components are convolved. Thus, we conclude:

Corollary IV.4.3. From this point on, let $\mathbf{S p h}{ }^{U L A}\left(\mathscr{G}_{n}\right)$ denote the same category as usual but whose tensor structure has its commutativity constraint modified as above. Then the fiber functor

$$
F_{n}: \mathbf{S p h}^{U L A}\left(\mathscr{G}_{n}\right) \rightarrow \mathbf{S p h}^{U L A}\left(\mathscr{T}_{n}\right)
$$

is a tensor functor. 
Parabolic fiber functors. We will need some generalizations of $F_{n}$, described in [BD, 5.3.27-31]. Let $P$ be a parabolic subgroup of $G$ with unipotent radical $M$ and Levi quotient $L=P / M$, and recall the diagram (II.15)

$$
\mathrm{Gr}_{G, X^{n}} \stackrel{p}{\leftarrow} \mathrm{Gr}_{P, X^{n}} \stackrel{l}{\rightarrow} \mathrm{Gr}_{L, X^{n}} .
$$

First of all, it is easily verified that

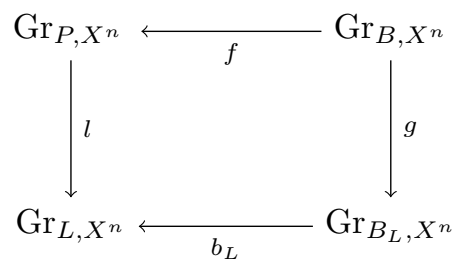

is a pullback diagram, where $B_{L}=B / N$ is a Borel subgroup in $L, b_{L}$ is the $b$ corresponding to the reductive group $L, f$ is the map corresponding to the inclusion $B \rightarrow P$, and $g$ to the projection $B \rightarrow B_{L}$. Thus, the components $\operatorname{Gr}_{B, X^{n}}^{\lambda}$ sent by $f$ into the same component of $\operatorname{Gr}_{P, X^{n}}$ are indexed by those $\lambda$ for which $b_{L}$ sends $\mathrm{Gr}_{B_{L}, X^{n}}$ to the same component of $\mathrm{Gr}_{L, X^{n}}$; namely, they are indexed by $\Lambda / \Lambda_{L}$, where $\Lambda_{L}$ is the coroot lattice of $L$. Thus, we can make the following definition.

Definition IV.4.4. With notation as above, also let $2 \rho_{L}$ be the sum of the positive roots in $L$ (with respect to $B_{L}$ ), and $2 \rho_{G / L}=2 \rho-2 \rho_{L}$. For $\mathcal{F} \in \mathbf{S p h}\left(\mathscr{G}_{n}\right)$, let $p^{*} \mathcal{F}=\bigoplus_{\Lambda / \Lambda_{L}} \mathcal{F}^{P, \lambda}$ with the summands supported on the $\lambda$-part of $\operatorname{Gr}_{P, X^{n}}$. Then the $\lambda$-weight space of the parabolic fiber functor $F_{n}^{P}$ is

$$
\left(F_{n}^{P}\right)_{!}^{*}(\mathcal{F})_{\lambda}=l_{!} \mathcal{F}^{P, \lambda}\left[\left\langle 2 \rho_{G / L}, \lambda\right\rangle\right]
$$

where the shift does not depend on the representative of $\lambda$ in $\Lambda$. Likewise, we define $\left(F_{n}^{P}\right)_{*}^{!}$.

\section{Proposition IV.4.5.}

(1) $\left(F_{n}\right)_{!}^{*} \circ\left(F_{n}^{P}\right)_{!}^{*}=\left(F_{n}\right)_{!}^{*}$ and $\left(F_{n}\right)_{*}^{!} \circ\left(F_{n}^{P}\right)_{*}^{!}=\left(F_{n}\right)_{*}^{!}$, where the left-hand $F_{n}$ refers to that defined for the reductive group $L$. In particular, $\left(F_{n}^{P}\right)_{!}^{*}=$ $\left(F_{n}^{P}\right)_{*}$.

(2) Let $P_{1} \subset P_{2}$ be two parabolic subgroups of $G$, with corresponding fiber functors $F_{n}^{P_{1}}, F_{n}^{P_{2}}$, and let $P_{L}=P_{1} \cap L_{2}$; then $F_{n}^{P_{1}}=F_{n}^{P_{L}} \circ F_{n}^{P_{2}}$.

(3) $F_{n}^{P}$ is t-exact, faithful, and respects convolution and the ULA property.

Proof. For (1), just use the diagram (IV.6) and the fact that $b_{L}^{*} l_{!}=g_{!} f^{*}$ and likewise for $*$ and ! reversed to get the first claim. Since $F_{n}$ is faithful and sends the natural map (defined in Bra03] $)\left(F_{n}^{P}\right)_{!}^{*} \rightarrow\left(F_{n}^{P}\right)_{!}^{*}$ to an isomorphism, that map is an isomorphism; likewise, it preserves the isomorphism property of the natural map in Definition IV.2.6. Item (2) is proven using the evident two-parabolics version of (IV.6) in the same way. For (3) the first two claims follow from $F_{n} \circ F_{n}^{P}=F_{n}$ and the fact that $F_{n}$ is $t$-exact and faithful. Since $p$ is proper, the last two are proven exactly as in Proposition IV.4.2

It is easily verified that the grading of the commutativity constraint which makes $F_{n}$ a tensor functor also makes each $F_{n}^{P}$ a tensor functor. 


\section{IV.5. THE MAIN THEOREM FOR A TORUS}

We combine the constructions already given to establish the main theorem in the special case when $G=T$ is a torus; as usual, we let $\mathscr{T}_{n}$ be the sf $k^{*}$-gerbe on $\operatorname{Gr}_{T, X^{n}}$. First, we complete the correspondence begun in Proposition II.4.9 by describing it for $\operatorname{Sph}\left(\mathscr{T}_{n}\right)$.

Proposition IV.5.1. There exists an object $\mathcal{F} \in \mathbf{S p h}^{U L A}\left(\mathscr{T}_{n}\right)$ living on $\mathrm{Gr}_{T, X}^{\lambda_{1}, \ldots, \lambda_{n}}$ only if we have $\kappa\left(\lambda_{i}, \mu\right)=1$ for all $\mu \in \Lambda_{T}$, where $\kappa$ is the bilinear form defined by $\mathscr{T}_{n}$.

Proof. If such a twisted sheaf exists, then by Lemma 1.3.12, $\mathscr{T}_{n}^{\lambda_{1}, \ldots, \lambda_{n}}$ is equivariantly trivial. By the construction of Theorem III.2.10, we have $\kappa\left(\lambda_{i}, \mu\right)=1$ for all $\mu$.

Let $Q$ be the quadratic form associated with $\mathscr{T}_{n}$ and denote by $\Lambda_{Q}$ the kernel of its associated bilinear form $\kappa$; we define $\check{T}_{Q}$ to be the torus whose weight lattice is $\Lambda_{Q}$ and ${ }^{L} \check{T}_{Q}$ its dual. Then by the above we may consider $\operatorname{Sph}\left(\mathscr{T}_{n}\right)$ to consist of sheaves on $\operatorname{Gr}_{L} \breve{T}_{Q}, X^{n}$, where $\mathscr{T}_{n}$ is equivariantly trivial and so its spherical twisted sheaves coincide simply with $\operatorname{Perv}\left(\mathscr{T}_{n}\right)$. By Proposition II.4.3, $\mathscr{T}_{n}$ is a commutative multiplicative sf $k^{*}$-gerbe on this restricted grassmannian, and so by Proposition II.4.9 corresponds to some sf comultiplicative gerbe $\mathscr{Z}_{n}$ for the sheaf of groups $\operatorname{Fact}\left(\tilde{T}_{Q}(k)\right)_{n}$ (previously called $\check{T}_{Q}\left(k^{*}\right)$ by abuse of notation, but the definitions are the same).

Proposition IV.5.2. We have $\operatorname{Sph}\left(\mathscr{T}_{n}\right) \cong \mathscr{Z}_{n} \otimes \mathbf{F R e p}_{n}\left(\check{T}_{Q}\right)$, the equivalence pairing $\mathbf{S p h}^{U L A}\left(\mathscr{T}_{n}\right)$ with lisse sheaves.

Proof. We will write $p_{n}: \operatorname{Gr}_{T, X^{n}} \rightarrow X^{n}$ for the structure map of the grassmannian. To construct an equivalence $\operatorname{Sph}\left(\mathscr{T}_{n}\right) \rightarrow \mathscr{Z}_{n} \otimes \mathbf{F R e p}_{n}\left(\check{T}_{Q}\right)$, it suffices by Lemma IV.2.14 to construct a horizontal functor

$$
\operatorname{Sph}^{\mathrm{ULA}}\left(\mathscr{T}_{n}\right) \rightarrow \mathscr{Z}_{n} \otimes \mathbf{F R e p}_{n}^{\mathrm{ULA}}\left(\check{T}_{Q}\right),
$$

which is an equivalence. Let $\mathcal{F} \in \operatorname{Sph}\left(\mathscr{T}_{n}\right)$, so that the topological structure of $\mathrm{Gr}_{T, X^{n}}$ given in Proposition II.1.2 allows us to write, for any partition $p$ of $n$ with $m$ parts, a direct sum decomposition

$$
\left.\mathcal{F}\right|_{X_{p}^{n}} \cong \bigoplus_{\lambda_{i} \in X^{*}\left(\check{T}_{Q}\right)} \mathcal{F}_{p}^{\lambda_{1}, \ldots, \lambda_{m}}
$$

such that if $p^{\prime}$ refines $p$, then its corresponding decomposition refines that of $p$ over $X_{p^{\prime}}^{n}$. However, if $\mathcal{F}$ is ULA, then by Proposition IV.2.8 it is functorially determined by its restriction to the complement of all diagonals in $X^{n}$, and thus we can simply write

$$
\mathcal{F} \cong \bigoplus \mathcal{F}^{\lambda_{1}, \ldots, \lambda_{n}}
$$

with each component sheaf supported on $\operatorname{Gr}_{T, X^{n}}^{\lambda_{1}, \ldots, \lambda_{n}}$. This gives $\mathcal{F}$ an action of $\left(\check{T}_{Q}\right)^{n}$ via a grading by its weight lattice. Furthermore, since $\check{T}_{Q}^{n}$ acts on $\mathcal{F}^{\lambda_{1}, \ldots, \lambda_{n}}$ through the character $\left(\lambda_{1}, \ldots, \lambda_{n}\right)$, the $\mathscr{T}_{n}$-twisting on $\mathcal{F}$ becomes a $\mathscr{Z}_{n}$-twisting according to the construction given in Proposition II.4.9, and so we have the desired functor, equal to $\left(p_{n}\right)_{*}$ :

$$
\operatorname{Sph}^{\mathrm{ULA}}\left(\mathscr{T}_{n}\right) \rightarrow \mathscr{Z}_{n} \otimes \operatorname{Rep}^{\mathrm{ULA}}\left(\check{T}_{Q}^{n}\right)=\mathscr{Z}_{n} \otimes \mathbf{F R e p}_{n}^{\mathrm{ULA}}\left(\check{T}_{Q}\right) .
$$


By definition, it is an equivalence, and by Lemma IV.2.12 it is horizontal because $p_{n}$ is ind-finite (so ind-proper), so we are done.

To finish the picture, we must show how convolution is carried by this equivalence. First, we have a comparison of convolution on $\mathrm{Gr}_{T, X^{n}}$ with the ordinary tensor product. We recall that since objects of $\mathbf{S p h}\left(\mathscr{T}_{n}\right)$ are supported on $\operatorname{Gr}_{L} \breve{T}_{Q}, X^{n}$, where $\mathscr{T}_{n}$ is multiplicative factorizable, we have natural equivalences

$$
\mathscr{T}_{n}^{\lambda_{1}, \ldots, \lambda_{n}} \otimes \mathscr{T}_{m}^{\mu_{1}, \ldots, \mu_{m}} \cong \mathscr{T}_{n+m}^{\lambda_{1}, \ldots, \lambda_{n}, \mu_{1}, \ldots, \mu_{m}}
$$

so that the twisted outer tensor product on components of $\mathrm{Gr}_{L} \check{T}_{Q}, X^{n}$ and $\operatorname{Gr}_{L} \breve{T}_{Q}, X^{m}$ :

$$
\operatorname{Perv}\left(\mathscr{T}_{n}^{\lambda_{1}, \ldots, \lambda_{n}}\right) \times \operatorname{Perv}\left(\mathscr{T}_{m}^{\mu_{1}, \ldots, \mu_{m}}\right) \rightarrow \operatorname{Perv}\left(\mathscr{T}_{n}^{\lambda_{1}, \ldots, \lambda_{n}} \otimes \mathscr{T}_{m}^{\mu_{1}, \ldots, \mu_{m}}\right)
$$

takes its values in $\operatorname{Perv}\left(\mathscr{T}_{n+m}^{\lambda_{1}, \ldots, \lambda_{n}, \mu_{1}, \ldots, \mu_{m}}\right)$. We will refer to this as simply the outer tensor product. There is also a natural inner tensor product of ULA sheaves obtained when $n=m$ by restricting to the diagonal copy of $X^{n}$.

Proposition IV.5.3. Outer and inner convolutions coincide with outer and inner tensor products.

Proof. As always, we work on $\mathrm{Gr}_{L_{T_{Q}}, X^{n}}$. Then, by Lemma III.1.14, the convolution diagrams are identified with ordinary products and the multiplication maps become identities on each component; since the $G(\widehat{\mathcal{O}})$-equivariance structures on both gerbe and sheaves are trivial, the twisted products become ordinary outer tensor products. This establishes the first claim, and the second follows by definition of inner convolution.

There is also a tensor product operation in $\mathscr{Z}_{n} \otimes \mathbf{F R e p}\left(\check{T}_{Q}\right)$.

Definition IV.5.4. Let $H$ be a group. The outer tensor product on $\operatorname{FRep}_{n}(H)$ and $\mathbf{F R e p}_{m}(H)$ is the composition of the outer tensor product of perverse sheaves,

$$
\operatorname{FRep}_{n}(H) \times \operatorname{FRep}_{m}(H) \rightarrow\left(\operatorname{Fact}(H)_{n} \otimes \operatorname{Fact}(H)_{m}\right)-\mathbf{M o d}
$$

(in perverse sheaves on $X^{n+m}$ ) with the forgetful map along the diagonal (II.10)

$$
\psi_{m, n}: \operatorname{Fact}(H)_{n+m} \hookrightarrow \operatorname{Fact}(H)_{n} \otimes \operatorname{Fact}(H)_{m}
$$

into $\operatorname{FRep}_{n+m}(H)$. The outer tensor product of twisted factorizable representations is

$$
\begin{aligned}
\left(\mathscr{Z}_{n} \otimes \mathbf{F R e p}_{n}(H)\right) \times\left(\mathscr{Z}_{m} \otimes \mathbf{F R e p}_{m}(H)\right) & \\
& \rightarrow\left(\mathscr{Z}_{n} \otimes \mathscr{Z}_{m}\right) \otimes\left(\operatorname{Fact}(H)_{n} \otimes \operatorname{Fact}(H)_{m}\right)-\operatorname{Mod} \\
\cong & { }^{2} \psi_{m, n}\left(\mathscr{Z}_{n+m}\right) \otimes\left(\operatorname{Fact}(H)_{n} \otimes \operatorname{Fact}(H)_{m}\right)-\operatorname{Mod} \\
& \rightarrow \mathscr{Z}_{n+m} \otimes \mathbf{F R e p}_{n+m}(H)
\end{aligned}
$$

(compare with (II.12) $)$. Likewise, we have an inner tensor product of twisted lisse sheaves obtained by restriction to the diagonal copy of $X^{n}$ in $X^{n} \times X^{n}$.

Then we can strengthen Proposition IV.5.2

Proposition IV.5.5. The equivalence of Proposition IV.5.2 is a tensor functor.

Proof. By Lemma IV.2.14, it is enough to prove this for ULA sheaves. But it is clear that $\left(p_{n}\right)_{*}$ sends one notion of tensor product to the other over the open subset of $X^{n}$ away from the diagonals, so by Proposition IV.2.8 it preserves the tensor product on all of $X^{n}$. 


\section{IV.6. Absolute twisted SAtake: Semisimplicity}

In this section we apply the preceding constructions to the category of twisted perverse sheaves on the absolute grassmannian $\mathrm{Gr}_{G}$.

Convolution on the absolute grassmannian. As is usual, we will compare this category to perverse sheaves on $\operatorname{Gr}_{G, X}$ for sufficiently small $X$. Thus, let $x \in X$ be a fixed point and replace $X$ by a small (contractible) disk neighborhood of $x$. We identify $\mathrm{Gr}_{G}=\left.\mathrm{Gr}_{G, X}\right|_{x}$; then we actually have an identification $\operatorname{Gr}_{G, X} \cong \mathrm{Gr}_{G} \times X$, since the coordinate on $X$ identifies all the fibers $\left.\operatorname{Gr}_{G, X}\right|_{y} \cong G\left(\widehat{\mathcal{K}}_{y}\right) / G\left(\widehat{\mathcal{O}}_{y}\right)$ with the one at $x$. We set $\mathscr{G}=\left.\mathscr{G}_{1}\right|_{x}$; it inherits a $G(\widehat{\mathcal{O}})=\left.G(\widehat{\mathcal{O}})_{X}\right|_{x}$-equivariance structure, and so we can define $\operatorname{Sph}(\mathscr{G})$, the category of $G(\widehat{\mathcal{O}})$-equivariant $\mathscr{G}$-twisted perverse sheaves on $\mathrm{Gr}_{G}$.

Definition IV.6.1. For $\mathcal{F}_{1}, \mathcal{F}_{2} \in \operatorname{Sph}(\mathscr{G})$, let $\mathcal{F}_{1} \widetilde{\otimes} \mathcal{F}_{2}$ be their twisted product on $\operatorname{Conv}_{G}$, defined as in Definition III.1.9 to be $G(\widehat{\mathcal{K}}) \times{ }^{G(\widehat{\mathcal{O}})} \operatorname{Gr}_{G}=\left.\operatorname{Conv}_{1}^{2}\right|_{x}$, and set

$$
\mathcal{F}_{1} * \mathcal{F}_{2}=m_{*}\left(\mathcal{F}_{1} \widetilde{\otimes} \mathcal{F}_{2}\right),
$$

relative to the proper map $m: \operatorname{Conv}_{G} \rightarrow \operatorname{Gr}_{G}$.

From the generalities above, it is easy to deduce the good properties of this convolution. To see this, let $\operatorname{Spr}: \mathbf{S p h}(\mathscr{G}) \rightarrow \mathbf{S p h}^{\mathrm{ULA}}\left(\mathscr{G}_{1}\right)$ be the "spreading" functor defined by

$$
\operatorname{Spr}(\mathcal{F})=\operatorname{pr}_{\mathrm{Gr}_{G}}^{*} \mathcal{F}[1],
$$

using $\mathrm{Gr}_{G, X} \cong \mathrm{Gr}_{G} \times X$. Of course, this depends on the decomposition but this will not matter. Clearly, Spr is left-inverted by the functor Res: $\mathbf{S p h}^{\mathrm{ULA}}\left(\mathscr{G}_{1}\right) \rightarrow$ $\operatorname{Sph}(\mathscr{G})$ defined by

$$
\operatorname{Res}(\mathcal{F})=\left.\mathcal{F}\right|_{x}[-1]
$$

Proposition IV.6.2. Let $\mathcal{F}_{1}, \mathcal{F}_{2} \in \operatorname{Sph}(\mathscr{G})$; then we have $\operatorname{Spr}\left(\mathcal{F}_{1}\right) *_{i} \operatorname{Spr}\left(\mathcal{F}_{2}\right)=$ $\operatorname{Spr}\left(\mathcal{F}_{1} * \mathcal{F}_{2}\right)$. In particular, $\mathcal{F}_{1} * \mathcal{F}_{2}$ is perverse and defines a rigid tensor structure on $\mathbf{S p h}(\mathscr{G})$.

Proof. The product $\operatorname{Gr}_{G, X} \cong \operatorname{Gr}_{G} \times X$ induces an identification $\operatorname{Conv}_{1}^{2} \cong \operatorname{Conv}_{G} \times X$ compatible with the multiplication maps. The first statement then follows from the definitions of the convolutions and proper base change. The second statement then follows by applying Res to Proposition IV.3.4 and Proposition IV.3.5.

Simple objects. We use this structure to show that $\mathbf{S p h}(\mathscr{G})$ is in fact a semisimple abelian category. First, we identify the irreducible objects: let $\mathcal{J}$ be any simple object of $\operatorname{Sph}(\mathscr{G})$; by general principles, it must be of the form $j_{!_{*}}(\mathcal{L})$, where $\mathcal{L}$ is a locally constant sheaf of $k$-vector spaces supported on a locally closed subset $j: S \rightarrow \operatorname{Gr}_{G}$; since $\mathcal{J}$ is $G(\widehat{\mathcal{O}})$-equivariant, $S$ must be a union of orbits, and so by Proposition IV.1.1 must have some $\operatorname{Gr}_{G}^{\lambda}$ as a dense open subset; we assume therefore that $S=\mathrm{Gr}_{G}^{\lambda}$. By Lemma IV.1.3, Lemma I.3.12 applies and so $\mathscr{G} \mid \mathrm{Gr}_{G}^{\lambda}$ and $\mathcal{L}$ are equivariantly trivial. We conclude:

Proposition IV.6.3. The simple objects of $\mathbf{S p h}(\mathscr{G})$ are all minimal extensions $\mathcal{J}(\lambda)$ from some $\operatorname{Gr}_{G}^{\lambda}$ of the constant sheaves $\underline{k}\left[\operatorname{dim} \mathrm{Gr}_{G}^{\lambda}\right]$.

We can say more about which coweights $\lambda$ occur. By Proposition IV.5.1 applied to $\operatorname{Spr}(\mathcal{J}(\lambda))$, we have $F_{1}(\operatorname{Spr}(\mathcal{J}(\lambda)))^{\lambda}=0$ if $\lambda \notin \Lambda_{Q}$, the kernel of the bilinear 
form $\kappa$ associated with $Q$. By Proposition IV.4.2](2), we have $\operatorname{Spr}(\mathcal{J}(\lambda))=0$, and therefore $\mathcal{J}(\lambda)=0$ as well. We arrive at the following more precise statement:

Proposition IV.6.4. We have $\mathcal{J}(\lambda)=0$ if $\lambda \notin \Lambda_{Q}$. Furthermore, for every coroot $\check{\alpha}$ of $G$ such that $Q(\check{\alpha})$ has infinite order in $A$, if for some $\lambda$ we have $\kappa(\check{\alpha}, \lambda)=1$, then $\langle\alpha, \lambda\rangle=0$.

Proof. The first statement was already argued. For the second statement, we need only apply Lemma II.7.1 with $\varepsilon_{\check{\alpha}}=1$ according to Theorem II.7.3.

$$
\kappa(\check{\alpha}, \lambda)=Q(\check{\alpha})^{\langle\alpha, \lambda\rangle} .
$$

If the base is not a root of unity, this is 1 if and only if the exponent is zero.

This leads to a finiteness result on the gerbe itself.

Corollary IV.6.5. Suppose $\lambda \in \Lambda_{Q}$; then $\mathscr{G}$ has finite order on $\overline{\mathrm{Gr}}_{G}^{\lambda}$.

Proof. We use Lemma II.7.7 that is, we stratify $\overline{\mathrm{Gr}}_{G}^{\lambda}$ by the intersections with the $\operatorname{Gr}_{B}^{\mu}$ such that $w_{0}(\lambda) \leq \mu \leq \lambda$, on each of which $\mathscr{G}$ is trivialized with the order of trivialization being $Q(\check{\alpha})^{-1}$ around each boundary divisor $\operatorname{Gr}_{B}^{\mu-\check{\alpha}}$. By Proposition IV.6.4 if $Q(\check{\alpha})$ does not have finite order, then $\lambda$, being in $\Lambda_{Q}$, must be orthogonal to $\alpha$, and therefore, if $\operatorname{Gr}_{B}^{\mu} \cap \overline{\mathrm{Gr}}_{G}^{\lambda} \neq \varnothing$, we also have $\mu$ orthogonal to $\alpha$. Therefore, the numbers $Q(\check{\alpha})^{-1}$ which occur are all of finite order, so $\mathscr{G}^{n}$ is trivial on $\overline{\mathrm{Gr}}_{G}$ for some $n$, as desired.

We begin to analyze the basic properties of extensions of these simple objects.

Lemma IV.6.6. We have $\operatorname{Ext}^{1}(\mathcal{J}(\lambda), \mathcal{J}(\lambda))=0$; i.e., there are no nontrivial extensions of $\mathcal{J}(\lambda)$ by itself.

Proof. Denote by $j: \mathrm{Gr}_{G}^{\lambda} \rightarrow \overline{\mathrm{Gr}}_{G}^{\lambda}$ the inclusion map, $i$ the inclusion of the complement. The property

$$
{ }^{p} H^{0}\left(i^{*} j_{! *} \mathcal{F}\right)=0={ }^{p} H^{0}\left(i^{!} j_{! *} \mathcal{F}\right),
$$

uniquely characterizes the functor $j_{! *}$ and is preserved under extensions, so that we have

$$
\operatorname{Ext}^{1}\left(j_{! *} \mathcal{F}_{1}, j_{! *} \mathcal{F}_{2}\right) \cong \operatorname{Ext}^{1}\left(\mathcal{F}_{1}, \mathcal{F}_{2}\right) .
$$

When $\mathcal{F}_{1}=\mathcal{F}_{2}=\underline{k}\left[\operatorname{dim} \mathrm{Gr}_{G}^{\lambda}\right]$, the latter is zero since $\operatorname{Gr}_{G}^{\lambda}$ is simply connected.

We now turn to more general extensions. In identifying simple factors of a spherical sheaf, the following lemma is key:

Lemma IV.6.7. Let $\mathcal{F} \in \operatorname{Sph}(\mathscr{G})$ have composition factors $\mathcal{J}\left(\lambda_{i}\right)$ for various dominant coweights $\lambda_{i}$; then the orbits $\mathrm{Gr}_{G}^{\lambda_{i}}$ are exactly those such that (denoting by $j$ their inclusions into $\left.\operatorname{Gr}_{G}\right)$ we have ${ }^{p} H^{0}\left(j^{*} \mathcal{F}\right) \neq 0$. More precisely, $\mathcal{J}(\nu)$ occurs $n$ times as a composition factor of $\mathcal{F}$ if and only if for $i:\left\{t^{\nu}\right\} \rightarrow \operatorname{Gr}_{G}$, we have

$$
{ }^{p} H^{0}\left(i^{*} \mathcal{F}\left[-\operatorname{dim} \operatorname{Gr}_{G}^{\nu}\right]\right) \cong k^{n} .
$$

Proof. The first statement follows from (IV.7). For the second, we know that the sheaf ${ }^{p} H^{0}\left(j^{*} \mathcal{F}\right)$ is $G(\widehat{\mathcal{O}})$-equivariant and therefore constant on $\mathrm{Gr}_{G}^{\nu}$, so vanishes if and only if its stalk at $t^{\nu}$ does. The more refined count comes from the fact that $j^{*} \mathcal{J}(\nu) \cong k\left[\operatorname{dim} \mathrm{Gr}_{G}^{\nu}\right]$. 
In order to produce such a nonvanishing stalk, we will use the following fact about the top cohomology of a proper scheme.

Lemma IV.6.8. Let $p: P \rightarrow$ Spec $\mathbb{C}$ be the structure map of a connected proper scheme of dimension $d$ and let $j: U \rightarrow P$ be the inclusion of a smooth open set whose complement $i: Z \rightarrow P$ has codimension at least 2 . If $A^{\bullet} \in{ }^{p} \mathscr{D} \leqslant 0$ and if $j^{*} A^{\bullet} \cong \underline{k}[d]$, then ${ }^{p} H^{d} p_{*}\left(A^{\bullet}\right) \cong k$.

Note that the lemma concerns untwisted perverse sheaves, but that if we are given a trivial (if not trivialized) gerbe on Spec $\mathbb{C}$, the same statement holds of twisted sheaves as well, since in fact they are equivalent to untwisted ones. There is no twisted version of this lemma because in order to apply cohomology (that is, pushforward), the gerbe on $P$ must be the pullback of that on $\mathrm{Spec} \mathbb{C}$, which is always trivial.

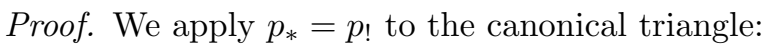

$$
j_{!} j^{*} A^{\bullet} \rightarrow A^{\bullet} \rightarrow i_{*} i^{*} A^{\bullet} \rightarrow \quad \Longrightarrow \quad\left(\left.p\right|_{U}\right) ! j^{*} A^{\bullet} \rightarrow p_{*} A^{\bullet} \rightarrow\left(\left.p\right|_{Z}\right)_{*} i^{*} A^{\bullet} \rightarrow .
$$

By hypothesis, $\operatorname{dim} Z \leq d-2$ and $i^{*} A^{\bullet} \in \in^{p} \mathscr{D} \leqslant 0$, so by [BBD82, $\left.\S 4.2 .4\right]$ and the long exact sequence of perverse cohomology we have, respectively,

$$
{ }^{p} H^{d}\left(\left.p\right|_{Z}\right)_{*} i^{*} A^{\bullet}={ }^{p} H^{d-1}\left(\left.p\right|_{Z}\right)_{*} i^{*} A^{\bullet}=0 \quad \Longrightarrow \quad{ }^{p} H^{d} p_{*}\left(A^{\bullet}\right) \cong{ }^{p} H^{d}\left(\left.p\right|_{U}\right)_{!}\left(j^{*} A^{\bullet}\right) .
$$

The last term is the dual of $H^{-d}\left(\left.p\right|_{U}\right)_{*}\left(j^{*} \mathbb{D} A^{\bullet}\right)$ on Spec $\mathbb{C}$, and we have

$$
H^{-d}\left(\left.p\right|_{U}\right)_{*}\left(j^{*} \mathbb{D} A^{\bullet}\right)=H^{-d}\left(\left.p\right|_{U}\right)_{*}(\mathbb{D} \underline{k}[d])=H^{0}\left(\left.p\right|_{U}\right)_{*} \underline{k}=\Gamma(U, \underline{k})=k,
$$

using $\mathbb{D}(\underline{k}[d])=\underline{k}[d]$ since $U$ is smooth.

Summands of a convolution. In particular, we may apply this to obtain composition factors of a convolution. For simplicity, we use the notation $l=\operatorname{dim} \operatorname{Gr}_{G}^{\lambda}$, $m=\operatorname{dim} \mathrm{Gr}_{G}^{\mu}, n=\operatorname{dim} \mathrm{Gr}_{G}^{\nu}$.

Lemma IV.6.9. For any dominant $\nu$, let $U=m^{-1}\left(t^{\nu}\right) \cap\left(\mathrm{Gr}_{G}^{\lambda} * \mathrm{Gr}_{G}^{\mu}\right)$. Then

$$
\operatorname{dim} U \leq \frac{1}{2}(l+m-n),
$$

with equality if and only if $\mathcal{J}(\nu)$ is a composition factor of $\mathcal{J}(\lambda) * \mathcal{J}(\mu)$. Furthermore, the multiplicity of this summand is the number of components of $U$ having this dimension.

Proof. Note that (IV.9) is equivalent, by Proposition IV.1.1 and Lemma IV.1.5, to

$$
\operatorname{codim} U-\operatorname{dim} U \geq n \text {. }
$$

For brevity, let $\mathcal{F}=\mathcal{J}(\lambda) \widetilde{\otimes} \mathcal{J}(\mu)$; it lives on the closure $C$ of $\operatorname{Gr}_{G}^{\lambda} * \mathrm{Gr}_{G}^{\mu}$ and is constant on this convolution product of orbits itself; we will denote

$$
P=\bar{U}=m^{-1}\left(t^{\nu}\right) \cap C .
$$

To evaluate $(\mathrm{IV} .8)$ for $m_{*}(\mathcal{F})$, we apply proper base change and then Lemma IV.6.8 with $A^{\bullet}=\left.\mathcal{F}\right|_{P}[-\operatorname{codim} P]$. Its restriction to $U$ is constant in $\operatorname{degree}-\operatorname{dim} P$ and the boundary of $U$ in $P$ has codimension 2 by Proposition IV.1.1 so the lemma applies and we conclude that the extremal case of [IV.10)] is the precise condition necessary for Lemma IV.6.7 to apply. If the left side were decreased, then Lemma IV.6.8 would produce positive-degree cohomology sheaves of $i^{*} m_{*}(\mathcal{F})[-n]$ and therefore of $m_{*}(\mathcal{F})$ and, finally, of $\mathcal{J}(\lambda) * \mathcal{J}(\mu)$, in contradiction to the fact that 
this is perverse. This gives the inequality of (IV.9) The statement on multiplicity follows from Lemma IV.6.7.

Corollary IV.6.10. The convolution $\mathcal{J}(\lambda) * \mathcal{J}(\mu)$ has exactly one copy of $\mathcal{J}(\lambda+\mu)$ as a composition factor, and all other ones $\mathcal{J}(\nu)$ have $\nu \leq \lambda+\mu$.

Proof. This follows directly from Lemma IV.6.9 and Proposition IV.1.6.

Finally, the last step on the way to general semisimplicity is to show that all the composition factors of a convolution are actually summands. This lemma, with the same proof, appeared as Gin95, Proposition 2.2.1] but was absent from MV07 since the latter paper proves semisimplicity of $\mathbf{S p h}$ by a different, more computational route.

Lemma IV.6.11. The convolution of two $\mathcal{J}(\lambda)$ 's is semisimple in $\mathbf{S p h}(\mathscr{G})$.

Proof. We have $\mathcal{J}(\lambda) * \mathcal{J}(\mu)=m_{*}(\mathcal{J}(\lambda) \widetilde{\otimes} \mathcal{J}(\mu))$, where the twisted product is on $\operatorname{Conv}_{G}$. It is the minimal extension from $\operatorname{Gr}_{G}^{\lambda} * \mathrm{Gr}_{G}^{\mu} \subset \operatorname{Conv}_{G}$ of the constant sheaf, and therefore simple. We would like to apply the decomposition theorem BBD82, Théorème 6.2.5], for which we must return to untwisted sheaves.

First, we obviously have nothing to do if $\mathcal{J}(\lambda)=0$ or $\mathcal{J}(\mu)=0$. If both are nonzero, then by Corollary IV.6.10. $\mathcal{J}(\lambda+\mu) \neq 0$ as well, and therefore $\mathscr{G}$ is trivial on $U=\mathrm{Gr}_{G}^{\lambda+\mu}$, since it possesses a twisted $k^{*}$-torsor there. By the same result, $\mathcal{J}(\lambda) * \mathcal{J}(\mu)$ is supported on its closure, and since $U$ is a dense open subspace of it, there is some $\mathscr{G}$-trivializing open cover $\left\{V_{i}\right\}$ of that closure all of whose elements intersect $U$, and we choose trivializations.

The trivializations on the $V_{i}$ entail trivializations of $m^{*}(\mathscr{G}) \cong \mathscr{G} \widetilde{\nabla} \mathscr{G}$ on the $m^{-1}\left(V_{i}\right)$, which differ by $k^{*}$-torsors on the intersections. Since all of them intersect $m^{-1}(U)$, they all intersect the set $\widetilde{U}=\mathrm{Gr}_{G}^{\lambda} * \mathrm{Gr}_{G}^{\mu}$ on which the twisted product $\mathcal{F}=\mathcal{J}(\lambda) \widetilde{\otimes} \mathcal{J}(\mu)$ is a constant sheaf, so it is a local system on each $m^{-1}\left(V_{i}\right) \cap \widetilde{U}$. Thus, on $m^{-1}\left(V_{i}\right)$, we have that $\mathcal{F}$ is the minimal extension of a local system, and thus is of geometric origin if that local system has finite monodromy. Indeed, by Corollary IV.6.5. $\mathscr{G}$ itself has finite order on $\overline{\mathrm{Gr}}_{G}^{\lambda+\mu}$ and, in particular, all the transition torsors of $m^{*}(\mathscr{G})$ are of finite order, and thus have finite monodromy as desired.

It follows that on each $m^{-1}\left(V_{i}\right)$, the twisted product $\mathcal{F}=\mathcal{J}(\lambda) \widetilde{\nabla} \mathcal{J}(\mu)$ is simple of geometric origin, so since $m$ is proper, the decomposition theorem does apply (this could have been done more directly using Kashiwara's conjecture, proved in Dri01]). Therefore, $\mathcal{J}(\lambda) * \mathcal{J}(\mu)$ is semisimple on each $V_{i}$. Its summands are isomorphic up to the product with local systems on the $V_{i} \cap V_{j}$, so the convolution itself is a direct sum of twisted sheaves, each of which is locally on the $V_{i}$ a direct sum of multiple copies of $\mathcal{J}(\nu)$ for one coweight $\nu$. But by Lemma IV.6.6, there are no extensions of $\mathcal{J}(\nu)$ by itself, so each of these summands is itself semisimple.

The preceding results allow us to prove the analogue for perverse sheaves of the following proposition, true for representations of any reductive group $H$.

Lemma. If $\lambda$ is a dominant weight in the root lattice of $H$, then there is some $\mu$ such that $V^{\lambda}$ is a direct summand of $\left(V^{\mu}\right)^{*} \otimes V^{\mu}$.

Lemma IV.6.12. Let $\lambda$ be a dominant coweight and a sum of simple coroots. Then there exists a $\mu$ such that $\mathcal{J}(\lambda)$ is a direct summand of $\mathcal{J}(\mu)^{*} * \mathcal{J}(\mu)$. 
Proof. Since the convolution is semisimple, this is equivalent to

$$
0 \neq \operatorname{Hom}\left(\mathcal{J}(\lambda), \mathcal{J}(\mu)^{*} * \mathcal{J}(\mu)\right)=\operatorname{Hom}(\mathcal{J}(\mu) * \mathcal{J}(\lambda), \mathcal{J}(\mu))
$$

and therefore to finding a copy of $\mathcal{J}(\mu)$ as a summand of $\mathcal{J}(\mu) * \mathcal{J}(\lambda)$, for some $\mu$. By the criterion of Lemma IV.6.9 we need only find an irreducible component of the fiber of $\mathrm{Gr}_{G}^{\mu} * \mathrm{Gr}_{G}^{\lambda}$ over $t^{\mu}$ with dimension at least (hence equal to) $l / 2$. This was already provided in Proposition IV.1.7.

Semisimplicity and consequences. We give a slick demonstration of the semisimplicity of Sph by an argument of Frenkel, Gaitsgory, and Vilonen from [FGV01, $\S 6.1]$.

Proposition IV.6.13. $\operatorname{Sph}(\mathscr{G})$ is semisimple.

Proof. It suffices to show that there are no nontrivial extensions of the irreducible objects, so we must show that $\operatorname{Ext}^{1}(\mathcal{J}(\lambda), \mathcal{J}(\mu))=0$ always. Since $\operatorname{Ext}^{1}$ is a derived functor of Hom, by the properties of the dual we have

$$
\operatorname{Ext}^{1}(\mathcal{J}(\lambda), \mathcal{J}(\mu))=\operatorname{Ext}^{1}\left(1, \mathcal{J}(\lambda)^{*} * \mathcal{J}(\mu)\right) .
$$

By Lemma IV.6.11, the latter sheaf is semisimple, so we may assume it is just of the form $\mathcal{J}(\lambda)$. If $\lambda$ is not in the coroot lattice, then $\mathcal{J}(\lambda)$ and 1 are supported on different connected components of $\mathrm{Gr}_{G}$, so of course have no nontrivial extensions. Otherwise, Lemma IV.6.12 applies and it suffices to replace the right-hand side with $\mathcal{J}(\mu)^{*} * \mathcal{J}(\mu)$. Then

$$
\operatorname{Ext}^{1}\left(1, \mathcal{J}(\mu)^{*} * \mathcal{J}(\mu)\right)=\operatorname{Ext}^{1}(\mathcal{J}(\mu), \mathcal{J}(\mu))=0,
$$

by Lemma IV.6.6.

Corollary IV.6.14. Let $\lambda \in \Lambda_{T}$ be dominant and denote by $j: \mathrm{Gr}_{G}^{\lambda} \rightarrow \overline{\mathrm{Gr}}_{G}^{\lambda}$ the open inclusion, and let $l=\operatorname{dim} \operatorname{Gr}_{G}^{\lambda}$. Then the natural maps are isomorphisms:

$$
\mathcal{J}_{!}(\lambda)=j_{!}(\underline{k}[l]) \rightarrow \mathcal{J}(\lambda)=j_{! *}(\underline{k}[l]) \rightarrow \mathcal{J}_{*}(\lambda)=j_{*}(\underline{k}[l]) .
$$

Proof. The complex $\mathcal{J}_{!}(\lambda)$ is a priori perverse in nonpositive degrees. Starting from the natural map, we get a distinguished triangle

$$
A^{\bullet} \rightarrow \mathcal{J}_{!}(\lambda) \rightarrow \mathcal{J}(\lambda) \rightarrow
$$

and since $\mathcal{J}(\lambda)$ is perverse, the long exact sequence of cohomology gives a short exact sequence in degree zero:

$$
0 \rightarrow{ }^{p} H^{0}\left(A^{\bullet}\right) \rightarrow{ }^{p} H^{0}\left(\mathcal{J}_{!}(\lambda)\right) \rightarrow \mathcal{J}(\lambda) .
$$

The last map is surjective by definition, as well as $G(\widehat{\mathcal{O}})$-equivariant by functoriality, so the first term is in $\mathbf{S p h}(\mathscr{G})$ and thus, by semisimplicity, the exact sequence splits. Applying $j^{*}$, we find that ${ }^{p} H^{0}\left(A^{\bullet}\right)$ is in the image of $i_{*}$, but after applying $i^{*}$ we find, since $i^{*} j_{!}=0$ and $i^{*}$ is right $t$-exact, that it vanishes (we use the splitting here). Thus, $\mathcal{J}(\lambda) \cong{ }^{p} H^{0}\left(\mathcal{J}_{!}(\lambda)\right)$.

Going back to the distinguished triangle, application of $j^{*}$ shows that $A^{\bullet}$ is in the image of $i_{*}$, and application of $i^{*}$ gives that $A^{\bullet} \cong i_{*} i^{*} \mathcal{J}(\lambda)[-1]$, so we have

$$
A^{\bullet} \cong i_{*} i^{* p} H^{0}\left(\mathcal{J}_{!}(\lambda)\right)[-1] \cong i_{*}{ }^{p} H^{0}\left(i^{*} \mathcal{J}_{!}(\lambda)\right)[-1]=0 .
$$

Therefore, $\mathcal{J}(\lambda) \cong \mathcal{J}_{!}(\lambda)$, as desired. The other isomorphism follows from duality.

In the coda, we compare $\operatorname{Sph}(\mathscr{G})$ to $\operatorname{Sph}^{\mathrm{ULA}}\left(\mathscr{G}_{1}\right)$. 
Lemma IV.6.15. The simple objects of $\mathbf{S p h}{ }^{U L A}\left(\mathscr{G}_{1}\right)$ are minimal extensions from some $\operatorname{Gr}_{G, X}^{\lambda}$ of locally constant sheaves $\mathcal{L}\left[\operatorname{dim} \mathrm{Gr}_{G, X}^{\lambda}\right]$ which are trivial on the fibers over $X$.

Proof. The same argument as for $\mathrm{Gr}_{G}$ shows that they are all of the form $j_{!_{*}}(\mathcal{F})$, where $j$ is the inclusion of one of these orbits and $\mathcal{F} \in \operatorname{Sph}^{\mathrm{ULA}}\left(\mathscr{G}_{1}\right)$ is a simple sheaf on $\operatorname{Gr}_{G}^{\lambda}$. Since $\mathcal{F} \mid \mathrm{Gr}_{T, X}^{\lambda}\left[-\operatorname{dim}_{X} \mathrm{Gr}_{G, X}^{\lambda}\right]$ is again ULA, it must be lisse on $\mathrm{Gr}_{T, X}^{\lambda} \cong X$ by Corollary IV.2.9. Considering $\operatorname{Gr}_{T, X}^{\lambda} \cong X$, by $G(\widehat{\mathcal{O}})_{X}$-equivariance $\mathcal{F} \mid \mathrm{Gr}_{G, X}^{\lambda}$ is the pullback from it of a lisse sheaf, hence lisse, and so Lemma I.3.12 applies as in Proposition IV.6.3.

In particular, when $X$ is cohomologically trivial, we must have $\mathcal{L}=\underline{k}$; let $\mathcal{J}(\lambda)_{X}$ be the corresponding irreducible objects. Lemma IV.6.11 holds for these objects with the same proof, so the semisimple abelian category generated by these objects is closed under $*_{i}$. The obvious equivalence (by Proposition IV.6.13) with $\mathbf{S p h}(\mathscr{G})$ which identifies their irreducible objects is by definition an equivalence of tensor categories that does not depend on the choice of isomorphism $\mathrm{Gr}_{G, X} \cong \mathrm{Gr}_{G} \times X$. In particular, the tensor structure (in particular, the commutativity constraint) on $\operatorname{Sph}(\mathscr{G})$ is independent of this isomorphism, of the choice of $x \in X$, and of the neighborhood containing $x$ on which the isomorphism was given.

Finally, we can completely describe the ULA sheaves in a neighborhood of a point.

Proposition IV.6.16. Let $X$ be a small (contractible) neighborhood of one of its points; then we have a natural equivalence $\mathbf{S p h}^{U L A}\left(\mathscr{G}_{1}\right) \cong \mathbf{S p h}(\mathscr{G})$ of tensor abelian categories. Likewise, in any small neighborhood in $X^{n}$ not intersecting any diagonal, we have $\mathbf{S p h}^{U L A}\left(\mathscr{G}_{n}\right) \cong \mathbf{S p h}\left(\mathscr{G}^{\otimes n}\right)$.

Proof. First, we observe that $\mathbf{S p h}{ }^{\mathrm{ULA}}\left(\mathscr{G}_{1}\right)$ is semisimple. To accomplish this, the same proof of Proposition IV.6.13 will work if we can establish Lemma IV.6.12 for the $\mathcal{J}(\lambda)_{X}$. But the existence of a summand such as the lemma claims can be detected after application of Res by semisimplicity of inner convolution, so the lemma reduces to that on $\mathrm{Gr}_{G}$, which is already proven.

Therefore both $\mathbf{S p h}^{\mathrm{ULA}}\left(\mathscr{G}_{1}\right)$ and $\operatorname{Sph}(\mathscr{G})$ are semisimple tensor abelian categories whose simple objects are identified in such a way that their convolutions agree. Thus, the equivalence which sends one set of irreducibles to the other is a tensor equivalence.

For the last statement we simply observe that on such a neighborhood, $\operatorname{Gr}_{G, X^{n}} \cong$ $X^{n} \times \mathrm{Gr}_{G}^{n}$, with $X^{n}$ contractible and $\mathrm{Gr}_{G}^{n} \cong \mathrm{Gr}_{G^{n}}$, so that the same proof applies.

\section{IV.7. Absolute twisted Satake: Root data}

We turn to the twisted Satake equivalence on $\mathrm{Gr}_{G}$. The results of this section generalize and simplify those of [FL10].

The absolute fiber functor. First, we define the fiber functor for $\mathbf{S p h}(\mathscr{G})$. As for $\operatorname{Sph}\left(\mathscr{G}_{n}\right)$, we have the fundamental diagram

$$
\mathrm{Gr}_{G} \stackrel{b}{\leftarrow} \mathrm{Gr}_{B} \stackrel{t}{\rightarrow} \mathrm{Gr}_{T}
$$


and the sf $k^{*}$-gerbe $\mathscr{T}$ on $\operatorname{Gr}_{T}$ such that $t^{*} \mathscr{T} \cong b^{*} \mathscr{G}$; in the notation of the previous section, we also have $\mathscr{T}=\left.\mathscr{T}_{1}\right|_{x}$. It is noncanonically trivial as a gerbe, but not as a multiplicative gerbe, so we continue to use it in the notation.

Definition IV.7.1. The fiber functor $F: \operatorname{Sph}(\mathscr{G}) \rightarrow \mathscr{D}(\mathscr{T})$ is the following functor: for $\mathcal{F} \in \mathbf{S p h}(\mathscr{G})$, let $\mathcal{F}^{\lambda}$ be the restriction of $b^{*} \mathcal{F}$ to the connected component $\mathrm{Gr}_{B}^{\lambda}$, and set

$$
F(\mathcal{F})^{\lambda}=F_{!}^{*}(\mathcal{F})^{\lambda}=t_{!}^{\lambda} \mathcal{F}^{\lambda}[\langle 2 \rho, \lambda\rangle]
$$

to be the component of $F(\mathcal{F})$ on $\operatorname{Gr}_{T}^{\lambda}$.

Equivalently, it is clear that (again in the previous notation) we have

$$
\operatorname{Res} F_{1}(\operatorname{Spr} \mathcal{F})=F(\mathcal{F}),
$$

so that by Proposition IV.4.2 we have:

Proposition IV.7.2. The functor $F$ is t-exact, swaps tensor duality and Verdier duality, and is a faithful tensor functor $\mathbf{S p h}(\mathscr{G}) \rightarrow \mathbf{S p h}(\mathscr{T})$.

Proof. The $t$-exactness statement in Proposition IV.4.2 was reduced to this theorem, so we complete the proof here. By Proposition IV.6.13 it is enough to show that $F(\mathcal{J}(\lambda))$ is perverse for any $\lambda$. We will show that $F(\mathcal{J}(\lambda)) \in{ }^{p} \mathscr{D}(\mathscr{T})^{\leqslant 0}$; the dual claim is established by replacing $\mathcal{J}(\lambda)$ with its convolution dual.

$\mathcal{J}(\lambda)$ is supported on $\overline{\mathrm{Gr}}_{G}^{\lambda}$; for any coweight $\mu$, let $I^{\lambda, \mu}=\overline{\mathrm{Gr}}_{G}^{\lambda} \cap \mathrm{Gr}_{B}^{\mu}$; temporarily denote

$$
b: I^{\lambda, \mu} \rightarrow \overline{\mathrm{Gr}}_{G}^{\lambda}, \quad t: I^{\lambda, \mu} \rightarrow \mathrm{Gr}_{T}^{\mu} \cong \operatorname{Spec} \mathbb{C} ;
$$

then $F(\mathcal{J}(\lambda))^{\mu}=t_{!} b^{*} \mathcal{J}(\lambda)[\langle 2 \rho, \mu\rangle]$. By $[\mathrm{BBD} 82, \S 4.2 .4]$, the functor $t_{!}\left[\operatorname{dim} I^{\lambda, \mu}\right]$ is right $t$-exact. We claim that $b^{*}(\mathcal{J}(\lambda))\left[-\operatorname{codim} I^{\lambda, \mu}\right]$ is perverse. To express this, we make the additional notation

$$
\bar{b}: \overline{\mathrm{Gr}}_{B}^{\mu} \cap \overline{\mathrm{Gr}}_{G}^{\lambda} \rightarrow \overline{\mathrm{Gr}}_{G}^{\lambda}, \quad j: \mathrm{Gr}_{B}^{\mu} \rightarrow \overline{\mathrm{Gr}}_{B}^{\mu},
$$

so that $j$ is an open immersion and it suffices to show that $\bar{b}^{*}(\mathcal{J}(\lambda))\left[-\operatorname{codim} I^{\lambda, \mu}\right]$ is perverse. If $\lambda-\mu$ is not a sum of simple coroots then $I^{\lambda, \mu}$ is empty; otherwise, there is a chain of inequalities $\mu=\mu_{n}<\mu_{n-1}<\cdots<\lambda=\mu_{0}$ such that each $\mu_{k}-\mu_{k+1}$ is a simple coroot. The corresponding $\overline{\mathrm{Gr}}_{B}^{\mu_{k}}$ form a chain of successive Cartier divisors by Proposition II.5.2)(2), and thus so do their intersections with $\overline{\mathrm{Gr}}_{G}^{\lambda}$ (all nonempty and all intersecting $\mathrm{Gr}_{G}^{\lambda}$ as well). The claim then follows from Lemma IV.7.3, using Corollary IV.6.14

We have thus shown that $t_{!} b^{*}\left[\operatorname{dim} I^{\lambda, \mu}-\operatorname{codim} I^{\lambda, \mu}\right]$ is right $t$-exact on $\mathbf{S p h}(\mathscr{G})$. By Proposition IV.1.1, we have

$$
\operatorname{dim} I^{\lambda, \mu}=\langle\rho, \lambda+\mu\rangle, \quad \operatorname{codim} I^{\lambda, \mu}=\langle\rho, \lambda-\mu\rangle,
$$

whose difference is indeed $\langle 2 \rho, \mu\rangle$.

Lemma IV.7.3. Let $Y$ be a scheme. $Y=Y_{0} \supset Y_{1} \supset \cdots \supset Y_{n}$ be a sequence of successive Cartier divisors, and denote $i: Y_{n} \rightarrow Y$. Then $i^{*}[-n]$ is left $t$-exact. Furthermore, let $j_{U}: U \rightarrow Y$ be a smooth dense open subscheme and let $\mathcal{F}$ be lisse on $U$, perverse on $Y$, and suppose $\left.\left(j_{U}\right)_{!} \mathcal{F}\right|_{U} \cong \mathcal{F}$. Then if $U \cap Y_{i} \neq \varnothing$ for all $i<n$, then $i^{*}(\mathcal{F})[-n]$ is perverse. 
Proof. If we write $i_{k}$ for the inclusion of $Y_{k}$ in $Y_{k+1}$, then $i$ is the composition of all the $Y_{k}$ 's. By BBD82, Corollaire 4.1.10(ii)], each $i_{k}$ has cohomological amplitude $[-1,0]$, so their composition has amplitude $[-n, 0]$, as desired.

For the second claim, we also apply induction. Indeed, let $i_{n-1}: Y_{n-1} \rightarrow Y$, so since $U \cap Y_{n-1} \neq \varnothing$, we have $i_{n-1}^{*}\left(j_{U}\right) ! \cong\left(j_{U \cap Y_{n-1}}\right) ! i_{n-1}^{*}$. In particular, the natural map

$$
\left.\left(j_{U \cap Y_{n-1}}\right) !\left(i_{n-1}^{*} \mathcal{F}\right)\right|_{U \cap Y_{n-1}} \rightarrow i_{n-1}^{*} \mathcal{F}
$$

is again an isomorphism. By induction, it is an isomorphism of perverse sheaves after shifting by $n-1$. Thus, we may assume that $n=1$. But then the natural six-functors triangle

$$
\left(j_{U}\right) ! j_{U}^{*} \mathcal{F} \rightarrow \mathcal{F} \rightarrow i_{*} i^{*} \mathcal{F} \rightarrow
$$

shows that ${ }^{p} H^{0}\left(i^{*} \mathcal{F}\right)=0$, and we have already shown above that $i^{*} \mathcal{F}[-1]$ has cohomological amplitude [0,1], so it is perverse.

Tannakian duality. We will show that $\operatorname{Perv}(\mathscr{G})$ is equivalent to representations of the following group:

Definition IV.7.4. Let $G$ be a complex reductive group, $T \subset G$ a maximal torus with Weyl group $W$, and $Q \in Q\left(\Lambda_{T}, k^{*}\right)_{\mathbb{Z}}^{W}$ as in Theorem II.7.3. as above, let $\kappa$ be its symmetric bilinear form and $\Lambda_{Q}=\operatorname{ker}(\kappa)$. We define the root data of the twisted dual group $\check{G}_{Q}$ by the following description: it has a maximal torus $\check{T}_{Q}$ with $X^{*}\left(\check{T}_{Q}\right)=\Lambda_{Q}$, and its roots are all multiples of the coroots of $G$, and vice-versa, as follows. For every coroot $\check{\alpha}$, let $r=\operatorname{ord} Q(\check{\alpha})$ if this is finite. Then we have the corresponding root $\alpha_{Q}=r \check{\alpha}$ and coroot $\check{\alpha}_{Q}=\frac{1}{r} \alpha$.

Proposition IV.7.5. The root datum defined above is in fact a root datum.

Proof. Using the equation of Lemma II.7.1 with $\varepsilon_{\check{\alpha}}=1$, we see that $Q(\check{\alpha})^{\langle r \check{\alpha}, \lambda\rangle}=1$ for all $\lambda$ indeed implies $r \check{\alpha} \in \Lambda_{Q}$. Conversely, if $\lambda \in \Lambda_{Q}$, then, in particular, we have $\kappa(\check{\alpha}, \lambda)=1$, so $r \mid\langle\alpha, \lambda\rangle$ and $\frac{1}{r} \alpha \in\left(\Lambda_{Q}\right)^{\vee}$. Finally, note that if $Q(\check{\alpha})$ has infinite order, then no multiple of $\check{\alpha}$ is in $\Lambda_{Q}$.

It is obvious that $\left\langle\alpha_{Q}, \check{\alpha}_{Q}\right\rangle=\langle\alpha, \check{\alpha}\rangle=2$ and, in particular, $s_{\alpha_{Q}}=\left.s_{\check{\alpha}}\right|_{\Lambda_{Q}}$. Since $Q$ is $W$-invariant, we have $Q\left(s_{\check{\alpha}} \check{\beta}\right)=Q(\check{\beta})$ for any coroots $\check{\alpha}, \check{\beta}$, so the multiplier for a coroot is constant in $W$-orbits; it then follows that $s_{\alpha_{Q}}\left(\beta_{Q}\right)$ is another root in $\Lambda_{Q}$.

Since the codomain of $F$ is not in fact the category of vector spaces we must make some preparations before applying Tannakian duality. It follows from the combination of Proposition IV.6.16 and Proposition IV.5.1 that the values of $F$ are supported on $\mathrm{Gr}_{L} \breve{T}_{Q} \subset \mathrm{Gr}_{T}$ and, in fact, this restriction is sharp:

Lemma IV.7.6. Let $\lambda \in \Lambda_{Q}$ be any coweight which is dominant in $\Lambda$. Then $\mathscr{G}$ is equivariantly trivial on $\mathrm{Gr}_{G}^{\lambda}$ and, thus, we have an irreducible object $\mathcal{J}(\lambda) \in$ $\operatorname{Sph}(\mathscr{G})$.

Proof. Let $U \subset \operatorname{Gr}_{G}^{\lambda}$ be the intersection $\operatorname{Gr}_{G}^{\lambda} \cap \mathrm{Gr}_{B}^{\lambda}$, which by Proposition IV.1.1 is a dense open subset containing $t^{\lambda}=\mathrm{Gr}_{T}^{\lambda}$; since the Weyl group $W$ permutes the Borel subgroups, we also have open subsets $U^{w}$ for $w \in W$ contaning $t^{w(\lambda)}$, and these cover $\operatorname{Gr}_{G}^{\lambda}$. On each, $\mathscr{G}$ is trivial, since it descends to $\operatorname{Gr}_{T}$ from $\operatorname{Gr}_{B}$. Furthermore, clearly $B(\widehat{\mathcal{O}})$ acts on $U$, so $\left.\mathscr{G}\right|_{U}$ is trivially $B(\widehat{\mathcal{O}})$-equivariant. Likewise for the action of $w B w^{-1}(\widehat{\mathcal{O}})$ on $U^{w}$. 
Thus, the sheaf $\mathcal{F}_{U^{w}}=\underline{k}\left[-\operatorname{dim} \mathrm{Gr}_{G}^{\lambda}\right]$ on $U^{w}$ is perverse and $\left.\mathscr{G}\right|_{U^{w}}$-twisted, and so we have the $\mathscr{G}$-twisted perverse sheaf

$$
\mathcal{F}=\bigoplus_{W} j_{! *}^{w}\left(\mathcal{F}_{U^{w}}\right)
$$

where $j^{w}: U^{w} \rightarrow \operatorname{Gr}_{G}^{\lambda}$ is the open immersion. For any $b \in B(\widehat{\mathcal{O}})$ and any $w \in W$, we have $b U^{w} \cap U^{w} \neq \varnothing$ since $\operatorname{Gr}_{G}^{\lambda}$ is irreducible, so there is a natural $B(\widehat{\mathcal{O}})$-equivariance on $\mathcal{F}$ obtained by matching the summands of $b^{*} \mathcal{F}$ to those of $\mathcal{F}$ on these open intersections. Since clearly it is $W$-equivariant, by the Bruhat decomposition we have natural isomorphisms $g^{*} \mathcal{F} \cong \mathcal{F}$ for any $g \in G(\widehat{\mathcal{O}})$, giving $\mathcal{F}$ a $G(\widehat{\mathcal{O}})$-equivariance structure. By Lemma I.3.12, $\mathscr{G}$ is trivial on $\operatorname{Gr}_{G}^{\lambda}$.

Identifying perverse sheaves on this space with $\Lambda_{Q}$-graded, finite-dimensional vector spaces (the analogue of Proposition IV.5.2), the lemma shows that every weight of $\check{T}_{Q}$ can be obtained from an object of $\mathbf{S p h}(\mathscr{G})$. However, it is not the case that $F$ is a tensor functor to $\check{T}_{Q}$-representations.

Lemma IV.7.7. We have natural identifications

$$
\operatorname{Sph}(\mathscr{T}) \cong \mathscr{Z} \otimes \boldsymbol{R e p}\left(\check{T}_{Q}\right) \cong \boldsymbol{R e p}\left(\check{T}_{Q}\right)^{\prime},
$$

where $\mathscr{Z}=\left.\mathscr{Z}_{1}\right|_{x}$ (with $x \in X$ as before) is the fiber of the sf comultiplicative gerbe considered in Proposition IV.5.2, and thus a comultiplicative A-gerbe on $X$ (Lemma II.4.11), and $\operatorname{Rep}\left(\tilde{T}_{Q}\right)^{\prime}$ means altering the commutativity constraint in $\boldsymbol{\operatorname { R e p }}\left(\check{T}_{Q}\right)$ such that the product of two spaces of weights $\lambda$ and $\mu$ (with $\lambda, \mu \in \Lambda_{Q} \subset$ $\left.\Lambda_{T}\right)$ has the sign $Q(\lambda) Q(\mu)$.

Proof. We use Proposition II.4.13 to obtain the first equivalence; the equivalence of the first and third categories is the content of Proposition I.4.5. where the actual number was computed in Proposition II.4.3

We note that, in the lemma, the gerbe $\mathscr{Z}$ is trivial (since it is a gerbe on Spec $\mathbb{C}$ ), but not comultiplicatively trivial, in an instance of the principle suggested above Definition I.4.4. We also note that, according to Definition IV.7.4 and the number appearing in the lemma, $\mathscr{Z}$ is in fact a gerbe not for $\check{T}_{Q}(k)$ but for $Z\left(\check{G}_{Q}\right)(k)$, since $Q(\lambda)=0$ by definition if $\lambda$ is in the root lattice of $\check{G}_{Q}$.

We thus have $F: \operatorname{Sph}(\mathscr{G}) \rightarrow \mathscr{Z} \otimes \boldsymbol{R} \operatorname{ep}\left(\check{T}_{Q}\right)$, and we will want to move the $\mathscr{Z}$ from the right to the left. This is achieved by another small computation:

Lemma IV.7.8. Every object of $\mathbf{S p h}(\mathscr{G})$ admits an action of $Z\left(\check{G}_{Q}\right)$, giving $\mathbf{S p h}(\mathscr{G})$ an action of the "sheaf of categories" $\mathscr{H}^{1}\left(\operatorname{Spec} \mathbb{C}, Z\left(\breve{G}_{Q}\right)\right)$. With this structure, the functor

$$
F: \operatorname{Sph}(\mathscr{G}) \rightarrow \mathscr{Z} \otimes \operatorname{Rep}\left(\check{T}_{Q}\right)
$$

is $Z\left(\check{G}_{Q}\right)(k)$-equivariant.

Proof. Such an action of a torus is the same as a grading by its weight lattice. Every object of $\operatorname{Sph}(\mathscr{G})$ has a natural grading by $\pi_{1}(G)$, since the components of $\operatorname{Gr}_{G}$ are identified with this set; in fact, this grading is compatible with convolution, as follows from the fusion product (Proposition IV.3.4)(1)] . Since $\pi_{1}(G)$ is a quotient of $\Lambda_{T}$ and $\Lambda_{Q}$ is a subgroup, and since the root lattice of $\breve{G}_{Q}$ is contained in the coroot lattice of $G$, we obtain a grading by $X^{*}\left(Z\left(\check{G}_{Q}\right)\right)$. 
Ignoring the twisting by the trivial gerbe $\mathscr{Z}$, objects of the category $\operatorname{Rep}\left(\check{T}_{Q}\right)$ have a natural grading by $Z\left(\check{G}_{Q}\right)$, simply by grouping all the graded parts of each object whose degrees differ by sums of coroots of $G$. According to Proposition II.5.2) and Definition IV.7.1, this makes F compatible with the gradings, i.e., equivariant.

Multiplying $F$ by the inverse of $\mathscr{Z}$, we get a new tensor functor,

$$
F^{\prime}: \mathscr{Z}^{-1} \otimes \operatorname{Sph}(\mathscr{G}) \rightarrow \operatorname{Rep}\left(\check{T}_{Q}\right) .
$$

Note that since $\mathscr{Z}$ is trivial, the left-hand side merely has its commutativity constraint altered as well (by the same factor as above). By the Tannakian duality theorem of [DM], the functor $F^{\prime}$ induces an equivalence

$$
\mathscr{Z}^{-1} \otimes \mathbf{S p h}(\mathscr{G}) \cong \boldsymbol{R e p}(\check{G}),
$$

for some pro-algebraic $k$-group $\check{G}$ with a map from $\check{T}_{Q}$.

Properties of the dual group. Having shown that $\check{G}_{Q}$ is indeed the dual group for the twisted Satake equivalence, we proceed to identify it precisely.

Proposition IV.7.9. The group $\check{G}$ is algebraic, connected, and reductive.

Proof. We apply, respectively, the criteria of [DM, Prop. 2.20(b), 2.22, 2.23]. For algebraic, we choose any (finite) set of generators $\lambda_{i}$ of $\Lambda_{Q}$ and, by Lemma IV.7.6. construct the irreducible objects $\mathcal{J}\left(\lambda_{i}\right)$. Then by Corollary IV.6.10, every object of $\operatorname{Sph}(\mathscr{G})$ is a subquotient of some tensor polynomial in $\bigoplus \mathcal{J}\left(\lambda_{i}\right)$, so this is a tensor generator. For connected, we verify that no abelian subcategory generated by finitely many $\mathcal{J}(\lambda)$ 's is closed under convolution, which follows from Corollary IV.6.10 again since $\Lambda_{Q}$ is torsion-free. Finally, for reductive, we have Proposition IV.6.13.

The remainder of the section is devoted to the identification of $\check{G}$ as a reductive group. We will produce an isomorphism $\check{G}_{Q} \rightarrow \check{G}$ according to the following strategy: we will identify a Borel subgroup of $\breve{G}$ and show that its corresponding dominant weights are identified with those coweights in $\Lambda_{Q}$ which are dominant for $G$, and hence $\check{G}_{Q}$. Then we will pick out the simple roots of $\check{G}$ and apply the following lemma:

Lemma IV.7.10. Let $H$ and $H^{\prime}$ be reductive groups with maximal tori $U, U^{\prime}$ that are isomorphic; suppose further that $C$ and $C^{\prime}$ are Borel subgroups containing these tori, the choice of which identifies the dominant weights in $X^{*}(U)$ with those in $X^{*}\left(U^{\prime}\right)$ under this isomorphism. Suppose that for every simple root $\alpha$ of $H$, with corresponding Levi factor $L$ (whose only simple root is $\alpha$ ), there is a commutative diagram

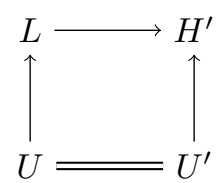

Then there is a unique isomorphism $H \rightarrow H^{\prime}$ extending these maps.

To prove this, we need an even smaller lemma on algebraic groups, which proves itself. 
Lemma IV.7.11. Let $K, L$ be reductive groups with maximal tori $S, U$. Let $f: L \rightarrow$ $K$ be an algebraic group homomorphism such that $\left.f\right|_{S}$ is an isomorphism of $S$ with $U$. Let $\alpha$ be a root of $L$ and in the Lie algebra $\mathfrak{l}$, let $v$ be a weight vector for the adjoint action of $L$, with weight $\alpha \in X^{*}(U)=X^{*}(S)$. Then $d f(v)$ is a weight vector with weight $\alpha$ for the adjoint action of $K$ on $\mathfrak{k}$, so $\alpha$ is a root of $K$.

Proof of Lemma IV.7.10. We apply Lemma IV.7.11 to $H^{\prime}$ and $L$, concluding that $\alpha$ is a root of $H^{\prime}$ for any simple root $\alpha$ of $H$. The collection of all the $\alpha$ determine the set of dominant weights in $X^{*}(U)=X^{*}\left(U^{\prime}\right)$ as those weights $\lambda$ such that $\langle\lambda, \check{\alpha}\rangle \geq 0$ for every $\alpha$ which is a simple root of $H$. But that means that $\{\alpha\}$ determines the Weyl chamber of weights corresponding to $C^{\prime}$, and therefore to the basis determined by $C^{\prime}$. Since $\{\alpha\}$ is a basis for the weight lattice, it is in fact the basis for the root sytem corresponding to $C^{\prime}$.

Thus, $H$ and $H^{\prime}$ have the same simple roots; we claim that they have the same coroots as well. Indeed, each Levi $L$ corresponding to $\alpha$ has $\check{\alpha}$ as a simple coroot, and the map $L \rightarrow H^{\prime}$ sends $\check{\alpha}$ to some coroot of $H^{\prime}$ (which is of course equal to $\check{\alpha}$, since the tori in $L$ and $H^{\prime}$ are identified). By definition of the simple reflections, $\check{\alpha}$ is negated by $s_{\alpha}$, which means that it is a multiple of the coroot $\check{\beta}$ of $H^{\prime}$ dual to $\alpha$, and since $\langle\alpha, \check{\alpha}\rangle=2=\langle\alpha, \breve{\beta}\rangle$, that multiple must be 1 .

Therefore there is an isomorphism between the root data of $H$ and $H^{\prime}$, so there is a unique group isomorphism, identifying the Levi factors, that induces it.

We have already argued that $\check{T}_{Q}$ admits a map into $\check{G}$ because $F^{\prime}$ takes values in $\Lambda_{Q^{-}}$graded vector spaces. By Lemma IV.7.6 in fact, the image of $F^{\prime}$ generates $\operatorname{Rep}\left(\check{T}_{Q}\right)$, so by [DM, Prop. 2.21(b)] this map is a closed immersion. To show it is maximal, we consider the Borel subgroup.

By the Plücker relations, a Borel subgroup in $\check{G}$ is specified by giving, for every irreducible $V^{\lambda} \in \operatorname{Rep}(\check{G})$, a line $\ell^{\lambda}$ in it, such that for any other irreducible $V^{\mu}$, the line $\ell^{\lambda} \otimes \ell^{\mu} \in V^{\lambda} \otimes V^{\mu}$ agrees with the line $\ell^{\lambda+\mu}$ lying in the (unique) summand $V^{\lambda+\mu}$ inside the tensor product. We take $\ell^{\lambda}$ to be the weight space $F^{\prime}(\mathcal{J}(\lambda))^{\lambda}$ and apply Corollary IV.6.10 and the fact that $F^{\prime}$ is a tensor functor to obtain this for $\operatorname{Sph}(\mathscr{G})$. We will denote this Borel subgroup by $\check{B}_{Q}$; by definition, the dominant weights of $\check{G}$ with respect to $\check{B}_{Q}$ are exactly the dominant $\lambda$ which lie in $\Lambda_{Q}$.

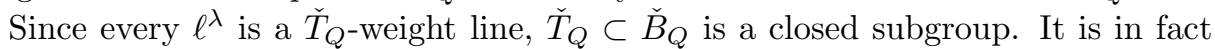
a maximal torus, since the $\lambda$-weight line has multiplicity one. Thus, the first two criteria of Lemma IV.7.10 are satisfied, and we need only produce the maps of Levi factors.

Semisimple rank 1. We now appeal to the "parabolic fiber functors" $F_{n}^{P}$ defined in Definition IV.4.4, following the apparently original appearance of this technique in [BD, 5.3.27-31]. Let $\alpha$ be any simple root of $G$ and let $P$ be the parabolic subgroup corresponding to $\alpha$, with Levi quotient $L$, a reductive group of semisimple rank 1 having the same maximal torus $T$. We denote by $\mathscr{L}$ the gerbe on $\operatorname{Gr}_{L}$ induced by $\mathscr{G}$. As for the plain fiber functor $F$, by Proposition IV.4.5 it induces a faithful, exact tensor functor

$$
F^{P}: \operatorname{Sph}(\mathscr{G}) \rightarrow \operatorname{Sph}(\mathscr{L})
$$

factoring the fiber functor. By the Tannakian formalism we have a group homomorphism $\check{L} \rightarrow \check{G}$. It remains only to prove that $\check{L} \cong \check{L}_{Q}$ in order to apply 
Lemma IV.7.10, so we now assume that $G$ has semisimple rank 1. The following lemma allows us to focus on the semisimple groups of rank 1:

Lemma IV.7.12. The root lattice of $\breve{G}$ in $\Lambda_{Q}$ must be contained in the coroot lattice of $G$, so that when $G$ has semisimple rank 1 , the roots of $\breve{G}$ are multiples of the coroots of $G$.

Proof. A highest-weight representation $V^{\lambda}$ of a reductive group has its highest weight $\lambda$ in the root lattice of that group if and only if its weight space $V^{\lambda}(0) \neq 0$. By Definition IV.7.1, this holds in $\mathbf{S p h}(\mathscr{G})$ only if $\mathrm{Gr}_{B}^{0}=\mathrm{Gr}_{T}^{0}$ intersects the support of $\mathcal{J}(\lambda)$, so $\operatorname{Gr}_{G}^{\lambda}$ must be in the connected component of $\operatorname{Gr}_{G}$ which contains $\mathrm{Gr}_{T}^{0}$, so $\lambda$ is in the coroot lattice.

To pass from a general group $G$ of semisimple rank 1 to a semisimple one, we replace $G$ by its (semisimple) derived subgroup $G^{\prime}=[G, G]$, whose coweight lattice is the rational coroot lattice $\Lambda_{T, r}^{\mathbb{Q}}$ as defined in Lemma II.7.2. Suppose we write, similarly to that lemma but using Theorem II.7.3.

$$
\mathscr{G}_{n}=\mathscr{K}_{n} \otimes \mathscr{M}_{n},
$$

where $\mathscr{K}_{n}$ is of "Killing type", in that its quadratic form is a product of Killing forms and its associated multiplicative gerbe is trivial, and $\mathscr{M}_{n}$ is of "multiplicative type", in that its quadratic form descends to $\Lambda_{T} / \Lambda_{T, r}^{\mathbb{Q}}$. Therefore, we have that

$$
Q_{\mathscr{G}}=Q_{\mathscr{K}} Q_{\mathscr{M}}, \quad \kappa_{\mathscr{G}}=\kappa_{\mathscr{K}} \kappa_{\mathscr{M}},
$$

and since $Q_{\mathscr{M}}\left(\Lambda_{T, r}^{\mathbb{Q}}\right)=1$ and $\kappa_{\mathscr{M}}\left(\Lambda_{T, r}^{\mathbb{Q}}, \Lambda_{T}\right)=1$, we have that $\mathscr{M}$ is $G(\widehat{\mathcal{O}})$ equivariantly trivial on $\mathrm{Gr}_{G^{\prime}}$, and then

$$
\operatorname{Sph}\left(\mathscr{G} \mid \operatorname{Gr}_{G^{\prime}}\right) \cong \operatorname{Sph}\left(\mathscr{K} \mid \operatorname{Gr}_{G^{\prime}}\right),
$$

so that the latter category is independent of whether we consider $G(\widehat{\mathcal{O}})$-equivariant sheaves or $G^{\prime}(\widehat{\mathcal{O}})$-equivariant sheaves (since if $\langle\alpha, \lambda\rangle=1$ for all roots $\alpha$, we also have $\kappa_{\mathscr{K}}(\alpha, \lambda)=1$; thus, the presence of the central coweights does not affect $\left.\Lambda_{Q_{\mathscr{K}}}\right)$, and finally, that the induced map

$$
\check{G} \rightarrow\left(G^{\prime}\right)^{\vee}
$$

which is a surjection by [DM, Proposition 2.21(a)], induces an isomorphism on root lattices. Since $\Lambda_{T, r}^{\mathbb{Q}}$ contains (by construction) all fractional multiples of the coroots of $G$, the multipliers appearing in Definition IV.7.4 are the same for $G$ as for $G^{\prime}$, so we may replace $G$ with $G^{\prime}$.

Therefore, we may assume that $G=\mathrm{SL}_{2}, \mathrm{PGL}_{2}$ is one of the two semisimple groups of rank 1 . We will denote by $\mathscr{G}_{\mathrm{SL}_{2}}$ or $\mathscr{G}_{\mathrm{PGL}_{2}}$ the sf gerbe on their grassmannians. Then $\Lambda_{T} \cong \mathbb{Z}$ for each, the nontrivial element of $W$ acts as negation, and the quadratic form $Q$ is necessarily of the form $Q(n)=q_{0}^{n^{2}}$ for some $q_{0} \in k^{*}$. Clearly, the natural map $\mathrm{SL}_{2} \rightarrow \mathrm{PGL}_{2}$ induces an inclusion $\Lambda_{\mathrm{SL}_{2}}=2 \Lambda_{\mathrm{PGL}_{2}}$, and if we identify the latter with $\mathbb{Z}$, sends $Q$ (for $\mathrm{PGL}_{2}$ ) to the quadratic form on $\Lambda_{\mathrm{SL}_{2}}$, $n \mapsto q_{0}^{4 n^{2}}$. The following result is a quick computation:

Lemma IV.7.13. Suppose $G=\mathrm{PGL}_{2}, \widetilde{G}=\mathrm{SL}_{2}$ and identify the lattice of the former with $\mathbb{Z}$, that of the latter with $2 \mathbb{Z}$; let $q_{0}=Q(1)$ have order $r_{0} \in \mathbb{N} \cup\{\infty\}$. Then $\Lambda_{Q, G}=\Lambda_{Q, \widetilde{G}}$ is zero if $r_{0}$ is infinite, and otherwise:

- If $r_{0}$ is odd, then $\Lambda_{Q, \widetilde{G}}=r_{0} \mathbb{Z}=2 \Lambda_{Q, G}$.

- If $\operatorname{ord}_{2} r_{0}=1$, then $\Lambda_{Q, \widetilde{G}}=r_{0} \mathbb{Z}=2 \Lambda_{Q, G}$. 
- If $\operatorname{ord}_{2} r_{0}=2$, then $\Lambda_{Q, G}=\left(r_{0} / 2\right) \mathbb{Z}=\Lambda_{Q, \widetilde{G}}$.

- If $\operatorname{ord}_{2} r_{0} \geq 3$, then $\Lambda_{Q, G}=\left(r_{0} / 2\right) \mathbb{Z}=2 \Lambda_{Q, \widetilde{G}}$.

Now we are prepared to compute $\check{G}$ when $G$ has semisimple rank 1 .

Proposition IV.7.14. Let $G$ be a semisimple group of rank 1 with simple coroot $\check{\alpha}$; let $r$ be the order of $q=Q(\check{\alpha}) \in k^{*}$. Then the positive root of $\check{G}$ is r $\check{\alpha}$, if $r$ is finite, and otherwise $\breve{G}$ is trivial.

Proof. We consider the map $\mathrm{SL}_{2} \rightarrow \mathrm{PGL}_{2}$, which induces a map $\mathrm{Gr}_{\mathrm{SL}_{2}} \rightarrow \mathrm{Gr}_{\mathrm{PGL}_{2}}$ identifying the former with the connected component of the latter which contains $\mathrm{Gr}_{T}^{0}$. Let $\mathscr{G}_{\mathrm{PGL}_{2}}$ be the fiber of an sf gerbe on the latter, with quadratic form $Q$, and let $\mathscr{G}_{\mathrm{SL}_{2}}$ be its restriction to this component, inducing a form on the weight lattice of $\mathrm{SL}_{2}$. Since we have $\mathrm{SL}_{2} \rightarrow \mathrm{PGL}_{2}$, the natural restriction makes $\mathscr{G}_{\mathrm{PGL}_{2}}$ an $\mathrm{SL}_{2}(\widehat{\mathcal{O}})$-equivariant gerbe on the common component and we have $\mathbf{S p h}\left(\mathscr{G}_{\mathrm{PGL}_{2}}\right)^{\circ} \subset$ $\operatorname{Sph}\left(\mathscr{G}_{\mathrm{SL}_{2}}\right)$, the circle denoting objects supported on $\mathrm{Gr}_{\mathrm{SL}_{2}}$.

If $r$ is infinite, then $\kappa$ is nondegenerate on $\Lambda_{T}$ (for either $\mathrm{SL}_{2}$ or $\mathrm{PGL}_{2}$ ), so $\Lambda_{Q}=0$ and therefore, since $F$ is faithful, $\mathbf{S p h}\left(\mathscr{G}_{\mathrm{SL}_{2}}\right)=\mathbf{S p h}\left(\mathscr{G}_{\mathrm{PGL}_{2}}\right) \cong \operatorname{Vect}_{k}$ (generated by the trivial sheaf supported on $\left.\mathrm{Gr}_{G}^{0}\right)$. Thus, $\breve{G}=1$ in either case.

If not, then we have a few possibilities. First, note that when $\Lambda_{Q} \neq 0$, the dual group $\mathrm{SL}_{2}$ is not a torus, since in fact any $\mathcal{J}(\lambda)$ with $\lambda \neq 0$ is irreducible but has at least two nonzero weight spaces $F(\mathcal{J}(\lambda))^{ \pm \lambda}$.

Now suppose $r_{0}$ is odd; then, by Lemma IV.7.13, we have $\Lambda_{Q, \mathrm{SL}_{2}} \subset \Lambda_{Q, \mathrm{PGL}_{2}}$, so by faithfulness of $F$, we also have $\mathbf{S p h}\left(\mathscr{G}_{\mathrm{SL}_{2}}\right) \subset \mathbf{S p h}\left(\mathscr{G}_{\mathrm{PGL}_{2}}\right)$, so we have a surjection $\breve{S L}_{2} \rightarrow \mathrm{PGL}_{2}$ of which the first, as noted, is not a torus so neither is the second. They are thus both semisimple of rank 1; since the map from one to the other sends roots to roots by Lemma IV.7.11 the computation in Lemma IV.7.13 shows that the common simple root must be $2 r_{0}=r_{0} \check{\alpha}$. Since $q_{0}$ has odd order, $q=q_{0}^{4}$ has the same order $r=r_{0}$.

If $\operatorname{ord}_{2} r_{0}=1$, then we have the same inclusion of lattices, and therefore the common root is $r_{0}=\left(r_{0} / 2\right) \check{\alpha}$. However, note that since $q=q_{0}^{4}=\left(q_{0}^{2}\right)^{2}$, we actually have $r=r_{0} / 2$, so again the root is $r \check{\alpha}$.

If $\operatorname{ord}_{2} r_{0} \geq 3$, then the inclusion of lattices is reversed. Thus, $\operatorname{Sph}\left(\mathscr{G}_{\mathrm{PGL}_{2}}\right) \subset$ $\operatorname{Sph}\left(\mathscr{G}_{\mathrm{SL}_{2}}\right)$ and we have $\Lambda_{Q, \mathrm{PGL}_{2}}=2 \Lambda_{Q, \mathrm{SL}_{2}}$, so that by the computations of the lemma the common root is necessarily $r_{0} / 2=\left(r_{0} / 4\right) \check{\alpha}$. Since $q=q_{0}^{4}$ and $q_{0}$ has order a multiple of 4 , in fact $r=r_{0} / 4$, so again the root is $r \check{\alpha}$.

Now suppose $\operatorname{ord}_{2} r_{0}=2$. Then by the computations of Lemma IV.7.13, we have $\Lambda_{Q, \mathrm{SL}_{2}}=\Lambda_{Q, \mathrm{PGL}_{2}}$, so $\mathrm{SL}_{2} \cong \mathrm{PGL} \breve{L}_{2}$ but we cannot extract the root from this comparison. The quadratic form on $\Lambda_{\mathrm{SL}_{2}}$ is defined by $q=q_{0}^{4}$, which has odd order $r=r_{0} / 4$, and is therefore the fourth power of some other $q_{1}$ with the same order (namely, $q_{1}=q^{a}$, where $4 a+r b=1$ ). We replace $\mathscr{G}_{\mathrm{PGL}_{2}}$ by the fiber of the sf gerbe defined by the quadratic form corresponding to $q_{1}$; then we are back in the case where $r_{0}$ is odd, and so $\mathrm{SL}_{2}=\mathrm{PGL}_{2}$, so the root is the smallest element of $\Lambda_{Q, \mathrm{SL}_{2}}=\Lambda_{Q, \mathrm{PGL}_{2}}$, namely $r_{0} / 2=2\left(r_{0} / 4\right)=r \check{\alpha}$ once again.

In all of the above computations, we have shown that when $\mathscr{G}_{\mathrm{SL}_{2}}$ is inherited from $\mathrm{PGL}_{2}$, then the proposition holds. Suppose we are given only an sf $k^{*}$-gerbe $\mathscr{G}_{\mathrm{SL}_{2}, n}$ with its quadratic form, determined by the number $q$. Let $\phi: k \rightarrow l$ be an inclusion of fields in which $q=q_{0}^{4}$ with $q_{0} \in l$, and let $\mathscr{H}_{\mathrm{SL}_{2}, n}={ }^{2} \phi\left(\mathscr{G}_{\mathrm{SL}_{2}}\right)$ be the induced sf $l^{*}$-gerbe. Let $X$ be a small disk and, by Theorem II.7.3. let $\mathscr{H}_{\mathrm{PGL}_{2}, n}$ be the sf gerbe corresponding to the $l^{*}$-valued quadratic form defined 
by $q_{0}$. Then both gerbes are entirely determined by their quadratic form, so in particular $\mathscr{H}_{\mathrm{SL}_{2}}$ really is determined by $\mathscr{H}_{\mathrm{PGL}_{2}}$ by the map $\mathrm{Gr}_{\mathrm{SL}_{2}} \rightarrow \mathrm{Gr}_{\mathrm{PGL}_{2}}$. Then by the above computations, the split $l^{*}$-group $\check{G}_{l}$ obtained from $\mathrm{SL}_{2}$ from $\mathscr{H}_{\mathrm{SL}_{2}}$ has the correct root data. However, clearly we have $\mathbf{S p h}\left(\mathscr{H}_{\mathrm{SL}_{2}}\right)=\mathbf{S p h}\left(\mathscr{G}_{\mathrm{SL}_{2}}\right) \otimes_{k} l$, so $\check{G}_{l} \cong \check{\mathrm{SL}}_{2} \otimes_{\text {Spec } k}$ Spec $l$ and so $\check{\mathrm{SL}}_{2}$ has the correct root system as well. This completes the proof.

Going back to (IV.11), we can restate our result in the form originally intended:

Theorem IV.7.15. The fiber functor $F$ induces an equivalence

$$
\operatorname{Sph}(\mathscr{G}) \cong \mathscr{Z} \otimes \boldsymbol{\operatorname { R e p }}\left(\check{G}_{Q}\right) \cong \boldsymbol{\operatorname { R e p }}\left(\check{G}_{Q}\right)^{\prime},
$$

where the prime denotes modifying the commutativity constraint of the tensor product of spaces of weight $\lambda$ and $\mu$ by $Q(\lambda) Q(\mu)$.

\section{IV.8. Relative twisted Satake}

All that has already come is sufficient preparation to easily deduce nonlocal versions of the results of the previous section.

Proposition IV.8.1. Let $\mathscr{G}_{n}$ be an sf $k^{*}$-gerbe on $\mathrm{Gr}_{G, X^{n}}$, and let $\mathscr{Z}_{n}$ be the sf comultiplicative $\operatorname{Fact}\left(\check{T}_{Q}(k)\right)_{n}$-gerbe on $X^{n}$ which has already appeared in Proposition IV.5.2, Then $\mathscr{Z}_{n}$ is in fact defined over $\operatorname{Fact}\left(Z\left(\check{G}_{Q}\right)(k)\right)_{n}$.

Proof. Let $\mathscr{T}_{n}$ be the sf gerbe on $\mathrm{Gr}_{T, X^{n}}$ corresponding to $\mathscr{G}_{n}$; by Proposition II.4.9, we must show that $\mathscr{T}_{n}$ is sf-trivial on the sf-scheme within $\operatorname{Gr}_{T, X^{n}}$ consisting of components $\operatorname{Gr}_{T, X^{n}}^{\lambda_{1}, \ldots, \lambda_{n}} \cong X^{n}$ in which all the $\lambda_{i}$ are in the root lattice of $\check{G}_{Q}$. By Proposition II.3.6, it suffices to show that the quadratic form $Q$ (which is a homomorphism on $\Lambda_{Q}$ since its associated bilinear form is trivial) and the associated commutative multiplicative gerbe $\mathscr{M}$ are trivialized on the roots of $\check{G}_{Q}$. This is tautologically true for $Q$ by Definition IV.7.4. Similarly, $\mathscr{M}$, as a $\Lambda_{T}$-multiplicative gerbe, is trivialized on the coroot lattice $\Lambda_{T, r}$ (that is, it is multiplicative for $\pi_{1}(G)=$ $\left.\Lambda_{T} / \Lambda_{T, r}\right)$, and therefore also on the root lattice of $\check{G}_{Q}$, again by definition.

Theorem IV.8.2. With notation as in Proposition IV.8.1, $F_{n}$ induces an equivalence of rigid tensor abelian categories

$$
\operatorname{Sph}^{U L A}\left(\mathscr{G}_{n}\right) \cong \mathscr{Z}_{n} \otimes \operatorname{Rep}^{U L A}\left(\check{G}_{Q}^{n}\right),
$$

where the representations are taken in local systems (i.e., ULA perverse sheaves) on $X^{n}$.

Proof. We have by definition

$$
F_{n}: \mathbf{S p h}^{\mathrm{ULA}}\left(\mathscr{G}_{n}\right) \rightarrow \mathbf{S p h}^{\mathrm{ULA}}\left(\mathscr{T}_{n}\right),
$$

where $\mathscr{T}_{n}$ is, as usual, the sf gerbe on $\operatorname{Gr}_{T, X^{n}}$ corresponding to $\mathscr{G}_{n}$. By Proposition IV.5.2 and Proposition IV.5.5, we have

$$
\operatorname{Sph}^{\mathrm{ULA}}\left(\mathscr{T}_{n}\right) \cong \mathscr{Z}_{n} \otimes \operatorname{FRep}_{n}^{\mathrm{ULA}}\left(\check{T}_{Q}\right)
$$

as tensor categories, the latter is identified with factorizable representations of $\check{T}_{Q}$ in locally constant sheaves, so we collapse the notation and write

$$
F_{n}: \operatorname{Sph}^{\mathrm{ULA}}\left(\mathscr{G}_{n}\right) \rightarrow \mathscr{Z}_{n} \otimes \mathbf{F R e p}_{n}^{\mathrm{ULA}}\left(\check{T}_{Q}\right) .
$$


The first step is to prove the analogue of Lemma IV.7.8 for $\mathbf{S p h}^{\mathrm{ULA}}\left(\mathscr{G}_{n}\right)$ and $F_{n}$ so that we can move $\mathscr{Z}_{n}$ from one side to the other. The proof is the same: over any open set $U \subset X^{n}$ having $m$ distinct coordinates, the connected components of $\left.\operatorname{Gr}_{G, X^{n}}\right|_{U}$ are indexed by $\pi_{1}(G)^{n}$ and thus objects of $\mathbf{S p h}{ }^{\mathrm{ULA}}\left(\left.\mathscr{G}_{n}\right|_{U}\right)$ are graded by $\pi_{1}(G)^{m}$, and thus degenerately by $Z\left(\check{G}_{Q}\right)^{m}$. Since this indexing of components corresponds to the inclusion of the various $\mathrm{Gr}_{T, X^{n}}^{\lambda_{1}, \ldots, \lambda_{n}}$, Proposition II.1.2 shows that the corresponding actions of $Z\left(\check{G}_{Q}\right)^{m}$ assemble to an action of $\operatorname{Fact}\left(Z\left(\check{G}_{Q}\right)\right)_{n}$. As in Lemma IV.7.8, one sees that $F_{n}$ is equivariant for this action, so we may consider the functor

$$
F_{n}^{\prime}: \mathscr{Z}_{n}^{-1} \otimes \operatorname{Sph}^{\mathrm{ULA}}\left(\mathscr{G}_{n}\right) \rightarrow \mathbf{F R e p}^{\mathrm{ULA}}\left(\check{T}_{Q}^{n}\right) .
$$

By Proposition IV.4.2, $F_{n}$ is faithful and exact and, by Corollary IV.4.3, a tensor functor, so these are true of $F_{n}^{\prime}$ as well; the last one, which is not tautological, follows from Proposition I.4.2 using the sf multiplicativity of $\mathscr{Z}_{n}$ (Proposition II.4.9).

By Proposition IV.6.16 and Theorem IV.7.15, in a small neighborhood $D$ of any $\vec{x} \in X^{n}$ with distinct coordinates, $F_{n}^{\prime}$ induces an equivalence

$$
\left.\mathscr{Z}_{n}^{-1}\right|_{D} \otimes \operatorname{Sph}\left(\left.\mathscr{G}_{n}\right|_{D}\right) \cong \operatorname{Rep}^{\mathrm{ULA}}\left(\check{G}_{Q}^{n}\right) .
$$

These equivalences are compatible with restriction to smaller neighborhoods, so, by Lemma IV.2.4, induce a $\check{G}_{Q}^{n}$-action on every $\mathcal{F} \in \mathscr{Z}_{n}^{-1} \otimes \operatorname{Sph}^{\mathrm{ULA}}(\mathscr{G})$.

If $U \subset X^{n}$ is the open set with distinct coordinates, then

$$
F_{n}:\left.\mathbf{S p h}^{\mathrm{ULA}}\left(\left.\mathscr{G}_{n}\right|_{U}\right) \rightarrow \mathscr{Z}_{n}\right|_{U} \otimes \mathbf{F R e p}_{n}^{\mathrm{ULA}}\left(\check{T}_{Q}\right)
$$

factors through $\left.\mathscr{Z}_{n}\right|_{U} \otimes \operatorname{Rep}_{n}^{\mathrm{ULA}}\left(\check{G}_{Q}^{n}\right)_{U}$, where

$$
\operatorname{Rep}_{n}^{\mathrm{ULA}}\left(\check{G}_{Q}^{n}\right)_{U} \cong \mathbf{F R e p}_{n}^{\mathrm{ULA}}\left(\check{G}_{Q}\right)_{U}
$$

Since restriction of ULA sheaves to $U$ is inverted by minimal extension by Proposition IV.2.8, in fact, $F_{n}$ factors through globally as well.

We now state the main result of this work.

Theorem IV.8.3. With notation as in Proposition IV.8.1, the fiber functor $F_{n}$ induces an equivalence

$$
\operatorname{Sph}\left(\mathscr{G}_{n}\right) \cong \mathscr{Z}_{n} \otimes \operatorname{FRep}_{n}\left(\check{G}_{Q}\right)
$$

compatibly with their respective outer convolutions.

Proof. The combination of the definition of $F_{n}$ and Proposition IV.5.2 gives a map from $\mathbf{S p h}\left(\mathscr{G}_{n}\right)$ to $\mathscr{Z}_{n} \otimes \mathbf{F R e p}_{n}\left(\check{T}_{Q}\right)$, and Proposition IV.4.2 shows that it respects convolution. Lemma IV.2.14 may be applied since $F_{n}$ is horizontal and preserves ULA sheaves, so we successively construct a functor $\operatorname{Sph}\left(\mathscr{G}_{n}\right) \rightarrow \mathscr{Z}_{n} \otimes \mathbf{F R e p}\left(\check{G}_{Q}\right)$, an isomorphism verifying that it factors $F_{n}$ through the forgetful functor to $\mathscr{Z}_{n} \otimes$ $\operatorname{FRep}\left(\check{T}_{Q}\right)$, and an inverse functor making it an equvalence.

\section{Part V. Connections}

The main result of this work, Theorem IV.8.3, is (at present) the endpoint of a long chain of development. Thus, we close with a few remarks on how it fits into the larger scheme of things. 


\section{V.1. Relation to the Result of FinkelberG-Lysenko}

The main result of Finkelberg-Lysenko [FL10] can be stated in our language as follows, assuming as usual that $G$ is a complex reductive algebraic group and $T$ a maximal torus (however, that paper considers more general algebraically closed fields of any characteristic).

Theorem. (Finkelberg-Lysenko, [FL10, Theorem 1]) Let d be fixed as described in loc. cit. following their Proposition 1, let $\breve{h}$ be the dual Coxeter number of $G$, and choose a positive integer $N$. Denote by $E^{c}$ a $(2 \check{h} / d)$ th root of the determinant line bundle $\operatorname{det}(\mathrm{adj})$ on the ordinary affine grassmannian $\mathrm{Gr}_{G}$ and let $q$ be an $N$ th root of unity. Write $\mathscr{G}=\left(E^{c}\right)^{\log q}$; then we have

$$
\operatorname{Sph}(\mathscr{G}) \cong \operatorname{Rep}(\check{G})
$$

where $\check{G}$ is the reductive algebraic group with the following root datum. Fix the notation

$$
\begin{array}{ll}
\iota: \Lambda_{T} \rightarrow \Lambda^{T}, & \iota(\lambda)=\frac{1}{2 \check{h}} \sum_{\alpha}\langle\alpha, \lambda\rangle \alpha, \\
(\bullet, \bullet): \Lambda_{T} \otimes \Lambda_{T} \rightarrow \mathbb{Z}, & (\lambda, \mu)=\langle\iota(\lambda), \mu\rangle
\end{array}
$$

(the sum running over all roots $\alpha$ ). Then we have for $\check{G}$ :

(1) its weight lattice consists of those $\lambda \in \Lambda_{T}$ such that $d \iota(\lambda) \in N \Lambda^{T}$;

(2) for each coroot $\check{\alpha}$ of $G$, the multiple $r \alpha$ is a root, where $r$ is the denominator of the fraction $d(\alpha, \alpha) / 2 N$ written in lowest terms.

The coweights of $\breve{G}$ are dual to its weights, and the coroots are the multiples $\alpha / r$.

It should be noted that the statement of this theorem is incorrect in one particular: it lacks the modified commutativity constraint given in Theorem IV.7.15. This error can be traced to the beginning of [FL10, §4.2], where Tannakian duality is prematurely invoked without fully computing the tensor category structure of the left-hand side. However, the dual group itself agrees with ours:

Proposition V.1.1. Let $\mathscr{G}$ have quadratic form $Q$; then $\check{G}=\check{G}_{Q}$.

Proof. Note that, since $\left(E^{c}\right)^{2 \breve{h} / d}=\operatorname{det}(\operatorname{adj})$, we can write just as well that $\mathscr{G}=$ $\operatorname{det}(\operatorname{adj})^{d / 2 \breve{h} N}$. By Proposition II.6.8, the associated quadratic form $Q$ is the exponential of a primitive $1^{d / 2 \mathscr{h} N}$ by the integer-valued form

$$
\frac{1}{2} \sum_{\alpha}\langle\alpha, \lambda\rangle^{2}=\check{h}\langle\iota(\lambda), \lambda\rangle=\check{h}(\lambda, \lambda),
$$

the sum running over all roots $\alpha$. Its associated bilinear form $\kappa$ is likewise the exponential of this primitive root of unity by the integer-valued form

$$
\sum_{\alpha}\langle\alpha, \lambda\rangle\langle\alpha, \mu\rangle=2 \check{h}\langle\iota(\lambda), \mu\rangle=2 \check{h}(\lambda, \mu) .
$$

Thus, $\lambda \in \operatorname{ker}(\kappa)=\Lambda^{\breve{T}_{Q}}$ if and only if $2 \check{h} N / d$ divides the value in (V.2) for all $\mu \in \Lambda_{T}$, or if $d \iota(\lambda) \in N \Lambda^{T}$, which is the condition of point (1) In addition, we choose the roots in this lattice to be multiples $r \check{\alpha}$ of the coroots $\check{\alpha}$, where $r=\operatorname{ord} Q(\check{\alpha})$. According to (V.1), $r$ is the least integer such that $2 \check{h} r(\check{\alpha}, \check{\alpha})$ is a multiple of $2 \check{h} N / d$; i.e., the denominator of $d(\check{\alpha}, \check{\alpha}) / 2 N$, as given in point $(2)$. 


\section{V.2. Relation to Lusztig's quantum Groups}

In Lus93, §2.2.5], Lusztig transforms a root datum $\left(X_{*}, X^{*}, \Phi_{*}, \Phi^{*}\right)$ by means of a specified positive integer $l$ and a choice of associated "Cartan datum", defined as follows:

Definition. (Lusztig Lus93, §1.1.1]) A Cartan datum is a finite set $I$ together with a symmetric bilinear pairing,

$$
\bullet \bullet: \mathbb{Z}[I] \otimes \mathbb{Z}[I] \rightarrow \mathbb{Z},
$$

such that $i \cdot i$ is even and positive for all $i \in I$ and $2(i \cdot j) /(j \cdot j)$ is a nonpositive integer for all distinct $i, j \in I$. A root datum of type $I$ is a quadruple $\left(X_{*}, X^{*}, \phi_{*}, \phi^{*}\right)$ consisting of a pair of dual finitely-generated free abelian groups $X_{*}, X^{*}$ and the embeddings,

$$
\phi_{*}: I \rightarrow X_{*}, \quad \phi^{*}: I \rightarrow X^{*},
$$

such that we have $\left\langle\phi^{*}(i), \phi_{*}(j)\right\rangle=2(i \cdot j) /(j \cdot j)$ for all $i, j$.

However, since we begin with a root datum $\left(X_{*}, X^{*}, \Phi_{*}, \Phi^{*}\right)$ defined in the usual way, we prefer to consider this definition differently:

Definition V.2.1. Let $D=\left(X_{*}, X^{*}, \Phi_{*}, \Phi^{*}\right)$ be a root datum (that is, a pair of dual free abelian groups of finite type together with roots and coroots in them satisfying the usual axioms). To specify a Cartan datum attached to $D$ is to give:

(1) A base for $D$; that is, a set of positive roots and their corresponding coroots. We denote by $I$ the abstract set of positive roots, $\phi_{*}, \phi^{*}$ its inclusions into $X_{*}$ and $X^{*}$.

(2) A $W$-invariant integer-valued quadratic form $f$ on $\mathbb{Z}[I]$ (which is identified via the $\phi$ 's with both the root and coroot lattices) taking positive values on $I$.

It is not hard to see that these are actually the same definition:

Lemma V.2.2. Definition V.2.1 agrees with Lusztig's definition.

Proof. Let $b$ be the bilinear form associated with $f$ (that is, $f(i+j)=f(i)+$ $b(i, j)+f(j))$ and define $i \cdot j=b(i, j)$. Then $i \cdot i=b(i, i)=2 f(i)$ is even (a fortiori) and positive (a priori). That $2(i \cdot j) /(j \cdot j)$ is nonpositive will follow from

$$
f(j)\left\langle\phi_{*}(i), \phi^{*}(j)\right\rangle=b(i, j),
$$

since the pairing between two simple roots is always nonpositive. In fact, this is just a restatement of $W$-invariance of $f$ :

$$
\begin{aligned}
f(j) & =f\left(s_{i}(j)\right)=f\left(j-\left\langle\phi^{*}(i), \phi_{*}(j)\right\rangle i\right) \\
& =f(j)-\left\langle\phi^{*}(i), \phi_{*}(j)\right\rangle b(j, i)+\left\langle\phi^{*}(i), \phi_{*}(j)\right\rangle^{2} f(i),
\end{aligned}
$$

so trivial algebra gives the desired equation when $\left\langle\phi^{*}(i), \phi_{*}(j)\right\rangle \neq 0$. Otherwise, it follows from Lemma II.7.1, since $\mathbb{Z}$ is torsion-free.

Note that in Definition V.2.1, we have $f(i)=(i \cdot i) / 2$. It is apparent that, root datum in hand, the only substantial choice in the definition of an associated Cartan datum is that of the quadratic form $f$; we will continue to refer to the whole Cartan datum just using the letter $f$. Following Lusztig, we make the following definition of another root datum: 
Definition. Fix a root datum $D$ and associated Cartan datum $f$ as in Definition V.2.1. Let $l$ be any positive integer and for each $i$, let $l_{i}=l / \operatorname{gcd}(l, f(i))$. Then the dual root datum constructed from $f$ and $l$ is $D_{f, l}=\left(Y_{*}, Y^{*}, \Psi_{*}, \Psi^{*}\right)$, where

(1) $Y^{*}=\left\{\lambda \in X_{*} \mid \forall i \in I,\left\langle\phi^{*}(i), \lambda\right\rangle \in l_{i} \mathbb{Z}\right\}$.

(2) $\psi^{*}(i)=l_{i} \phi_{*}(i)$.

Likewise, $Y_{*}$ is the dual of $Y_{*}$ and $\psi_{*}(i)=\phi^{*}(i) / l_{i}$.

We will show that this construction almost coincides with a special case of that given in Definition IV.7.4. To this end, let $q$ be a primitive $l$ th root of unity in $k$ (an algebraically closed field of characteristic zero, which can in fact be any ring for this construction) and define a $k^{*}$-valued quadratic form on $\mathbb{Z}[I]$ (equivalently, the coroot lattice in $X_{*}$ )

$$
Q(\lambda)=q^{f(\lambda)}
$$

Since $Q$ is not defined on all of $X_{*}$ it is not possible to apply our construction literally. However, we can perform one much like it, as follows. We extend the bilinear form $b$ defined by $f$ to $\mathbb{Z}[I] \otimes X_{*}$ by taking

$$
b(i, \lambda)=f(i)\left\langle\phi^{*}(i), \lambda\right\rangle
$$

and extending by linearity. Note that $b\left(i, \phi_{*}(j)\right)=f(i)\left\langle\phi^{*}(i), \phi_{*}(j)\right\rangle=b(i, j)$ as previously defined/proven in Lemma V.2.2. If we take

$$
\kappa(i, \lambda)=q^{b(i, \lambda)},
$$

a $k^{*}$-valued bilinear form, then its restriction to $\mathbb{Z}[I] \otimes \phi_{*}(\mathbb{Z}[I])$ is the bilinear form defined by $Q$. Furthermore, its right kernel in $X_{*}$ is the set of all $\lambda$ such that, for each $i \in I$, we have

$$
f(i)\left\langle\phi^{*}(i), \lambda\right\rangle=b(i, \lambda) \in l \mathbb{Z},
$$

or equivalently, $\left\langle\phi^{*}(i), \lambda\right\rangle \in l_{i} \mathbb{Z}$, as required by (1) Likewise, since $l_{i}$ is the least positive integer such that $l_{i} f(i) \in l \mathbb{Z}$, it is the order of $Q(i)$ and therefore taking the roots in this subspace to be $l_{i} \phi_{*}(i)$ agrees with (2). In summary:

Proposition V.2.3. Let $G$ be a reductive group with root datum $D, \Lambda_{T}$ its coweight lattice and $\Lambda_{T, r}$ the coroot lattice. For any $k^{*}$-valued bilinear form $\kappa$ on $\Lambda_{T, r} \times \Lambda_{T}$ and quadratic form $Q$ defining its restriction to $\Lambda_{T, r} \times \Lambda_{T, r}$, each coming from $W$ invariant forms valued in $\mathbb{Z}$ as in Lemma II.7.2, we define dual root data $D_{Q, \kappa}$ as in Definition IV.7.4 but taking $\Lambda^{\check{T}_{Q}}$ to be the right kernel of $\kappa$. Then if $Q=q^{f}$ with $f$ an integer-valued quadratic form and $q$ an lth root of unity, we have $D_{Q, \kappa}=$ $D_{f, l}$.

The generality of this construction is incomparable to that of Definition IV.7.4 though if $Q$ extends to all of $\Lambda$ and the resulting bilinear form has the same kernel as $\kappa$, the two give the same result. We do not know of a geometric interpretation of such quadratic forms, though Lemma II.7.2 is relevant for bilinear forms defined on $\Lambda_{T, r} \times \Lambda_{T}$.

In addition, although it is possible (as in the above proposition) to weaken the hypotheses of Definition IV.7.4 without losing the ability to perform the construction of the dual group, the full generality of a quadratic form defined on the whole of $\Lambda$ is necessary in order to obtain the modified commutativity constraint exhibited in Theorem IV.7.15. This modification thus does not occur in Lusztig's work. 


\section{V.3. Alteration of the commutativity constraint}

On the subject of the commutativity constraint, it should be noted that we have introduced two modifications: one in Theorem IV.7.15 and depending on the gerbe $\mathscr{G}_{n}$, and the other in Corollary IV.4.3 and depending only on $\operatorname{Gr}_{G, X}$. In fact, by judicious twisting, one can reduce the latter to the former, thus tuning a jarring note in both our exposition (see Section IV.4) and that of [MV07. The appropriate gerbe is in fact quite significant:

Lemma V.3.1. Let adj be the adjoint representation of $G$, whose weights are exactly the roots of $G$ (and zero). Then $\operatorname{det}(\operatorname{adj})^{\log (-1)}$ has quadratic form

$$
Q(\lambda)=(-1)^{\langle 2 \rho, \lambda\rangle}
$$

and trivial bilinear form. Furthermore, this gerbe is trivial (as a gerbe, but not as an sf gerbe).

Proof. Since that quadratic form is valued in 2-torsion elements, the bilinear form is automatically trivial, so it suffices to establish just the equation. Then we have, denoting roots by $\alpha$,

$$
\log _{-1} Q(\lambda)=\frac{1}{2} \sum_{\alpha}\langle\alpha, \lambda\rangle^{2}=\sum_{\alpha>0}\langle\alpha, \lambda\rangle^{2} .
$$

Since by definition $2 \rho=\sum_{\alpha>0} \alpha$, we see that

$$
\langle 2 \rho, \lambda\rangle^{2} \equiv \sum_{\alpha>0}\langle\alpha, \lambda\rangle^{2} \bmod 2
$$

and of course we have $\langle 2 \rho, \lambda\rangle^{2} \equiv\langle 2 \rho, \lambda\rangle \bmod 2$. To show that $\operatorname{det}(\operatorname{adj})^{\log (-1)}$ is trivial on $\operatorname{Gr}_{T, X^{n}}$, it suffices by Proposition II.6.8 to show that $\mathcal{T}_{X}^{\log (-1)}$ is trivial on $X$. Choose a theta-characteristic on $X$; i.e., a square root $\mathcal{T}_{X}^{1 / 2}=\mathcal{T}_{X}^{-1 / 2}$ of the canonical sheaf. Then the total space of $\mathcal{T}_{X}^{1 / 2}$ is a two-fold covering of that of $\mathcal{T}_{X}$, and its sheaf of sections is thus a fortiori a $\mathcal{L}_{-1}$-twisted local system on $\mathcal{T}_{X}$, trivializing $\mathcal{T}_{X}^{\log (-1)}$ according to Proposition I.5.3.

We note that the dual group $\check{G}_{Q}$ obtained from the above quadratic form is isomorphic to ${ }^{L} G$, since $\langle 2 \rho, \alpha\rangle=2$ for any coroot $\alpha$. Therefore, if we let $\mathscr{G}=$ $\operatorname{det}(\operatorname{adj})^{\log (-1)}$ on $\mathrm{Gr}_{G}$, we can replace the usual statement of the geometric Satake equivalence with the one that $\mathbf{S p h}(\mathscr{G}) \cong \boldsymbol{\operatorname { R e p }}\left({ }^{L} G\right)$, where the left-hand side has its natural commutativity constraint, i.e., not modified, and is equivalent as a monoidal category to $\mathbf{S p h}_{G}$. This suggests that the Satake equivalence naturally concerns $\mathscr{G}$-twisted perverse sheaves even in the "untwisted" case. From the proof of the lemma, we also see that this twisting is precisely the "critical twisting" considered everywhere in $[\mathrm{BD}$.

\section{V.4. Relation to The QUANTUM LANGLANDS CORRESPONDENCE}

It is possible to relate sf gerbes for $G$ to those for ${ }^{L} G$, the Langlands dual, under some hypotheses. Suppose that we are given a nondegenerate $\mathbb{C}$-valued (additive) $W$-invariant bilinear form $b: \Lambda_{T} \times \Lambda_{T} \rightarrow \mathbb{C}$ (note that this automatically comes from a $\mathbb{Z}$-valued form as in Lemma II.7.2, since $\mathbb{C}$ is torsion-free); this induces a $\mathbb{C}^{*}$ valued (multiplicative) form $\kappa(\lambda, \mu)=\exp (2 \pi i b(\lambda, \mu))$ and quadratic form $Q(\lambda)=$ $\exp (\pi i b(\lambda, \lambda))$. Then $b$ is equivalent to an isomorphism $f: \mathbb{C} \otimes \Lambda_{T} \rightarrow \mathbb{C} \otimes \Lambda^{T}$; 
$f^{-1}$ is equivalent to a nondegenerate bilinear form ${ }^{L} b$ on $\mathbb{C} \otimes \Lambda^{T}=\mathbb{C} \otimes \Lambda_{L_{T}}$. Let ${ }^{L} Q(\lambda)=\exp \left(\pi i^{L} b(\lambda, \lambda)\right)$.

Proposition V.4.1. The dual group of Definition IV.7.4 associated with the pair $(G, Q)$ is isomorphic to that of the pair $\left({ }^{L} G,{ }^{L} Q\right)$, where ${ }^{L} G$ is, as usual, the Langlands dual of $G$.

Proof. We must show that there is an isomorphism of their weight lattices identifying their roots, and that the dual isomorphism identifies the coroots.

For $\check{G}_{Q}$, we have $\Lambda^{\breve{T}_{Q}}=\operatorname{ker}(\kappa)=f^{-1}\left(\Lambda^{T}\right) \cap \Lambda_{T}$; likewise, for $\left({ }^{L} G\right)_{L}^{\vee}$, we have

$$
\Lambda^{\left({ }^{L} T\right)_{L_{Q}}^{\vee}}=\Lambda^{T} \cap f\left(\Lambda_{T}\right) .
$$

Thus, we choose the isomorphism

$$
f: \Lambda^{\check{T}_{Q}} \rightarrow \Lambda^{\left({ }^{L} T\right)_{L}^{\vee}}
$$

Now let $\alpha \in \Lambda^{T}$ be a root of $G$ and $\check{\alpha}$ the corresponding coroot; then the $W$ invariance of $b$ implies the $W$-equivariance of $f$ and, therefore, that $f(\check{\alpha}) \in \mathbb{C} \alpha$; likewise $f^{-1}(\alpha) \in \mathbb{C} \check{\alpha}$. It follows that $Q(\check{\alpha})$ has finite order if and only if $f(\check{\alpha}) \in \mathbb{Q} \alpha$, and likewise ${ }^{L} Q(\alpha)$ has finite order if and only if $f^{-1}(\alpha) \in \mathbb{Q} \check{\alpha}$, which is equivalent. More precisely, if $f(\check{\alpha})=(k / n) \alpha$ with $\operatorname{gcd}(k, n)=1$, then the corresponding root in $\Lambda^{\breve{T}_{Q}}$ is $n \check{\alpha}$ by definition. We may write this as

$$
f(n \check{\alpha})=k \alpha,
$$

which is also equivalent to $f^{-1}(\alpha)=(n / k) \check{\alpha}$, where $\operatorname{gcd}(n, k)=1$ and thus, ${ }^{L} Q(\alpha)$ has order $k$; so the corresponding root of $\left({ }^{L} G\right)_{L_{Q}}$ is $k \alpha$, and the preceding equation shows that $f$ sends the root of $\check{G}_{Q}$ to that of $\left({ }^{L} G\right)_{L_{Q}}^{\vee}$.

Finally, since the new coroots are respectively $\alpha / n$ and $\check{\alpha} / k$, the map on coweights induced by $f$ (that is, $f^{-1}$ ) also sends roots to roots, and thus $f$ is an isomorphism of root data.

Given this setup, let $\mathscr{G}_{n}$ be the sf $\mathbb{C}^{*}$-gerbe corresponding to the quadratic form ${ }^{L} Q$, and let ${ }^{L} \mathscr{G}_{n}$ be the one corresponding to ${ }^{L} Q$, with their multiplicativegerbe parts trivial. Denote by $\mathscr{Z}_{n}$ and ${ }^{L} \mathscr{Z}_{n}$ the gerbes for $\operatorname{Fact}\left(Z\left(\check{G}_{Q}\right)(k)\right)_{n} \cong$ $\operatorname{Fact}\left(Z\left(\left({ }^{L} G\right)_{L_{Q}}^{\vee}\right)(k)\right)_{n}$ appearing in Theorem IV.8.3.

Lemma V.4.2. We have $\mathscr{Z}_{n} \cong{ }^{L} \mathscr{Z}_{n}$.

Proof. Let $\mathscr{T}_{n}$ and ${ }^{L} \mathscr{T}_{n}$ be the corresponding gerbes on $\mathrm{Gr}_{T}$ and $\mathrm{Gr}_{L} T$. According to Proposition II.4.9, each of the $\mathscr{Z}$ gerbes corresponds to the restrictions of $\mathscr{T}_{n}$ and $L \mathscr{T}_{n}$ to $\operatorname{Gr}_{L} \check{T}_{Q}$ and $\operatorname{Gr}_{L}(L T)_{L_{Q}}^{\vee}$, respectively, as multiplicative factorizable gerbes. The multiplicative structure is uniquely determined by the factorizable structure according to Proposition II.4.3, and this, in turn, is uniquely determined by the restrictions of the quadratic forms $Q$ and $\left({ }^{L} Q\right)$ to $\Lambda_{L_{T_{Q}}}$ and $\Lambda_{L}\left({ }^{L} T\right)_{L}^{\vee}{ }_{Q}$ (respectively), by Proposition II.3.6. These are identified via $f$ as in the proof of Proposition V.4.1.

Therefore, applying Theorem IV.8.3 to both $\operatorname{Sph}\left(\mathscr{G}_{n}\right)$ and $\operatorname{Sph}\left({ }^{L} \mathscr{G}_{n}\right)$ (the former a category of sheaves on $\mathrm{Gr}_{G, X^{n}}$ and the latter of sheaves on $\operatorname{Gr}_{L} G, X^{n}$, we find that they are equivalent. This is a local version of the quantum Langlands correspondence. 


\section{REFERENCES}

[BL95] A. Beauville and Y. Laszlo, Un lemme de descente, C. R. Acad. Sci. Paris Sér. I Math. 320 (1995), no. 3, 335-340 (French, with English and French summaries). MR.1320381 (96a:14049)

[BBD82] A. Beilinson, J. Bernstein, and P. Deligne, Faisceaux pervers, Analysis and topology on singular spaces, I (Luminy, 1981), Astérisque, vol. 100, Soc. Math. France, Paris, 1982, pp. 5-171 (French). MR751966 (86g:32015)

[BD] A. Beilinson and V. Drinfeld, Quantization of Hitchin's integrable system and Hecke eigensheaves, available at http://www.math.uchicago.edu/ mitya/langlands/ hitchin/BD-hitchin.pdf.

[Bra03] T. Braden, Hyperbolic localization of intersection cohomology, Transform. Groups 8 (2003), no. 3, 209-216. MR1996415 (2004f:14037)

[BG02] A. Braverman and D. Gaitsgory, Geometric Eisenstein series, Invent. Math. 150 (2002), no. 2, 287-384. MR 1933587 (2003k:11109)

[BFGM02] A. Braverman, M. Finkelberg, D. Gaitsgory, and I. Mirković, Intersection cohomology of Drinfeld's compactifications, Selecta Math. (N.S.) 8 (2002), no. 3, 381-418. MR 1931170 (2003h:14060)

[Dri01] V. Drinfeld, On a conjecture of Kashiwara, Math. Res. Lett. 8 (2001), no. 5-6, 713-728.

[DM] P. Deligne and J. Milne, Tannakian categories, Hodge Cycles, Motives, and Shimura Varieties, Lecture Notes in Mathematics, vol. 900, Springer, 1981.

$\left[\mathrm{FGI}^{+} 05\right]$ B. Fantechi, L. Göttsche, L. Illusie, S. Kleiman, N. Nitsure, and A. Vistoli, Fundamental algebraic geometry, Mathematical Surveys and Monographs, vol. 123, Amer. Math. Soc., 2005. MR2222646 (2007f:14001)

[FL10] M. Finkelberg and S. Lysenko, Twisted geometric Satake equivalence, J. Inst. Math. Jussieu 9 (2010), no. 4, 719-739. MR2684259 (2011i:22020)

[FGV01] E. Frenkel, D. Gaitsgory, and K. Vilonen, Whittaker patterns in the geometry of moduli spaces of bundles on curves, Ann. of Math. (2) 153 (2001), no. 3, 699-748. MR.1836286 (2002e:11156)

[Gai07] D. Gaitsgory, On de Jong's conjecture, Israel J. Math. 157 (2007), 155-191.

[Gin95] V. Ginzburg, Perverse sheaves on a Loop group and Langlands' duality (1995), available at http://arxiv.org/abs/alg-geom/9511007.

[Gir71] J. Giraud, Cohomologie non abélienne, Die Grundlehren der mathematischen Wissenschaften, Band 179, Springer-Verlag, 1971 (French). MR0344253 (49:8992)

[Lus93] G. Lusztig, Introduction to quantum groups, Progress in Mathematics, vol. 110, Birkhäuser Boston Inc., Boston, MA, 1993. MR1227098 (94m:17016)

[Lus83] _ Singularities, character formulas, and a q-analog of weight multiplicities, Analysis and topology on singular spaces, II, III (Luminy, 1981), Astérisque, vol. 101, Soc. Math. France, Paris, 1983. MR737932 (85m:17005)

[MV07] I. Mirković and K. Vilonen, Geometric Langlands duality and representations of algebraic groups over commutative rings, Ann. of Math. (2) 166 (2007), no. 1, 95-143. MR:2342692 (2008m:22027)

[Rei10] R. Reich, Notes on Beilinson's "How to glue perverse sheaves", Journal of Singularities 1 (2010), 94-115, available at http://arxiv.org/abs/1002.1686v4. MR2671769 (2011j:14039)

UCla Mathematics Department, 520 Portola Plaza, los Angeles, California 90095

E-mail address: ryanr@math.ucla.edu

Current address: Department of Mathematics, University of Michigan, 2074 East Hall, 530 Church Street, Ann Arbor, Michigan 48109-1043 